\title{
Learning from the Artist: Theory and Practice of Example-Based Character Deformation
}

by

John Lewis

\author{
A thesis \\ submitted to the Victoria University of Wellington \\ in fulfilment of the \\ requirements for the degree of \\ Doctor of Philosophy \\ in Computer Science.
}

Victoria University of Wellington

2016 



\begin{abstract}
Movie and game production is very laborious, frequently involving hundreds of person-years for a single project. At present this work is difficult to fully automate, since it involves subjective and artistic judgments.

Broadly speaking, in this thesis we explore an approach that works with the artist, accelerating their work without attempting to replace them. More specifically, we describe an "example-based" approach, in which artists provide examples of the desired shapes of the character, and the results gradually improve as more examples are given. Since a character's skin shape deforms as the pose or expression changes, or particular problem will be termed character deformation.

The overall goal of this thesis is to contribute a complete investigation and development of an example-based approach to character deformation. A central observation guiding this research is that character animation can be formulated as a high-dimensional problem, rather than the two- or three-dimensional viewpoint that is commonly adopted in computer graphics. A second observation guiding our inquiry is that statistical learning concepts are relevant. We show that example-based character animation algorithms can be informed, developed, and improved using these observations.

This thesis provides definitive surveys of example-based facial and body skin deformation.

This thesis analyzes the two leading families of example-based character deformation algorithms from the point of view of statistical regression. In doing so we show that a wide variety of existing tools in machine learning are applicable to our problem. We also identify several techniques
\end{abstract}


that are not suitable due to the nature of the training data, and the highdimensional nature of this regression problem. We evaluate the design decisions underlying these example-based algorithms, thus providing the groundwork for a "best practice" choice of specific algorithms.

This thesis develops several new algorithms for accelerating examplebased facial animation. The first algorithm allows unspecified degrees of freedom to be automatically determined based on the style of previous, completed animations. A second algorithm allows rapid editing and control of the process of transferring motion capture of a human actor to a computer graphics character.

The thesis identifies and develops several unpublished relations between the underlying mathematical techniques.

Lastly, the thesis provides novel tutorial derivations of several mathematical concepts, using only the linear algebra tools that are likely to be familiar to experts in computer graphics.

Portions of the research in this thesis have been published in eight papers, with two appearing in premier forums in the field. 


\section{Acknowledgments}

Thanks to my great friends and colleagues Alex Ma, Jaewoo Seo, Yeongho Seol, Ken Anjyo, Ayumi Kimura, James Jacobs, Pondy, Dale Carnegie, and the Victoria graphics group. Particular thanks goes to my supervisors Dr. Taehyun Rhee and Prof. Mengjie Zhang for their generous guidance and support during my study.

I am enormously grateful to people who have taken time to personally explain some math to me, including Ken Anjyo, Jean-Marie Aubry, Hiroyuki Ochiai, Masato Wakayama, Geoffrey Irving, Eitan Grinspun, Luca Fascione, Miguel Sepulveda, David Balduzzi, Stephen Marsland, Georgy Gimel'farb. Similar thanks to the CaSP and Festival of Doubt groups at Victoria. Development of the ideas in this thesis benefited from various discussions with Lance Williams over the years. Marcus Frean and Alex Ma shared insights about high-dimensional phenomena and Gaussian processes. Lance Williams, Fred Parke, Craig Reynolds, and Thad Beier provided information on the early history of blendshapes. David Bibby and Shona DeSain applied special powers to get this effort started.

I would especially like to thank (again) Taehyun Rhee and Ken Anjyo. They have been both friends and the best collaborators one could ask for.

Models and animation data were obtained from Hiroki Itokazu, Bret St. Clair, Tatsuo Yotsukura, Ng Jiahao, Nickson Fong and Egg Story Creative Productions, Kyle Labad, and Raymond Chan. Motion capture data was obtained from mocap.cs.cmu.edu, created with funding from NSF EIA-0196217. 


\section{List of Publications}

1. J.P. Lewis, Ken Anjyo, Taehyun Rhee, Superresolution from Principal Component Models by RKHS Sampling, in Mathematical Progress in Expressive Image Synthesis II, Springer, 2015.

2. Ozan Cetinaslan, Vernica Orvalho, John Lewis, Sketch-Based Controllers for Blendshape Facial Animation, Eurographics 2015.

3. J.P. Lewis, Ken Anjyo, Taehyun Rhee, Mengjie Zhang, Frederic Pighin, Zhigang Deng, Survey and Theory of Blendshape Facial Animation Computer Graphics Forum, submitted for publication, 2015.

4. J.P. Lewis, Ken Anjyo, Taehyun Rhee, Mengjie Zhang, Frederic Pighin, Zhigang Deng, Practice and Theory of Blendshape Facial Models, Eurographics 2014.

5. J.P. Lewis, Zhenyao Mo, Ken Anjyo, Taehyun Rhee, Probable and improbable faces, Mathematical Progress in Expressive Image Synthesis, Springer, 2014.

6. J.P. Lewis, Taehyun Rhee, Mengjie Zhang, Principal Component Analysis and Laplacian Splines: Steps toward a Unified Model, in M. Wakayama et al., The Impact of Applications on Mathematics, Springer, 2014.

7. Yeongho Seol, J.P. Lewis, Jaewoo Seo, Byungkuk Choi, Ken Anjyo, Junyong Noh, Spacetime Expression Cloning for Blendshapes ACM Transactions on Graphics, April 2012. 
8. Ken Anjyo, Hideki Todo, J.P. Lewis A Practical Approach to Direct Manipulation Blendshapes, Journal of Graphics Tools, Volume 16, Issue 3, 2012.

The author also contributed to the following publications that are not related to the thesis:

1. John P. Lewis, Realism and Texture: Benchmark Problems for Natural Computation, Unconventional Computation and Natural Computation 2015.

2. Agata Migalska and J.P. Lewis, An Information Theoretic Approach to Reflectional Symmetry Detection, IVCNZ 2015.

3. Evgeny Patrikeev and John Lewis, Texture-aware Edit Propagation using Nonparametric Regression, IVCNZ 2015.

4. Cristian S. Calude, Alasdair Coull, J.P. Lewis, Can we Solve the Pipeline Problem, ACM Digipro 2014.

5. Richard Roberts, Tim Jones, J.P. Lewis, Synthesis of Incidental Detail as Composable Components in a Functional Language, IVCNZ 2013.

6. Wan-chun Ma, Marco Barbati, J.P. Lewis, A Facial Composite Editor for Blendshape Characters, ACM Digipro 2012.

7. Yeongho Seol, Jaewoo Seo, Paul Hyunjin Kim, J.P. Lewis, Junyong Noh, Weighted Pose Space Editing for Facial Animation, The Visual Computer, 28(3) 2012.

8. Cristian S. Calude, J.P. Lewis, Is there a Universal Image Generator? Applied Mathematics and Computation 218, 16, 2012.

Alphabetical author order was used on two publications that were coauthored with a mathematician, following the convention in mathematics. 


\section{Contents}

1 Introduction 1

1.1 Problem Statement and Motivations . . . . . . . . . . . 1

1.2 Research Goals . . . . . . . . . . . . . . . . . . . . 4 4

1.3 Contributions . . . . . . . . . . . . . . . . 4

1.4 Organization of the Thesis $\ldots \ldots \ldots \ldots \ldots$

2 Background 9

2.1 Deformation for Character Animation . . . . . . . . . 9

2.1 .1 Generic algorithms . . . . . . . . . . . . . . . 10

2.1 .2 Parametric approaches . . . . . . . . . . . . . 11

2.1 .3 Physically-based approaches . . . . . . . . . . 11

2.1 .4 Skinning . . . . . . . . . . . . . . . . 13

2.2 Chapter Summary . . . . . . . . . . . . . . . . . 17

I Linear Models 19

3 Linear Facial Models 21

3.1 Terminology . . . . . . . . . . . . . . . . . . . . 25

3.2 History $\ldots \ldots \ldots \ldots \ldots \ldots \ldots$

3.3 Algebra and Algorithms . . . . . . . . . . . . . . 29

$3.3 .1 \quad$ Delta blendshape formulation . . . . . . . . . 29 29

3.3 .2 Intermediate shapes $\ldots \ldots \ldots \ldots$. . . . . . . . 31

3.3 .3 Combination blendshapes . . . . . . . . . . . . 33

vii 
3.3 .4 Hybrid rigs . . . . . . . . . . . . . 35

3.4 Constructing Blendshapes . . . . . . . . . . . . 35

3.4 .1 Model transfer . . . . . . . . . . . . . . 36

3.4 .2 Discovery of blendshapes . . . . . . . . . . 37

3.4 .3 Blendshape refinement. . . . . . . . . . . . . . 39

3.4 .4 Detail enhancement . . . . . . . . . . . . . 4 40

3.4 .5 Interpolating to make new models . . . . . . . . . . 40

3.4 .6 Compressing blendshapes . . . . . . . . . . . . . . . . . . . . . . . . .

3.4 .7 Outlook ....................... 42

3.5 Animation and Interaction Techniques . . . . . . . . . . 443

3.5 .1 Keyframe animation . . . . . . . . . . . . 43

3.5 .2 Performance-driven animation . . . . . . . . . 4 43

3.5 .3 Expression cloning . . . . . . . . . . . . . . 45

3.5 .4 Stabilization . . . . . . . . . . . . . . 50

3.5 .5 Partially-automated animation . . . . . . . . . . 51

3.5 .6 Direct manipulation . . . . . . . . . . . . . 52

3.5 .7 Further interaction techniques . . . . . . . . 56

3.5 .8 Outlook ..................... 5 57

$3.6 \quad$ Facial Animation as an Interpolation Problem. . . . . . . . 5 57

3.6.1 Blendshapes as a high dimensional interpolation problem ................... 57

3.6 .2 Linear interpolation . . . . . . . . . . . 5 57

3.6 .3 Scattered interpolation . . . . . . . . . . . . . 58

3.6.4 Blendshapes as a tangent space . . . . . . . . . . 59

3.6 .5 Outlook . . . . . . . . . . . . . . . 60 60

3.7 The Blendshape Parameterization . . . . . . . . . . . . . 61 61

3.7.1 Lack of orthogonality . . . . . . . . . . . . . 61

3.7 .2 Blendshape models are not unique . . . . . . . . . 62

3.7.3 Equivalence of whole-face and delta blendshape formulations .................62

3.7 .4 Global versus local control . . . . . . . . . . . 64 
3.7.5 Convex combination of shapes . . . . . . . . . 65

3.7 .6 Semantic parameterization . . . . . . . . . . 67

3.7 .7 PCA is not interpretable . . . . . . . . . . . . . 69

3.7.8 Conversion between blendshape and PCA representations ................. 73

3.7.9 Probability of a blendshape expression . . . . . . 73

3.7.10 Outlook .................... 76

3.8 Generalizations and Future Directions . . . . . . . . . . . 76

3.8 .1 Outlook ..................... 78

3.9 Chapter Summary . . . . . . . . . . . . . . . . 78

4 Accelerating Facial Editing with Statistical Priors 81

4.1 Introduction . . . . . . . . . . . . . . . . . . 83

4.2 Method ....................... 86

$4.2 .1 \quad$ Direct Manipulation of a Given Model . . . . . . . . . 86

4.2 .2 Pinning. . . . . . . . . . . . . . . . . 88

$4.2 .3 \quad$ Haphazard movements . . . . . . . . . . . . . . 89

4.2 .4 Implementation . . . . . . . . . . . . . . . . 91

4.2.5 Relative power of direct manipulation versus parametric control . . . . . . . . . . . . . . . . . 91

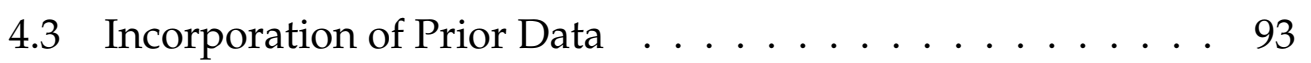

$4.3 .1 \quad$ An extension to higher order statistics . . . . . . . 96

4.4 Results .......................... 98

4.5 Chapter Summary . . . . . . . . . . . . . . . . . 99

5 Space-Time Expression Cloning 103

5.1 Introduction . . . . . . . . . . . . . . 103

5.2 Formulation as a Poisson Equation . . . . . . . . . . . . 104

5.3 Formulation for Blendshapes . . . . . . . . . . . . . . 106

5.4 Temporal Derivative as a Matrix Expression . . . . . . . . . . 107

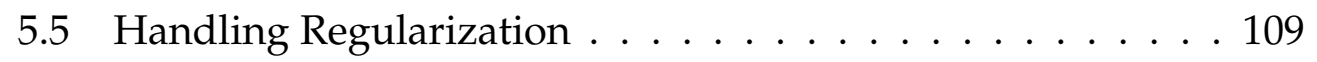

5.6 Chapter Summary . . . . . . . . . . . . . . . . . . . 110 
6 Exploring the Character of Face Space 113

6.1 Introduction . . . . . . . . . . . . . . . . . . . . . 114

6.2 Linear models . . . . . . . . . . . . . . . . . . . . . . . 114

6.3 High-dimensional Phenomena $\ldots . . . \ldots \ldots$

6.4 The High-Dimensional Gaussian Prior . . . . . . . . . . . . 118

6.5 Example Computation: Interpolating in Face Space . . . . . 123

6.6 Chapter Summary . . . . . . . . . . . . . . . . . . 124

II Nonlinear Models 125

7 The Example-Based Approach to Creature Skinning 127

7.1 Introduction . . . . . . . . . . . . . . . . . . . . . . 129

7.2 EBS Algorthms . . . . . . . . . . . . . . . . . . . . . 132

7.2 .1 Pose Space Deformation . . . . . . . . . . . . . . 132

$7.2 .2 \quad$ Shape by Example . . . . . . . . . . . . . . . . . 136

7.2 .3 Weighted Pose Space Deformation . . . . . . . . . . 137

7.2.4 Context-Aware Skeletal Shape Deformation . . . . . 138

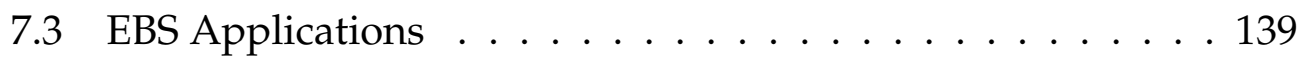

$7.3 .1 \quad$ Skeleton-Driven Deformation . . . . . . . . . . . . 139

7.3 .2 Secondary Animation . . . . . . . . . . . . . . . 139

7.3.3 Using PSD to "Reparameterize" an Existing Rig ... 139

7.3 .4 Facial Animation . . . . . . . . . . . . . . . . . . 140

7.3 .5 Wrinkles and Clothing . . . . . . . . . . . . . . 143

7.3 .6 Retargeting . . . . . . . . . . . . . . 143

7.4 Chapter Summary . . . . . . . . . . . . . . . . . . . . . . 144

8 Scattered Interpolation Algorithms and Considerations

8.1 Deformation as Scattered Interpolation. . . . . . . . . . . 147

8.1 .1 Linear Interpolation . . . . . . . . . . . . . . . . 149

$8.1 .2 \quad$ Splines with Tension . . . . . . . . . . . . . . . . . . 151

8.1 .3 Shepard's Method . . . . . . . . . . . . . . . . . 151 
8.1 .4 Radial Basis Functions . . . . . . . . . . . . . . . 152

8.1 .5 Interpolation of 3D Data . . . . . . . . . . . . 159

8.2 Numerical Considerations . . . . . . . . . . . . . . . . . 159

$8.2 .1 \quad$ Regularization . . . . . . . . . . . . . . . . . . 163

8.2.2 Laplacian Spline and Thin-Plate RBF models . . . . . 167

$8.2 .3 \quad$ Detecting Bad Data . . . . . . . . . . . . . . . . . . 169

8.2 .4 Whence the RBF Kernel? . . . . . . . . . . . . . 172

8.2 .5 Green's functions: motivation. . . . . . . . . . . . . 173

8.2 .6 Green's functions . . . . . . . . . . . . . . . . . . 175

$8.2 .7 \quad$ Example derivation of a RBF kernel . . . . . . . . 177

8.3 Chapter Summary. . . . . . . . . . . . . . . . . . . . . 177

III Relationships 179

9 Relationships between various models

$9.1 \quad$ PCA, RBF, and Gaussian Processes . . . . . . . . . . . 182

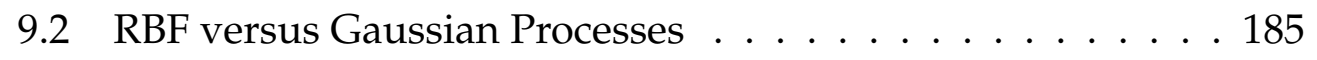

9.2 .1 Outlook . . . . . . . . . . . . . . . . 186

9.3 A Superresolution Extension of PCA . . . . . . . . . . . 187

9.3 .1 Superresolution . . . . . . . . . . . . . . . . 187

9.3 .2 Equivalent kernel . . . . . . . . . . . . . . . . . 189

9.3 .3 Interpretation as evaluation in an RKHS. . . . . . . 189

9.3 .4 Computational Experiment . . . . . . . . . . . . . . 191

9.3 .5 Outlook . . . . . . . . . . . . . . . 193

$9.4 \quad$ PCA and Laplacian Splines $\ldots . .40$.

$9.4 .1 \quad$ PCA . . . . . . . . . . . . . . . . . . . 199

9.4 .2 Laplacian Splines . . . . . . . . . . . . . . . . . . 200

9.4 .3 Relating PCA and Laplacian Splines . . . . . . . . 201

9.4 .4 Outlook . . . . . . . . . . . . . . . . . . . 202

9.5 Chapter Summary . . . . . . . . . . . . . . . . . . . 203 
10 Conclusions 207

10.1 Achieved Objectives . . . . . . . . . . . . . . . . . . . . . . . . . . . . . . . . .

10.2 Limitations . . . . . . . . . . . . . . . . . . . . 208

10.3 Future Work . . . . . . . . . . . . . . . . . . . . 209

Bibliography 211

IV Appendices 241

\begin{tabular}{|l|l|}
\hline A Mathematical Concepts \\
\hline
\end{tabular}

A.1 Interpolation, Approximation, and Regression . . . . . . . . 243

A.2 Parametric versus Non-Parametric . . . . . . . . . . . . . . 244

A.3 Smoothness, Prior, and Model Complexity . . . . . . . . . . 245

A.4 Radial basis functions. . . . . . . . . . . . . . . . . 248

A.5 Laplacian splines . . . . . . . . . . . . . . . . . . . . 249

A.6 Manifold Learning and PCA . . . . . . . . . . . . . 252

A.7 $\quad$ The curse of dimensionality . . . . . . . . . . . . . . . . 256

A.8 $\quad$ Reproducing Kernel Hilbert Space . . . . . . . . . . . . . 261

B Example derivation of a RBF kernel 265 


\section{List of Figures}

2.1 The skeleton subspace deformation algorithm. The deformed

\begin{tabular}{|c|c|}
\hline & position of a point $p$ lies on the line $p^{\prime} p^{\prime \prime}$ defined by the \\
\hline & images of that point rigidly transformed by the neighbor- \\
\hline & ing skeletal coordinate frames, resulting in the characteristic \\
\hline & 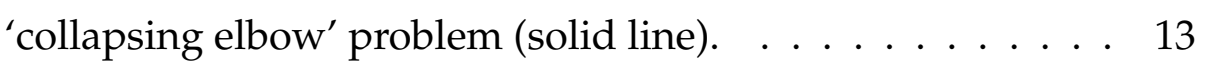 \\
\hline 2.2 & The 'collapsing elbow' in action, c.f. Figure2.1. \\
\hline 2.3 & The forearm in the 'twist' pose, as in turning a door handle, \\
\hline & computed by SSD. As the twist approaches $180^{\circ}$ the arm \\
\hline & collapses. . . . . . . . . . . . . . . . . . . . \\
\hline
\end{tabular}

$3.1 \quad$ Blendshapes are an approximate semantic parameterization of facial expression. From left to right, a half smile, a smile, and a (non-smiling) open-mouth expression. While the smile and open-mouth expressions are most similar in terms of geometric distance, the smile is closer to the half-smile in parameter distance (distance $=0.36$ ) than it is to the open-

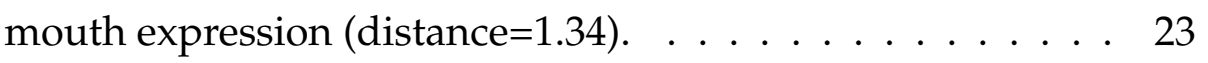

3.2 Blendshapes prevent the artist from making "improper" edits such as this. . . . . . . . . . . . . . . . . 25

3.3 Screenshot of a portion of the blendshape slider interface for a professionally created model. The complete slider interface does not fit on the computer display. This relatively simple model has 45 sliders. . . . . . . . . . . . . . . 26 
3.4 Vector-matrix expression of a blendshape model. $\mathbf{b}_{k}$ de\begin{tabular}{|c|}
\hline notes column $k$ of the matrix, containing the components \\
\hline of each vertex in some order that is consistent across columns. 28 \\
\hline
\end{tabular}

3.5 The basic delta blendshape scheme can be visualized as sit-

\begin{tabular}{|c|}
\hline uating targets at vertices of a hypercube that share an edge \\
\hline with the neutral face at the origin. . . . . . . . . . . 30 \\
\hline
\end{tabular}

3.6 Blendshape targets can be situated at intermediate locations, resulting in piecewise linear interpolation to the full target. 32

3.7 Schematic illustration of the "combination blendshape" idea \begin{tabular}{|c|}
\hline$[174]$. A correction shape (top right) is added with weight \\
\hline$w_{1} \cdot w_{2} \ldots \ldots \ldots \ldots \ldots \ldots \ldots \ldots \ldots \ldots \ldots \ldots \ldots \ldots \ldots \ldots \ldots \ldots \ldots$ \\
\hline
\end{tabular}

3.8 (Left) a third-order combination shape has weight $w_{i} \cdot w_{j}$.

\begin{tabular}{|c|}
\hline$w_{k}$ for some triple $i, j, k$, and so has little effect until the \\
\hline weights approach one. (Right) When intermediate combi- \\
\hline nation shapes are used, the resulting interpolation is not \\
\hline smooth
\end{tabular}

3.9 The "movement matching" principle in [214]. The target

\begin{tabular}{|c|}
\hline cannot fully reproduce source movement (top) due to limi- \\
\hline tations of the target geometry. Attempting to best reproduce \\
\hline the position of each frame results in clipping when the pose \\
\hline is not achievable (dashed line in middle figure). Instead, the \\
\hline movement matching principle attempts to match the tem- \\
\hline poral derivatives, thereby reproducing the shape of the mo- \\
\hline tion (bottom). The red shaded areas indicate the magnitude \\
\hline and distribution of the matching error. . . . . . . . . . 49 \\
\hline
\end{tabular}

3.10 Screenshot of a direct manipulation interface in operation.

\begin{tabular}{|l|}
\hline (Left panel) selecting a point on the model surface creates \\
\hline a manipulator object termed a pin. These can be dragged \\
\hline into desired positions, and the system solves for the slider \\
\hline values (right panel) that cause the face to best match the \\
\hline pinned positions. . . . . . . . . . . . . . . . . . . 54 \\
\hline
\end{tabular}


3.11 Blendshape schemes require that targets are placed at con-

\begin{tabular}{|l|}
\hline strained locations, i.e. the vertices of a "weight hypercube" \\
\hline (Figures 3.5, 3.7). It would be preferable to allow targets to \\
\hline be placed anywhere in face space, allowing the sculpting \\
\hline effort to be directed specifically where it is needed. . . . . . . 60 \\
\hline
\end{tabular}

3.12 Mutual coherence plot for the 46-target blendshape model \begin{tabular}{|c|}
\hline shown in Figure 3.10 and other figures. The $i, j$ entry is the \\
\hline \hline covariance between the $i$-th and $j$-th blendshape targets, i.e. \\
\hline$\frac{\mathbf{b}_{i}^{T} \mathbf{b}_{j}}{\left\|\mathbf{b}_{i}\right\|\left\|\mathbf{b}_{j}\right\|} \cdot \ldots \ldots \ldots \ldots \ldots \ldots \ldots \ldots \ldots \ldots \ldots \ldots \ldots \ldots \ldots \ldots \ldots$ \\
\hline
\end{tabular}

3.13 The space of valid face shapes, represented abstractly as the \begin{tabular}{|c|}
\hline curved shaded region, is approximated as a convex com- \\
\hline bination of a number of blendshapes lying on the bound- \\
\hline ary of the region (black circles). Some regions of the space \\
\hline are not reachable with these blendshapes. This can only be \\
\hline addressed by sculpting blendshapes that lie outside of the \\
\hline valid face space. This is an unnatural task for the modeller. . 66 \\
\hline
\end{tabular}

3.14 Blendshapes appear to function as a sparse basis. This fig\begin{tabular}{|c|}
\hline ure shows a professionally created model with 45 targets, all \\
\hline \hline set to one. Combinations of several (perhaps up to five or \\
\hline \hline so) targets produce useful expressions, but the combination \\
\hline of many targets produces unusable shapes. . . . . . . . . . 67 \\
\hline
\end{tabular}

3.15 Comparison of blendshape (top) and PCA coefficients en\begin{tabular}{|l|}
\hline coding (bottom) of the same 405-frame animation (X-axis) \\
\hline \hline of a 45-dimensional (Y-axis) professionally authored face \\
\hline model. The blendshape coefficients are visibly sparser. . . . 68 \\
\hline
\end{tabular}

3.16 PCA basis vectors are difficult to interpret and remember. These are the 9th and 10th eigenvectors from a professionally produced facial animation. . . . . . . . . . . . 70 
3.17 PCA is a weak "model" of data. From left to right: a syn-

\begin{tabular}{|l|}
\hline thetic data set, the PCA coefficients of this data, the rotated \\
\hline PCA coefficients, and random points having the same co- \\
\hline variance as the data. While the two eigenvectors and cor- \\
\hline responding eigenvalues capture the spread of the data, all \\
\hline \hline the structure ends up in the coefficients. In this two dimen- \\
\hline sional example the coefficients $\mathbf{c}=\mathbf{U}^{T} \mathbf{f}$ are simply a rota- \\
\hline tion of the original data points $\mathbf{f}$, since $\mathbf{U}$ is orthogonal. . . . \\
\hline
\end{tabular}

3.18 Scatterplot of the 1st vs. 3rd PCA coefficients (top) and 2nd

\begin{tabular}{|c|}
\hline vs. 3rd PCA coefficients (bottom) of the professionally-created \\
\hline \begin{tabular}{|l|l|l|} 
405-frame facial animation used in Figure & 3.15 & The plots \\
\end{tabular} \\
\hline show clear non-Gaussian structure. Note that many points \\
\hline are coincident and overlaid in the upper figure. \\
\hline
\end{tabular}

3.19 From A Blendshape Model that Incorporates Physical Interaction [150]. Top row, two meshes to interpolate. Bottom left, linear interpolation. Bottom right, interpolation of edge lengths followed by a mass-spring solve. . . . . . . . . . 77

4.1 (a-c): a blendshape model posed by direct manipulation rather than traditional slider weight editing; (d) a blendshape model posed with a statistical prior, (e) a similar pose from the training animation, (f) an attempt to reproduce this expression without the prior requires more edits and is less successful. . . . . . . . . . . . . 82

4.2 The 9th eigenvector from each of two animated models. . . . 85

4.3 First 10 singular values of the blendshape basis of the model in Figure 4.4 . Although the basis has rank $n$, there are many directions that "almost" define a null space. . . . . . . . . 86 
4.4 Left column: model behavior with unconstrained weights

\begin{tabular}{|l|}
\hline as the target point is dragged to an unreasonable location. \\
\hline Right column: corresponding constrained solutions to this \\
\hline haphazard drag. The green point is the target point location \\
\hline
\end{tabular}
desired by the user. . . . . . . . . . . . . . . . 90

4.5 With a single vertex pinned (top), direct movement of a second vertex is the equivalent of many small slider movements (bottom). However the converse is equally true: a single slider movement is in some cases the equivalent of multiple direct manipulations. Both approaches are desirable. 92

4.6 Scatterplot of selected PCA coefficients of a professional animation with 405 frames. Top: $c_{5}$ versus $c_{6}$, bottom: $c_{10}$ versus $c_{11}$. (also see Figure 3.18). In several animations such as this we found that the lower-numbered coefficients are significantly non-Gaussian. . . . . . . . . . . . . . 95

4.7 Examples of mode snapping. Left column: using Equa\begin{tabular}{|c|}
\hline tion (4.8). Right column: applying mode snapping, Equa- \\
\hline
\end{tabular}

$6.1 \quad$ Face proportions are not strictly Gaussian. Kernel density plots of (left) the distance between the eyes versus the width of the mouth, (right) the width of the mouth versus the \begin{tabular}{|l|l|}
\hline height of the mouth, measured from a database of 359 faces. 115 \\
\hline
\end{tabular}

6.2 The closest distance to the mean among 1000 unit-variance multidimensional Gaussian random variables (vertical axis) as a function of the dimension (horizontal axis). In $100 \mathrm{di}-$ mensions every point in this simulation is more than six standard deviations from the mean. . . . . . . . . . . . . . 116

6.3 Histogram of the angles between all pairs of 100 randomly chosen isotropic Gaussian random variables in 100 dimensions. The angles cluster around $\pi / 2$ : in high dimensions, most data are nearly orthogonal. . . . . . . . . . . . . 117 
6.4 Probability that a sample from a unit variance Gaussian is outside the unit hypersphere for various dimensions. . . . . 117

6.5 Bottom: schematic one dimensional Gaussian distribution, with the area between one and two deviations indicated in

red. This interval is equal to that of the unit radius. Top:

In two dimensions, the area between one and two standard

\begin{tabular}{|c|}
\hline deviations (light blue) is relatively larger than the area of \\
\hline the unit standard deviation disc (light orange). Figure is
\end{tabular}

best viewed in the electronic version of this document. . . . . 119

6.6 The radially integrated Gaussian $N\left(0, \mathbf{I}_{n}\right)$ in various dimen-

\begin{tabular}{|l|}
\hline sions. Each subfigure shows the radially integrated Gaus- \\
\hline \hline sian profile $S_{d-1}(r) G(r)$ (vertical axis) plotted in units of $\sqrt{d}$ \\
\hline (horizontal axis). From left to right: $1,2,10$, and 100 dimen- \\
\hline sions. In high dimensions the probability concentrates in a \\
\hline \hline shell centered at radius $\sqrt{d} . . \ldots \ldots 119$ \\
\hline
\end{tabular}

6.7 Interpolating between a randomly chosen face (left column)

\begin{tabular}{|l|}
\hline and a second face (right column) nearly on the opposite side \\
\hline of the hyperellipse of coefficients. Top row of each image: \\
\hline linear interpolation of coefficients. The middle images lack \\
\hline distinctiveness. Bottom row of each image: interpolating \\
\hline "around the hyperellipse". Detail is preserved throughout \\
\hline the interpolation. . . . . . . . . . . . . . . . . . . . 122 \\
\hline
\end{tabular}

6.8 In this schematic illustration the point along the constraint

\begin{tabular}{|l|}
\hline (dark line) that has the highest probability is the red point. \\
\hline In high dimensions however, the interior of the Gaussian is \\
\hline empty and the probability mass is concentrated toward the \\
\hline outside. . . . . . . . . . . . . . . . . . . . . . 124
\end{tabular}

7.1 Scattered interpolation for skinning. . . . . . . . . . . . 130 
7.2 Comparison of PSD and LBS on an animating shoulder. Top,

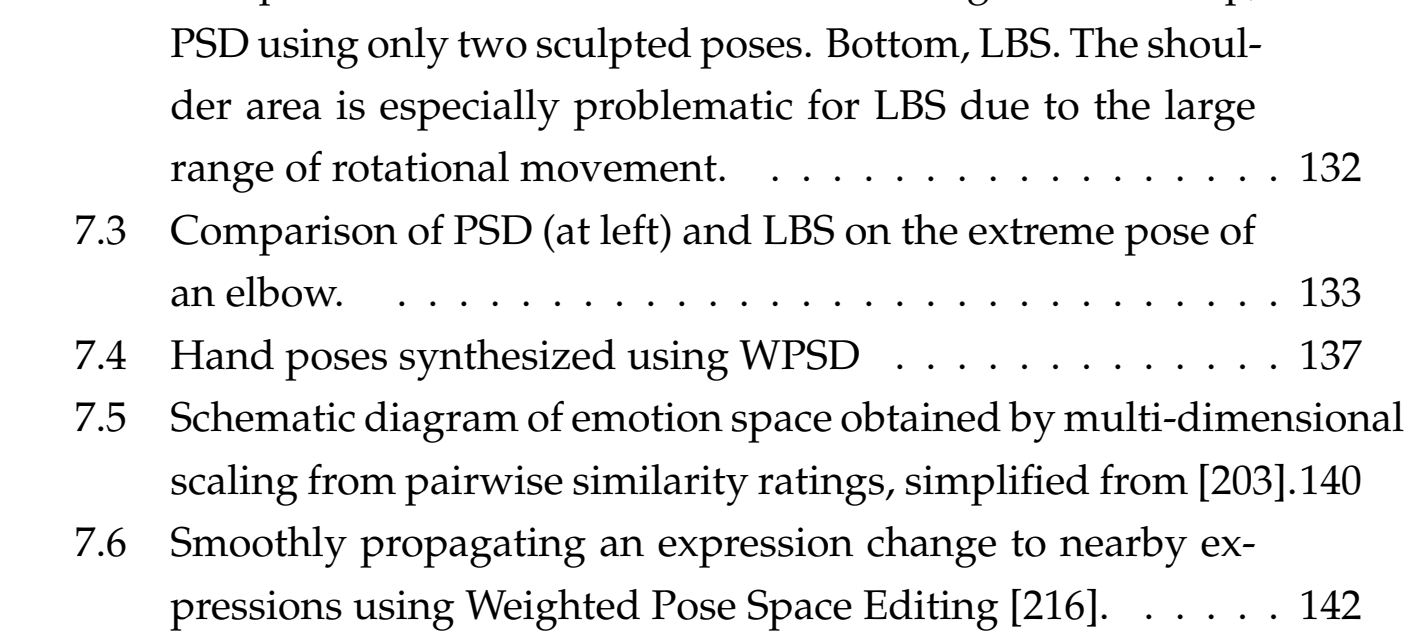

8.1 Shepard's interpolation with $p=2$. . . . . . . . . . . . . . 148

8.2 Shepard's interpolation with $p=1$. . . . . . . . . . . . . . 149

8.3 Spline with tension. Left, cubic spline interpolation. Right, \begin{tabular}{|l|}
\hline cubic spline interpolation of the same points, with tension. \\
\hline The boundary conditions are set to have zero slope (Dirich- \\
\hline
\end{tabular} rlet). . . . . . . . . . . . . . . . . . 151

8.4 Schematic illustration of RBF interpolation in one dimen\begin{tabular}{|l|}
\hline sion. A fixed-width kernel is situated at each data point \\
\hline (solid curves). The weighted sum of these interpolates the \\
\hline given data (dashed line). The weights are obtained by solv- \\
\hline ing a linear system. . . . . . . . . . . . . . . . . . . . . . . 153 \\
\hline
\end{tabular}

8.5 Gaussian-based RBF . . . . . . . . . . . . . . . . 155

8.6 Gaussian-based RBF with different kernel width. . . . . . . . 156

8.7 Gaussian-based RBF with narrow kernel. . . . . . . . . . . . 157

8.8 Normalized RBF interpolation using a Gaussian kernel. The

normalized RBF scheme produces interpolation that is less
sensitive to poor choices of the width parameter. . . . . . . . 158

8.9 Natural cubic spline. . . . . . . . . . . . . . . . 160

8.10 Comparison of the 3D biharmonic clamped plate spline with |Gaussian RBF interpolation . . . . . . . . . . . . . . . 161 
8.11 Regularized cubic spline. . . . . . . . . . . . . . . . . . 164

8.12 Illustration of ill-conditioning and regularization. . . . . . 165

8.13 Ill-conditioning and regularization in 3D. . . . . . . . . 166

8.14 Two examples of a function norm-based approach to automatically identifying bad training data. The point marked with a red star is identified as an outlier. . . . . . . . . . . . 171

9.1 Left: Radial basis interpolation of six points using a Gaussian kernel. Right: equivalent kernels for each of the data points, offset vertically for visibility. . . . . . . . . . . . 189

9.2 Left column: example signals synthesized with covariance $\exp \left(-(15 \Delta)^{2}\right)$. Right column: corresponding superresolu-

\begin{tabular}{|c|}
\hline tion signals. The indicated points are interpolated exactly. \\
\hline$\ldots \ldots \ldots \ldots \ldots \ldots \ldots \ldots \ldots \ldots \ldots \ldots \ldots \ldots \ldots \ldots$ \\
\hline
\end{tabular}

9.3 Left column: signals synthesized with kernel $\cos (18 \Delta) \exp \left(-\Delta^{2}\right)$.

\begin{tabular}{|c|}
\hline Right column: corresponding superresolution signals. The \\
\hline
\end{tabular}
indicated points are interpolated exactly. This kernel illustrates that the superresolution procedure is different from \begin{tabular}{|c|}
\hline spline interpolation. In this case it is adding detail in the \\
\hline
\end{tabular} form of oscillation. . . . . . . . . . . . . . . . . 195

9.4 Inpainting of signals synthesized from the covariance $\exp \left(-(15 \Delta)^{p}\right)$. \begin{tabular}{|c|}
\hline The top plot in each subfigure is the original signal; the bot- \\
\hline \hline tom plot is signal with an omitted section estimated. . . . . 196 \\
\hline
\end{tabular}

9.5 Inpainting of a joint angle signal from motion capture of a walking motion [67]. Each subplot shows one third of the signal omitted and estimated. The covariance is estimated from the original signal (Figure $9.67 .7 \ldots . .197$

9.6 Covariance function estimated from the data used in Fig-

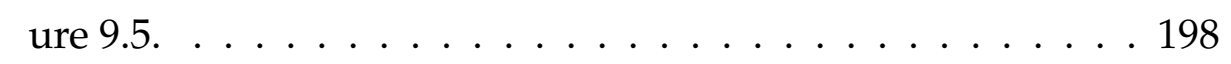


9.7 Left: A reduced-rank PCA model does not exactly fit the

\begin{tabular}{|l|}
\hline data. In this figure the model points are connected with \\
\hline \hline lines for visualization. Right: a spline (in this case a linear \\
\hline spline) fits the data exactly, but does not exclude unreason- \\
\hline able data such as the displaced point in this figure. . . . . . 201 \\
\hline
\end{tabular}

A.1 Laplace interpolation in one dimension is piecewise linear. . 249

A.2 Motivating illustration for PCA: given a collection of 2D

\begin{tabular}{|c|}
\hline data points that are mostly along a line, PCA finds new axes \\
\hline$U$ and $V$. The axis $U$ is the vector that captures the most \\
\hline variance in the data. The data can be summarized or com- \\
\hline pressed by retaining only the single number that describes \\
\hline the position of each point along $U$, rather than the two num- \\
\hline bers that represent the position of each point in $X$ and $Y$. \\
\hline
\end{tabular}

A.3 Histogram of the distances of 1000 100-dimensional pseudorandom variables from the first-generated variable. The pseudorandom variables are uniformly distributed in $0 . .1$ in each dimension. In high dimensions "everything is far away." .................... 257

A.4 High-dimensional effect on pairwise distances. Left, kernel density plot of the pairwise Euclidean distances between $30 N(0,1)$ points in 25 dimensions. Middle, histogram of a scaled exponential RBF kernel applied to these distances, $\exp \left(-\left\|\mathbf{p}_{i}-\mathbf{p}_{j}\right\| / 10000\right)$. Right, a scaled Gaussian RBF kernel applied to these distances, $\exp \left(-\left\|\mathbf{p}_{i}-\mathbf{p}_{j}\right\|^{2} / 10000\right)$. In high dimensions, feature maps and kernels discriminate poorly. . 257 
A.5 Points in high dimensions are situated near a randomly rotated lattice. This figure plots 100 triples of randomly chosen points in a 1000 dimensional space. The plane defined by each triple is transformed to align with the 2D plot with the following procedure: 1) the triple is moved so that the first point is at the origin, 2) the direction from the first point to the second is aligned to the $x=(1,0,0,0,0, \cdots)$ direction using a Householder transformation, 3) lastly, the vector from the first point to the third point is rotated about the $x$ direction to like in the $x, y$ plane. . . . . . . . . . . 258 


\section{Abbreviations and Notation}

PCA Principal Component Analysis

RBF Radial Basis Function

GP Gaussian Process

LS Laplacian Spline

TPS Thin Plate Spline

KNN K-Nearest Neighbors

LBS Linear Blend Skinning

PSD Pose Space Deformation

E Expectation

Vectors are in bold face, and matrices are capitalized bold face letters. An individual element of a vector or matrix uses the corresponding variable without the bold, for example $\mathbf{w}=\left[w_{1}, w_{2}, \cdots, w_{n}\right]$. In a sum, the summation variable and limits are omitted when obvious.

Matlab slicing notation is used in some places. In this notation, $\mathbf{M}_{:, k}$ denotes the $k$ th column of a matrix. 


\section{Chapter 1}

\section{Introduction}

\subsection{Problem Statement and Motivations}

Computer animation is one of the most demanding and labour intensive digital professions. As a general estimate, the creation of a computeranimated movie may require several hundred people working for a period of several years.

The first several decades of computer graphics research largely focused on fundamental questions such as rendering and geometrical representation. There was a large gap between the capabilities of early graphics algorithms and the expressive or photo-real animations desired by artists. This effort has largely succeeded, and now even graphics professionals sometimes have difficulty telling which parts of an image are computergenerated.

Unfortunately this success has not been accompanied by a dramatic reduction in the amount of human labor required. In fact it is difficult to assess the efficiency gains - computers have become vastly faster, but on the other hand expected image complexity has risen in parallel. In any case, human labour is the primary cost in producing a visual effects movie or game. As a result the industry is increasingly migrating to countries with lower wages [112]. 
Improving on this state of affairs is challenging. Many artists probably believe that their craft is one that will never be automated, since it requires artistic judgement. For example, the following might be typical tasks for an artist:

- Make a sky look more "threatening".

- Make a character look exhausted.

- Edit a captured movement to make it appear as though the character is intoxicated.

We agree that these tasks involved perceptual and other "soft" criteria that are outside the realm of complete automation in the near future.

In this thesis, rather than pursuing automation, we will explore a examplebased approach. Character animation will be the particular problem domain considered.

The example-based approach builds on examples of shapes or motion that are manually created by the artist. It exploits the plentiful storage available on modern computers. More importantly, this approach allows substantial artistic input and guidance. It is an "artist in the loop" approach that is fundamentally different from research that produces character movement and deformation entirely using physical simulation. The example-based approach and term have become widespread in computer graphics research in the past 15 years. The author's publication [139] is one of the early milestones of this approach, having received more than 500 citations 1

The challenge in this approach will be to find algorithms that can assemble a set of examples into a manipulable, efficient, and comprehensible "model".

Two insights form the basis for our research:

\footnotetext{
${ }^{1}$ It was also perhaps the first paper to consider character deformation as a highdimensional problem.
} 
1. This "example-based" approach resembles the non-parametric concept in statistics and machine learning. Hence, concepts and techniques from statistical learning may be applied to our problem.

2. Our specific problems of facial and body animation are more usefully posed in an abstract high-dimensional "expression" or "pose" space, rather than the 3-D space (+ time) usually considered in computer graphics.

Following from these insights, we will show that example-based facial and body animation can be usefully considered from the viewpoint of regression, and that associated concepts improve both our understanding of the problem and the quality of the algorithms. We will show that this viewpoint leads to algorithms that allow input and guidance from an artist. The results also improve as more examples are given.

While our approach can be considered as non-parametric regression, it has one key difference: the data is constructed by an artist. This has several implications:

- Since the data is hand-crafted, it will be very limited in quantity perhaps on the order of ten examples, rather than the thousands or millions of data points expected in some machine learning approaches. Thus, we will require methods that generalize from limited examples.

- Our algorithms will have the artist "in the loop" - the artist might adjust a particular example at any time, and expected to rapidly see the resulting model or animation. Thus, approaches that require lengthy training times may not be suitable.

- The nature of "noise" in the data is different. In the case of artistconstructed examples, we usually assume that the data is as intended, or nearly so. In other words, there is no noise in the data. However, even artists make mistakes. When this happens, the error is often 
large or detectable, as happens when two parameters are entered in reverse order, or a model is saved twice under different names (duplicated data).

\subsection{Research Goals}

The preceding comments describe our general philosophy and approach. Applying this thinking to the character animation problem, we have the following specific research objectives:

1. To survey statistical learning concepts that are likely to be applicable to the character animation problem.

2. To analyze facial and body models from a high-dimensional viewpoint.

3. To survey facial animation and body deformation from our examplebased perspective, and demonstrate the applicability of associated statistical learning concepts.

4. To develop new algorithms for character animation that apply statistical learning to our problem.

5. Lastly, to solidify what we have learned by surveying the relationships between several particular techniques.

\subsection{Contributions}

The contributions of the thesis include new algorithms, new analysis and identification of relationships, and definitive surveys, as well as tutorial introductions to some concepts. 
Chapters 3 and 7 provide surveys of facial and body deformation respectively, while indicating relevant learning concepts and methods. Portions of both chapters have appeared in publications.

Chapters 4, 5, and 9 introduce new algorithms, portions of which have appeared in four associated publications. In particular chapter 5 describes the first space-time approach to the important expression cloning problem.

Chapters 6 and 9 complete the thesis research contributions with new analysis and identification of some relationships between several of the underlying mathematical algorithms employed in the thesis.

Throughout the thesis, character deformation is viewed and analyzed as a high-dimensional interpolation or regression problem. Section 3.6 and chapter 6 are focused on the issues arising from this high-dimensional formulation. A survey of statistical learning concepts that are relevant to the character deformation problem is provided in Appendix A.

In addition to the research contributions, the thesis also makes several tutorial contributions. In chapter 8 radial basis functions and splines are related through the Green's function. We give a novel intuitive explanation of this relationship using only the linear algebra that is familiar to graphics people. A more standard derivation involving variational calculus is provided in Appendix B. A derivation of a discrete Poisson problem using a linear algebra-based approach is given in Appendix A. Chapter 3 compares PCA and blendshapes, and uses the relationship to provide an expression of the PCA "prior" for blendshape models.

Specific contributions are listed in chapter order in Section 1.4 below.

\subsection{Organization of the Thesis}

The thesis investigates an example-based approach to several problems in character animation, including problems of both facial and body animation. The thesis is divided into separate sections for the face and the body, together with a third section that identifies some relations between 
the underlying algorithms.

Because each of these topics has its own extensive related literature, the related work is surveyed together with the topic in each chapter. Chapter 2 provides a brief and general overview of character deformation algorithms. An appendix provides a survey of required mathematical and machine learning concepts that may be less familiar to readers in the computer graphics field.

Chapters begin with a statement of context and how the chapter fits in with the thesis as a whole, followed by an introduction to the contents of the chapter. A chapter summary recaps the main contents and contribution of each chapter.

The contents and specific contributions of each chapter are listed next:

- Chapter 2 provides a brief and general overview of character skin deformation algorithms, focusing on existing approaches that are alternative to the example-based approach championed in this thesis.

- Chapter 3 gives a comprehensive survey and analysis of blendshape facial models, including an exploration of blendshapes as a parameterization, and a thorough comparison to principal component analysis (PCA).

Part of the work in this chapter was published in the following papers:

J.P. Lewis, Ken Anjyo, Taehyun Rhee, Mengjie Zhang, Frederic Pighin, Zhigang Deng, Survey and Theory of Blendshape Facial Animation Computer Graphics Forum, submitted for publication, 2015.

J.P. Lewis, Ken Anjyo, Taehyun Rhee, Mengjie Zhang, Frederic Pighin, Zhigang Deng, Practice and Theory of Blendshape Facial Models, Eurographics 2014.

- Chapter 4 introduces an inverse kinematic-like editing algorithm for blendshape facial models. The algorithm allows editing to be guided 
by training data from previous animations.

The major result in section 4.3 is original unpublished work. Other parts of the work in this chapter were published in the following papers:

Ken Anjyo, Hideki Todo, J.P. Lewis A Practical Approach to Direct Manipulation Blendshapes, Journal of Graphics Tools, Volume 16, Issue 3, 2012.

Ozan Cetinaslan, Vernica Orvalho, John Lewis, Sketch-Based Controllers for Blendshape Facial Animation, Eurographics 2015.

- Chapter 5 introduces the first space-time algorithm for the expression cloning problem. This algorithm brings the power of Poisson editing to motion capture retargeting.

This chapter is original research. Part of the work in this chapter was published in the following paper:

Yeongho Seol, J.P. Lewis, Jaewoo Seo, Byungkuk Choi, Ken Anjyo, Junyong Noh, Spacetime Expression Cloning for Blendshapes ACM Transactions on Graphics, April 2012.

- Chapter 6 analyzes high-dimensional aspects of the facial modeling and animation problem, and arrives at the conclusion that the popular maximum a posteriori (MAP) estimate is not justified in the case of Gaussian distributions in high dimensions.

This chapter is original research. Part of the work in this chapter was published in the following paper:

J.P. Lewis, Zhenyao Mo, Ken Anjyo, Taehyun Rhee, Probable and improbable faces, Mathematical Progress in Expressive Image Synthesis, Springer, 2014.

- Chapter 7 puts the pose-space deformation algorithm in its proper place as a form of non-parametric regression, and makes connections 
to core concepts in statistical learning. This chapter provides survey and analysis.

- Chapter 8 describes the implementation of example-based skinning using scattered interpolation algorithms, including some algorithmic and numerical issues. This chapter is tutorial in nature, and the selection of topics is based on practical experience with the algorithm presented in chapter 7. A more comprehensive survey of scattered interpolation for computer graphics is provided in the author's recent ACM courses [137, 107, 9].

- Chapter 9 explores several further relationships:

- We show a close relationship between radial basis function regression and Gaussian processes.

- We explore a relationship between the eigen-expansion of the radial basis kernel and PCA, leading to a hybrid scheme that provides a superresolution for PCA.

- We develop a partial relationship between PCA and splines, motivated by the goal of developing a unified model that has the best properties of both.

This chapter is primarily analysis and interpretation. Portions of the work in this chapter were published in the following papers:

J.P. Lewis, Ken Anjyo, Taehyun Rhee, Superresolution from Principal Component Models by RKHS Sampling, in Mathematical Progress in Expressive Image Synthesis II, Springer, 2015.

J.P. Lewis, Taehyun Rhee, Mengjie Zhang, Principal Component Analysis and Laplacian Splines: Steps toward a Unified Model, in Wakayama et al., The Impact of Applications on Mathematics, Springer, 2014. 


\section{Chapter 2}

\section{Background}

This chapter provides a brief review of the subject of deformation for computer graphics character animation. The focus here is on existing approaches that are alternative to the example-based approach championed in this thesis. Research that specifically underlies the contributions of the thesis is surveyed in more detail in the appropriate location, primarily in Chapters 3 (faces) and 7 (body).

For a tutorial survey of concepts from machine learning that may be less familiar in graphics, including indication of places where they appear in the thesis, please refer to Appendix A. Particular definitions are expanded as needed in later chapters.

\subsection{Deformation for Character Animation}

The movement of a computer graphics character is controlled by the animator using a set of high level control parameters. A character "rig" is a relatively small set of parameters that provide as much control as possible [173, 157]. Examples include:

- the joint angles of an abstract skeleton

- weights on a basis representing facial expressions 
- the vertices of a coarse cage surrounding the model.

Given these parameters, the animation software must calculate the shape of the moving skin of the character. This problem is sometimes referred to as mesh deformation.

For our purpose, deformation algorithms may be categorized into generic techniques, custom parametric methods, physically-based methods, bonebased skinning methods, facial animation methods, and the example-based methods explored in this thesis. This categorization is based in part on historical usage and viewpoints and terminology in the animation community.

\subsubsection{Generic algorithms}

Free-form deformation methods can be employed to deform computer graphics characters. We term these "generic" algorithms, since they can be applied to any sort of model and are not specifically targeted at characters. Examples of these methods include free-form deformation [211, 103], Wires [221], cage-based deformers [109, 146, 128] and other multiresolution representations [89], swirling sweepers for constant-volume deformation [6], and others. These algorithms permit arbitrary non-physical deformations and so are most useful for cartoon characters. While in concept they could be used for realistic character animation, the do not guarantee physical-looking deformation (e.g. quasi-rigidity of the limbs) and thus the artist must make extra effort to obtain realistic results.

A productive recent research area in computer graphics focuses on deformation algorithms that approximately preserve selected properties (such as rigidity) of the pointwise transformations relating the original and deformed models [2, 223]. These approaches are typically formulated for two or three dimensions and assume that an underlying mesh structure is being deformed. This likely prevents their use in high dimensions, at least without significant further research. For example, the as-rigid-as- 
possible (ARAP) family algorithms explicitly represent transformations of neighboring vertices on the mesh.

\subsubsection{Parametric approaches}

In contrast to generic deformation approaches, parametric deformation uses algorithms that target specific aspects of the character animation problem 1 Continuous deformation of a character skin was first addressed in Parke's pioneering facial animation work [181]. In this work, control vertices were deformed by custom algorithmic implementation of carefully selected high-level parameters ('raise-upper-lip', etc.). An example of the parametric deformation category in body animation is [116], which defined a simple custom algorithm to produce elbow deformation including the skin crease on the acute side of the joint (the parameter here is the joint angle).

On the basis of its definition, this "parametric" approach might be considered to subsume all other approaches. However, we will consider it to refer to custom algorithms other than one of the common algorithms (linear blend skinning, blendshapes, etc.). The author believes that such custom deformers are frequently used in modern character animation, particularly for Disney/Pixar style cartoon animation.

\subsubsection{Physically-based approaches}

Physically-based deformation approaches produce the shape of a character's skin using physical simulation, typically involving the underlying muscles. Research in this area spans several decades [187, 154, 56, 235, 168, 249, 207, 111]. Terzopoulos and Waters [231] pioneered physically-based

\footnotetext{
${ }^{1}$ Here we are following Parke's usage of this (overloaded) term. "Rigging" is a modern term that encompasses a collection of special-purpose algorithmic deformations applied together with more general algorithms such as skinning [173].
} 
facial animation. This work also included a simple tracking component. The recent state of the art in this area is probably [219].

In addition to physically based simulation approaches, there are also approaches that draw on ideas and algorithms from physics without attempting to simulate the full "stack" of bone, muscle, and skin at some resolution. Examples of such "physically inspired" approaches include [140], which computes skin stretching without simulating the underlying muscles.

Physical simulation obviously requires knowledge of physics in addition to advanced numerical methods, and the author lacks the knowledge to organize and comment on this literature. We leave this to surveys and courses such as [167, 14]. Nevertheless, it is possible to contrast the general approach with the the example-based approach explored in this thesis:

- Physical simulation has the promise of ultimate realism.

- Physical simulation can greatly reduce the human effort required to produce a character model. For example, an example-based approach requires dozens of shapes to describe mouth movement, and cannot reasonably accommodate collision and compression of the lips - hundreds of shapes would be required to approximate all the possible shapes resulting from pressing the lips together in various ways

- On the other hand, physical simulation of high-resolution character models may not be possible at interactive frame rates, whereas movie-resolution example-based models can be played in real-time.

- Example-based models allow the artist to directly specify the desired character deformation, whereas physical simulation is an indirect process (specify muscle shapes and activations; run the simulation). Achieving a particular desired deformation with physical simulation might require many trial-and-error iterations. 


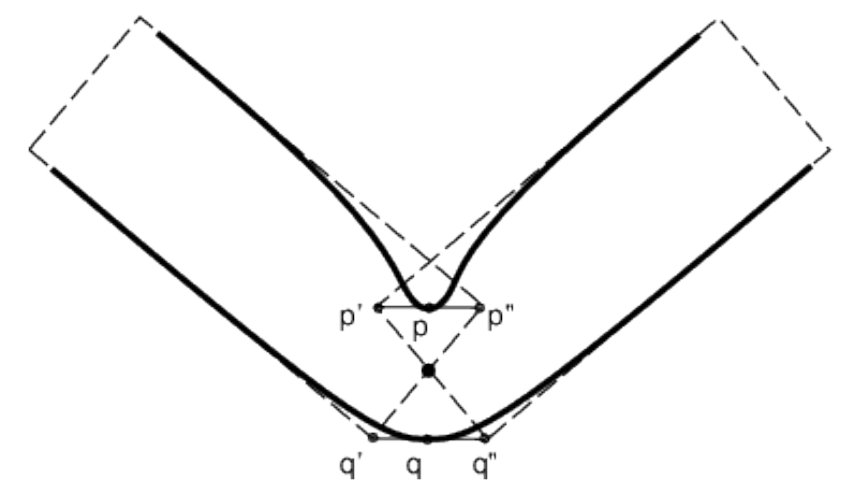

Figure 2.1: The skeleton subspace deformation algorithm. The deformed position of a point $p$ lies on the line $p^{\prime} p^{\prime \prime}$ defined by the images of that point rigidly transformed by the neighboring skeletal coordinate frames, resulting in the characteristic 'collapsing elbow' problem (solid line).

Many computer graphics characters are fantasy characters (aliens, dragons, etc.), and one could argue that these are outside the scope of physical simulation. On the other hand, there is an anthropomorphic principle that most fantasy creatures are only slightly removed from human experience - aliens are almost always humanoid, dragons are big lizards, etc. - and thus physical simulation is applicable. Indeed, physical simulation has been used on some of the characters in recent fantasy movies [66].

\subsubsection{Skinning}

Skinning typically refers to a process in which the character mesh is deformed as a function of the motion of an underlying abstract skeleton. The first and most widely used skinning algorithm is called linear blend skinning (LBS) in the research literature (it is also known as skeleton subspace deformation (SSD) in some older papers). It is implemented in commercial animation software under a variety of uninformative names such as skinning, enveloping, boning, etc. The algorithm is unpublished but the idea 


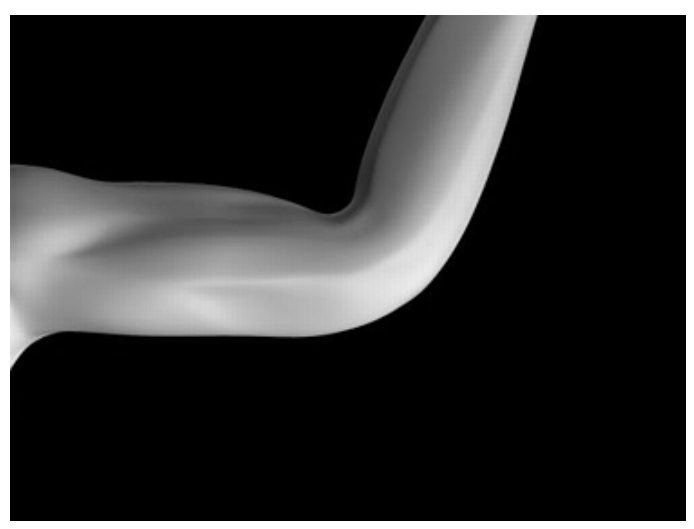

Figure 2.2: The 'collapsing elbow' in action, c.f. Figure2.1.

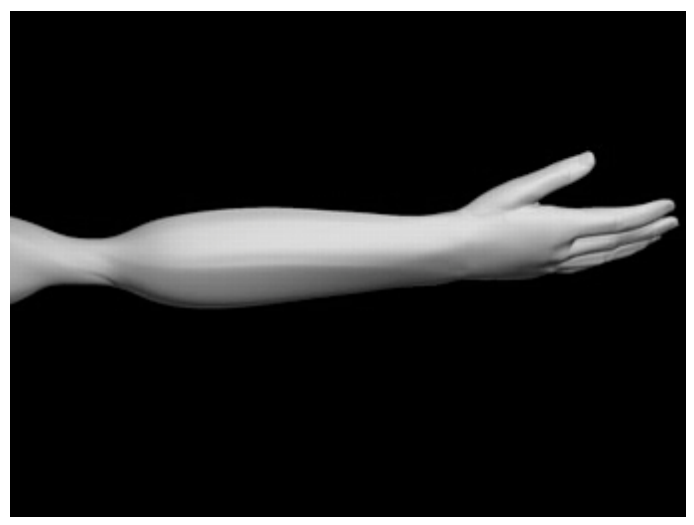

Figure 2.3: The forearm in the 'twist' pose, as in turning a door handle, computed by SSD. As the twist approaches $180^{\circ}$ the arm collapses. 
is simple and it appears as a component of early research such as [153].

In LBS the position of a control vertex $p$ on the deforming surface of an articulated object is a weighted sum of the transformed positions of that vertex, where the transforms are those of some number of surrounding "bones" (Figure 2.1p.

This may be notated

$$
\overline{\mathbf{p}}=\sum w_{k} \mathbf{L}_{k} \mathbf{p}
$$

(in more detail)

$$
\overline{\mathbf{p}}=\sum w_{k} L_{k}^{\delta} L_{k}^{0-1} L_{\mathbf{p}}^{0} \mathbf{p}
$$

where $L_{p}^{0}$ is the transform from the surface containing $\mathbf{p}$ to the world coordinate system, $L_{k}^{0}$ is the transform from the stationary skeletal frame $k$ to the world system $\left(L_{k}^{0-1} L_{p}^{0}\right.$ together represent $\mathbf{p}$ in the coordinate system of skeletal frame $k$ ), and $L_{k}^{\delta}$ expresses the moving skeletal frame $k$ in the world system. The deformation is controlled by the user through the "skinning weights" $w_{k}$.

LBS is simple and versatile. For example, secondary animation effects such as muscle bulging and swelling of the chest can be achieved in the LBS framework by weighting the surface to an abstract "bone" whose translation or scale is manually animated.

The major shortcoming of LBS results directly from the fact that the deformation is restricted to the "subspace" spanned by the transformed vertices (Figures 2.1). In common situations such as shoulders and elbows the desired deformation does not lie in this subspace, hence no amount of adjusting the algorithm weights will produce good results. In fact, the LBS algorithm can be easily identified in animations by its characteristic 'collapsing joint' defect (Figures 2.12 2.2.

This problem is extreme in the case of simulating the twist of a human forearm (the pose taken in turning a door handle, Figure 2.3). In this case the subspace basis consists of surface points rigidly transformed by the forearm frame (no axis rotation) and the wrist frame (axis rotation). With a rotation of 180 degrees this line crosses the axis of the arm, i.e., the forearm 
collapses entirely as the LBS weights transition at some point from the forearm to wrist frames. In practice, these artifacts have been corrected by layering other deformers and rigging expressions, or even by manual re-sculpting.

A second shortcoming of LBS is that the parameterization presented to the artist is somewhat indirect: rather than simply specifying the desired shape, the artist must paint a number of weight maps, one for each bone influencing a region. Reasonable default weights can be produced by automatic algorithms [15], however if the resulting deformation is not exactly as desired, the artist is still faced with adjusting weights. LBS consequently has the reputation for being tedious and difficult to control. Artists with a poor understanding of the underlying algorithm have difficulty distinguishing between results that can be further improved by adjusting weights and results that cannot be improved since the desired result lies outside the achievable subspace. This fact leads to considerable frustration, since it is possible to believe that that some as yet undiscovered combination of weights will produce better results.

Consideration of the limitations of LBS led to dual quaternion skinning (DQS) [113]. DQS largely solves the collapsing elbow and twist artifacts of LBS, and has superseded several other improved algorithms [244, 163, 158]. DQS has also contributed to study and awareness of the nature of rotation (and transformations more generally) within the graphics community [10, 175].

Another approach to skinning is implicit skinning [35, 236, 237]. In these algorithms the skin surface is defined as an implicit surface offset from the underlying skeleton. As is the case with LBS and DQS, these may produce somewhat generic "tube-like" deformations without anatomical detail, but have the advantage that they can mimic collision effects and the resulting bulging to mimic volume conservation [236, 237]. The recent state of the art produces quite realistic deformations [236] and does not need the weight editing required in other skinning methods. How- 
ever, detailed editing and adjustment is not provided within the implicit skinning framework, so the results must be accepted "as is".

To distinguish the skinning algorithms mentioned above from the example-based skinning approach described later, we term the LBS and DQS skinning algorithms as "formulaic" skinning approaches, in that they produce the deformation using closed-form mathematical formulas. Any of these skinning algorithms (LBS, DQS, implicit) can be used as an underlying first approximation to reduce the number of examples needed in example-based skinning (Chapter 7), and doing so addresses the editability issue identified above.

\subsection{Chapter Summary}

This chapter briefly surveyed character skin deformation algorithms, focusing on existing approaches that are alternative to the example-based approach explored in this thesis. 


\section{Part I}

\section{Linear Models}

Linear "blendshape" models are widely used in facial animation. This first part of the thesis defines and surveys the blendshape model. We analyze the advantages of this approach relative to orthogonal principal component models, as well as limitations of the blendshape approach. Two new algorithms designed for accelerated editing of blendshape facial animations are introduced. 


\section{Chapter 3}

\section{Survey and Analysis of Linear Facial Models}

'Blendshapes", a simple linear model of facial expression, is the prevalent approach to realistic facial animation. It has driven animated characters in Hollywood films, and is a standard feature of commercial animation packages. The blendshape approach originated in industry, and became a subject of academic research relatively recently. This chapter describes the published state of the art in this area, covering both literature from the graphics research community, and developments published in industry forums. We show that, despite the simplicity of the blendshape approach, there remain open problems associated with this fundamental technique.

This chapter is divided into survey and analysis. Sections 3.1-3.5 provide a comprehensive survey of blendshape facial modeling. In particular we will:

- Survey the linear "blendshape" model

- Introduce the mathematical notation that will be used in the next chapters

- Briefly introduce the topics of the following chapters, placing them in context 
Sections 3.6 and 3.7 analyze the blendshape approach from the point of view of interpolation and parameterization, suggesting new insights and open questions.

\section{Introduction}

The face has always held a particular interest for the computer graphics community: its complexity is a constant challenge to our increasing ability to model, render, and animate lifelike synthetic objects. A variety of approaches to facial animation have been pursued, including:

- parametric models [179, 180], in which custom deformation algorithms defined specifically for the face are implemented,

- approaches using proprietary deformers of commercial packages, such as "cluster deformers" [232],

- physically-based models, which approximate the mechanical properties of the face such as skin layers, muscles, fatty tissues, bones, etc. [231, 219],

- meshes driven by dense motion capture [84, 99, 38, 165, 43, 19],

- principal component analysis (PCA) models obtained from scans or motion capture [33, 34],

- approaches based on spatial interpolation [28] or interpolation in an abstract "pose" or expression space [139, 29, 127, 200],

- "blendshape" models, which are referred to with several other names (refer to the Terminology section), and

- hybrid approaches [117]. 

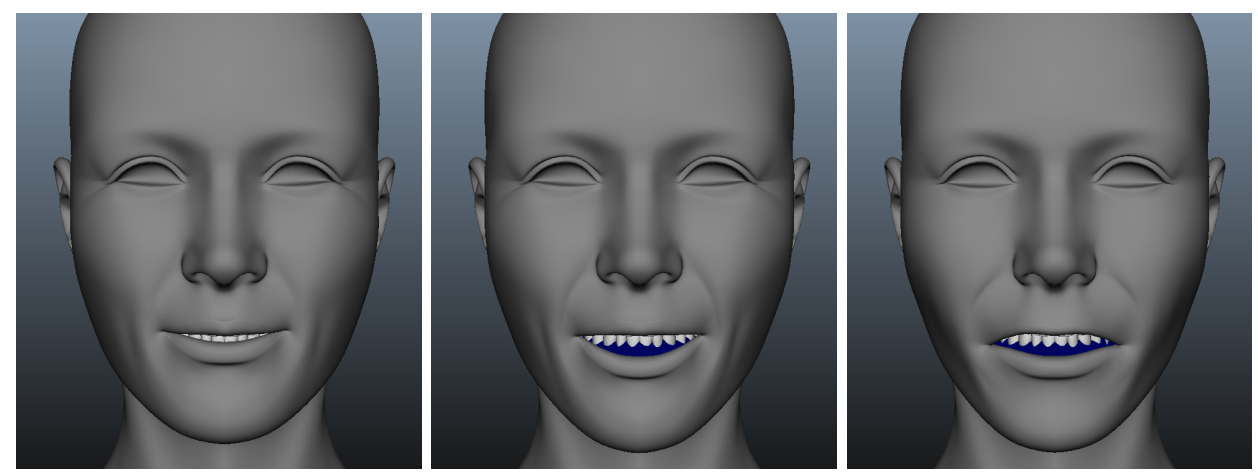

Figure 3.1: Blendshapes are an approximate semantic parameterization of facial expression. From left to right, a half smile, a smile, and a (nonsmiling) open-mouth expression. While the smile and open-mouth expressions are most similar in terms of geometric distance, the smile is closer to the half-smile in parameter distance (distance $=0.36$ ) than it is to the open-mouth expression (distance $=1.34$ ).

See [173, 74, 182, 170] for further overview of facial animation approaches.

Among these choices, blendshapes remain popular due to the combination of simplicity, expressiveness, and interpretability. Blendshape facial animation is the predominant choice for realistic humanoid characters in the movie industry. The approach has been used for lead characters in movies such as The Curious Case of Benjamin Button [87], King Kong [204], The Lord of the Rings [220], Final Fantasy: The Spirits Within, and Stuart Little. Even when more sophisticated approaches to facial modeling are used, blendshapes are sometimes employed as a base layer over which nonlinear or physically based deformations are layered.

A blendshape model generates a facial pose as a linear combination of a number of facial expressions, the blendshape "targets". Each target can be a complete facial expression, or a "delta" expression such as raising one of the eyebrows. The Facial Action Coding System [81] has been used to guide the construction of the target shapes [204, 101]. Many of the targets 
in this system approximate the linearized effect of individual facial muscles. By varying the weights of the linear combination, a range of facial expressions can be expressed with little computation. The set of shapes can be extended as desired to refine the range of expressions that the character can produce. In comparison with other representations, blendshapes have several advantages that together explain the popularity of this technique:

- The desired shape of the face can be directly specified, by sculpting the blendshape targets. Other approaches provide indirect control over shape.

- Blendshapes are a semantic parameterization: the weights have intuitive meaning for the animator as the strength or influence of the various facial expressions (Figure 3.1). Other linear models such as PCA do not provide this (section 3.7.7.

- To some extent blendshapes force the animator to stay "on model", that is, arbitrary deformations are not possible (Figure 3.2). While this could be seen as limiting the artist's power, it helps ensure that the facial character is consistent even if animated by different individuals. It also enforces a division of responsibility between the character modeler and animator.

Although the blendshape technique is conceptually simple, developing a blendshape face model is a large and labor intensive effort at present. To express a complete range of realistic expressions, digital modellers often have to create large libraries of blendshape targets. For example the character of Gollum in the Lord of the Rings had 675 targets [88, 220]. Generating a reasonably detailed model can be as much as a year of work for a skilled modeler, involving many iterations of refinement.

The remainder of this chapter is organized as follows. The first three sections define our subject, while subsequent sections describe particular 


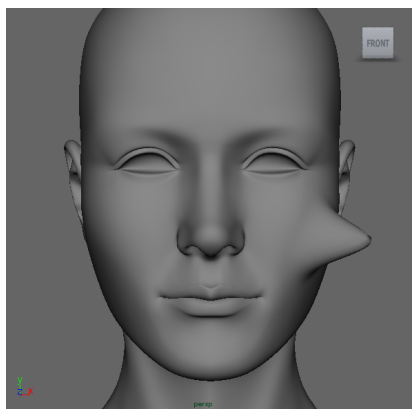

Figure 3.2: Blendshapes prevent the artist from making "improper" edits such as this.

topics and summarize associated research and open problems. Section 3.1 collects the industry terminology of blendshapes. Section 3.2 presents a brief history, though most related literature will be discussed in relevant later sections. Section 3.3 describes blendshapes from a linear algebra point of view, including recent variants such as "combination" blendshapes. Section 3.4 surveys methods of constructing blendshape models, including model transfer and refinement of models. Section 3.5 reviews interaction and animation techniques including performance-driven and direct manipulation approaches. Section 3.6 considers blendshapes as a high-dimensional interpolation problem. Section 3.7/considers blendshapes as a parameterization, and contrasts this approach with those based on principal component analysis. Section 3.8 mentions several extensions of the blendshape idea.

\subsection{Terminology}

The "blendshapes" term was introduced in the computer graphics industry, and we follow that definition: blendshapes are linear facial models in which the individual basis vectors represent individual facial expressions. As a consequence the basis is not orthogonal in general. The individual basis vectors have been referred to as blendshape targets and morph targets, 


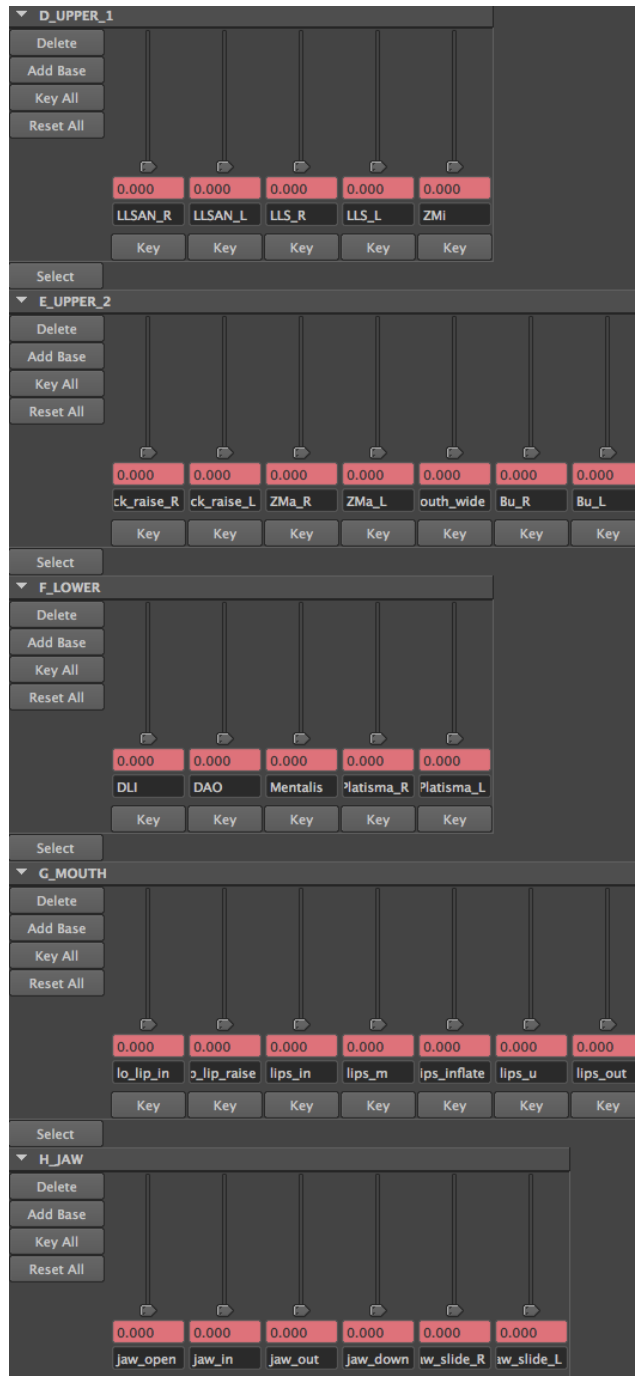

Figure 3.3: Screenshot of a portion of the blendshape slider interface for a professionally created model. The complete slider interface does not fit on the computer display. This relatively simple model has 45 sliders. 
or (confusingly) as shapes or blendshapes. The corresponding weights are often called sliders, since this is how they appear in the user interface (Figure 3.3. . A morphable model [33] is also a linear facial model, though it may focus on identity rather than expression, and its underlying basis is orthogonal rather than semantic.

From an artist's point of view, the interpretability of the blendshape basis is a defining feature. To manage the scope of this survey we will not attempt to fully survey techniques that make use of an orthogonal basis. Since the distinction is less important from a mathematical and programming point of view, however, relevant concepts that have to date only been employed with orthogonal models will be mentioned.

\subsection{History}

The origin of the blendshape approach is not generally associated with an academic publication, though it was well known in the computer graphics industry by the 1980s. Although Fred Parke is known for his pioneering work on the alternate parametric approach to facial modeling [178, 179], he experimented with linear blending between whole face shapes [177]. By the late 1980s the "delta" or offset blendshape scheme became popular [20] and appeared in commercial software [24, 83]. In this variant a neutral face shape is designated and the remaining shapes are replaced by the differences between those shapes and the neutral shape. This results in localized control when the differences between the target shape and the neutral face are restricted to a small region, although it relies on the modeler to produce shapes with this property.

This idea was extended to a segmented face where separate regions are blended independently [115], thus guaranteeing local control. A standard example is the segmentation of a face into an upper region and a lower region: the upper region is used for expressing emotions, while the lower region expresses speech [72]. 


$$
\left[\begin{array}{c}
f_{1 x} \\
f_{1 y} \\
f_{1 z} \\
f_{2 x} \\
f_{2 y} \\
\vdots \\
\vdots \\
\vdots \\
\vdots \\
\vdots \\
f_{p z}
\end{array}\right]=\left[\begin{array}{cccc}
x & x & \cdots & x \\
x & x & \cdots & x \\
y & y & \cdots & y \\
\vdots & \mid & \cdots & \mid \\
\mathbf{b}_{1} & \mathbf{b}_{2} & \cdots & \mathbf{b}_{n} \\
\mid & \mid & \cdots & \mid \\
\mid & \mid & \cdots & \mid \\
\mid & \mid & \cdots & \mid \\
\mid & \mid & \cdots & \mid
\end{array}\right]\left[\begin{array}{c}
w_{1} \\
w_{2} \\
\vdots \\
w_{n}
\end{array}\right]
$$

Figure 3.4: Vector-matrix expression of a blendshape model. $\mathbf{b}_{k}$ denotes column $k$ of the matrix, containing the components of each vertex in some order that is consistent across columns.

While blendshape targets are most often considered as time-independent facial expressions, it is also possible to view individual blendshapes as being situated at particular times in the animation, and to simply cross-fade between them to produce the final animation. This time-dependent blendshape approach provides the most direct control possible by guaranteeing that desired expressions appear at particular points in the animation, but it requires the construction of many blendshapes that may not be reusable at other points in the animation. Some animations have combined the time-dependent and time-independent blendshape approaches [262].

Additional literature on blendshapes will be mentioned in appropriate sections of the remainder of the chapter. 


\subsection{Algebra and Algorithms}

Some insight and ease of discussion can be had by viewing blendshapes as a simple vector sum. To be concrete, consider a facial model composed of $n=100$ blendshapes, each having $p=10000$ control vertices ("points"), with each vertex having three components $x, y, z$. By "unrolling" the numbers composing each blendshape into a long vector $\mathbf{b}_{k}$ in some order that is arbitrary (such as xxxyyyzzz, or alternately xyzxyzxyz) but consistent across the individual blendshapes (Figure 3.4), the blendshape model is expressed as

$$
\mathbf{f}=\sum_{k=0}^{n} w_{k} \mathbf{b}_{k}
$$

or using matrix notation

$$
\mathbf{f}=\mathbf{B w}
$$

where $\mathbf{f}$ is the resulting face, in the form of a $m=30000 \times 1$ vector $(m=3 p)$, $\mathbf{B}$ is a $30000 \times 100$ matrix whose column vectors, $\mathbf{b}_{k}$, are the individual blendshapes $(30000 \times 1$ vectors), and $\mathbf{w}$ are the weights $(\mathrm{a} 100 \times 1$ vector). We take $b_{0}$ to be the blendshape target representing the neutral face. This linear algebra viewpoint will be used to describe various issues and algorithms.

Equation (3.2) represents the global or "whole-face" blendshape approach. In this approach scaling all the weights by a multiplier causes the whole head to scale. Overall scaling of the head is more conveniently handled with a separate transformation, however. To eliminate undesired scaling the weights in equation (3.2) may be constrained to sum to one. Additionally the weights can be further constrained to the interval $[0,1]$, as described in section 3.7.5.

\subsubsection{Delta blendshape formulation}

In the "delta" blend shape formulation, one face model $b_{0}$ (typically the resting face expression) is designated as the "neutral" face shape, and the 


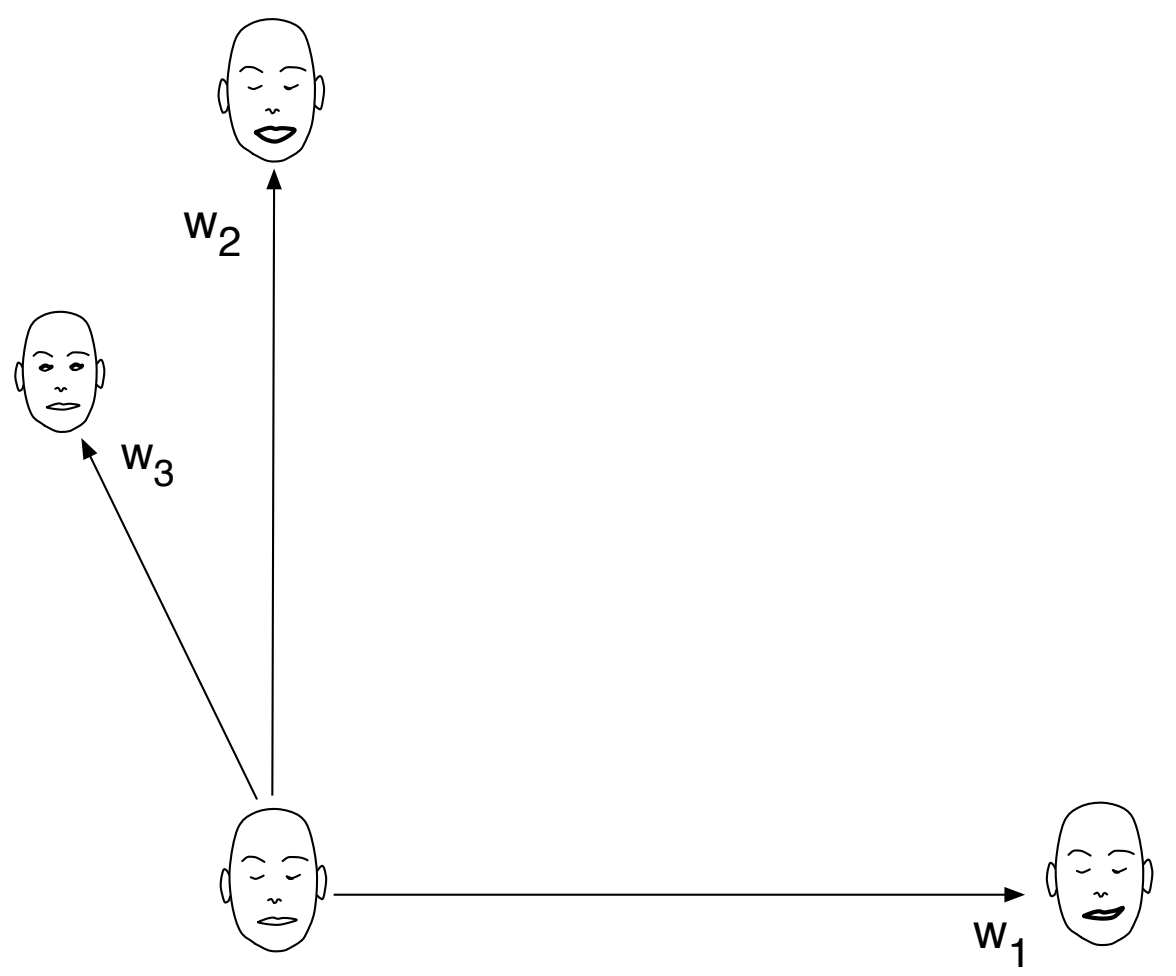

Figure 3.5: The basic delta blendshape scheme can be visualized as situating targets at vertices of a hypercube that share an edge with the neutral face at the origin. 
remaining targets $\mathbf{b}_{k}, \quad k=1 \ldots n$ are replaced with the difference $\mathbf{b}_{k}-\mathbf{b}_{0}$ between the $k$ th face target and the neutral face:

$$
\mathbf{f}=\mathbf{b}_{0}+\sum_{k=1}^{n} w_{k}\left(\mathbf{b}_{k}-\mathbf{b}_{0}\right)
$$

(with $\mathbf{b}_{0}$ being the neutral shape). We denote this as

$$
\mathbf{f}=\mathbf{b}_{0}+\mathbf{B w}
$$

(note that we are reusing variable names from equation (3.2)). In this formulation the weights are conventionally limited to the range $[0,1]$, although there are exceptions to this convention. For example the Maya [232] blendshape interface allows the [0,1] limits to be overridden by the artist if needed.

If the difference between a particular blend shape $\mathbf{b}_{k}$ and the neutral shape is confined to a small region, such as the left eyebrow, then the resulting parameterization offers intuitive localized control.

The delta blendshape formulation is used in popular packages such as Maya, and our discussion will assume this variant if not otherwise stated. Many comments apply equally (or with straightforward conversion) to the whole-face variant.

A blendshape model can be considered as placing targets at some of the vertices of a $n$-dimensional hypercube, with the origin being the neutral shape, and hypercube edges representing weights on the corresponding targets (Figure 3.5). Note that this and following figure are schematic, with a small face image representing the collection of vertex components of a particular blendshape target.

\subsubsection{Intermediate shapes}

As an individual weight in Eq. (3.4) varies from zero to one, the moving vertices on the face travel along a line. To allow more fidelity, production blendshape implementations such as that in Maya [232] allow targets to 


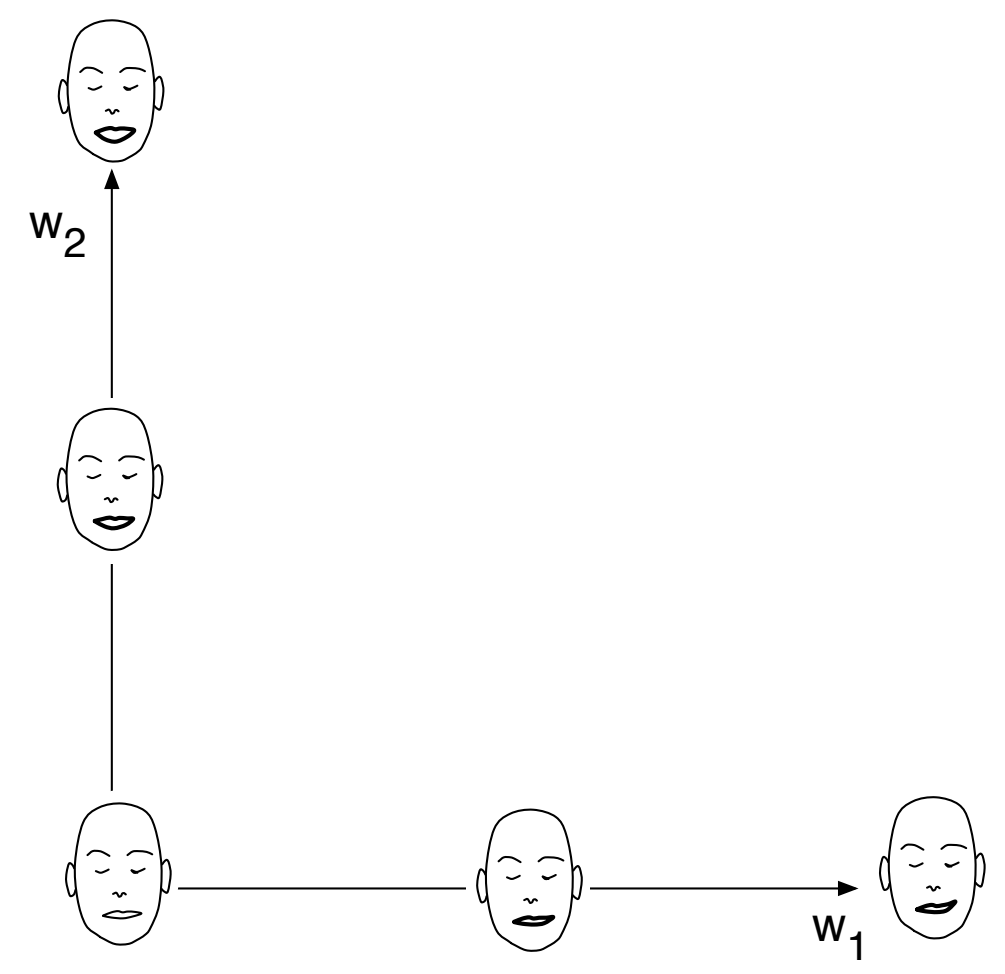

Figure 3.6: Blendshape targets can be situated at intermediate locations, resulting in piecewise linear interpolation to the full target. 


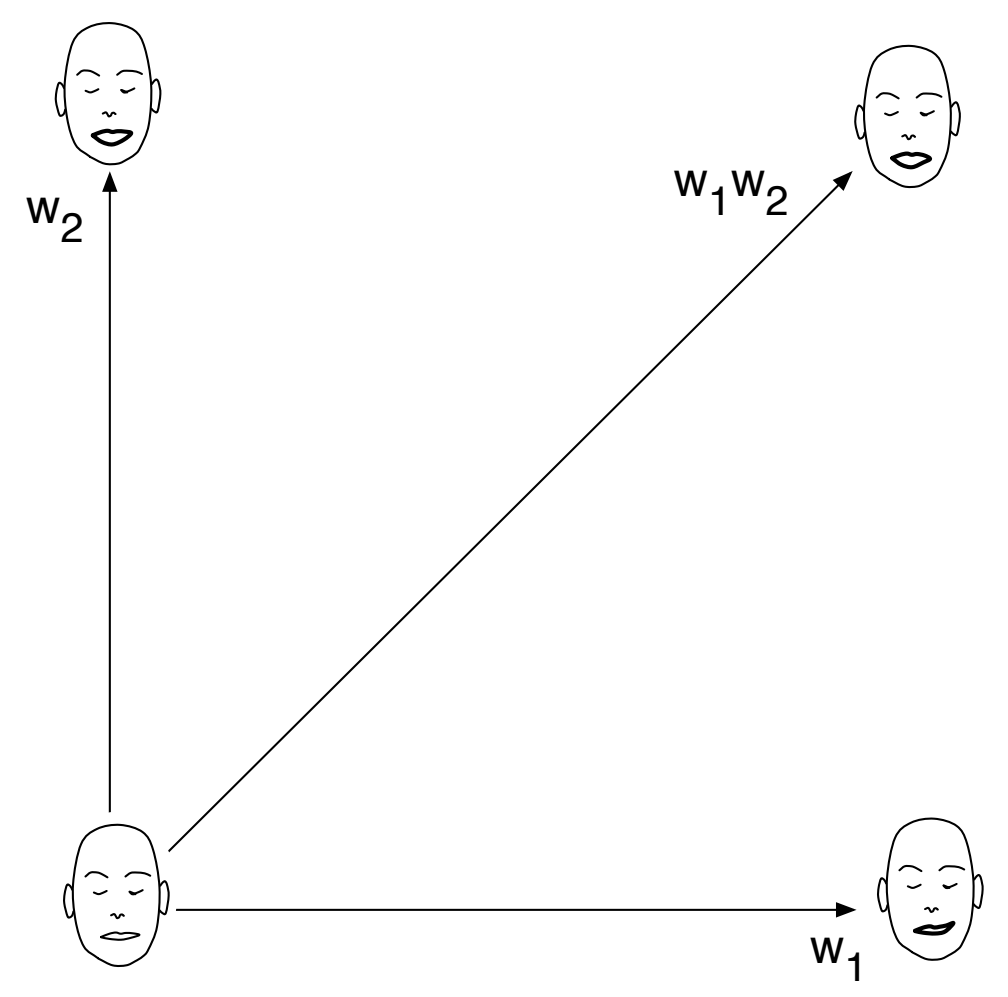

Figure 3.7: Schematic illustration of the "combination blendshape" idea [174]. A correction shape (top right) is added with weight $w_{1} \cdot w_{2}$.

be situated at intermediate weight values, giving piecewise linear interpolation. This is shown schematically in Figure 3.6.

\subsubsection{Combination blendshapes}

Another blendshape variant [174, 239, 266] adds additional "correction" shapes that become active to the extent that particular pairs (or triples, etc.) of weights are active. This scheme is variously called combination blendshapes or corrective shapes [266]. 


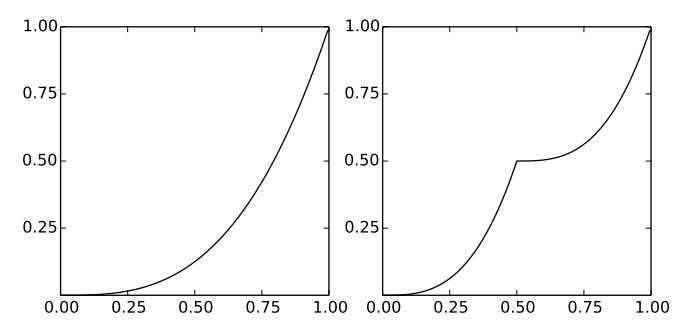

Figure 3.8: (Left) a third-order combination shape has weight $w_{i} \cdot w_{j} \cdot w_{k}$ for some triple $i, j, k$, and so has little effect until the weights approach one. (Right) When intermediate combination shapes are used, the resulting interpolation is not smooth.

This approach might be notated as

$$
\begin{aligned}
\mathbf{f}=\mathbf{f}_{0} & +w_{1} \mathbf{b}_{1}+w_{2} \mathbf{b}_{2}+w_{3} \mathbf{b}_{3}+\cdots \\
& +w_{1} w_{5} \mathbf{b}_{1,5}+w_{2} w_{13} \mathbf{b}_{2,13}+\cdots \\
& +w_{2} w_{3} w_{10} \mathbf{b}_{2,3,10}+\cdots
\end{aligned}
$$

Here the first line is equivalent to equation (3.4). A term $w_{1} w_{5} \mathbf{b}_{1,5}$ is a bilinear "correction" shape that is fully added only when $w_{1}$ and $w_{5}$ are both one, and is completely off if either is zero. The irregular numbering 1,5 is intended to indicate that these corrections are only needed for particular pairs (or triples, quadruples) of shapes such as shape 1 and shape 5. For example, the eyebrow and mouth corner are spatially well separated, so it is unlikely that any correction shape would be needed for this pair of shapes. A schematic visual representation of this approach is shown in Figure 3.7. The combination targets are situated at (some of) the diagonals of the blendshape hypercube.

The majority of the blendshape targets in modern professional models with hundreds of targets are these combination shapes. As an example, the primary targets (those situated at the hypercube vertices that are neighbors of the neutral shape) may number 100 shapes, whereas the number of combination shapes may be several hundred or more [197]. The com- 
bination blendshape idea should be distinguished from the on-line "correction" shapes that have been a subject of recent research (section 3.4.3). Correction shapes modify or add to the linear blendshape basis, whereas combination shapes can be seen as a second-order term in a Taylor series in the blendshape weights (section 3.6.4).

The combination blendshape scheme is not ideal from an interpolation point of view. When the facial expression travels along the (hyper)diagonal toward a 2nd order correction, the correction appears quadratically as $w_{i} w_{j} \mathbf{b}_{i, j, \ldots}$. Subjectively, this means that the correction has little effect over most of the range of the sliders and then appears relatively suddenly. The problem is exacerbated with 3rd and higher order corrections. This problem can be partially addressed by placing intermediate shapes along the diagonal.

\subsubsection{Hybrid rigs}

In a blendshape model the jaw and neck are sometimes handled by alternate approaches. For example, since the motion of the jaw has a clear rotational component, the jaw-open target is often augmented by linear blend skinning [173]. The eyelids are another area that is sometimes handled by alternate rigging approaches, again due to the rotational motion. Refer to [173] for a recent survey of facial rigging techniques.

\subsection{Constructing Blendshapes}

There are several approaches for creating blendshapes. A skilled digital artist can deform a base mesh into the different shapes needed to cover the desired range of expressions. Alternatively, the blend shapes can be directly scanned from a real actor or a sculpted model. A single template model can be registered to each scan in order to obtain vertex-wise correspondences across the blendshape targets. Methods to register scans (and 
register a generic template to scans) include [124, 4, 208, 252, 3, 7].

In concept, a dynamic mesh obtained from dense motion capture can be decomposed into a linear model using principal component analysis (PCA) or other approaches. (Refer to appendix A.6 for an introduction to PCA.) However, the PCA models lack the interpretability of blendshapes. This will be discussed further in sections 3.4.2 and 3.7.7.

In [188] blendshape targets are rapidly constructed with minimal manual assistance from multiple pictures of an actor. [33] fits a morphable model (PCA model of both the geometry and texture) to a single image, resulting in an estimate of the geometry and texture of the person's face. Typically the geometry of a facial model is fairly coarse, with fine scale details such as wrinkles and freckles represented via textures, bump or normal maps, or recent techniques such as [149, 29, 25]. In the case of bump or normal maps the decomposition makes good use of graphics hardware, and the choice of relatively coarse geometry in facial model capture and tracking applications can also be motivated from bias-variance considerations in model fitting [100].

\subsubsection{Model transfer}

Blendshape models can also be constructed by transferring the expressions from an existing source model to a target model with different proportions. Section 3.5.3 describes "expression cloning" algorithms for transferring the motion from one model to another. This subsection describes the related problem of constructing a target model that in some sense is equivalent to a given source model, given only a limited sample of the target expressions such as the neutral face. We term this problem model transfer. Note that model transfer algorithms can be used for transferring motion as well, simply by applying them to a moving source model. However not all expression cloning algorithms are suitable for model transfer.

Deformation transfer [226] is the leading approach for constructing a 
target model by model transfer. It requires a fully constructed blendshape model for the source, but only a neutral model for the target. This approach first finds the deformation gradient between each triangle of the neutral pose source model $\mathbf{b}_{0}$ and the corresponding triangle in one of the source blendshape expressions $\mathbf{b}_{k}, k \geq 1$ (The "deformation gradient" is the Jacobian of the function that deforms the source triangle from its neutral position). Then, given a non-neutral expression on the source model, deformation transfer finds triangles on a target expression so that the target deformation gradient matches the equivalent Jacobian on the source model in a least squares sense. [40] points out that deformation transfer is a form of Poisson equation.

Since the original deformation transfer does not consider collisions, it may result in self-collisions particularly around the eyelids and lips. [205] inserted virtual triangles into the eye and mouth openings of the mesh to prevent this problem. They also add a new term to the optimization that causes the Laplacian of target mesh to resemble that of the source in areas that are most compressed or stretched, reducing a tendency to crumple in these areas.

[141] is a technique designed specifically for the blendshape model transfer problem. This approach allows the artist to guide the model transfer process by specifying a small number of example expressions and corresponding approximate expected blendshape weights for these expressions. Since only a small number of example expressions are provided, construction of the full target basis is an underdetermined problem. This is solved by using the deformation transfer of the source as a regularization energy in their optimization.

\subsubsection{Discovery of blendshapes}

Creating a realistic blendshape model may require sculpting on the order of 100 blendshape targets, and many more shapes if the combination 
shapes scheme is used (section 3.3.3). Each target must be designed to capture its intended role such as approximating the activation of a particular muscle, while simultaneously minimizing undesirable interactions with other shapes. This is a labor intensive and iterative effort.

It would be ideal if one could start with dense motion capture of a sufficiently varied performance, and then automatically or semi-automatically convert this into a blendshape model. In abstract this is a matrix factorization problem

$$
\mathrm{M} \approx \mathrm{BW}
$$

where for a motion capture performance with $t$ frames and $p=m / 3$ vertices, the $m \times t$ performance matrix $\mathbf{M}$ is split into the $m \times n$ blendshape basis $\mathbf{B}$ and the $n \times t$ animation weights matrix $\mathbf{W}$. Typically the number of frames $t$ is larger than the number of basis vectors $n$, so this is a low-rank factorization. Doing PCA on the dense motion capture might be a first step toward this goal, however as pointed out elsewhere, the PCA basis vectors are global and lack the necessary semantics. Given a PCA model $\mathbf{f}=\mathbf{U} \mathbf{c}+\mathbf{m}$ with $\mathbf{U}$ being the eigenvectors and $\mathbf{m}$ the mean shape, the discovery problem can be formulated as finding a "recombination" matrix $\mathbf{R}$ such that the new basis UR in an equivalent model

$$
\mathbf{f}=(\mathbf{U R})\left(\mathbf{R}^{-1} \mathbf{c}\right)+\mathbf{n}
$$

is more sparse [134].

[169] addresses this blendshape discovery problem by minimizing $\| \mathbf{M}-$ $\mathbf{B W} \|^{2}$ subject to a sparsity penalty on the basis $\mathbf{B}$, where $\mathbf{M}$ is the sequence of scans or motion capture of the face, and $\mathbf{W}$ are the corresponding (unknown) weights. Rather than minimizing $\|\mathbf{B}\|_{1}$ to promote sparsity, they use an $\ell_{1}$ norm over the length ( $\ell_{2}$ norm) of each vertex. In other words, each vertex in the basis is encouraged to be zero, but if the vertex is not zero then there is no further penalization of its components [13]. The results outperform PCA, ICA, and several other algorithms and allow intuitive direct manipulation editing. 
While [169] is a significant advance, further developments may be possible on this important problem. It is likely that artists will prefer to guide the blendshape construction rather than relying on a fully automatic process, so an ideal solution must accelerate the artist's process without taking away control.

\subsubsection{Blendshape refinement}

Often a blendshape model will not exactly match the desired motion. One variant of this problem is when the motion causes the model to take on an expression that reveals undesirable interactions between the blendshape targets. In this case artists can resculpt the model or add corrective combination shapes as discussed in section 3.3.3. A second form of the problem is when the model matches the motion in a least squares sense but with a large residual.

To handle this case [110] fit the residual with a radial basis function scattered interpolation. [61] used a coordinate descent optimization to solve for positions of the basis vertices corresponding to the markers. This correction was then applied to the remaining vertices using radial basis interpolation. [114] addresses the refinement problem by augmenting the basis with new targets for frames with high residuals. The correction uses (bi)harmonic interpolation [28] of the tracked displacements.

While some of the previous methods optimize over all frames in a sequence, a focus of recent research is methods that can accomplish on-line refinement of the blendshape basis. Since the data in online methods is often of low quality, a key issue is to distinguish geometry from noise, and to decide when to stop adapting the basis. [142] address this problem using a color-depth video stream. The initial blendshapes of the actor's face are created using deformation transfer. Then, additional corrective PCA shapes refine the actor-specific expressions on the fly using incremental PCA based learning. [27] presents a system to refine animation 
curves and produce additional correctives from a set of blendshapes along with 2D features such as markers on the face and contours around eyelids and lips. Every frame is optimized using 2D marker constraints, 3D bundle constraints, and contour constraints. [41] combine a PCA model of identity with a blendshape model of expressions obtained through deformation transfer from a generic template model. Since a particular person's expressions are not exactly captured in this basis, they add a correction in the form of the low-frequency eigenvectors of the graph Laplacian of the face mesh. This correction basis can fit a smooth residual from the blendshape basis while rejecting the noise from the RGB-D camera used in their system.

\subsubsection{Detail enhancement}

An existing prior database of high-resolution facial poses can be used to enhance a low-resolution or noisy captured performance. The highresolution basis can be filtered to produce a corresponding low-resolution basis, which is then used to represent the low-resolution performance. The weights on this basis are then applied to the high-resolution basis, resulting in a face having the original performance but more detail. [25] develops and extends this idea, including temporal enhancement. Deformation gradients are used as the basis, so strictly speaking this approach is more general than blendshapes.

\subsubsection{Generating new models by interpolating in an exist- ing population}

We informally refer to the set of mesh vertices and edges as a topology. Given an existing set of blendshape models with common topology, it is possible to create new faces as weighted combinations of the existing models. This may be suitable for producing background or "crowd" characters. This approach is somewhat limited however: consider the case of 
two models, one of which has big eyes and a small mouth, and a second which has small eyes and a big mouth. Using a global linear combination of these two models, it is not possible to produce a new face with small eyes and a small mouth. A further issue is that (due to the central limit theorem) blends of a number of faces will tend towards a Gaussian distribution and will have less distinct features than the original basis faces. Allowing different linear combinations in different regions of the face is an obvious approach to increasing the diversity of the generated faces. This generates a new problem, however, in that the constructed regions will have discontinuities at their boundaries. Blending between regions is a poor solution, in that it is not obvious what the transition region should be. More importantly, a feature that is simply copied and blended onto another face is often not in the right location - for example, the mouth on some people is lower or higher (or further forward or back) than on others. [148] solved these issues by blending in the gradient domain (thereby providing continuity) and solving a Poisson problem to generate the composite face.

\subsubsection{Compressing blendshapes}

While the blendshape representation provides compression of an animation, further compression is desirable for animation editing, and is required for games. As an example for discussion, a blendshape model with 1000 targets, each with 10000 vertices represented with four-byte floats, would require 120 megabytes of memory. In the delta blendshape form most targets are localized and are zero at most vertices, so this size can be reduced using a suitable sparse matrix data structure.

While these figures indicate that a reasonably detailed model is easily accommodated in the memory of current processors, there are two reasons for needing additional compression. First, it is desirable (and required in games) that the scene includes the character body and background com- 
plete with textures. As well, many scenes have multiple characters. A more important reason is that the matrix-vector multiply $\mathrm{Bw}$ in equation (3.2) is memory-bound on both current CPUs and GPUs.

The obvious approach to compressing a blendshape model is to apply principal component analysis, retaining only the eigenvectors corresponding to the largest eigenvalues. As a rule of thumb, PCA can provide 10:1 or greater compression of many natural signals with little visible change in the signal. PCA does not work as well for blendshape models, however, because blendshapes are already a compressed representation - an animation of any length requires storage of only the $n$ basis vectors, and $n$ weights per frame. In several tests on available models, [212] found that the compression rates obtainable using PCA without introducing visible degradation are as small as 3:1. Another issue that is frequently overlooked is that the PCA coefficients are dense (Figure 3.15), which may result in reduced performance relative to blendshapes!

While blendshape models are resistant to PCA compression, they nevertheless have considerable structure and smoothness that can be exploited. [212] observe that it is possible to re-order the blendshape matrix $\mathbf{B}$ to expose large low-rank blocks. Placing these in "off diagonal" positions allows application of hierarchically semi-separable (HSS) algorithms [254]. These approaches produce a hierarchical compressed representation by compressing off-diagonal blocks and then recursively processing the diagonal blocks, and they provide a fast and parallelizable matrix-vector multiplication. Using a HSS representation [212] obtained on the order of 10:1 compression and similar speed increases.

\subsubsection{Outlook}

Approaches to constructing blendshapes have developed considerably in the last decade, including custom algorithms for face capture [17], the introduction of model transfer algorithms, algorithms for localized basis 
discovery, and tracking algorithms that provide on-line refinement of the blendshape basis. The current state of the art should easily permit automated capture of a blendshape-like facial basis suitable for automated tracking. There may be room for future methods that incorporate human guidance in the construction process and provide a basis that is closer to the muscle- or expression-based blendshape bases described in industry forums [174, 90, 101].

\subsection{Animation and Interaction Techniques}

Animating with blendshape requires specifying weights for each frame in the animation. For our discussion, animation techniques will be broadly divided into performance-driven animation techniques, keyframe animation, and direct manipulation. Performance-driven animation is commonly used to animate characters different from the actor, so expression cloning techniques will also be surveyed here. The section will conclude with a brief survey of alternative editing techniques.

\subsubsection{Keyframe animation}

Blendshape models have traditionally been animated using keyframe animation of the weights (sliders). Commercial packages such as Maya provide spline interpolation of the weights and allow the tangents to be set at keyframes. As an approximate figure, professional animation requires a keyframe roughly every three frames. Many animators prefer that keyframes include keys for all targets, rather than putting keys on each curve independently.

\subsubsection{Performance-driven animation}

In performance-driven facial animation, the motion of a human actor is used to drive the face model [250, 231, 64, 34, 190, 189, 247, 93]. Whereas 
keyframe animation is commonly used in animated films with stylized characters, performance-driven animation is commonly used for visualeffects movies in which the computer graphics characters interact with filmed characters and backgrounds. Because blendshapes are the common choice for realistic facial models, blendshapes and performance-driven animation are frequently used together.

The general literature on face tracking in general spans several decades and a complete survey is beyond the scope of this report. We will concentrate on performance capture methods that drive a blendshape rig. Techniques that drive a low-level representation such as a mesh will not be surveyed [250, 99, 253, 38, 43, 91, 19]. Methods that involve a nonlinear or physical underlying model are also not considered [231, 71, 219].

Performance capture methods might be classified into those that use 3D motion capture information as input [61, 73] versus methods that do model-based tracking of video [192, 34, 55, 200, 27, 49, 48]. Another distinction is whether a PCA basis [34] or blendshape basis [192, 60, 61, 64] is used. [73] uses a PCA basis for the motion capture which is then retargeted to a blendshape basis through a nonlinear radial basis mapping. [229] uses overlapping local PCA models.

Model-based tracking of blendshapes solves for the blendshape weights at each frame so as to match a reference video. Recent research has achieved high quality tracking from monocular video [93]. Typically the weights are constrained to the range $0 . .1$. When the source motion to match is available in the form of 3D motion capture, this is a constrained linear problem that can be solved with quadratic programming [60, 61, 110]. When model-based tracking is used to match images from a video, the perspective nonlinearity requires the use of nonlinear optimization (unless weak perspective is employed). [192] allowed soft constraints with a LevenbergMarquardt algorithm.

With the popularity and affordability of low-cost commercial depth cameras (e.g., Microsoft's Kinect), researchers have developed a number 
of techniques to utilize such cameras for performance driven facial animation. One approach does real-time tracking and transfers the facial movement to a user-specific blendshape face model that is manually constructed at the offline stage [246]. Recent advances include online modeling of user-specific blendshape faces (without the offline step) and introduction of adaptive corrective shapes at runtime for high-fidelity performance driven facial animation applications [27, 142, 41]. These basis refinement approaches are briefly surveyed in section 3.4.3.

\subsubsection{Expression cloning}

In expression cloning techniques [171, 226], the motion from one facial model (the "source") is retargeted to drive a face (the "target") with significantly different proportions. Expression cloning is frequently the goal of performancedriven animation. For example, an adult actor may produce the motion for a younger or older person (as in the movies The Polar Express and The Curious Case of Benjamin Button) or a non-human creature (as in Avatar and the Gollum character in the Lord of the Rings movies). A very similar problem is that of creating a full target face model, given the source face but only limited samples of the target, usually only the neutral shape. This problem discussed in section 3.4.1. Algorithms such as [226] mentioned in that section can also be used for expression cloning.

[171] introduced the expression cloning problem. Their approach requires only a generic animated facial mesh for the source and makes no assumption of a blendshape or other representation. It establishes a mapping by finding corresponding pairs of points on the source and target models using face-specific heuristics. [240, 70] discover a tensor basis that spans both expression and identity in different dimensions. Identity can be flexibly manipulated in this approach, however it does not use a blendshape basis.

A common approach to expression cloning is what might be termed 
"corresponding parameterization" [62, 106, 190, 141]: source and target blendshape models are constructed to have the same number of targets, with the same semantic function (typically FACS inspired). The blendshape weights are then simply copied from source to target. This approach is simple, and allows great flexibility in developing the cross-mapping. For example, one could imagine a smi le blendshape for a lizard character in which the mouth corners move backward whereas in the corresponding blendshape for the human the mouth corners are displaced upward. Expression cloning using corresponding parameterization based on FACS expressions [81] was introduced in the movie industry on projects such as Monster House and King Kong [90].

The corresponding parameterization approach requires artists to construct blendshape models for both the source and target faces. In the usual case where the source is obtained by performance-driven animation, this can be avoided by requiring the actor to produce the set of basis expressions, for example by mimicking FACS expressions. Producing expressions consisting of individual facial muscles is an unnatural and difficult task for many people, however. In fact it is believed that some facial muscles can only be activated indirectly, as a side effect of producing other expressions, but not under voluntary control [80].

Alternately, the source basis can be obtained by taking expressions from an actual performance. Chuang and Bregler [63] experimented with several principles for choosing frames from the performance, finding that the best approach selects expressions that have maximal projection on the leading principal components of the source performance. Specifically, the first two basis shapes are those that have the largest and smallest projection on the leading eigenvector, the second two have the maximum and minimum coefficients on the second axis, and so on. The artist then poses the target model to correspond to each of the chosen basis vectors. Note that while this algorithm involves PCA, the actual basis shapes are expressions from the performance rather than eigenvectors, so creating a corre- 
sponding pose on the target model is a natural task. [63] also found that requiring the weights to be positive produced better cloning than allowing negative weights, even though the resulting reconstruction of the source animation has higher error. Intuitively, allowing negative weights allows the basis to "explain" small details of the source motion using a variety of unintended cancelling (positive and negative) combinations of the basis shapes, which has poor results when the same weights are applied on the target model. This intuition is related to the interpretation of non-negative matrix factorization as a parts-based decomposition [125].

More generally, if the source and target models already exist, but do not share a parameterization, it may be possible to learn a cloning function given sufficient examples of corresponding poses. In a linear version of this idea, there need to be $c \geq n$ corresponding poses if the models contain $n$ blendshape targets. Let $\mathbf{w}_{k}$ be the blendshape weights for the source, and $\mathbf{v}_{k}$ be the blendshape weights for the target, for each pair $k$ of corresponding poses. Gather $\mathbf{w}_{k}$ and $\mathbf{v}_{k}$ as the columns of matrices $\mathbf{W}, \mathbf{V}$ of dimension $n \times c$. Then an expression cloning matrix $\mathbf{E}$ of dimension $n \times n$ that maps $\mathbf{w}$ to $\mathbf{v}$ can be found,

$$
\begin{aligned}
& \mathbf{W}=\mathbf{E V} \\
& \mathbf{W} \mathbf{V}^{T}=\mathbf{E} \mathbf{V} \mathbf{V}^{T} \\
& \mathbf{E}=\mathbf{W} \mathbf{V}^{T}\left(\mathbf{V} \mathbf{V}^{T}\right)^{-1}
\end{aligned}
$$

This simple linear expression cloning approach has its limitations - in particular in that the mapping is linear (as is the case with some other approaches including corresponding parameterization).

Still more generally, in what might be termed semantic correspondence the source and target representations need only agree on a set of parameters that control the expression, with each having some mapping or algorithm to convert between these parameters and the internal representation of the model. This general approach may have been first demonstrated by SimGraphics in the 1990s [251]. 
Most existing expression cloning algorithms do not consider adapting the temporal dynamics of the motion to the target character, and instead assume that if each individual frame can be transferred correctly, the resulting motion will be correct. This will tend to be the case if the source and target are of similar proportions.

There are several scenarios in which the temporal dynamics of face movement should be considered however. One case is where the target cannot reproduce the full range of movement of the source model. For example, the target jaw may not open widely enough to reproduce the source motion. These limits commonly occur when a blendshape model is driven directly by motion capture. They also can occur even when the source is a blendshape model. For example, the target model might allow jaw-open to range up to 1 , but it may be that the results look unnatural if smile is simultaneously active with a value of more than 0.7 . This situation can be crudely handled with an expression that sets the limit on the jaw-open as jaw-open-limit $=1-0.3 *$ smile.

In this situation, [214] argue that reproducing the source on a per-frame basis results in unnatural motion when the target motion limit is reached and exceeded. They propose that it is better to preserve the overall shape of the motion, rather than matching the position of each frame independently. This objective is formulated by saying that the temporal derivatives (rather than positions) should be matched in a least squares sense. This leads to a space-time Poisson equation that is solved for the target blendshape motion.

More generally, most current expression cloning techniques require that the target expression for a particular frame be a function of the source expression for that frame only. More powerful expression cloning techniques may require looking at adjacent frames in order to allow anticipation and coarticulation-like effects to be produced.

An open problem is the case in which the target motion should differ from that of the source is when the target has significantly different pro- 


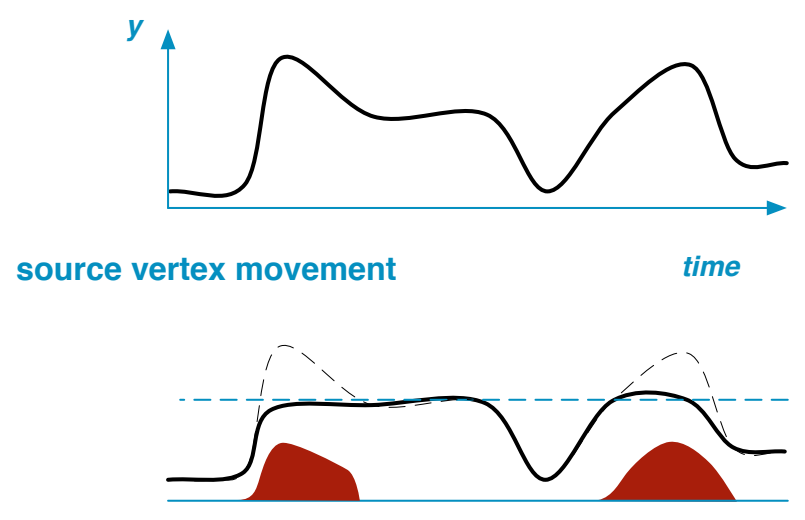

target movement, position domain transfer

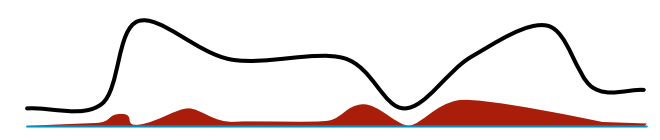

target movement, gradient domain transfer

Figure 3.9: The "movement matching" principle in [214]. The target cannot fully reproduce source movement (top) due to limitations of the target geometry. Attempting to best reproduce the position of each frame results in clipping when the pose is not achievable (dashed line in middle figure). Instead, the movement matching principle attempts to match the temporal derivatives, thereby reproducing the shape of the motion (bottom). The red shaded areas indicate the magnitude and distribution of the matching error. 
portions or size from the source. The human mouth moves very quickly during speech - for example the mouth can change from a fully open to a fully closed position in two adjacent video frames. Transferring this rapid motion to a large and non-humanoid character such as a whale would likely give implausible looking results.

On the other hand, we recall the anthropomorphic principal that the target character is usually humanoid if not human - if the character needs to be perceived by human audiences, it needs to express facial emotion in human-like ways. Thus, it is not clear if very significant deviations from human-like (temporal) performance are likely to be useful.

\subsubsection{Stabilization}

Retargeting of motion capture requires determining the coordinate frame of the skull. The motion of this frame is removed, and the remaining motion of the face determines the facial expression. The rigid coordinate frame of the skull is not easily determined, however, and if it is poorly estimated subsequent analysis may conflate head motion with expression change. The issue is that people cannot naturally produce expressions without simultaneously moving the head. One approach to this problem is to attempt to find at least three relatively stationary points on the face, and estimate the rigid transform from these - typical candidates are the corners of the eyes and the nose tip. However, some people slightly move these points (relative to the skull) while making extreme expressions. Another solution is to identify the head motion using a rigid hat. However vigorous movement or particular expressions (such as raising the eyebrows strongly) may cause the hat to move slightly. Facial expressions can be very subtle (consider the geometric difference between a face expressing the two emotions "calm" and "contempt").

[18] introduced an approach to this important problem. It first deforms a generic skull model (including a nose) to fit manually specified land- 
marks on a neutral pose of the actor. Then, the rigid position of the skull relative to a new scan of the face surface is determined by optimizing a cost involving the expected distance (thickness) between the skin and the skull and a second cost involving the nose length. The results are validated by comparison of the stabilized upper teeth to those in reference images. An earlier approach [258, 55] treats the problem of separating rigid motion from deformation as a matrix factorization problem. That approach requires that the face position is described by a set of 3D tracking markers, i.e. the correspondence problem is solved, and the tracked points are a discrete set of markers rather than dense motion capture.

\subsubsection{Partially-automated animation}

In practice, performance-driven animation is rarely used without subsequent manual adjustment. One reason for this is lack of fidelity or errors in the motion capture process. For example, marker-based systems typically place markers around the outside of the mouth are thus not able to track the inner contour of the lips ([27] is a recent exception). Similarly, most motion capture systems do not track the eyes or eyelids.

There is another important reason for editing performance-driven animation: changes in the acting may be required. This may be because a performance that is automatically transferred to a different (e.g. non-human) character may not convey the intended emotion. As well, a movie director can request changes in the performance. For these reasons, a viable performance-capture system must allow for subsequent manual editing by artists. This is a major reason why existing performance capture approaches use a blendshape representation.

Subsequent editing of motion capture presents a further problem: motion capture produces weight curves with a key at every frame. This is too "dense" for artists to easily edit. [215, 130] introduced an optimal curve simplification technique using dynamic programming. With a GPU im- 
plementation, it can produce roughly an $80 \%$ reduction in sample density with little or no visible difference in the resulting curve.

\subsubsection{Direct manipulation}

Blendshapes have traditionally been animated with keyframe animation or by motion capture. Although inverse kinematics approaches to posing human figures have been used in animation for several decades, analogous inverse or direct manipulation approaches for posing faces and setting keyframes have appeared only recently. In these approaches, rather than editing the underlying parameters (as in forward kinematics, and keyframe animation), the artist directly moves points on the face surface and the software must solve for the underlying weights or parameters that best reproduce that expression or motion.

The evident challenge for direct manipulation of faces is that it can be a very under-constrained inverse problem - similar to inverse kinematics, but more so. In moving the hand of the character using inverse kinematics, for example, the animator specifies a goal point (3 degrees of freedom), and animation system must solve for on the order of 10 degrees of freedom representing the joint angles from the hand through the shoulder. In a professional blendshape model, the analogous number of unknown weights can be 100 or more. Solving the inverse problem for direct manipulation blendshapes then means that we find a discrete function (i.e., a vector $\Delta \mathbf{w}$ ) that satisfies the constraint given by a pin-and-drag manipulation [259] of a 3D face model. The resultant weights are then (usually automatically) interpolated to make a whole animation. The central issue here is the choice of a strong and appropriate prior for regularizing the inverse problem.

It is important to note that professional animation requires providing both direct manipulation and access to the underlying parameters (sliders). Intuitively, this is because some edits are simply harder to accom- 
plish using direct manipulation. In fact it is easy to argue on mathematical grounds that slider manipulation is necessarily more efficient for some edits, whereas the converse is also true - direct manipulation is necessarily more efficient for other edits. Briefly, this is because of the spreading effect of a multiplication by a non-identity matrix [131]. In direct manipulation the blendshape weights are in a pseudoinverse relationship to the manipulated points, and columns of the pseudoinverse tend to have a number of non-zero values.

\section{Direct manipulation of PCA models}

The underconstrained direct manipulation inverse problem was first solved by several approaches that use an underlying PCA representation. [261] develop a hierarchical segmented PCA model. User-directed movement of a particular point on the face is propagated to the rest of the face by projecting the altered point vector into the PCA subspace and iterating this procedure over the remainder of the hierarchy. [159] learn a PCA subspace of facial poses. This is used to bypass computation of a majority of face points, by "PCA imputation" wherein a subset of points is computed and fit and the same linear combination is used to estimate the locations of the remaining points. [144] use a local, hierarchical PCA face model; facial editing is performed with a constrained version of weight propagation [261]. This provides local control while also allowing natural cross-region correlations. [122] develop direct dragging and stroke-based expression editing on a PCA model obtained from motion capture data, and include a statistical prior on the space of poses.

These PCA approaches are good solutions if the model will be manipulated exclusively with direct manipulation, and this is the most appropriate choice for novice users. Since professional animation also requires access to the underlying sliders however, this in turn necessitates the use of an underlying blendshape representation rather than PCA due to the lack of interpretability of the PCA basis (section 3.7.7). While it is easy to 


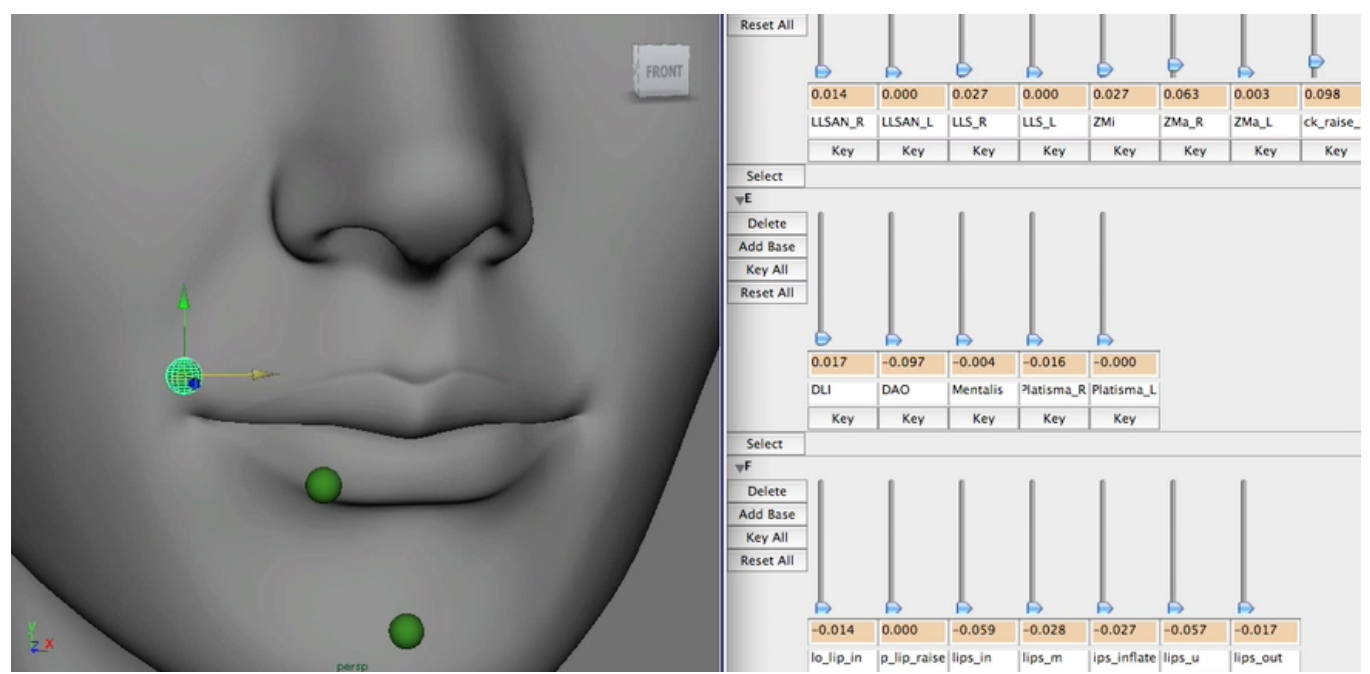

Figure 3.10: Screenshot of a direct manipulation interface in operation. (Left panel) selecting a point on the model surface creates a manipulator object termed a pin. These can be dragged into desired positions, and the system solves for the slider values (right panel) that cause the face to best match the pinned positions.

interconvert between PCA and blendshape models (section 3.7.8), doing so requires having a blendshape model.

\section{Direct manipulation of blendshapes}

[260] included a direct manipulation algorithm in their facial capture system. It used a basis of selected frames from a captured performance, and allows direct face editing using local and adaptive radial basis blends of basis shapes. They introduced an interesting regularization for the direct manipulation inverse problem, in which the basis meshes most similar to the desired constraints are weighted more heavily. This is an effective approach to extending the span of a model with a limited number of shapes (see Figure $6(\mathrm{~d}),(\mathrm{e})$ in [260]), though with a more extensive model this property might be considered undesirable. 
The inverse problem can be avoided by using a fully constrained approach, exactly as would be used for performance driven animation. In this approach the artist interacts with manipulators that serve the same role as motion capture markers. The manipulators cover the face and are moved one at a time, with the others remaining stationary. The first published approach to direct manipulation of blendshape models [110] used this approach.

While constraining the face with a full set of manipulators avoids the inverse problem, it can also increase the required number of edits since no part of the face is free to move without intervention from the artist. Formulating direct manipulation as an underconstrained inverse problem allows many parts of the face to move during each edit, but requires a sensible regularization to make this useful (the previous fully constrained version of the problem can be recovered as a special case by adding sufficient constraints). [131] started with the principle that moving a particular part of the face should cause the remainder of the face to change as little as possible - a principle of "least surprise". To embody this in an algorithm, they observe that the blendshape model itself is designed as a semantic parameterization, that is, the targets are sculpted so that facial expressions can be described by the combination of $n$ sliders, each with approximately equal effect on the facial expression. This is in contrast to PCA, where the subsequent coefficients by definition have smaller influence. Thus the change in facial expression is to a first approximation represented by the change in weights, as demonstrated in Figure 3.1. In this figure Euclidean distance on the control vertices indicates that the full smile and open-mouth expressions are most similar, but the distance between the blendshape weight vectors correctly indicates that the smile is semantically and perceptually more similar to the half-smile.

[212] presents a direct manipulation system suitable for use in animation production, including treatment of combination blendshapes and non-blendshape deformers. They add an improved regularization term 
that better handles the common case where the artist repeatedly moves a single slider over the same range of values in order to understand its effect. The nonlinear components of their production-quality rig are handled with a combination of nonparametric regression (for the jaw) and a derivative free nonlinear optimizer. [11] describes an extension of the direct manipulation approach [131], which allows more efficient edits using a simple prior learned from facial motion capture. This system also allows the artist to select between three different modes at any time during editing: sliders, regular, and learned direct manipulation (see section 3.7.8. [53] describe an approach in which the artist designs direct manipulation manipulators by sketching. [169] show direct manipulation of an automatically created local linear model. This work is discussed in section 3.4.2.

\subsubsection{Further interaction techniques}

[188] proposes a painterly interface for creating facial expressions. The interface has three components: a canvas for designing a facial expression, a brush interface that let the user selects the intensity and decay of the strokes, and a palette where the colors have been replaced by facial expressions. When a stroke is applied to the facial canvas, weights from the selected facial expression are transferred and blended. When completed the facial canvas can be added to the facial palette and selected to design more complex expressions.

While direct manipulation offers advantages over the traditional slider editing, a more fluid or "sketch based" interface [161] might be preferable for both novice users and for previsualization of professional animation. Development of a sketch-based system that interoperates with an underlying blendshape basis is an open problem. 


\subsubsection{Outlook}

Methods for automated tracking, expression cloning, and interacting with blendshape models are well developed. Open areas may include expression cloning methods that consider differing characteristics of the target model, e.g. those resulting from considerable differences of anatomy or size. Another open area may be the development of interfaces (e.g. sketchbased interfaces) for faster and more fluid manual animation.

\subsection{Facial Animation as an Interpolation Prob- lem}

Blendshapes are perhaps the simplest approach to facial animation imaginable, and limitations of the linear model are evident. In this section we discuss blendshapes in abstract as a problem of interpolation, and consider whether a better approach may be possible.

\subsubsection{Blendshapes as a high dimensional interpolation prob- lem}

In abstract, facial animation is an interpolation problem of the form

$$
f: \mathbf{R}^{n} \rightarrow \mathbf{R}^{3 p}
$$

that maps a set of $n$ animation control parameters (such as $n \approx 100$ for blendshape sliders) to the $3 p$ values, where $p$ is the number of control vertices of the 3D face model.

\subsubsection{Linear interpolation}

The linear nature of blendshapes affects the animation in some cases. In the interpolation from one target to another, two weights change in a convex combination, and the movement of each changing vertex is necessarily 
along a line. Animators are aware of this limitation [228] and have sometimes compensated for it by adding additional sculpted shapes that are interpolated on the animation timeline. If the two weights are not in an affine (sum-to-one) combination, the movement is constrained to a plane, etc. More generally, the blendshape scheme constrains movement to a $n$ dimensional subspace of the $3 m$-dimensional ambient space.

\subsubsection{Scattered interpolation}

A scattered interpolation scheme might seem an ideal solution to the problem of interpolating a number of targets in a high dimensional space, since the sculpted faces could be placed at arbitrary (scattered) desired locations in the parameter space $\mathbf{w}$ (Figure 3.11). In a radial basis function (RBF) approach the kernel could be chosen as the Green's function of a differential operator, resulting in smooth interpolation of the data. This formulation would also separate the number of targets from the dimensionality of the space.

Unfortunately, high-dimensional interpolation is known to be intrinsically difficult [47, 92]. The Green's function corresponding to the differential operator family $\nabla^{2 s}$ is defined as [79, 137]

$$
R(\mathbf{x}) \propto \begin{cases}|\mathbf{x}|^{2 s-n} \log |\mathbf{x}| & \text { if } 2 s-n \text { is an even integer, } \\ |\mathbf{x}|^{2 s-n} & \text { otherwise }\end{cases}
$$

for smoothness order $s$ and space dimension $n$.

This requires a condition $2 s>n$ in order to avoid having a singularity at the origin. A potentially more difficult problem is the curse of dimensionality (appendix A.7; [100]), which suggests that the number of data samples required for interpolation in $n$ dimensions is exponential in $n$, unless the interpolation scheme can identify that the data lives on a lower-dimensional manifold or makes other simplifying assumptions.

Thus, we have the open problem of interpolating in a high (e.g. $n=$ 100) dimensional space. One possibility would be to dramatically increase 
the order of smoothness $s$, to $s>n / 2 \approx 50$. While this has not been explored, it can be noted that in other applications in computer graphics $\mathcal{C}^{2}$ smoothness has often proven sufficient, and at present we have no reason to believe that the motion of the face between expressions is extremely smooth.

\subsubsection{Blendshapes as a tangent space}

Equation 3.4 resembles a vector-valued Taylor series expansion about the neutral face, i.e.,

$$
f(\mathbf{w})=f(0)+\frac{\partial \mathbf{f}}{\partial \mathbf{w}} \cdot \mathbf{w}
$$

with $f(0) \equiv \mathbf{b}_{0}$ and the Jacobian $\left[\frac{\partial f_{i}}{\partial w_{j}}\right] \equiv \mathbf{B}$. In abstract geometric terms, we might consider blendshapes to be the tangent space (about the neutral face) of the $n$-dimensional face "manifold" embedded in a $m$-dimensional ambient space. As we move from one point to another along this (for example) 100-dimensional tangent space, the location in the $m=30000$ dimensional ambient space also changes.

This comparison to a Taylor series suggests limitations of the blendshape approach, and one wonders whether an alternative approach is possible. The blendshape approach requires the artist to sculpt $n$ shapes at all the locations in weight space $\mathbf{w}_{i}=\delta_{i, k}$ for $k=1 \ldots n$ (the vertices of the hypercube connected by an edge to the neutral shape, (Figure 3.5), i.e. the "one-ring" of the neutral). It is not possible for the artist to specify shapes at an arbitrary location such as $w=0.3,0.7,0.1, \cdots$ (Figure 3.11). If the facial model is incorrect at an arbitrary location, current systems require the artist to modify a number of targets so that their weighted sum reduces the desired correction, while simultaneously not disturbing other face poses. This is a time-consuming iterative refinement procedure.

[216] described a hybrid approach in which a basic blendshape model is augmented with additional nonlinear corrections. The corrections are interpolated by a radial basis function scheme inspired by weighted pose 


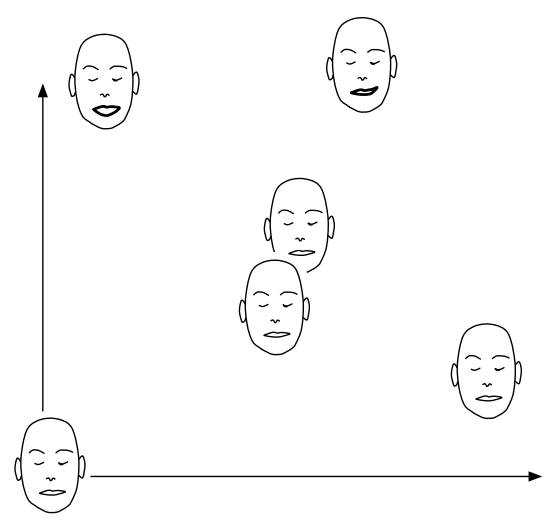

Figure 3.11: Blendshape schemes require that targets are placed at constrained locations, i.e. the vertices of a "weight hypercube" (Figures 3.5 . 3.7). It would be preferable to allow targets to be placed anywhere in face space, allowing the sculpting effort to be directed specifically where it is needed.

space deformation [120], with the underlying blendshape weights defining the pose space. This approach allows shapes to be placed as needed at any pose of the model (Figure 3.11) and the interpolation is smooth and free of artifacts such as the quadratic ramp-up that occurs with combination shapes (section 3.3.3).

\subsubsection{Outlook}

Interpolation in high dimensions is an open problem and an active subject of research in machine learning. Current approaches include additive models and (more generally) smoothing spline ANOVA models [241, 98], and approaches that make use of the manifold assumption. Interestingly, [120] can be seen as partially addressing the curse of dimensionality inherent in high dimensional interpolation, by breaking the global interpolation problem into a collection of softly coupled local problems. 


\subsection{The Blendshape Parameterization}

Despite the simplicity of the blendshape representation, there are a number of associated issues. The distinction between blendshapes and other linear models such as PCA is at the heart of the definition of blendshapes - indeed, otherwise there would be no need for a separate term. These issues will be surveyed in this section.

\subsubsection{Lack of orthogonality}

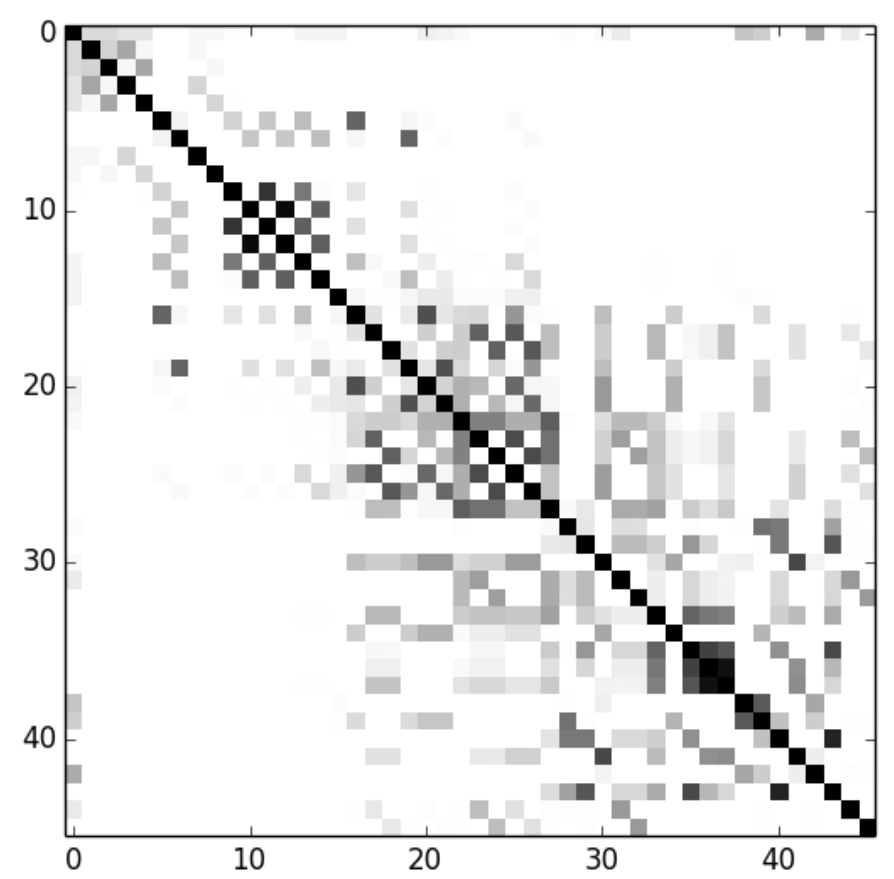

Figure 3.12: Mutual coherence plot for the 46-target blendshape model shown in Figure 3.10 and other figures. The $i, j$ entry is the covariance between the $i$-th and $j$-th blendshape targets, i.e. $\frac{\mathbf{b}_{i}^{T} \mathbf{b}_{j}}{\left\|\mathbf{b}_{i}\right\|\left\|\mathbf{b}_{j}\right\|}$. 
The major distinguishing characteristic of blendshapes relative to the more common principal component representation is that the shapes are not orthogonal (Figure 3.12). This has the advantage of interpretability (section 3.7.6). It has the disadvantage that the parameters are correlated, and so adjusting a parameter can degrade the effects obtained with previous edits. [136] addressed this problem with a user-interface technique in which the artist can "pin" particular points representing desirable aspects of the current facial expression, and subsequent edits occur in the approximate null-space of these constraints.

\subsubsection{Blendshape models are not unique}

Given a particular blendshape model, there are an infinite number of other blendshape models that can produce the same range of animation. Intuitively, this is similar to the fact that an infinite number of vector pairs span the plane, and given two such vectors (analogous to a particular "model"), another pair can be constructed as weighted combinations of the original vectors - for example the sum and difference of the original pair is one such basis. Given a particular blendshape model B, an arbitrary non-singular $n \times n$ matrix $\mathbf{R}$ and its inverse can be inserted between the $\mathbf{B}$ and the weights without changing anything:

$$
\mathbf{f}=\mathbf{B}\left(\mathbf{R R}^{-1}\right) \mathbf{w}
$$

Then $\mathbf{B R}$ is a new blendshape basis with corresponding weights $\mathbf{R}^{-1} \mathbf{w}$ that produces the same range of motion as $\mathbf{B}$.

\subsubsection{Equivalence of whole-face and delta blendshape for- mulations}

Proponents of various blendshape approaches are outspoken in industry forums regarding the proposed advantages of each particular approach. While working in the entertainment industry, the author heard emphatic 
claims that the delta form is the most powerful form of blendshape, or alternately that using targets modeled after the FACS poses [204, 81] is the only approach that produces all and only the full set of valid face shapes. In fact it is simple to show that, while these techniques have their respective advantages, they are equivalent in expressive power and the desired range of expressions does not uniquely specify a blendshape model.

The delta formulation equation (3.4) and the whole-face form equation (3.2) can be seen to be equivalent (in the terms of the range of shapes produced) by rewriting equation (3.1) as

$$
\begin{aligned}
\mathbf{f} & =\sum_{k=0}^{n} w_{k} \mathbf{b}_{k} \\
& =w_{0} \mathbf{b}_{0}+\sum_{k=1}^{n} w_{k} \mathbf{b}_{k} \\
& =w_{0} \mathbf{b}_{0}+\sum_{k=1}^{n} w_{k} \mathbf{b}_{k}-\sum_{k=1}^{n} w_{k} \mathbf{b}_{0}+\sum_{k=1}^{n} w_{k} \mathbf{b}_{0} \\
& =\left(\sum_{k=0}^{n} w_{k}\right) \mathbf{b}_{0}+\sum_{k=1}^{n} w_{k}\left(\mathbf{b}_{k}-\mathbf{b}_{0}\right)
\end{aligned}
$$

If the whole-face weights are convex (as is generally the case) this exactly recovers the delta-face formulation (3.3).

It is intuitive to think of local blendshapes as having more power for a given number of targets. For example, if there are $n_{1}$ shapes for the mouth and lower face, $n_{2}$ for the right eye and brow, and $n_{3}$ for the left eye and brow, then we may be tempted to consider that the resulting system would require $n_{1} \cdot n_{2} \cdot n_{3}$ whole-face shapes to have equivalent power. In fact this is incorrect, as suggested by equation (3.6) above. As an analogy, consider a pixel (sample) basis and a Fourier basis. The former is maximally local, yet spans the same space as the latter.

As an example, consider a blendshape model that has these targets: left-eye-closed, right-eye-closed (as well as the neutral shape). In the delta scheme, creating a model with both eyes closed requires corresponding 
weights $(1,1)$. In the whole-face scheme, setting the weights to $(1,1)$ would cause the head to scale, whereas setting them to $(0.5,0.5)$ will give a the result of two half-closed eyes. However if we notate the delta blendshapes as $b_{1}, b_{2}$, and the corresponding whole-face targets as $B_{1}=b_{1}+n, B_{2}=b_{2}+$ $n$, simple algebra gives the result that the desired closed-eye expression in delta form, $b_{1}+b_{2}+n$, is equivalent to $B_{1}+B_{2}-n$. Note that this is not a convex weight combination.

\subsubsection{Global versus local control}

In general, both global and local specification of shape deformation may be desirable. Global specification is desirable when the modeler is given a picture or sculpted maquette of a complete head that they must match with a computer model. Modeling a set of heads with various facial expressions is a more natural task than modeling the corresponding "delta" shapes such as the displacements governing eyebrow movement. Global specification is also used in some animation scenarios, such as the timedependent blendshape modeling approach mentioned in section 3.2 .

On the other hand, many animation tasks are more easily approached if local control is available. For example, increasing the width of the mouth is more easily accomplished if only one or a few blend shapes affect the mouth region than in the situation where every basis vector affects all regions of the face including the mouth. While producing the desired effect should be possible in an equivalent system of non-localized blendshapes (equation (3.6)), the global effect of each blendshape combined with their interaction with other shapes (see section 3.7.1) results in a tedious trial and error process for the artist. Fortunately, equation (3.6) points out that converting between whole-shape and delta formulations is a simple matter. Because of this equivalence and the simplicity of converting between the whole-face and delta formulations, it is not necessary to restrict oneself to the choice of one representation over the other - the user interface can 
allow the artist to select between the whole-face and delta forms according to the particular task.

As noted above, local control can be obtained with the delta blendshape formulation if the changes in the target faces are restricted to small areas. This may be difficult to obtain in some common modeling methodologies, however, as when the target faces are digitized from physical models. We also noted that local control can be guaranteed by segmenting the face into separate regions each of which has an independent set of blend shapes [115, 110]. Unfortunately the ideal segmentation may be difficult to choose in advance, particularly because designing blend shapes is a trial-and-error process, with many iterations of modeling corrections typically being required. [144] approaches the problem with a hierarchical (PCA) basis, thereby providing both local control and cross-region correlations. Automated creation of localized blendshapes is a goal of several research efforts [110, 73, 169]; these approaches are discussed elsewhere in this chapter.

\subsubsection{Convex combination of shapes}

Whole-face blendshape interpolation can be restricted to convex combinations by enforcing the following constraints on the weights

$$
\begin{array}{ll}
\sum_{k=1}^{n} w_{k} & =1 \\
w_{k} & \geq 0, \quad \text { for all } k .
\end{array}
$$

These constraints guarantee that the blendshape model lies within the convex hull of the blendshapes. This is a reasonable first assumption, but it is desirable to relax it. By analogy with the convex hull containing a two-dimensional face space (Figure 3.13), it is likely that targets sufficient to span a broad range of facial expressions must themselves lie outside the valid range of expressions. Because it is somewhat unnatural to ask an artist to sculpt targets that are slightly beyond the range of plausible 


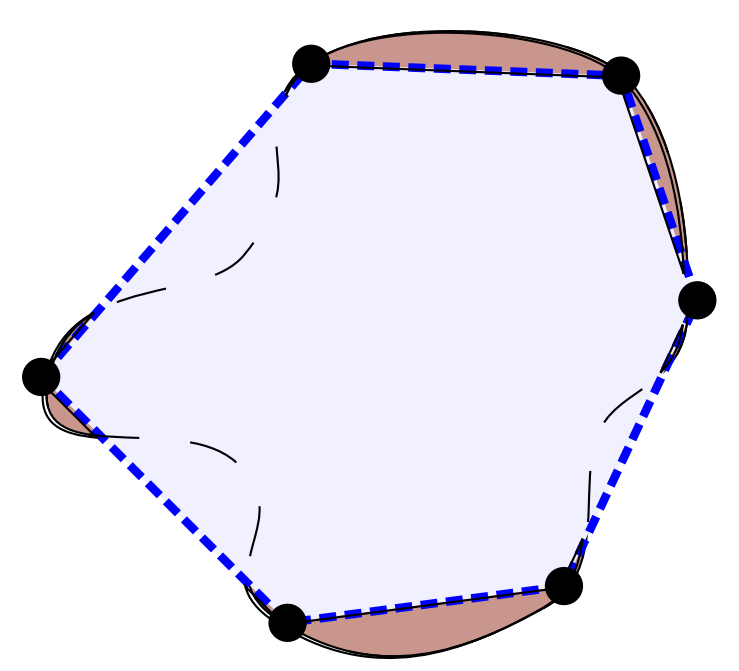

Figure 3.13: The space of valid face shapes, represented abstractly as the curved shaded region, is approximated as a convex combination of a number of blendshapes lying on the boundary of the region (black circles). Some regions of the space are not reachable with these blendshapes. This can only be addressed by sculpting blendshapes that lie outside of the valid face space. This is an unnatural task for the modeller.

expressions, it is often desirable to slightly relax the constraint in equation (3.7).

Constraining the weights to sum-to-one results in an inconvenient parameterization in which the model has $n$ user parameters for $n-1$ degrees of freedom, and any weight can be expressed as a linear combination of the other weights. In practice it means that the blending weights cannot be modified independently (e.g. using sliders) without violating the constraint. One solution is to normalize the weights after each modification. From the user interface point of view, this has the undesirable consequence 


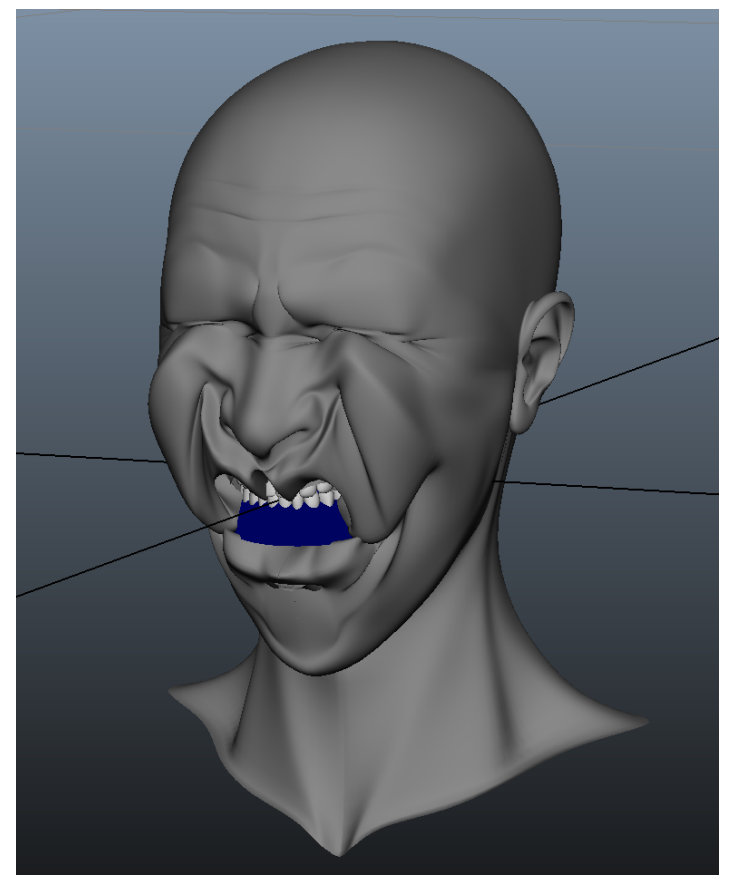

Figure 3.14: Blendshapes appear to function as a sparse basis. This figure shows a professionally created model with 45 targets, all set to one. Combinations of several (perhaps up to five or so) targets produce useful expressions, but the combination of many targets produces unusable shapes.

that changing a particular weight will cause other weights that were not explicitly altered to change as well. Animators are not novice computer users, however, and can learn to anticipate this behavior.

\subsubsection{Semantic parameterization}

The blendshape basis has meaning by construction: blendshape targets have simple and definable functions such as raise-right-eyebrow. This allows the effect of particular targets to be predicted and remembered, thereby reducing trial-and-error exploration during animation.

Recent literature in several fields explores the idea that sparse, positive, 

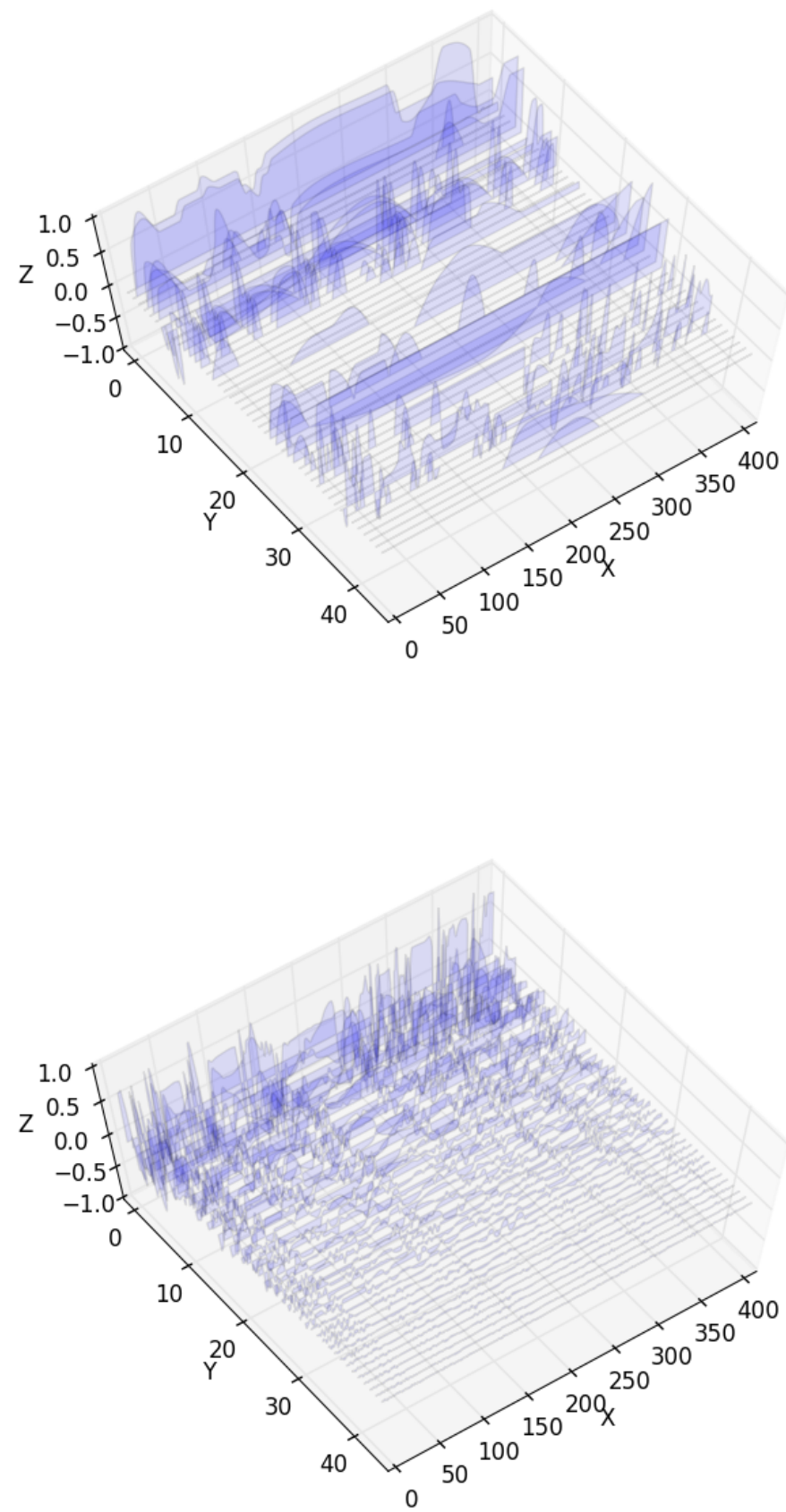

Figure 3.15: Comparison of blendshape (top) and PCA coefficients encoding (bottom) of the same 405-frame animation (X-axis) of a 45-dimensional (Y-axis) professionally authored face model. The blendshape coefficients are visibly sparser. 
non-orthogonal, and redundant bases are better able to encode aspects of the meaning of a signal. Examples of this literature include non-negative matrix factorization [125], sparse coding for image processing [82], and modeling of biological information processing [172].

We note that blendshapes share the qualities of being a non-orthogonal and sparse representation. The blendshape weights are (usually) positive, but the basis is not redundant. A well-constructed blendshape model produces reasonable facial expressions when a few weights (up to five or so) are non-zero, but the models fail when many weights are active (Figure 3.14). Figure 3.15 compares the sparsity of the blendshape encoding to a PCA encoding. The blendshape weights are usually either large or zero, and relatively few weights are active at any point. The PCA representation of the animation has a large number of very small weights. These dense and small weights would be difficult (and laborious) to specify using keyframe animation.

\subsubsection{PCA is not interpretable}

While the first few basis vectors discovered by PCA are often interpretable (for example, the first eigenvector typically reflects the jaw-opening motion), the remaining basis vectors are notoriously difficult to interpret. In this section we explain this lack of interpretability in three ways:

- by intuitive argument: a target such as raise-right-mouth-corner is obviously not orthogonal to jaw-open (the jaw-open motion pulls the mouth corner down slightly).

- by demonstration: Figure 3.16 shows several eigenvectors from a professionally created facial animation, (visualized with the mean added as face meshes). The deformations are global and hard to understand and use.

- By mathematical arguments: 

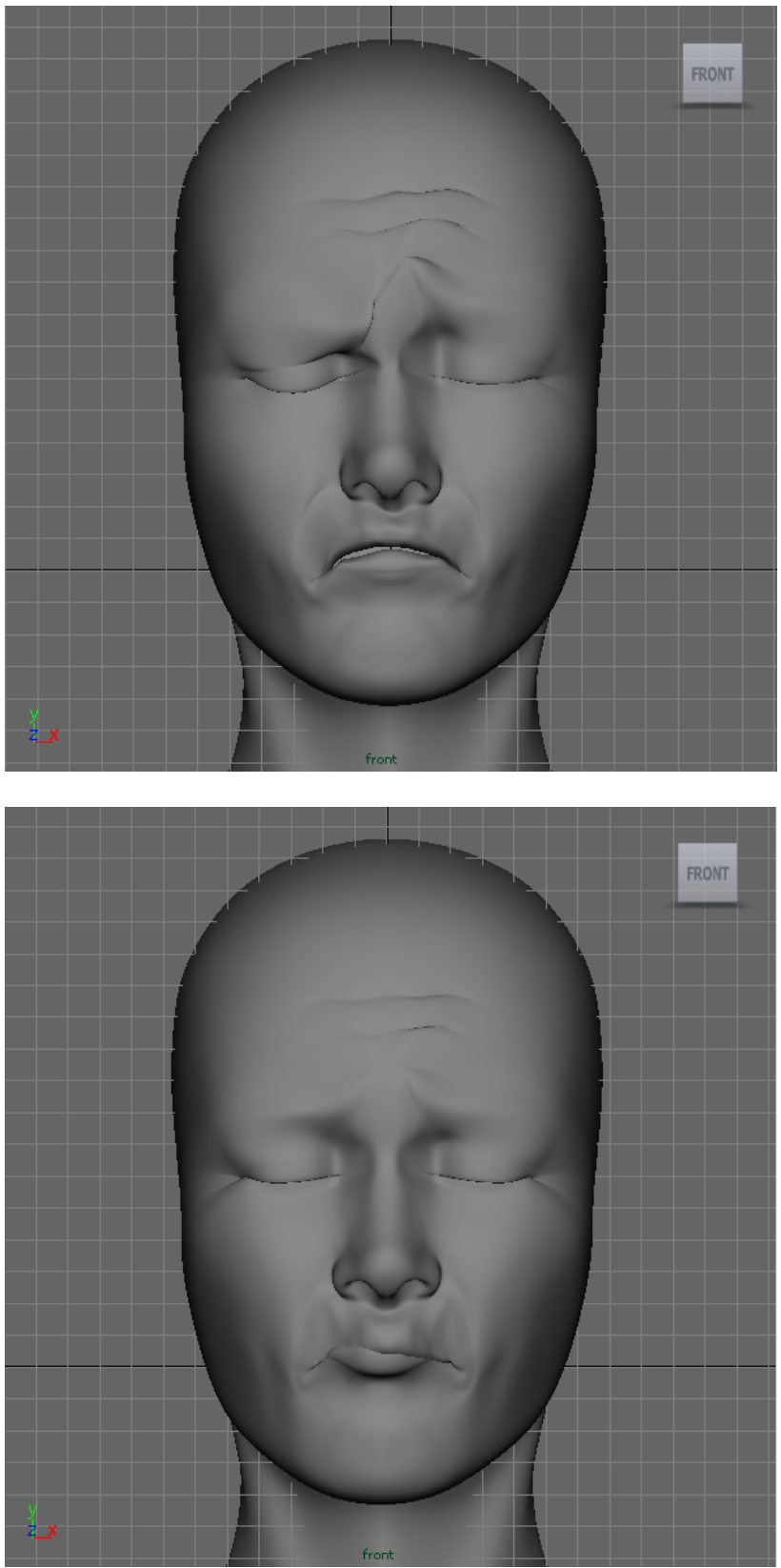

Figure 3.16: PCA basis vectors are difficult to interpret and remember. These are the 9 th and 10th eigenvectors from a professionally produced facial animation. 


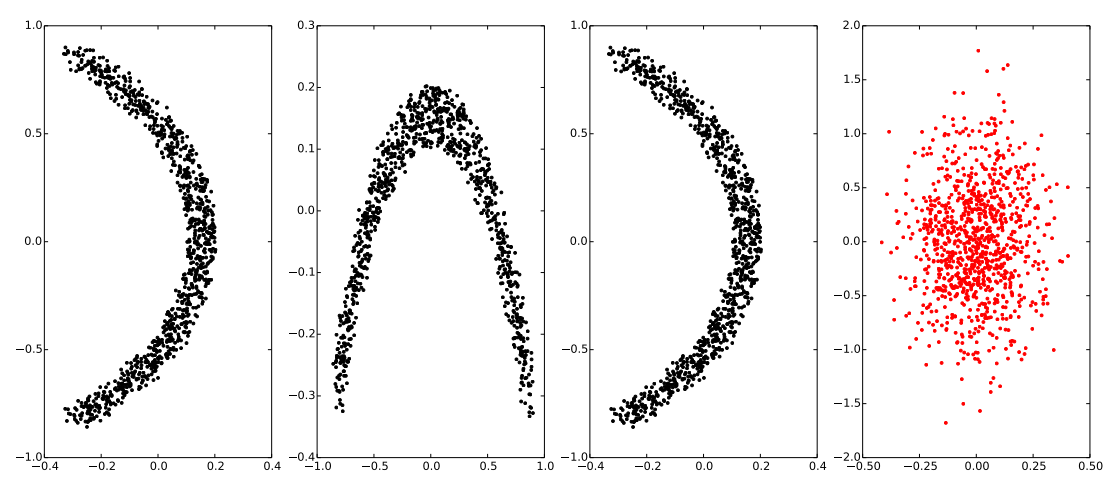

Figure 3.17: PCA is a weak "model" of data. From left to right: a synthetic data set, the PCA coefficients of this data, the rotated PCA coefficients, and random points having the same covariance as the data. While the two eigenvectors and corresponding eigenvalues capture the spread of the data, all the structure ends up in the coefficients. In this two dimensional example the coefficients $\mathbf{c}=\mathbf{U}^{T} \mathbf{f}$ are simply a rotation of the original data points $\mathbf{f}$, since $\mathbf{U}$ is orthogonal. 
1. (An informal variant of the Courant nodal theorem for eigenfunctions of the Laplacian): The second constructed eigenvector is orthogonal to first eigenvector. Consider a hypothetical case where the first eigenvector is everywhere non-negative. In order to be orthogonal, the second eigenvector must have both positive and negative regions over the support of the positive part of the first eigenvector. Thus we see that each eigenvector will tend to have more oscillations than the previous. Note that this argument follows from the orthogonality of the basis, and thus applies equally to PCA variants such as weighted PCA.

2. The eigenvectors are linear combinations of all the variables (this is a motivation for sparse PCA schemes). PCA is the orthogonal basis that minimizes the squared reconstruction error. By the "grouping effect" of least squares [264], if a group of correlated variables contributes to an eigenvector, their contribution tends to be distributed evenly across all variables.

PCA is also quite weak as a means of characterizing or modeling data (Figure 3.17). The data covariance used in PCA uniquely specifies a Gaussian distribution, but non-Gaussian data may also have the same covariance. PCA is thus a viable "model" only if the data is jointly Gaussian, which is not true for either facial proportions ([135]) or facial movement. Figure 3.18 shows scatterplots of several coefficients of the PCA representation of a professionally created animation. The clearly visible structures in this figure illustrate that facial movement is highly non-Gaussian: since the PCA coefficients are a linear function of the data (after removing the mean), and linear transforms preserve Gaussian distribution (indeed transformed non-Gaussian data tends to be more Gaussian than the original), these scatterplots would be Gaussian if the data were Gaussian.

PCA is a particular example of unsupervised learning. Other unsupervised learning approaches have also been applied to facial animation. [50] use Independent Component Analysis (ICA), which tries to extract linear 
components that are statistically independent, a stronger property than the uncorrelated components used by PCA. They show that the extracted components can be categorized in broad motion groups such as speech, emotions, and eyelids. The components can then be used for coarse motion editing such as exaggeration.

\subsubsection{Conversion between blendshape and PCA represen- tations}

A blendshape representation can be equated to a PCA model that spans the same space:

$$
\mathrm{Bw}+\mathbf{f}_{0}=\mathbf{U c}+\mathbf{e}_{0}
$$

where $\mathbf{U}$ and $\mathbf{c}$ are the PCA eigenvectors and coefficients, and $\mathbf{f}_{0}$ and $\mathbf{e}_{0}$ are the neutral face and mean face respectively. The weights can be interconverted as

$$
\begin{aligned}
& \mathbf{w}=\left(\mathbf{B}^{T} \mathbf{B}\right)^{-1} \mathbf{B}^{T}\left(\mathbf{U} \mathbf{c}+\mathbf{e}_{0}-\mathbf{f}_{0}\right) \\
& \mathbf{c}=\mathbf{U}^{T}\left(\mathbf{B w}+\mathbf{f}_{0}-\mathbf{e}_{0}\right)
\end{aligned}
$$

Note that the matrices here (e.g. $\left.\left(\mathbf{B}^{T} \mathbf{B}\right)^{-1} \mathbf{B}^{T} \mathbf{U}\right)$ can be precomputed and are of size $n \times n$. The vectors $\left(\mathbf{B}^{T} \mathbf{B}\right)^{-1} \mathbf{B}^{T}\left(\mathbf{e}_{0}-\mathbf{f}_{0}\right)$ can also be precomputed. Thus converting from weights to coefficients or vice versa is a simple affine transform that can easily be performed at interactive rates on current machines. A blendshape software system can thus internally convert operations into a PCA representation if this is advantageous.

\subsubsection{Probability of a blendshape expression}

Various applications require or can benefit from knowing the "probability" of a facial expression. The Gaussian density leads to simple MAP (maximum a posteriori) computation, so this approach is widely used in 

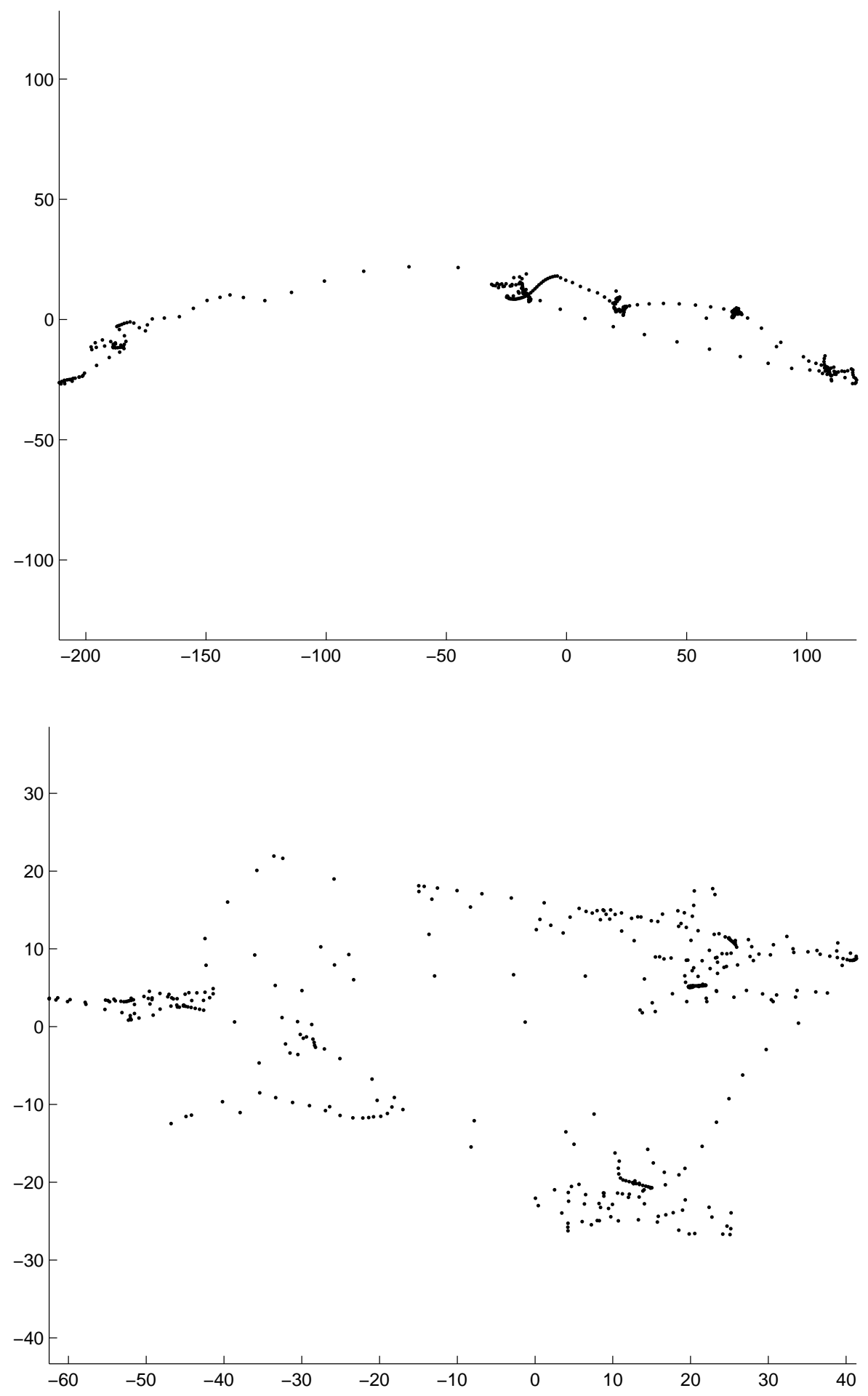

Figure 3.18: Scatterplot of the 1st vs. 3rd PCA coefficients (top) and 2nd vs. 3rd PCA coefficients (bottom) of the professionally-created 405-frame facial animation used in Figure 3.15. The plots show clear non-Gaussian structure. Note that many points are coincident and overlaid in the upper figure. 
many applications. The probability and norm can be used to identify outliers in tracking, and particularly to regularize the inverse problem in direct manipulation facial editing [11].

The correspondence of blendshapes and PCA representations (equation 3.8) gives a simple means to assign a probability to a blendshape expression. The expectation of the square of an individual PCA coefficient is the corresponding eigenvalue:

$$
\begin{aligned}
\mathbb{E}\left[c_{i}^{2}\right] & =\mathbb{E}\left[\mathbf{u}_{i}^{T} \mathrm{ff}^{T} \mathbf{u}_{i}\right] \\
& =\mathbf{u}_{i}^{T} \mathbb{E}\left[\mathrm{ff}^{T}\right] \mathbf{u}_{i}=\mathbf{u}_{i}^{T} \mathbf{C} \mathbf{u}_{i} \\
& =\mathbf{u}_{i}^{T} \lambda_{i} \mathbf{u}_{i} \\
& =\lambda_{i} \quad \text { because }\left\|\mathbf{u}_{i}\right\|=1
\end{aligned}
$$

where $\mathbf{f}$ is a vector representing the face (or other data) with the data mean removed, $\mathbf{u}_{i}$ is a particular eigenvector and $\lambda_{i}$ is the corresponding eigenvalue.

Since the eigenvalues are variances, the multivariate normal density with these variances can be used to assign a probability to a facial expression:

$$
P(\mathbf{c})=\exp \left(-\frac{1}{2} \sum_{i} \frac{c_{i}^{2}}{\lambda_{i}}\right)=\exp \left(-\frac{1}{2} \mathbf{c}^{T} \boldsymbol{\Lambda}^{-1} \mathbf{c}\right)
$$

This also generates a "face norm" $\|\mathbf{f}\|_{\mathcal{K}}$ :

$$
\mathbf{c}^{T} \boldsymbol{\Lambda}^{-1} \mathbf{c}=\left(\mathbf{f}^{T} \mathbf{U}\right)\left(\mathbf{U}^{T} \mathbf{C}^{-1} \mathbf{U}\right)\left(\mathbf{U}^{T} \mathbf{f}\right)=\mathbf{f}^{T} \mathbf{C}^{-1} \mathbf{f}=\|\mathbf{f}\|_{\mathcal{K}}^{2}
$$

The form $\mathbf{f}^{T} \mathbf{C}^{-1} \mathbf{f}$ is the multidimensional counterpart of the argument $f^{2} / 2 \sigma^{2}$ that appears in the one-dimensional Gaussian $\exp \left(-f^{2} / 2 \sigma^{2}\right)$.

There is an important but rarely acknowledged issue with assigning a Gaussian probability to face models however [135]: MAP seeks the mode of the posterior Gaussian. In high dimensions the Gaussian is a heavy tailed distribution, and the mode is a highly atypical point - the interior of the density has almost no volume, and (contrary to some published statements) typical faces drawn from this density will not lie near the mean (Figure 6.4). 


\subsubsection{Outlook}

Although the blendshape idea is extremely simple, careful consideration reveals fundamental issues including high dimensional interpolation (section 3.6, semantic parameterization, and sparse coding. In fact blendshapes provide an interesting "workshop" for discussing general issues of representation and parameterization. The contrast between blendshape representations and principal component analysis is particularly interesting.

\subsection{Generalizations and Future Directions}

We conclude by briefly mentioning two techniques that accomplish nonlinear blending of target shapes. While these are outside of the industry definition of blendshapes, they point the way toward more powerful techniques.

Rather than forming a linear combination of the positions of various target shapes, [227] blend deformation gradients. Specifically, they split the Jacobian into rotation and symmetric factors using polar decomposition and then do linear interpolation in the rotation Lie algebra using the exponential map. The symmetric factor is directly linearly interpolated (symmetric matrices are not a group, but linear interpolation of symmetric matrices preserves the property). This approach might be considered as a nonlinear generalization of the blendshapes.

[150] interprets the original target meshes as a mass spring model and linearly blends edge lengths rather than geometry. This simple approach is able to produce reasonable rotational motion (Figure 3.19) as well as contact and collision effects. 

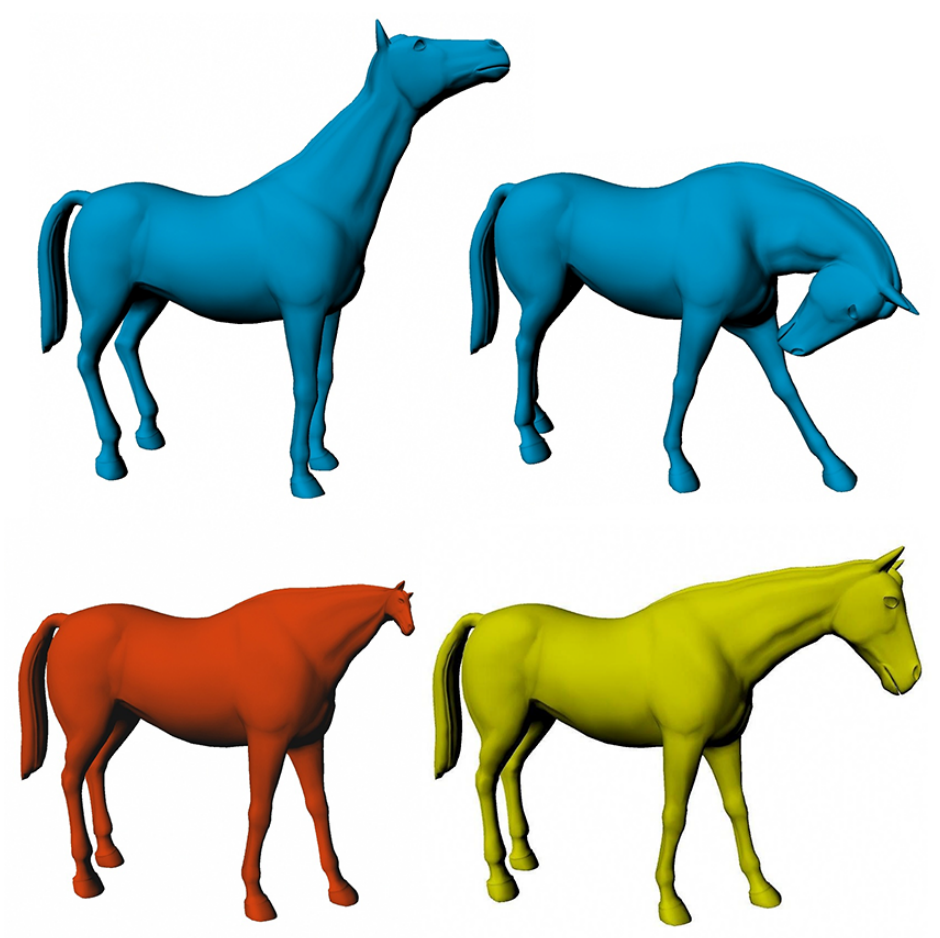

Figure 3.19: From A Blendshape Model that Incorporates Physical Interaction [150]. Top row, two meshes to interpolate. Bottom left, linear interpolation. Bottom right, interpolation of edge lengths followed by a massspring solve. 


\subsubsection{Outlook}

From the viewpoint of current graphics research, the blendshape approach is primitive, and improvements or a successor would be welcome. A direct successor to this approach would need to have several characteristics:

1. The ability to construct the model by directly sculpting or scanning facial expressions,

2. Artists should be able to understand and edit the model's underlying parameters

3. The computation should be relatively lightweight, allowing real-time playback of interactive edits

The algorithms [227, 150] satisfy at least the first two requirements and suggest the way forward.

\subsection{Chapter Summary}

"Blendshapes" are at present the leading approach to realistic facial animation. While most algorithms in graphics industry software and practice can be traced back to original research publications, blendshapes are unusual in that both the original idea and some recent developments such as combination shapes [174] originated outside of academic forums. Despite its simplicity and popularity, the technique has both unresolved limitations and associated open problems. Facial blendshapes are also an attractive "workshop" for exploring issues of representation and parameterization.

This chapter serves as a comprehensive literature survey for Part I of the thesis as well as providing a mathematical analysis of this fundamental technique. It introduces the blendshape approach that is used in chapters 4 and 5. It carefully explores the blendshape representation, including a comparison with PCA, a view in terms of differential geometry concepts 
(manifold and tangent space), and other considerations. The difficulty of high-dimensional smooth interpolation is also considered. As an examplebased method, the blendshape "basis" is interpretable by definition. While this observation is obvious, we feel it deserves to be highlighted. 


\section{Chapter 4}

\section{Accelerating Facial Editing with Statistical Priors}

Although direct manipulation is commonly employed in animating figures through the use of inverse kinematics (IK) (as well as in two-dimensional animation), there has been no analogous "inverse kinematics for blendshape faces". As is the case for body animation, direct manipulation is an inverse problem - when a single facial point is moved, the system must infer the movement of other points. This problem has been approached with several schemes based on custom models using orthogonal (principal component) bases, however a solution that works with the widely-used blendshape representation is desirable.

One challenge in introducing an "inverse kinematics for blendshapes" is the very high dimensionality of the inverse problem. While the facial and body models may both have on the order of 100 degrees of freedom, in the body case various degrees of freedom are isolated by skeleton. For example, moving an arm with IK involves three known degrees of freedom and perhaps five unknown rotations (depending on the particular model); rotations on the other arm, the spine, legs, etc. are not involved. In a blendshape facial model there is no obvious segregation of degrees of freedom, so moving the corner of a mouth may leave on the order of 100 


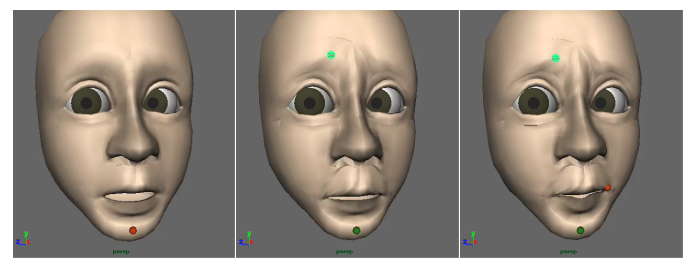

(a)

(b)

$(c)$

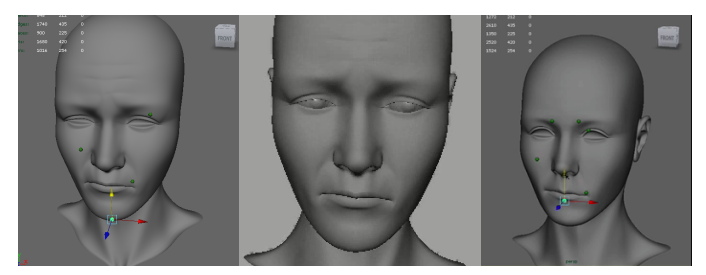

$(d)$ $(e)$

Figure 4.1: (a-c): a blendshape model posed by direct manipulation rather than traditional slider weight editing; (d) a blendshape model posed with a statistical prior, (e) a similar pose from the training animation, (f) an attempt to reproduce this expression without the prior requires more edits and is less successful.

weights to be determined. However, the blendshape model construction reflects the desired semantics of movement, in that a difference in expression roughly corresponds to the distance of slider movement (Figure 3.1). This observation forms the basis for our approach to solving the inverse problem.

In this chapter we first demonstrate that a classic differential manipulation technique can be applied to facial blendshapes. In this approach the inverse problem is implicitly regularized by the nature of the model. We then show that a simple reinterpretation allows the inverse problem to be guided by statistics of prior motion, obtained either from previous animations of the character or from motion capture. The model is internally reparameterized in terms of a principal component basis, and a mode finding step is added to increase the modeling power when sufficient data is available. The result is a sophisticated approach to direct manipulation of blendshape face models that can learn from the statistics of previous motion examples while interoperating with existing animation techniques.

This chapter presents new, unpublished research that builds on the di- 
rect manipulation framework introduced in the author's publication [131].

\section{Chapter Notation}

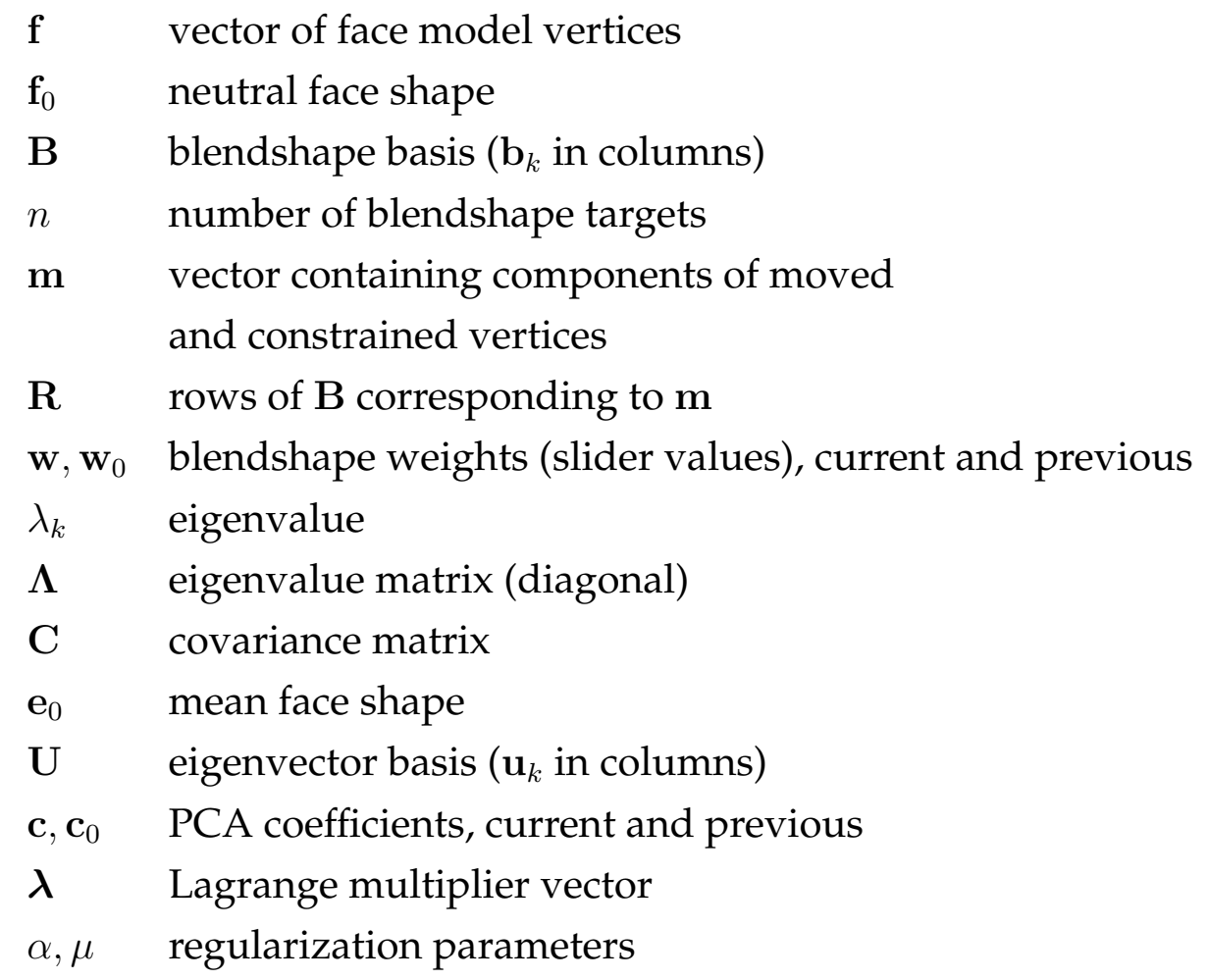

\subsection{Introduction}

As a general principle, it desirable to provide interoperable editing using both 1) direct manipulation, and 2) indirect parameter-level editing of models. Most animation interfaces permit both of these approaches. For example, 2D compositing packages such as Shake allow images to be positioned either by dragging or by adjusting translation values or animation curves, and 3D packages such as Maya and Studio Max allow animated figures to be controlled through either direct manipulation via inverse kinematics or by specification of joint rotations. Yet although blendshapes 
have been common practice in computer facial animation for more than two decades and have been used for lead characters in movies such as Stuart Little, Lord of Rings, and King Kong, there is no existing approach for direct manipulation of blendshapes.

Direct manipulation of a face model is an underconstrained or inverse problem, as is the case with inverse kinematics for the articulated figures, however the number of degrees of freedom to be inferred is usually larger. A realistic blendshape parameterization has up to 100 degrees of freedom or more, while a face model may have 10,000 vertices or more. When a point on the model is moved, tens of parameters need to be inferred for each known degree of freedom.

Several approaches to direct manipulation editing of faces have been formulated for the case of principal component (PCA) models generated from capture data. Unfortunately, it is well known that while the first couple principal components can sometimes be interpreted, the remaining components generally lack semantics [125]. As a result PCA models can be edited by a human artist only through a direct manipulation approach. In current animation practice, however, facial models are manually constructed with semantically defined parameters often inspired by muscles [101, 204]. Further, it is desirable that a facial model be controllable through both direct manipulation and traditional parameter control, as is the case in figure animation where both inverse kinematics and animation curve controls are interchangeably used as needed.

We show that the effect of a single direct manipulation edit is equivalent to a large number of parameter edits in some cases, but the converse is simultaneously true - in some cases a parameter slider does exactly the right thing and reproducing its effect would require multiple direct manipulations.

We approach our problem by first considering basic direct manipulation, and show that a classic differential manipulation technique can be successfully applied to blendshape models. While this simple direct edit- 

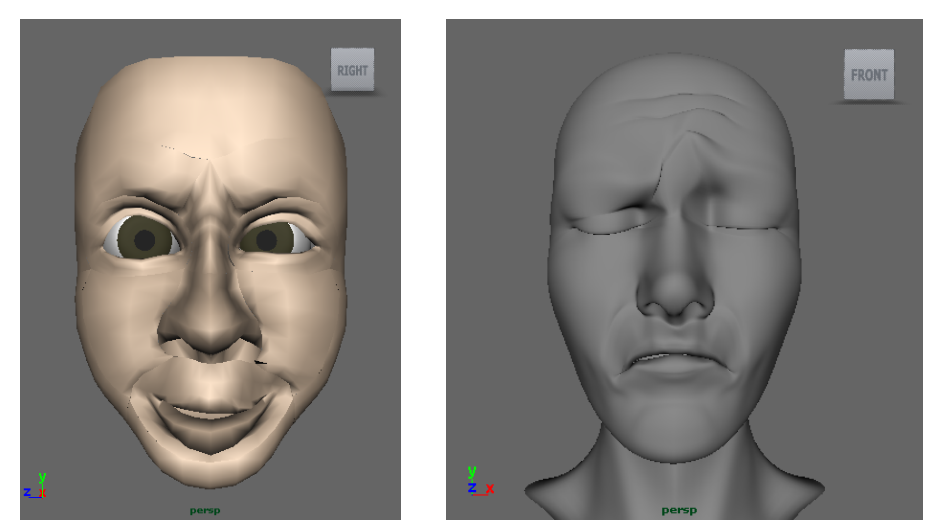

Figure 4.2: The 9th eigenvector from each of two animated models.

ing is well behaved, the underconstrained nature of facial editing leaves room for more refined approaches to inferring the unknown degrees of freedom. A probabilistic reinterpretation of the direct manipulation objective suggests an easy means of incorporating statistical prior information, either from previous character animations or suitable motion capture. To accomplish this, the facial parameters are internally translated to a statistical basis, while preserving the original semantic parameters at the user interface. The resulting approach is simple to implement, efficient (involving only linear system solves), provides direct manipulation editing of standard existing blendshape models, and interoperates with traditional parameter-based keyframe editing.

Figure 4.1 shows the system in operation. The artist simply selects control points or vertices and drags them to desired locations (Figure 4.1 (a-c)). Point configurations that cannot be reached from the blendshape subspace are approximated. Figure 4.1 (d-f) are from the video, illustrating direct manipulation with added prior information from an example animation. With this prior the manipulated model quickly takes on the characteristic "plaintive" expression found in the particular training animation.

Unlike previous work, our approach targets manually sculpted and semantically parameterized blendshape models as used in current anima- 


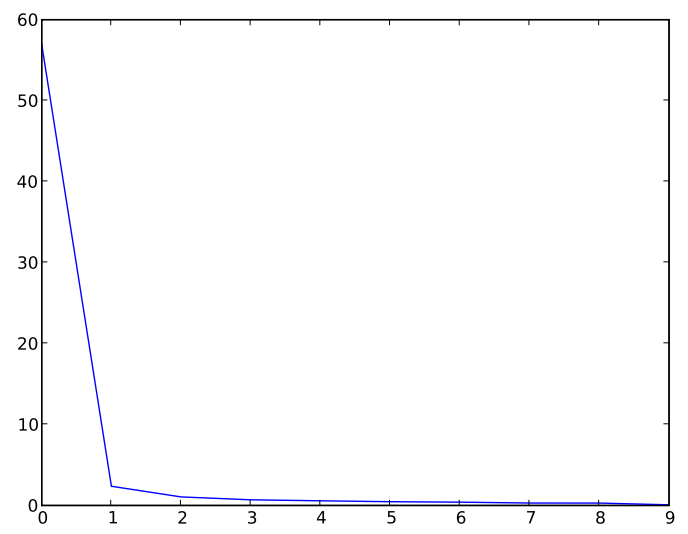

Figure 4.3: First 10 singular values of the blendshape basis of the model in Figure 4.4. Although the basis has rank $n$, there are many directions that "almost" define a null space.

tion practice. We do not assume a PCA basis, custom model creation, or local segmentation, but rather work with any pre-existing blendshape model. Our direct manipulation approach is in the domain of differential manipulation [95] and starts from a similar mathematical formulation. We adapt this to allow suitable constraints and incorporate statistical prior information. We adopt the pin \& drag user interface approach [259], though in the context of facial rather than body animation.

\subsection{Method}

\subsubsection{Direct Manipulation of a Given Model}

In a direct manipulation interface, the animator selects and drags a "target" point on the face model. The system needs to determine the positions of other vertices of the model.

Our solution to this problem is guided by the belief that the blendshape model is created by a skilled artist and thus inherently defines the desired 
range of movement of the character. This leads to two observations:

1. For a face model with $m$ vertices, there are potentially $3 m$ degrees of freedom. However, if the face is a blendshape model with $n$ targets, the face movement is restricted to a subspace of dimension $n$. This is the space of valid shapes as defined by the artist who created the model, and the direct manipulation solution should remain in this space.

2. The space of valid shapes is adequately parameterized by the blendshape weights (sliders). In turn, this implies that Euclidean distance in slider space is at least a first approximation of the semantic distance between face poses.

With these considerations in mind, possible solutions to the direct manipulation problem can be evaluated. One possible solution would be to require all other points move as little as possible. While this has some appeal, it is actually not the animators goal to manipulate the face as little as possible - the animator would rather take large steps, provided they are predictable and controllable.

Instead, we find the movement that causes the smallest change in sliders, subject to interpolating the target point. This solution embodies consideration (2) above, i.e., that the sliders are an appropriate approximate parameterization of the model. This approach also leads directly to the data-driven extension described in section 4.3 .

A direct implementation of this approach is

$$
\frac{1}{2} \min _{\mathbf{w}}\left\|\mathbf{w}-\mathbf{w}_{0}\right\|^{2}+\boldsymbol{\lambda}^{T}(\mathbf{m}-\mathbf{R w})
$$

where $\mathbf{w}_{0}$ are the previous weight values, $\mathbf{m}$ is the vector containing the moved point(s), and $\mathbf{R}$ are the corresponding rows of the $\mathbf{B}$ matrix. Taking the derivative with respect to $\mathrm{w}$,

$$
\mathbf{w}-\mathbf{w}_{0}=\mathbf{R}^{T} \boldsymbol{\lambda}
$$


By pre-multiplying by $\mathbf{R}$ and substituting $\mathbf{m}=\mathbf{R} \mathbf{w}, \boldsymbol{\lambda}$ is obtained as

$$
\boldsymbol{\lambda}=\left(\mathbf{R} \mathbf{R}^{T}\right)^{-1}\left(\mathbf{m}-\mathbf{R w}_{0}\right)
$$

Substituting this into $\mathrm{Eq}$ (4.2) gives the solution for $\mathrm{w}$,

$$
\mathbf{w}=\mathbf{R}^{T}\left(\mathbf{R R}^{T}\right)^{-1}\left(\mathbf{m}-\mathbf{R} \mathbf{w}_{0}\right)+\mathbf{w}_{0}
$$

Mathematically, this technique is the same as the differential manipulation pioneered by [95], although its expression is slightly more complication here because we will need to express both moving and stationary constraints.

Although the approach in Equation (4.1) works well, we choose instead to minimize the distance between the model and the moved point in a least squares sense,

$$
\min _{\mathbf{w}}\|\mathbf{R} \mathbf{w}-\mathbf{m}\|^{2}+\alpha\left\|\mathbf{w}-\mathbf{w}_{0}\right\|^{2}
$$

(with $\alpha=0.1$ in our experiments). The motivations for this will become more apparent in sections $4.2 .2,4.2 .3$ and 4.3 .

\subsubsection{Pinning}

After the animator drags a point, it is desirable to "pin" that point, forcing it to remain stationary as other points are dragged and subsequently pinned [259]. This is easily accomplished in Equation (4.4) simply by appending the pinned points to the vector $\mathbf{m}$, and corresponding rows to the matrix $\mathbf{R}$.

In a particularly simple model, it is possible (though unlikely) that when a sufficient number of points are constrained the $\mathbf{m}-\mathbf{R w}$ constraint will become full rank. In this case the right-hand term in Equation (4.1) would fully specify the facial movement. More generally, (4.1) permits movement only in the null space of the constrained points. However, in practice there are additional directions that are "almost" in the null space, 
corresponding to singular values that are almost but not quite zero (Figure 4.3). Equation (4.4) allows movement these directions, providing additional freedom with only a slight violation of the constraints.

\subsubsection{Haphazard movements}

The artist may drag points in directions and to extents that cannot be reasonably accommodated by the model (Figure 4.4). Note that blendshapes controlled by a standard slider interface have a similar problem, that the sliders can be dragged too far. This problem is presumably not one that plagues experienced artists, and it can be dealt with using an 'undo' mechanism. However, we also cope with the problem by constraining the sliders to a range $[0, t]$ with $t=1$ typically. In addition we incorporate a very mild weight decay regularization term $\mu\|\mathbf{w}\|^{2}$ with $\mu=0.001$ to bias the results away from extreme poses.

Putting these together, our objective is

$$
\min _{\mathbf{w}}\|\mathbf{R} \mathbf{w}-\mathbf{m}\|^{2}+\alpha\left\|\mathbf{w}-\mathbf{w}_{0}\right\|^{2}+\mu\|\mathbf{w}\|^{2}
$$

subject to $\mathbf{w} \in[0, t]$, giving the corresponding quadratic program:

$$
\begin{aligned}
& \qquad \min _{\mathbf{w}} \frac{1}{2} \mathbf{w}^{T} \mathbf{S w}+\mathbf{q}^{T} \mathbf{w} \\
& \text { subject to } \quad \mathbf{G} \mathbf{w} \preceq \mathbf{h} \\
& \text { with } \quad \mathbf{S}=2\left(\mathbf{R}^{T} \mathbf{R}+(\alpha+\mu) \mathbf{I}\right) \\
& \mathbf{q}=-2 \mathbf{R}^{T} \mathbf{m}+\alpha \mathbf{w}_{0} \\
& \mathbf{G}=[\mathbf{I} ;-\mathbf{I}]^{T} \\
& \mathbf{h}=\left[t \mathbf{1}_{n} ; \mathbf{0}_{n}\right]^{T}
\end{aligned}
$$

where $\mathbf{0}_{n}, \mathbf{1}_{n}$ denote zero- and one-vectors of length $n$. This is easily solved at interactive frame rates on current machines. Figure 4.4 shows the results of Equation (4.5) versus Equation (4.4) on an unreasonable movement. 

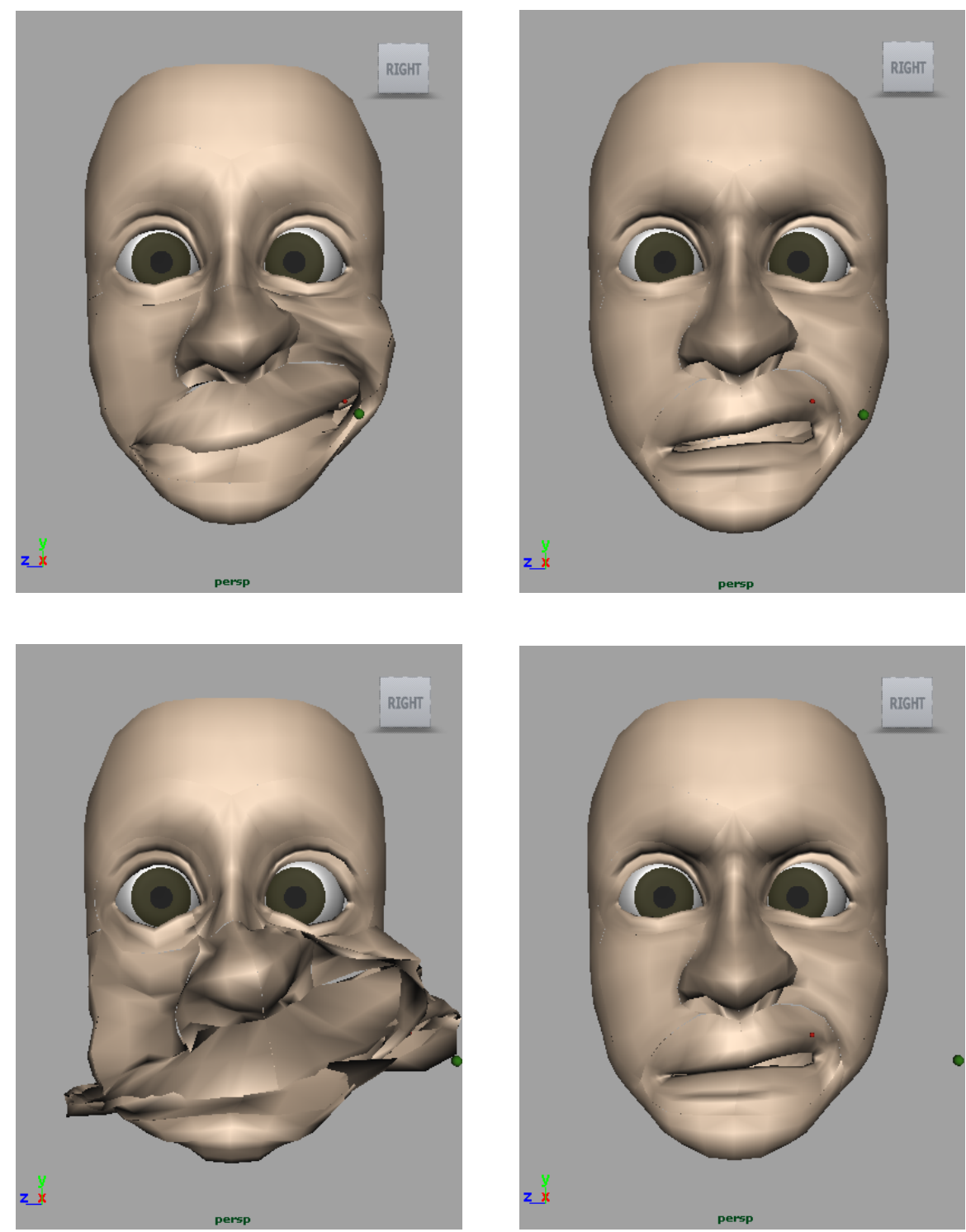

Figure 4.4: Left column: model behavior with unconstrained weights as the target point is dragged to an unreasonable location. Right column: corresponding constrained solutions to this haphazard drag. The green point is the target point location desired by the user. 


\subsubsection{Implementation}

Our work is targeted to professional animators rather than novice users. Current animation practice employs large software packages such as Maya that have reusable components implementing most of the functionality we need, including basic blendshapes, spline surfaces, constraining of points to surfaces, and interaction in a perspective window. We further assume that the animator is skilled in the use of this software. In particular, in the case of spline models (such as those shown here) we currently provide direct manipulation of the spline control vertices. This is standard practice in manipulating spline surfaces and does not present any challenge for skilled artists.

\subsubsection{Relative power of direct manipulation versus para- metric control}

A single direct manipulation vertex move is generally equivalent to a large number of slider movements, but the converse is also true. This can be seen from Equation (4.3), which is of the form

$$
\mathbf{w}=\mathbf{X m}+\mathbf{k}
$$

with $\mathbf{X}=\mathbf{R}^{T}\left(\mathbf{R R}^{T}\right)^{-1}$. Consider the effect of $\mathbf{m}$ containing a single pinned vertex. The $\mathbf{X}$ matrix is more dense than $\mathbf{R}$ due to the convolution effect of the matrix multiply and the bandwidth increase due to the matrix inverse, so the single mouse move will result in many weight (slider) movements, as illustrated experimentally in Figure 4.5. Conversely, solving Equation (4.6) for $\mathbf{m}$ in terms of $\mathbf{w}$ involves an inverse and thus a single slider movement is the equivalent of multiple mouse movements. As a consequence, both direct manipulation and slider control are advantageous in different circumstances and both should be provided. The sliderbased interface generally assumes a highly skilled artist who is fully familiar with the characteristics of the model. We assume that this person 


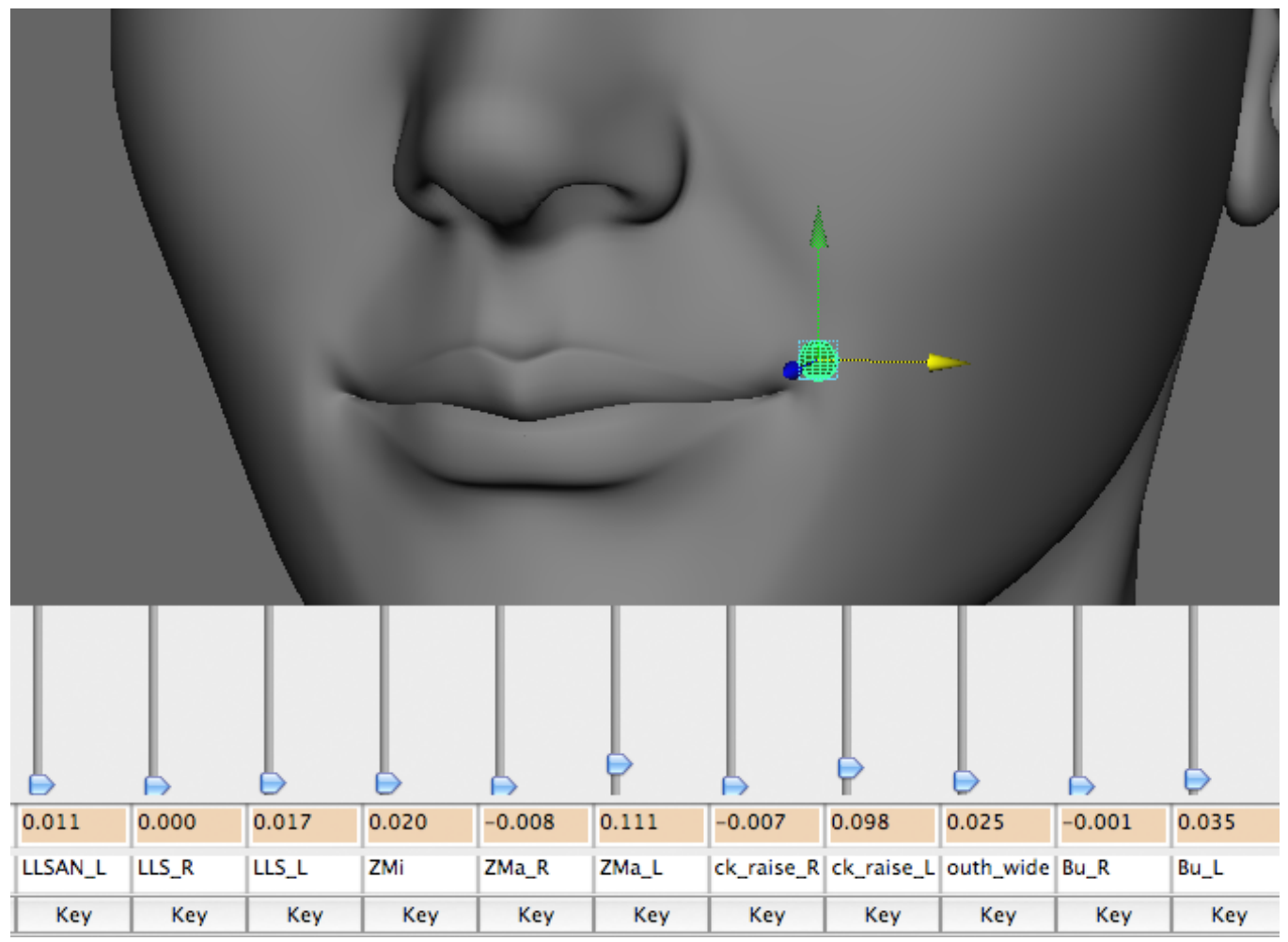

Figure 4.5: With a single vertex pinned (top), direct movement of a second vertex is the equivalent of many small slider movements (bottom). However the converse is equally true: a single slider movement is in some cases the equivalent of multiple direct manipulations. Both approaches are desirable. 
will also learn when sliders are appropriate.

\subsection{Incorporation of Prior Data}

The previous solution assumes that the model's parameterization is appropriate for direct manipulation. Specifically, it assumes that changes in facial expression are proportional to Euclidean distance in "slider space". This is approximately true due to the construction of the model. However, the model has conflicting goals, in that it must reproduce the full range of movement (including unlikely movements) as well as provide a parameterization reflecting typical movements. Also, typically each target is designed so that a slider range of $[0,1]$ is appropriate for most uses. Thus, distance in slider space is only approximate.

If movement data is available, we can do better. This data may come from an existing animated performance of the character, or from suitable motion capture. The former possibility is particularly interesting, in that our animation system then "learns" how to appropriately solve the inverse problem from previous animation.

The formulation in Equation (4.4) can be seen as minimizing a sum of data fidelity $\|\mathbf{R w}-\mathbf{m}\|^{2}$ and prior terms, and thus suggests a loose Maximum A Posteriori (MAP) interpretation. MAP seeks the most likely parameter values given the data (in our case, constraints), $\arg \max _{\mathbf{w}} P(\mathbf{w} \mid \mathbf{m})$, By Bayes theorem (and ignoring the normalizing constant) this is proportional to $P(\mathbf{m} \mid \mathbf{w}) P(\mathbf{w})$. Taking the (negative) log yields two terms corresponding to the data fidelity and prior.

To develop this data+prior approach to the inverse problem, a probability model is needed. We start with a principal component model, for two reasons: 1) it provides a simple and efficient prior, 2) the data compression properties of PCA complement an easy extension to incorporating higher order statistics, as described in section 4.3.1. While at first sight PCA might seem an unusual choice, in fact PCA is commonly and suc- 
cessfully employed as a simple probabilistic model [69, 54, 105]. The PCA model will be denoted

$$
\mathbf{f}=\mathbf{U} \mathbf{c}+\mathbf{e}_{0}
$$

where $\mathbf{U}$ contains eigenvectors of the data covariance matrix

$$
\mathbf{C}=\mathbb{E}\left[\left(\mathbf{x}-\mathbf{e}_{0}\right)\left(\mathbf{x}-\mathbf{e}_{0}\right)^{T}\right]
$$

in its columns, and $\mathbf{e}_{0}$ is the mean face shape (the mean is ignored in the prior computations below since it does not vary with the shape coefficients). With the data thusly considered as being random, the PCA eigenbasis is deterministic, but the model coefficients are again regarded as random. A prior on an individual PCA coefficient can be defined as follows:

$$
\begin{aligned}
E\left[c_{i}^{2}\right] & =E\left[\mathbf{u}_{i}^{T} \mathbf{x x}^{T} \mathbf{u}_{i}\right] \\
& =\mathbf{u}_{i}^{T} E\left[\mathbf{x} \mathbf{x}^{T}\right] \mathbf{u}_{i}=\mathbf{u}_{i}^{T} \mathbf{C} \mathbf{u}_{i} \\
& =\mathbf{u}_{i}^{T} \lambda_{i} \mathbf{u}_{i} \\
& =\lambda_{i} \quad \text { because }\left\|\mathbf{u}_{i}\right\|=1
\end{aligned}
$$

(with $\mathrm{x}$ now denoting the mean-removed data).

With this prior we can formulate the problem in the reproducing kernel Hilbert space (RKHS) $\mathcal{K}$ derived from the data covariance [241], and make use of the RKHS norm to regularize the solution,

$$
\min _{\mathbf{f} \in \mathcal{K}}\|\overline{\mathbf{U}} \mathbf{c}-\mathbf{m}\|^{2}+\alpha\|\mathbf{f}\|_{\mathcal{K}}^{2}
$$

where \|\|$^{2}$ is the usual squared norm, the \|\|$_{\mathcal{K}}$ norm is

$$
\|\mathbf{f}\|_{\mathcal{K}}^{2}=\mathbf{f}^{T} \mathbf{C}^{-1} \mathbf{f}=\left(\mathbf{f}^{T} \mathbf{U}\right)\left(\mathbf{U}^{T} \mathbf{C}^{-1} \mathbf{U}\right)\left(\mathbf{U}^{T} \mathbf{f}\right)=\mathbf{c}^{T} \boldsymbol{\Lambda}^{-1} \mathbf{c}=\sum_{i} \frac{c_{i}^{2}}{\lambda_{i}}
$$

and $\overline{\mathbf{U}}$ contains the rows of $\mathbf{U}$ corresponding to the constrained points.

As a result, we can replace the $\|\mathrm{w}\|_{2}^{2}$ norm in equations (4.4)-(4.5) with the weighted norm $\|\mathbf{c}\|_{\Lambda}^{2}=\mathbf{c}^{T} \boldsymbol{\Lambda}^{-1} \mathbf{c}$, and our objective is now in the data+prior form

$$
\min _{\mathbf{c}}\|\overline{\mathbf{U}} \mathbf{c}-\mathbf{m}\|^{2}+\alpha\left(\mathbf{c}-\mathbf{c}_{0}\right)^{T} \boldsymbol{\Lambda}^{-1}\left(\mathbf{c}-\mathbf{c}_{0}\right)
$$




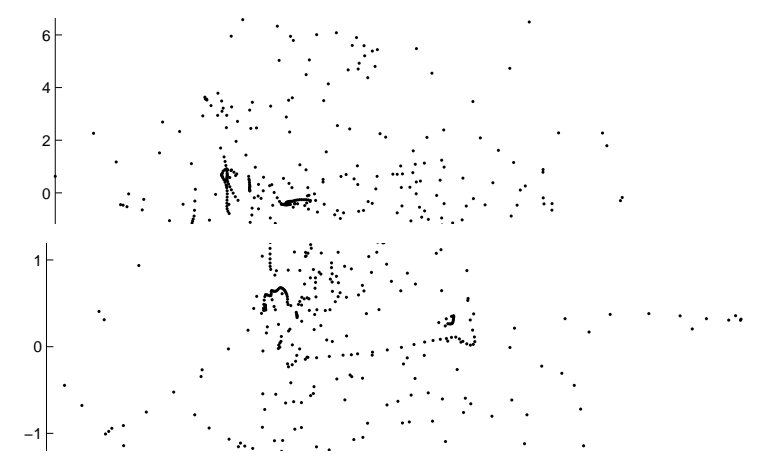

Figure 4.6: Scatterplot of selected PCA coefficients of a professional animation with 405 frames. Top: $c_{5}$ versus $c_{6}$, bottom: $c_{10}$ versus $c_{11}$. (also see Figure 3.18). In several animations such as this we found that the lowernumbered coefficients are significantly non-Gaussian.
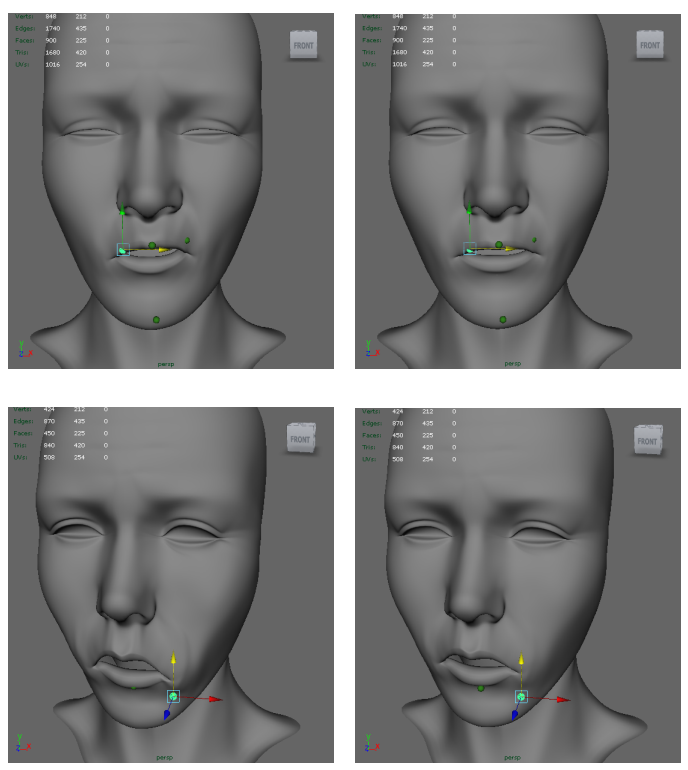

Figure 4.7: Examples of mode snapping. Left column: using Equation (4.8). Right column: applying mode snapping, Equation (4.9). 
This prior has the effect of preferring movements in face pose in directions that had larger variance in the training data. Following standard practice [69, 145] we limit the range of $\mathbf{c}$ to three standard deviations, $\left|\mathbf{c}_{k}\right| \leq \pm 3 \sqrt{\lambda_{k}}$. Note that Equation (4.8) is directly parallel to Equation (4.4) and can be implemented with a small modification of the same program code.

While the objective $(4.8)$ is optimized by the system internally, it is necessary to convert to and from the semantic blendshape slider representation presented to the animator. In general the coefficients for an existing face pose can be obtained as $\mathbf{c}_{0}=\mathbf{U}^{T} \mathbf{f}$ however more efficient solutions are available. The vector $\mathbf{c}_{0}$ is only unknown when a new animation is started, and in this case the default zero initial slider values imply zero coefficients as well. At any other point, the animator has either performed a direct manipulation, with a new $\mathbf{c}$ value is found through optimizing Equation (4.8), or has adjusted the slider weights w directly, requiring the corresponding $\mathrm{c}$ to be found. The vectors $\mathrm{c}$ and $\mathrm{w}$ are related through

$$
\mathbf{U} \mathbf{c}+\mathbf{e}_{0}=\mathbf{B w}+\mathbf{f}_{0}
$$

so they can be interconverted as

$$
\begin{aligned}
& \mathbf{w}=\left(\mathbf{B}^{T} \mathbf{B}\right)^{-1} \mathbf{B}^{T}\left(\mathbf{U} \mathbf{c}+\mathbf{e}_{0}-\mathbf{f}_{0}\right) \\
& \mathbf{c}=\left(\mathbf{U}^{T} \mathbf{U}\right)^{-1} \mathbf{U}^{T}\left(\mathbf{B w}+\mathbf{f}_{0}-\mathbf{e}_{0}\right)
\end{aligned}
$$

Note that the matrices here (e.g. $\left.\left(\mathbf{B}^{T} \mathbf{B}\right)^{-1} \mathbf{B}^{T} \mathbf{U}\right)$ can be precomputed and are of size $n \times n$. The vectors $\left(\mathbf{B}^{T} \mathbf{B}\right)^{-1} \mathbf{B}^{T}\left(\mathbf{e}_{0}-\mathbf{f}_{0}\right)$ can also be precomputed. Thus converting from weights to coefficients or vice versa is a simple affine transform requiring less than 1/1000 second on a current laptop machine.

\subsubsection{An extension to higher order statistics}

When used as a probabilistic model, PCA reproduces the mean and covariance of the data. Data that is jointly Gaussian is fully specified by its mean and covariance. While Gaussian distributions are expected for phenomena that are the sum of many independent causes, many other types 
of data do not have Gaussian statistics. In these cases there is higher order structure in the data that is not captured by the mean and covariance. This structure appears in the distribution of PCA coefficients (Figure 4.6).

Ideally, we would like a probabilistic model that scales to incorporate these higher order statistics, provided sufficient data is available to support the additional parameters. PCA has been extended to capture such higher order statistics by incorporating a nonlinearity either before or after the PCA itself. Kernel PCA [209] places the nonlinearity first, nonlinearly transforming the data into a "feature space" before performing PCA. The choice of an appropriate nonlinearity that exposes relevant features is data dependent. This is one drawback of KPCA: the effect of the nonlinearity is usually not obvious, so the experimenter is faced with the task of blindly exploring a function space, and the chosen function may not reveal all features important to the problem.

We start from the idea of using a kernel density modeling of the PCA coefficient distribution, thus placing the a non-Gaussian modeling step after the PCA. In the case of a highly non-Gaussian distribution (Figure 4.6) we can start from the solution obtained in Equation (4.8) and "crawl uphill" to a local mode, obtaining a more probable solution that reflects the non-Gaussian statistics. The combination of PCA and kernel density would be advantageous in that the kernel density captures statistics that are averaged over by the PCA, while the PCA offsets the curse of dimensionality that prevents the application of kernel density methods in high dimensions.

Unfortunately, the PCA is unlikely to reduce the dimensionality sufficiently to directly allow this approach. For example, if we assume the PCA can produce a 10:1 dimensionality reduction for the purposes of prior density modeling, a blendshape model with 100 targets will still result in a 10dimensional space to be modeled with the kernel density. In fact, on some facial models PCA is only able to produce a 3:1 compression with acceptable quality loss (section 3.4.6), which is not surprising since blendshapes 
are already a somewhat compact representation.

Our goal is not to fully and accurately modeling the coefficient density, however - we want simply to estimate the local mode, remembering that in the interactive application a less-than-ideal estimate will continue to be refined, or can be immediately removed with an undo. After experimenting with several kernel density schemes, we settled on a particularly simple approach: the point $\mathbf{c}$ found in Equation $(4.8)$ is moved toward the mean $\overline{\mathbf{c}}$ of points within the local neighborhood while respecting the imposed constraints,

$$
\min _{\mathbf{c}}\|\overline{\mathbf{U}} \mathbf{c}-\mathbf{m}\|^{2}+\beta(\overline{\mathbf{c}}-\mathbf{c})^{T} \boldsymbol{\Lambda}^{-1}(\overline{\mathbf{c}}-\mathbf{c})
$$

where $\beta$ is a regularization constant similar to $\alpha$ in Equation 4.8. We call this approach "mode snapping", meaning that the coefficients are snapped toward the nearest peak of the training coefficient density function.

Mode snapping resembles one iteration of the mean-shift mode-finding procedure [68] and has the advantage that the code from Equation (4.8) can be directly reused. The intuition for applying Equation (4.8) followed by (4.9) rather than immediately using (4.9) is analogous to removing the mean and linear trend (or other parametric model fit) before performing a non-parametric modeling step. In common with most kernel densityrelated procedures the neighborhood width must be experimentally selected. After looking at coefficient scatterplots we selected $\sigma_{i} / 3$ as a width in each dimension, and perform the nearest neighbor search in the threedimensional space $i \leq 3$. In the case where no points are within the neighborhood, the original estimate $\mathrm{c}$ is not changed.

\subsection{Results}

Figure 4.1 (a)-(c) show a blendshape model posed by our direct manipulation at interactive rates, using three constraints ("moves"). Producing an expression such as this using traditional slider (blendshape weight) 
editing would typically require several iterations of weight adjustment, as well as some trial-and-error if one is not intimately familiar with the model. Figure 4.1(d) shows a model posed by our method with four moves and making use of a prior from a training animation. Figure 4.1 (e) is an expression resembling Figure 4.1(d) that appears in the training animation. Note that Figure4.1(d) is created from scratch; the only information taken from the training animation is the covariance matrix. In Figure 4.1(f) we attempt to mimic this same expression without using a prior; six moves are not sufficient. Comparing (d) with (f) illustrates the capability of our method to make use the statistics of example animation to help the animator stay "on character". Figure 4.7 shows examples where face pose is improved by adding the mode-snapping technique from section 4.3.1 (mode-snapping does not produce a clear difference in every case however).

Finding the right amount of the training data for Equation 4.8 is one unknown factor. Our intuition is that a simple model such as PCA looses its effectiveness when it is required to simultaneously represent too much (non-Gaussian) variety of pose, for the same reason that a line may fit a small portion of a curve but does a poor job in fitting the whole curve. Thus the use of training data may be most effective when it is limited to a small range of poses that also resemble those desired in the animation being created. It is not critical to optimize this choice however: we target professional animators, who have the skill to determine when and if it is appropriate to use training, as well as what data should be used. The introduction of direct manipulation is the major benefit of our system.

\subsection{Chapter Summary}

Blendshape facial models are used in leading animation and film projects, involving thousands of hours of animator labor on each project. Skilled and efficient use of a blendshape model requires the animator to effec- 
tively memorize the function of each of the 50-100 sliders typical of current models. Furthermore, even locating the correct slider to move requires visual search (Figure 3.3. As a rough estimate, professional 3D animation requires on the order of an hour of animator time to produce each second of animation. As is the case with inverse kinematics for figure animation, a direct manipulation interface can significantly accelerate this work in some or perhaps most cases. Yet although direct manipulation for figure animation has been available for many years, a direct manipulation solution for facial blendshape models has not been previously proposed.

Our solution to this problem is easy to implement, efficient (requiring only linear system solves), and has several clear benefits:

- it uses standard blendshape models,

- it allows for the incorporation of "prior" data if desired, with very little extra programming.

- it interoperates with standard slider-based blendshape animation.

In general the inverse solution provided by a direct manipulation drag will be closer to the desired expression, but it will not be exactly as desired. Our approach benefits from a "no loss" principle: if the result of dragging a point does not give the desired results, the animator can position additional points, or simply undo the movement. Each moved point serves as a "pin" to constrain subsequent drags [259], allowing rapid successive refinement of the facial expression.

While the direct manipulation interface itself is clearly efficient in many cases, the incorporation of prior data directs the free parameters toward poses that were more common in the training data, thus "in expectation" saving additional work. Note that some of these effects are subtle (sometimes extremely so), but this is the nature of facial animation - the difference in facial expressions between two expressions such as "afraid" and "alarmed" can be quite small, yet it is the animator's task to recreate these fine differences. 
A further contribution of this chapter is the general idea of using kernel density modeling of the PCA coefficient distribution. Kernel density estimation can represent arbitrary data, but works poorly in high dimensions [210]. On the other hand PCA is a useful dimensionality reduction tool, but has very limited modeling power. The technique proposed in section 4.3.1 combines these techniques in a way that each compensates for the other's weakness. 
102 CHAPTER 4. FACIAL EDITING WITH STATISTICAL PRIORS 


\section{Chapter 5}

\section{A Space-Time Approach to Expression Cloning}

Previous chapters have investigated the blendshape representation and algorithms to pose the face at individual frames. The ability to pose a facial model at individual frames is sufficient to produce keyframe animation. We now turn to a core problem in producing facial animation using motion capture, expression cloning [171]. This chapter forms the basis for the author's contribution to the publication [214].

\subsection{Introduction}

Motion capture is most often used to drive a character different from the actor producing the motion, for the simple reason that if the motion of the actor was desired, it might be sufficient to use a camera! (There are clear exceptions to this principle however: sometimes it is required to give the actor dramatic movement that would not be achievable with a real camera, as in the virtual actors scenes in The Matrix sequels [37].)

The motion capture actor will be referred to as the source, and the source motion is used to drive a target character. The target might be an older or younger version of the actual actor, as in the movie The Curious 
Case of Benjamin Button, or it may be a fantasy character, as in the movies Polar Express, Avatar, and The Hobbit series [213].

The expression cloning problem was introduced into academic research in [171]. Section 3.5.3 briefly surveys the literature on this topic. A common aspect of all previous approaches to the expression cloning problem is that they consider each frame independently.

This chapter introduces a new approach to expression cloning, in which the problem is solved by considering the entire motion at once. Because it is motion that is being transferred from one character to another, it makes sense in abstract to consider the entire motion rather than isolated frames. However, a specific benefit and algorithm for doing so is needed.

We start from the obvious principle that "the movement should be similar". This is formalized by requiring that the velocity (temporal derivatives) of the motion of each vertex should be similar between the source and target in a least squares sense. If the target is able to exactly reproduce the motion of the source, this would give an identical result to solving each frame independently. However, in general the target is not able to exactly reproduce the motion of the source, due to differing geometry and resulting differences in the realistic range of movement. For example, if a human actor drives the motion of a virtual duck character, it may be that certain mouth shapes cannot be reproduced without violating the duckness of the character.

\subsection{Formulation as a Poisson Equation}

The principle will be formalized as a "gradient domain" or boundary value problem. Restating, the goal is to minimize the differences in the movement of the source and target faces. The movement is $d$ (position) $/ d$ (time), so this requests that the time derivatives of the source and target be matched - a gradient domain problem. The boundary constraint is known sourcetarget correspondence at the beginning and end of the animation - typi- 
cally at the beginning and end both source and target will be in a neutral position. This setup, i.e. saying that the gradients are known, and the value is known only on the boundary, resembles the Poisson equation.

For a single coordinate of a vertex this would be

$$
\min _{T} \int\left(T^{\prime}(t)-S^{\prime}(t)\right)^{2} d t \quad \text { subject to } S, T=\text { neutral at } t=0, t=\text { end }
$$

where $T^{\prime}(t)$ is the time derivative of the target position $T(t)$ and likewise for $S(t)$. Now say that $\mathbf{s}, \mathbf{t}$ are the source and (unknown) target positions, encoded into vectors. $\mathbf{D}$ is the derivative operator encoded into a matrix, i.e.

$$
\mathbf{D}=\left[\begin{array}{cccc}
-1 & 1 & & \\
& -1 & 1 & \\
& & -1 & 1 \\
& & & \ldots
\end{array}\right]
$$

When applied to a vector of values over time, this subtracts the previous value from the current one, i.e. it is a first finite difference.

The "movement should be similar" objective can then be expressed as

$$
\min _{\mathbf{t}}\|\mathbf{D}(\mathbf{t}-\mathbf{s})\|^{2}=(\mathbf{t}-\mathbf{s})^{T} \mathbf{D}^{T} \mathbf{D}(\mathbf{t}-\mathbf{s})
$$

Noting that $\mathbf{D}^{T} \mathbf{D}=\mathbf{L}$ is a second finite difference operate (discrete onedimensional Laplacian), we rewrite as

$$
=\mathbf{t}^{T} \mathbf{L} \mathbf{t}-2 \mathbf{t}^{T} \mathbf{L s}+\mathbf{s}^{T} \mathbf{L} \mathbf{s}
$$

Taking the derivative with respect to $\mathbf{t}$ gives $\mathbf{L t}=\mathbf{L s}$, i.e. When applied to a single vertex, the target movement will just reproduce the source movement exactly. As discussed above, however, when applied to a whole model it will not be possible to exactly transfer the velocities, due to the differing source-target geometries. 


\subsection{Formulation for Blendshapes}

The preceding description is straightforward but abstract. We now reformulate it for the specific case where the target is a blendshape model.

In order to help check our computations we introduce a notation for the dimensions: $\mathbf{M}_{\cdot a, b}$, meaning matrix $\mathbf{M}$ has dimensions $a \times b$. The needed dimensions are: $. v 3=$ number of vertices $* 3, . m=$ number of blendshape modes (targets), . $f=$ number of frames (to be used below).

Notate the blendshape face as

$$
\mathbf{f}+\mathbf{n}=\mathbf{B w}+\mathbf{n}
$$

where $f_{. v 3,1}$ is the resulting face mesh with the vertices "vectorized" into a column vector in some order such as $x x x x x \ldots y y y y y . . . . z z z z z . \mathbf{B}_{. v 3, . m}$ is the blendshape basis matrix; its columns contain the blendshape targets. $\mathbf{w}_{. m, 1}$ is the weight or blendshape slider vector, with values typically between 0,1 . $\mathbf{n}$ is the neutral face shape, so the entries in $\mathbf{B}$ are deltas from this. In the following math we will ignore $\mathbf{n}$ and assume that it will be added back in at the end.

A whole facial performance can then be notated

$$
\mathbf{F}_{. v 3, . f}=\mathbf{B}_{. v 3, . m} \mathbf{W}_{. m, . f}
$$

with $\mathbf{F}$ containing the a mesh for each frame in its columns, and $\mathbf{W}$ containing the weights for each frame in its columns.

Denote the source performance as $\mathbf{S}$ and the target blendshape performance as

$$
\mathbf{T}=\mathbf{B W}
$$

where columns of $\mathbf{S}, \mathbf{T}$ contain the face meshes over time, B is the blendshape models for the target, and $\mathrm{W}$ are the blendshape weights for the target.

The following derivation assumes that the source and target have the same number of vertices, which is unrealistic. However, if an existing expression cloning algorithm can identify a subset of corresponding vertices, 
then this derivation applies, with $\mathbf{S}, \mathbf{T}$ and $\mathrm{B}$ now containing the subset of vertices rather than the whole face.

The overall statement "source and target should move similarly" is now

$$
\mathrm{DS} \approx \mathrm{DT}
$$

or

$$
\mathrm{DS} \approx \mathrm{DBW}
$$

We want to solve for $\mathbf{W}$. This is

$$
\min _{\mathbf{W}} \operatorname{tr}(\mathbf{D T}-\mathbf{D S})^{T}(\mathbf{D T}-\mathbf{D S})
$$

where $\mathbf{D}$ is a derivative operator that works on all vertices at once (to be worked out below). Note that the $\operatorname{tr} \mathbf{M}^{T} \mathbf{M}$ pattern is equivalent to the sum of the squared entries of the matrix $\mathrm{M}$ (i.e. is the Frobenius norm).

Inserting $\mathbf{T}=\mathbf{B W}$ this is

$$
\min _{\mathbf{W}} \operatorname{tr}(\mathbf{D B W}-\mathbf{D S})^{T}(\mathbf{D B W}-\mathbf{D S})
$$

\subsection{Temporal Derivative of all Vertices as a Ma- trix Expression}

We wish to take the difference between the source and target faces and return a matrix with rows containing the temporal derivative of a component $(x, y$, or $z)$ of a vertex at the frame indicated by the particular column. To do this we can use the same $\mathbf{D}$ matrix as before, but it has to operate on the transpose of the $\mathbf{S}$ and $\mathbf{T}$ matrices, so that each row expresses the derivative of the whole face at a particular time, and each column has a temporal history of a component $(\mathrm{x}, \mathrm{y}$, or $\mathrm{z})$ of a vertex.

To simplify notation, we will rename $\mathbf{S} \Leftarrow \mathbf{S}^{T}, \mathbf{B} \Leftarrow \mathbf{B}^{T}, \mathbf{W} \Leftarrow \mathbf{W}^{T}$ and remember the altered meaning. The dimensions of the renamed variables 
are

$$
\mathbf{D}_{. f, f} \mathbf{W}_{. f, . m} \mathbf{B}_{. m, v} \quad-\quad \mathbf{D}_{. f, f} \mathbf{S}_{. f, v}
$$

Expanding the objective,

$\operatorname{tr}(\mathbf{D W B}-\mathbf{D S})^{T}(\mathbf{D W B}-\mathbf{D S})=\operatorname{tr} \mathbf{B}^{T} \mathbf{W}^{T} \mathbf{D}^{T} \mathbf{D W B}-2 \mathbf{S}^{T} \mathbf{D}^{T} \mathbf{D W B}+\mathbf{S}^{T} \mathbf{D}^{T} \mathbf{D S}$

and $\mathbf{D}^{T} \mathbf{D}$ is a discrete second finite difference (one dimensional Laplacian) matrix L, so

$$
\min _{\mathbf{W}} \operatorname{tr} \mathbf{B}^{T} \mathbf{W}^{T} \mathbf{L W B}-2 \mathbf{S}^{T} \mathbf{L W B}+\mathbf{S}^{T} \mathbf{L S}
$$

Taking the derivative with respect to $\mathbf{W}$, this gives

$$
\mathbf{L W B B}^{T}=\mathbf{L S B}^{T}
$$

of dimension

$$
\mathbf{L}_{. f, . f} \quad \mathbf{W}_{. f, . m} \quad \mathbf{B}_{. m, v 3} \quad \mathbf{B}_{. v 3, . m}^{T} \quad=\mathbf{L}_{. f, . f} \quad \mathbf{S}_{. f, v 3} \quad \mathbf{B}_{. v 3, . m}^{T}
$$

The dimension of $\mathbf{B B}^{T}$ is $(. m, . v 3 \times . v 3, . m)$ and so will be invertible if $. m<. v 3$ which will generally be the case (e.g. a typical blendshape model may have $. m=100$ targets but $. v 3=30,000$ vertex components). So

$$
\mathbf{L W}=\mathbf{L S B}^{T}\left(\mathbf{B B}^{T}\right)^{-1}
$$

which is of the form

$$
\mathbf{L W}=\mathbf{R}
$$

with $\mathbf{R}$ known.

The Laplacian matrix always has rank . $f-1$ so it cannot be removed by premultiplying with " $\mathrm{L}^{-1}$ ". A solution is to require boundary conditions, i.e. that $\mathbf{W}$ is known (and is probably zero, a neutral pose) at the first and last frame, and then reorder $\mathbf{L W}=\mathbf{R}$ so that the known elements of $\mathbf{W}$ appear in the last two rows. This can be written as a block matrix system

$$
\left[\begin{array}{cc}
\mathbf{L} & \mathbf{L}^{u c} \\
\mathbf{L}^{c u} & \mathbf{C}
\end{array}\right]\left[\begin{array}{l}
\mathbf{W} \\
\mathbf{E}
\end{array}\right]=\left[\begin{array}{l}
\mathbf{R}^{1} \\
\mathbf{R}^{2}
\end{array}\right]
$$


with (beware) some renaming: $\mathbf{L}$ is now the $. g^{2}=(. f-2)^{2}$ upper left block of the full Laplacian matrix; $\mathbf{L}^{u c}$ are entries of the Laplacian that couple the unknown and constrained variables, $\mathbf{C}$ are entries of the Laplacian that couple the constrained variables, $\mathrm{W}$ now contains all but the two known rows of the previous $\mathbf{W}, \mathbf{E}$ contains the known ("edge") rows of $\mathbf{W}$, and $\mathbf{R}_{1}, \mathbf{R}_{2}$ split the previous $\mathbf{R}$ into the all-but-last-two and last two rows. The dimensions of this system are

$$
\left[\begin{array}{cc}
L_{. g, g} & L_{. g, 2}^{u c} \\
L_{2, . g}^{c u} & C_{2,2}^{c}
\end{array}\right]\left[\begin{array}{c}
W_{\cdot g, m} \\
E_{2, m}
\end{array}\right]=\left[\begin{array}{c}
R_{. g, m}^{1} \\
R_{2,, m}^{2}
\end{array}\right]
$$

Moving the known parts to the right hand side, we have

$$
\begin{aligned}
& \mathbf{L W}=\mathbf{R}^{1}-\mathbf{L}^{u c} \mathbf{E} \\
& \mathbf{L}^{c u} \mathbf{W}=\mathbf{R}^{2}-\mathbf{C E}
\end{aligned}
$$

and stack $\mathbf{L}$ over $\mathbf{L}^{c u}$ and the similarly for the right hand side,

$$
\left[\begin{array}{c}
\mathbf{L} \\
\mathbf{L}^{c u}
\end{array}\right][\mathbf{W}]=\left[\begin{array}{c}
\mathbf{R}^{1}-\mathbf{L}^{u c} \mathbf{E} \\
\mathbf{R}^{2}-\mathbf{C E}
\end{array}\right]
$$

which is an overdetermined system that can be solved for $\mathbf{W}$.

\subsection{Handling Regularization}

It is advisable to regularize the solution of any linear system. Rather than add a simple weight decay, it is possible to train a regularizer to penalize variations that are not present in some training data (this approach was used previously in Equation 4.7). The regularizer penalizes each weight in proportion to a corresponding standard deviation measured from the training data: $\left(\frac{w_{k}}{\sigma_{k}}\right)^{2}$. Applying the regularizer to all frames and adding to our objective, we have

$$
\min _{W} \operatorname{tr}(\mathbf{D W B}-\mathbf{D S})^{T}(\mathbf{D W B}-\mathbf{D S})+\lambda \sum_{k}^{. f} \sum_{b}\left(\frac{W_{k, b}}{\sigma_{b}}\right)^{2}
$$


We would like to re-express the regularization term in a form where it can easily appear in a linear system solve. To do this, first express $\mathbf{W}$ as a vector $\mathbf{w}=\operatorname{vec}(\mathbf{W})$.

The prior term $\sum_{k}^{f} \sum_{b}\left(\frac{W_{k, b}}{\sigma_{b}}\right)^{2}$ can be expressed as

$$
\mathbf{w}^{T} \boldsymbol{\Sigma}^{-T} \boldsymbol{\Sigma}^{-1} \mathbf{w}
$$

where $\Sigma$ is a diagonal matrix having $\sigma_{b}$ repeated along its diagonal.

The DWB term can be re-expressed as

$$
\mathbf{D W B} \Rightarrow\left(\mathbf{B}^{T} \otimes \mathbf{D}\right) \mathbf{w}
$$

where $\otimes$ is the Kronecker product.

Denote $\mathbf{d}=\operatorname{vec}(\mathbf{D S})$, and $\mathbf{J}=\left(\mathbf{B}^{T} \otimes \mathbf{D}\right)$. Then the overall objective is

$$
\min _{\mathbf{w}}\|\mathbf{J} \mathbf{w}-\mathbf{d}\|^{2}+\lambda\left\|\mathbf{\Sigma}^{-1} \mathbf{w}\right\|^{2}
$$

The $\mathbf{J}$ matrix is huge; it is of size $. v 3 \cdot f, . m \cdot f$, and contains $. v 3 \times . m$ copies of $\mathbf{D}$, each scaled by $B_{i j}^{T}$. However, $\mathbf{J}$ is sparse because $\mathbf{D}$ is sparse, and in fact the retargeting problem for a typical movie shot (of 3-5 seconds) can be solved at interactive rates on current machines [214].

\subsection{Chapter Summary}

This chapter motivated a "space-time" approach to the expression cloning problem, in which the entire temporal history of a source actor's motion is used in transferring that motion to a geometrically dissimilar target character. The particular formulation resembles a Poisson problem, but specialized for the case where the target is a blendshape model.

For an expression cloning problem where the source and target models are different but not "too different", this approach has some advantages over the popular expression cloning approach based on FACS (see section 3.5.3. For one, it does not require a FACS basis for the source performance. 
As mentioned in section 3.5.3. producing this basis can be an unnatural task for an actor. A second benefit is that it brings the power of Poisson editing (as introduced in [185] for image editing) to the problem.

On the other hand, the underlying principle that "the movement should be similar" excludes scenarios where the movement of the source and target models is (by design) not similar. This might easily arise when the target character is very different from a human. For example, perhaps a duck character might "smile" by moving its mouth corners backward rather than upward.

The material in this chapter was the author's contribution to the publication [214]. The general idea of a space-time approach to expression cloning deserves to be explored further, since it is likely to be the only approach that can produce temporal effects such as differing speech coarticulation between the source and target. Our particular formulation uses a least-squares matching between the source and target derivatives, which minimizes the largest differences at the expense of ignoring smaller changes. It may be interesting to consider using a 1-norm (sum of absolute value of differences). In addition, minimizing a "Sobelev" blend of position and gradient might have benefits. 


\section{Chapter 6}

\section{Exploring the Character of Face Space}

While the goal of this thesis is to develop practical algorithms to be used by artists, in any research it is worthwhile to briefly consider the problem from a general and abstract viewpoint. This chapter analyzes the "space of faces" from an abstract geometric point of view. While this investigation has intrinsic interest, it also underlies the choice of priors for any algorithms dealing with a full range of face shapes or expressions. The choice of prior is particularly relevant for highly underconstrained inverse problems such as the direct-manipulation editing developed in Chapter 4.

Since "face space" has been variously estimated as having anywhere from 40-100 dimensions we consider whether faces can be considered as drawn from a high-dimensional Gaussian. This assumption underlies the commonly utilized principal component models of faces, as well as algorithmic operations such as maximum a posteriori (MAP) inference.

We conclude that while the Gaussian assumption may be questionable, it is intuitions transferred from experience with low-dimensional Gaussians that are in fact more problematic. For example, the interior of the high-dimensional Gaussian density is virtually empty, so the mean face is in a sense not representative. We present evidence that assuming other- 
wise produces visibly poor results. A useful alternative view is to consider faces as lying near the surface of a (approximately) hyperellipsoidal shell.

While this chapter references linear models of the face for discussion purposes, Gaussian priors and MAP have been used in computing body motion as well [54].

\subsection{Introduction}

In computer vision and graphics research, facial expression and identity are commonly modeled as a high-dimensional vector space, often with a multidimensional Gaussian density. This choice of representation has associated algorithmic approaches such as linear interpolation and maximum a posteriori (MAP) solution of inverse problems.

In this chapter we argue several things: 1) the linear and Gaussian assumptions are not strictly correct. 2) existing research that starts from these assumptions has implicitly assumed a low dimensional setting. In high dimensions, common algorithmic approaches such as MAP may not be justified. 3) the problems resulting from these assumptions are not just hypothetical, but are visible in a practical computation, specifically interpolation of faces. Most importantly, we show that consideration of these factors can result in an algorithm with visibly improved results.

\subsection{Linear models}

The faces of realistic computer characters in movies are most often generated using the "blendshape" representation [131, 11, 212, 142]. This is a linear representation of the form $\mathbf{f}=\mathbf{B w}$, where $\mathbf{B}$ is a linear but non-orthogonal basis having semantic meaning. In computer vision, approaches such as active appearance models (AAM) [69] and morphable models [33] use an orthogonal basis generated by principal component 

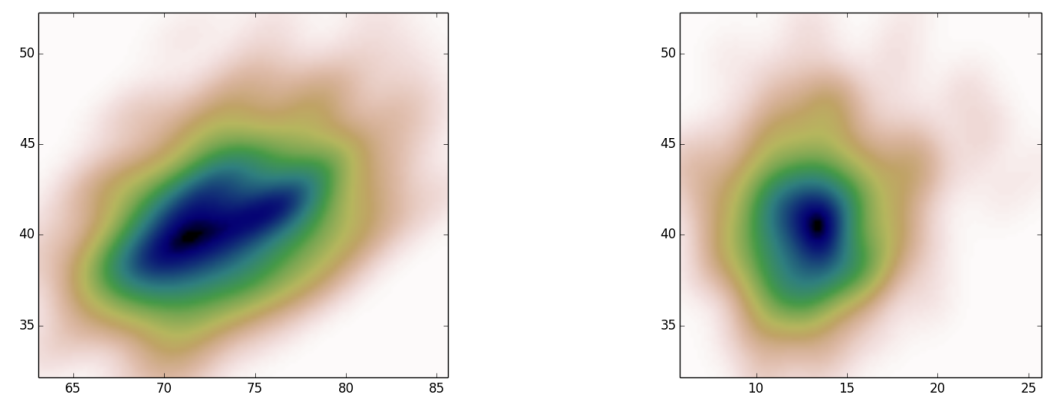

Figure 6.1: Face proportions are not strictly Gaussian. Kernel density plots of (left) the distance between the eyes versus the width of the mouth, (right) the width of the mouth versus the height of the mouth, measured from a database of 359 faces.

analysis (PCA), and assume the multidimensional Gaussian prior. Bilinear (tensor) face models have also been proposed [240]. Psychological research has also employed such linear models with a multivariate Gaussian prior [238].

PCA assumes that the data is jointly Gaussian, in that the PCA basis vectors are the eigenvectors of a covariance matrix that does not capture any non-Gaussian statistics. The Gaussian assumption leads to a frequently employed prior or regularizer of the form $\mathbf{c}^{T} \boldsymbol{\Lambda}^{-1} \mathbf{c}$ where $\mathbf{c}$ is the vector of PCA coefficients and $\Lambda$ is the diagonal matrix of eigenvalues. The eigenvalues are variances of the data in the directions of the eigenvectors. The Gaussian assumption also naturally leads to the MAP approach to regularising inverse problems. This approach selects model parameters $M$ as the mode of the posterior $P(D \mid M) P(M)$ given data $D$. With a Gaussian model the posterior also has a Gaussian form.

The appropriate number of dimensions for a linear facial model of expression or identity has been variously estimated to be in the range 40-100 $[160,184,156]$. High quality blendshape facial models used in movie visual effects sometimes have on the order of 100 dimensions [131]. The 


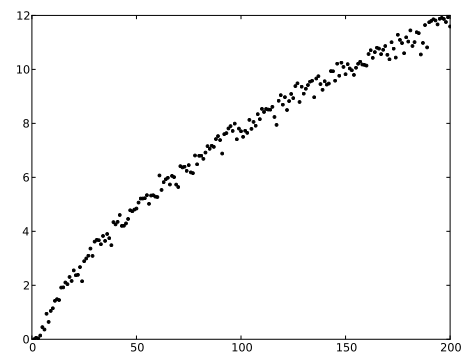

Figure 6.2: The closest distance to the mean among 1000 unit-variance multidimensional Gaussian random variables (vertical axis) as a function of the dimension (horizontal axis). In 100 dimensions every point in this simulation is more than six standard deviations from the mean.

main character in the 3D animated movie Toy Story had 212 parameters controlling the head [102].

In figure 6.1 we show that the common multidimensional Gaussian assumption is not strictly accurate. This figure shows a kernel density plot of several simple measurements of facial proportions measured from 359 selected photographs from the facial database [186]. It is also somewhat obvious that a linear model is not entirely appropriate for facial expression. For example, the motion of the jaw has a clear rotational component. On the other hand, the widespread use of the blendshape representation in movies (albeit sometimes with nonlinear correction terms [212]) is an argument that linear models suffice even if they are not strictly accurate. It is less clear whether a vector space model of facial identity is appropriate, or if a (nonlinear) manifold assumption would be more accurate. While these comments call into question the linear and Gaussian assumptions, existing research does not indicate whether these objections are important in practical computations. 


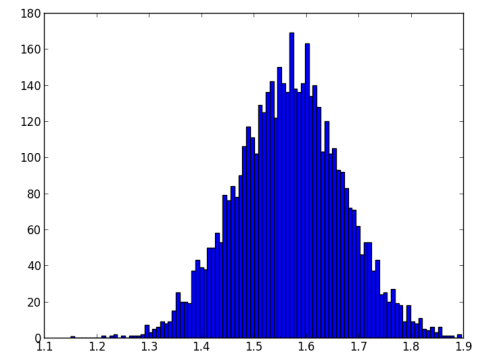

Figure 6.3: Histogram of the angles between all pairs of 100 randomly chosen isotropic Gaussian random variables in 100 dimensions. The angles cluster around $\pi / 2$ : in high dimensions, most data are nearly orthogonal.

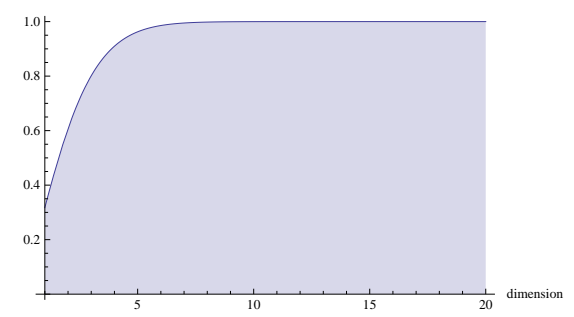

Figure 6.4: Probability that a sample from a unit variance Gaussian is outside the unit hypersphere for various dimensions. 


\subsection{High-dimensional Phenomena}

High dimensional data is generally subject to a collection of nonintuitive phenomena collectively known as the "curse of dimensionality" [243]. Examples of such phenomena are that a) in high dimensions, "all data is far away" with high probability (Figure 6.2), b) randomly chosen vectors are nearly orthogonal (Figure 6.3), and c) the probability mass of the data is overwhelmingly located near the surface of the hypervolume, with the interior of the volume essentially empty (Figs. 6.5, 6.8).

Current face computation approaches generally overlook these phenomena. A notable exception is [183], who described the following apparent paradox: the squared Mahalanobis distance $\mathbf{c}^{T} \boldsymbol{\Lambda}^{-1} \mathbf{c}$ follows a $\chi^{2}$ distribution with $n$ degrees of freedom, since it is the sum of independent, identically distributed (i.i.d.) squared Gaussian variables of variance $\frac{c_{i}^{2}}{\lambda_{i}}$. The expectation of this distribution for $d$ dimensions is $d$, thus we expect the length of the standardized squared coefficient vector of a typical face to be $d$. However under the multidimensional Gaussian model, the face at the origin (the mean face) is the most probable, and the length of its squared coefficient vector is zero.

[183] also state a hypothesis that faces should lie on the shell of a hyperellipsoid dictated by the squared coefficient length. The resolution to the apparent paradox is simply that it is the difference between the variance and mean. A zero-mean random variable can (and typically does!) have a nonzero variance. Randomly sampling from a multidimensional Gaussian will generate a sequence of samples that have both the expected mean and variance of course.

\subsection{The High-Dimensional Gaussian Prior}

Next we will verify the statement that high dimensional data is concentrated overwhelmingly near the surface of the hypervolume. In the case 


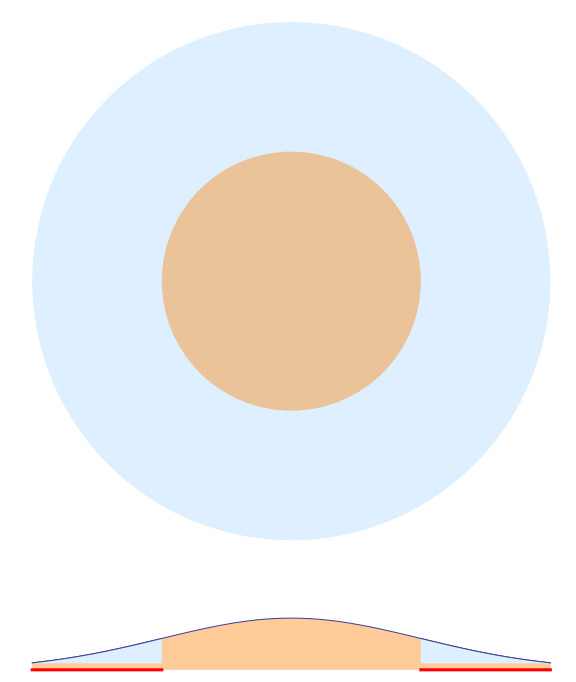

Figure 6.5: Bottom: schematic one dimensional Gaussian distribution, with the area between one and two deviations indicated in red. This interval is equal to that of the unit radius. Top: In two dimensions, the area between one and two standard deviations (light blue) is relatively larger than the area of the unit standard deviation disc (light orange). Figure is best viewed in the electronic version of this document.
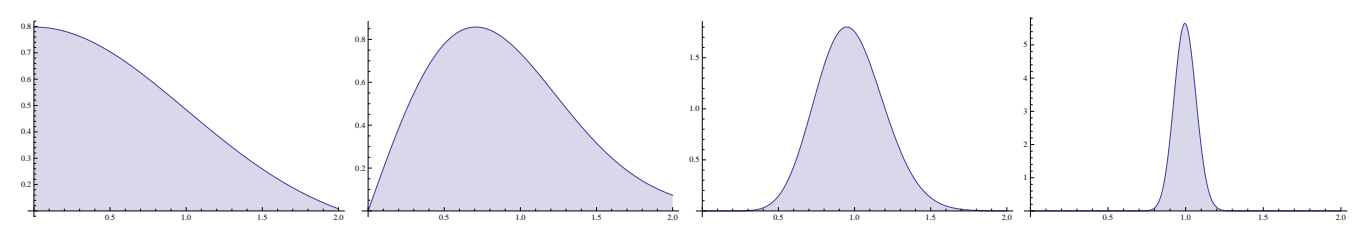

Figure 6.6: The radially integrated Gaussian $N\left(0, \mathbf{I}_{n}\right)$ in various dimensions. Each subfigure shows the radially integrated Gaussian profile $S_{d-1}(r) G(r)$ (vertical axis) plotted in units of $\sqrt{d}$ (horizontal axis). From left to right: 1, 2, 10, and 100 dimensions. In high dimensions the probability concentrates in a shell centered at radius $\sqrt{d}$. 
of a uniformly distributed random variable in a hypercube, this is easy to see. Consider a unit hypercube in $d$ dimensions, that encloses a smaller hypercube of side $1-\epsilon$. As $d \rightarrow \infty$, the volume of the enclosed hypercube is $(1-\epsilon)^{d} \rightarrow 0$.

The fact that the multivariate Gaussian is a heavy tailed distribution in high dimensions is less obvious. For example, [238] states, "even for a face space of high dimensionality, the assumption of a multivariate normal distribution means that... There will be many typical faces that will be located relatively close to the center". However this phenomenon is at least intuitively suggested by comparing the one- and two-dimensional Gaussian distributions (Figure 6.5). In one dimension, the "volume" of the interval between one and two standard deviations is equal to the radius of the unit interval. In two dimensions the area of the annulus between one and two standard deviations is relatively larger than the area of the unit disc. In higher dimensions the trend continues, with the available volume overwhelmingly concentrated near the outside.

Discussion of the multivariate Gaussian is simplified by a "whitening" transformation $c_{i} \rightarrow c_{i} / \sqrt{\lambda_{i}}$ from the original hyperellipsoidal density to an isotropic density. We can also consider a unit-variance density without loss of generality. In this case the probability that a point is within a hypersphere of radius $r$ is proportional to

$$
\int_{0}^{r} S_{d-1}(r) G(r)=\frac{2 \pi^{d / 2}}{\Gamma(d / 2)} \int_{0}^{r} r^{d-1} G(r) d r
$$

where $d$ is the dimension, $G(r)=\frac{1}{\sqrt{(2 \pi)^{d}}} \exp ^{-r^{2} / 2}$ is the isotropic unit variance Gaussian density function, $S_{d-1}(r)=\frac{2 \pi^{d / 2} r^{d-1}}{\Gamma(d / 2)}$ is the "surface area" of the $d$-hypersphere, and $\Gamma$ is the Gamma function. This can be used to plot the tail probability that a point lies outside the unit hypersphere in various dimensions (Figure 6.4). While in one dimension the majority of the probability mass is within the unit interval, in 100 dimensions the probability that a point is outside the unit hypersphere is 1 . to within machine precision! It may be worth contrasting the mode of the high-dimensional 
Gaussian with the Dirac delta generalised function familiar in signal processing [42]. The delta function has zero width but unit volume when integrated over. In contrast, the high-dimensional Gaussian has nonzero width near the origin, but negligible volume.

High dimensional data can also be tightly concentrated in a shell of relatively narrow thickness. In the case of the multi-dimensional Gaussian, the majority of its mass is concentrated within a shell centered at radius $\sqrt{d}$. Figure 6.6 plots the radially integrated unit variance Gaussian profile $S_{d-1}(r) G(r)$ relative to the distance $\sqrt{d}$ (i.e. with a change of variable $r \rightarrow r \sqrt{d}$ ). The data is concentrated increasingly around $\sqrt{d}$ (relative to the distance $\sqrt{d}$ itself) in high dimensions.

The observations collected above lead to the remarkable conclusion that algorithms such as MAP may be nonsensical in high dimensions! This conclusion is not widely known in the computer vision and graphics community, where MAP is commonly used for face computations with models having 10-100 dimensions.1 However, our conclusion is supported in [151], where Mackay states "probability density maxima often have very little associated probability mass even though the value of the probability density there may be immense, because they have so little associated volume... the locations of probability density maxima in many dimensions are generally misleading and irrelevant. Probability densities should only be maximized if there is good reason to believe that the location of the maximum conveys useful information about the whole distribution."

\footnotetext{
${ }^{1}$ In fact many results in statistics focus on the case where increasing amounts of data are available, i.e. $n / d \rightarrow \infty$ with $n$ the number of data points. In our problem we may have $n / d$ finite and small, as in the case of a face model with several hundred training examples, each with 100 degrees of freedom.
} 

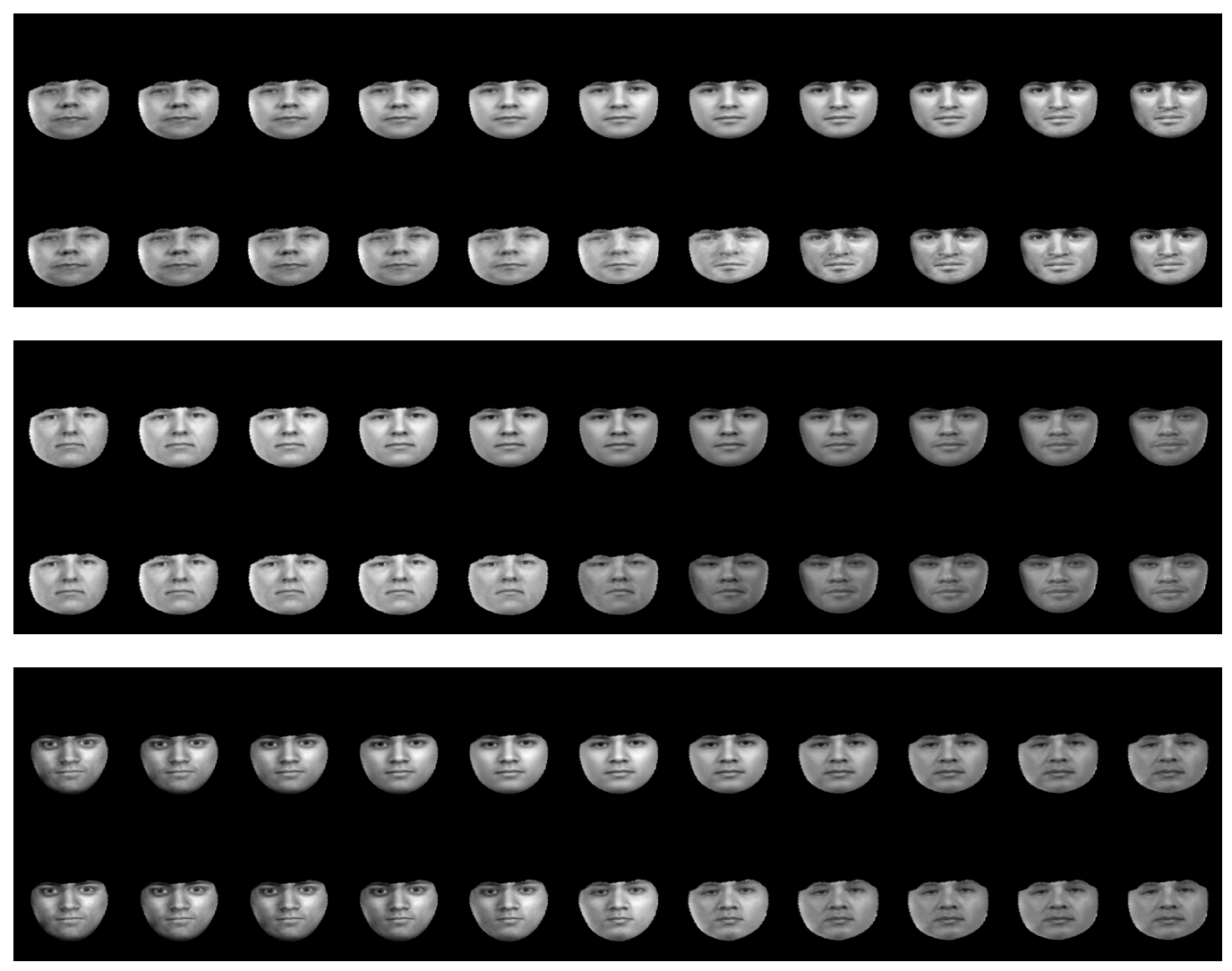

Figure 6.7: Interpolating between a randomly chosen face (left column) and a second face (right column) nearly on the opposite side of the hyperellipse of coefficients. Top row of each image: linear interpolation of coefficients. The middle images lack distinctiveness. Bottom row of each image: interpolating "around the hyperellipse". Detail is preserved throughout the interpolation. 


\subsection{Example Computation: Interpolating in Face Space}

Figure 6.7 contrasts two approaches to interpolating facial identity. The images are not photographs but are synthesized with an AAM [162]. The face on the far left is generated from a coefficient vector $\mathbf{c}_{l}$ sampled from a multivariate Gaussian with the appropriate variances (eigenvalues). The face on the far right is also randomly chosen, but its coefficient vector $\mathbf{c}_{r}$ is modified to constrain it to having a specified inner product $\left\langle\mathbf{c}_{l}, \mathbf{c}_{r}\right\rangle_{\Lambda^{-1}}=$ -0.8 so as to place it on the opposite side of the coefficient volume. The inner product uses the inverse eigenvalue-weighted norm $\left\langle\mathbf{c}_{l}, \mathbf{c}_{r}\right\rangle_{\boldsymbol{\Lambda}^{-1}}=$ $\mathbf{c}_{l}^{T} \boldsymbol{\Lambda}^{-1} \mathbf{c}_{r}$. The dimensionality of the space (length of the coefficient vector) is 181 .

The top rows in figure 6.7 shows linear interpolation through the Gaussian coefficient space. The midpoint of this interpolation passes closer to the center (mean) face than either end. This results in a somewhat "ghostly" face that lacks detail. The linear interpolation also has the undesired result that (for example) interpolating from a person of age 40 to a person of age 45 might pass through an intermediate face of apparent age 25 , if that is the mean age of the database underlying the AAM.

In the lower panels of figure 6.7 we interpolate "around" a hyperellipsoidal shell in the coefficient space rather than across the volume. Given initial and final coefficient vectors $\mathbf{c}_{l}, \mathbf{c}_{r}$, at each step a coefficient vector is generated that interpolates the norm of these vectors (although in fact the difference in norm is expected to be small due to phenomena mentioned above). This interpolation remains inside the high probability shell of the hyperGaussian and generates distinctive faces throughout the interpolation. 
Figure 6.8: In this schematic illustration the point along the constraint (dark line) that has the highest probability is the red point. In high dimensions however, the interior of the Gaussian is empty and the probability mass is concentrated toward the outside.

\subsection{Chapter Summary}

This chapter describes known high-dimensional phenomena that call into question common assumptions underlying much computer vision, graphics, and psychological research on face computation. In particular, we question approaches that assume that typical faces lie in the interior of a high-dimensional Gaussian density (Figure 6.8). The issue is not due to a bi- or multimodal distribution (as with a combined distribution containing both women and men) but rather is a consequence of high dimensionality. These objections are not merely hypothetical, but are visible in a simple face computation. Our conclusion highlights the need to develop new algorithms that address the intrinsically high-dimensional nature of facial identity and expression. 


\section{Part II}

\section{Nonlinear Models}

Linear facial models have been widely and successfully employed in practice, despite being a simple approximation of reality. In contrast, a linear model for body movement is not viable - the motion of the body limbs is mostly rotational, and direct use of a linear basis would result in serious artifacts such as limbs that change in length. In this second part of the thesis we analyze the case of example-based deformation for body motion, and describe how this can be successfully formulated as a highdimensional nonparametric regression problem. 


\section{Chapter 7}

\section{The Example-Based Approach to Creature Skinning}

The previous chapters described linear models for faces and introduced several advanced editing approaches for such models. While it is obvious that the linear model is a simplification, it has been successfully used in practice. In contrast, the body of an animal or human exhibits significant rotational motion that cannot easily be described with a linear model.

This chapter surveys nonlinear example-based approaches that are suitable for describing the skin surface deformation of a moving articulated character. One such approach, "pose space deformation" (PSD), was introduced in the author's publication [139]. It allows the desired shapes of a creature's body in various poses to be directly specified by the artist, either by sculpting or scanning. These shapes are then interpolated as a function of pose, resulting in animation that reproduces the specified training poses.

The novelty of this approach is that:

1. Animation has traditionally been considered as "shape as a function of time". PSD instead views animation as "shape as a function of pose" (Fig. 7.1). The advantage of this alternate view is evident even before considering specific algorithms: the motion of an articulated 
figure often passes near the same poses multiple times (a walk cycle being the prototypical example). By considering the space of poses rather than motion over time, each pose can be handled just once, rather than every time it arises.

2. It introduces an "example based" approach to the skinning problem, in which the desired shape of the skin is directly specified either by sculpting or digitizing examples.

The example-based approach is in contrast to traditional "rigging" approaches in which the basic skin deformation is specified by a simple algorithm (such as linear blend skinning), and additional effects are approximated by simple mathematical expressions [108, 157, 173]. As an example of these "shallow" algorithmic approaches, a muscle bulge shape might be triggered as a Gaussian function of the elbow rotation. These rigging approaches have clear disadvantages. Firstly, the desired deformation is difficult to obtain with algorithms and simple expressions. Secondly, the process of adjusting the parameters is time consuming.

This chapter together with the following chapter 8 extend [139] in several ways:

- We discuss the PSD algorithm in its proper context as a nonparametric regression technique. This leads to consideration of issues such as regularization and the curse of dimensionality.

- We survey work that builds on and greatly improves [139], for example by significantly reducing the number of training examples that are required [120], and by formulating the domain of the deformation in terms of geodesic distance on the surface of a mesh [200].

- We consider cases where the data has duplicate items, poor spacing, or outliers. 
- We consider the choice of the radial basis kernel function.

- We describe the derivation of the radial basis kernel.

The chapter also collects experience with these example-based skinning algorithms, describes a variety of applications, and informs the design choices for new implementations.

\subsection{Introduction}

Example-based skinning approaches generate skinning and other effects by interpolating a set of sculpted or scanned examples of the desired deformation, where the interpolation happens in a "pose space" defined by the joint angles and other degrees of freedom of the creature rig.

The example-based approaches are distinguished by two main ideas:

1. Skinning usually specifies the deformed position of a vertex using a short algorithm, such as linear blend skinning or dual quaternion skinning. In contrast, example based approaches interpolate a set of examples of the desired deformation.

2. Traditionally animation has been abstractly considered as a function of time, i.e.

$$
\text { shape }=f(\text { time })
$$

In computer graphics we are also familiar with surface interpolation, where

$$
\text { shape }=f(\text { space }) \text {. }
$$

In contrast, example-based approaches consider

$$
\text { shape }=f(\text { pose })
$$

where "pose" is the pose of the underlying skeleton (usually) or other non-spatial parameters. 


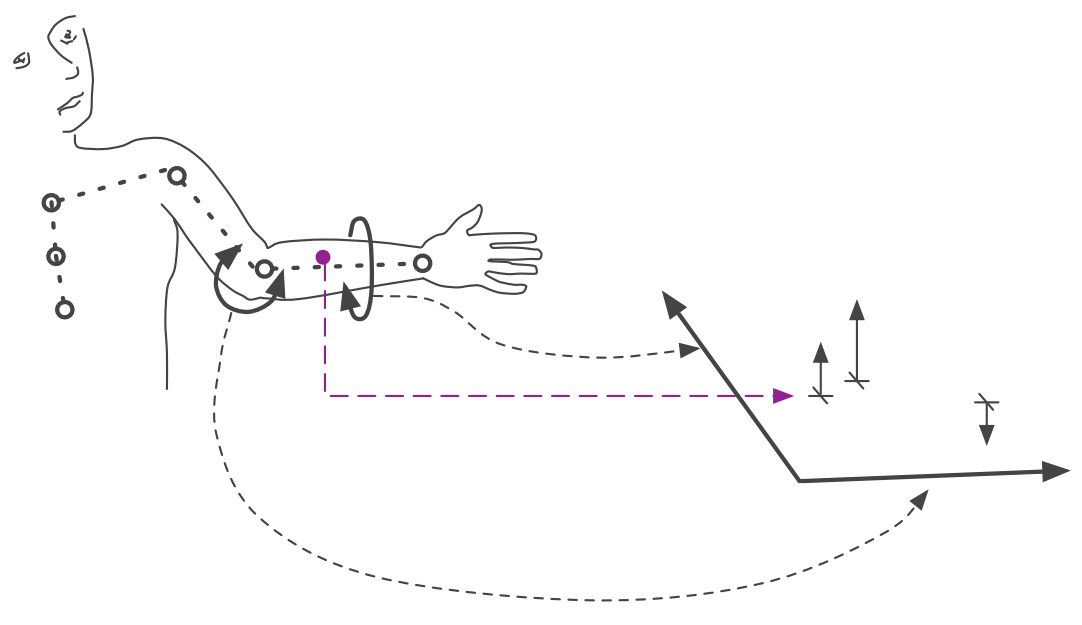

Figure 7.1: Scattered interpolation of a creature's skin as a function of skeletal pose. Left, A point on the forearm (red in the electronic version) moves as a function of two degrees of freedom (dofs): the elbow rotation and the forearm twist. Example shapes describing the deformation of this point are interpolated in an abstract 2-dof pose space (right).

The example-based skinning (EBS) approach has evident advantages and disadvantages:

- The desired deformation is directly sculpted, so the artist gets exactly what s/he wants. Algorithmic skinning approaches generally require specifying parameters that only indirectly relate to the desired shape, such as the weights on various transforms.

- The range of possible deformed shapes is much larger than is achievable with current algorithmic skinning algorithms. For example, it is possible to sculpt a shape that includes a bulging muscle and extruding veins. The example-based approach permits a progressive 
refinement strategy, whereby additional shapes are added as needed to obtain the desired quality.

- Among disadvantages of EBS, it requires considering higher-dimensional pose spaces. For example, the number of rotations influencing the shoulder region is probably more than three, and the number of bones influencing the neck/trunk area of an animal such as an elephant might be 10 or more. EBS requires computation proportional to the number of examples. This is likely higher than the amount of computation required for algorithmic skinning.

- Lastly, EBS can require some awareness of the numerical considerations that arise in interpolation and data fitting. These include regularization and the curse of dimensionality.

Comparing an example-based algorithm such as PSD to Blendshapes is instructive, and a loose comparison with manifold learning ideas [202, 230] is possible. In blendshapes and many other geometric interpolation schemes the "space" is defined by the geometric dimensionality of the model, e.g. $\Re^{3 n}$ for a model with $n$ control vertices. In PSD, the space is analogous to a manifold within the ambient geometric space, defined by a small number parameters selected by the artist. The examples are placed on this manifold at desired locations.

Example-based and algorithmic skinning approaches are not mutually exclusive. On the contrary, example-based skinning is usually implemented as a correction on top of algorithmic skinning.

The deformed postion of a single vertex $\hat{\mathbf{p}}$ at a pose $\mathbf{p}$ will be interpolated from the corresponding positions specified at example poses $\mathbf{p}_{k}$. Preferably, an EBS system should allow the artist to specify a desired shape at any location in the pose space as desired (Figure 7.1). Since these locations have no regularity, this requires the use of a scattered interpolation algorithm. 
This section of the thesis will first describe some of the example-based algorithms, while leaving the underlying scattered interpolation method as an unspecified "black box". Next, several examples and applications of EBS methods will be mentioned. Following that, we will review scattered interpolation methods that have been used for EBS. Lastly, numerical considerations will be mentioned. Skinning is a core component of the broader subject of "rigging". For recent surveys of character and facial rigging, see [157, 173].

\subsection{EBS Algorthms}

\subsubsection{Pose Space Deformation}
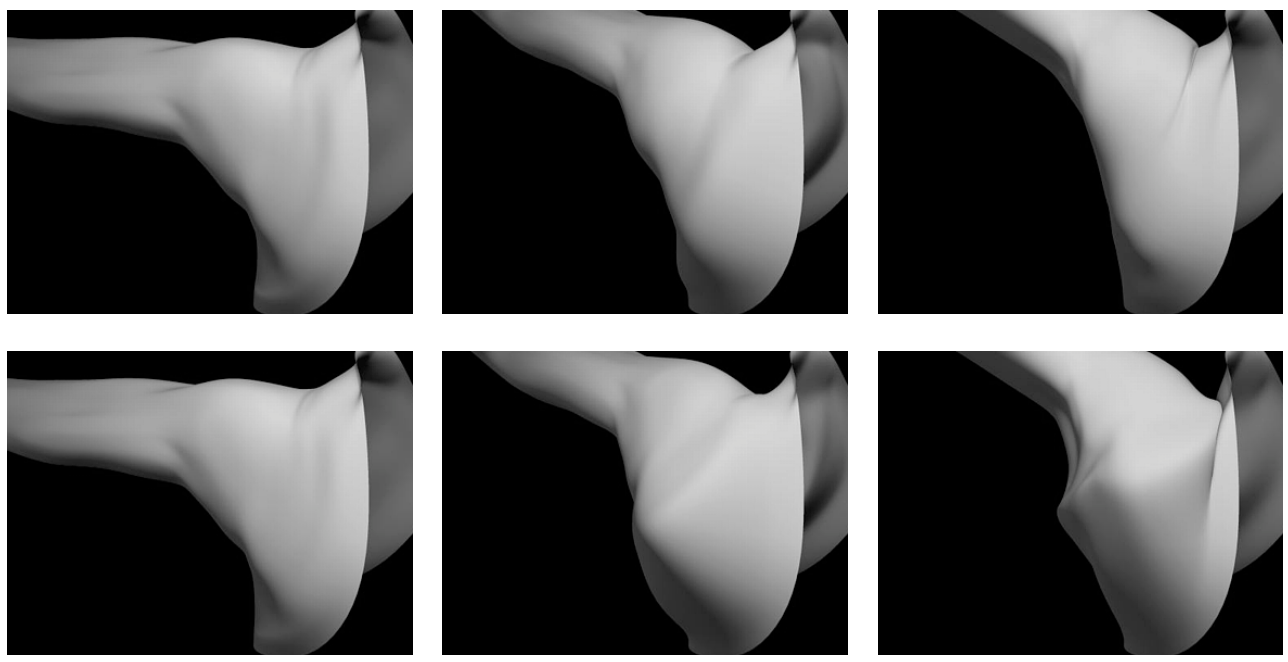

Figure 7.2: Comparison of PSD and LBS on an animating shoulder. Top, PSD using only two sculpted poses. Bottom, LBS. The shoulder area is especially problematic for LBS due to the large range of rotational movement.

In the pose-space deformation (PSD) algorithm ([139]; Figures 7.1, 7.2. 7.3) the artist moves the underlying skeleton to particular poses and sculpts 

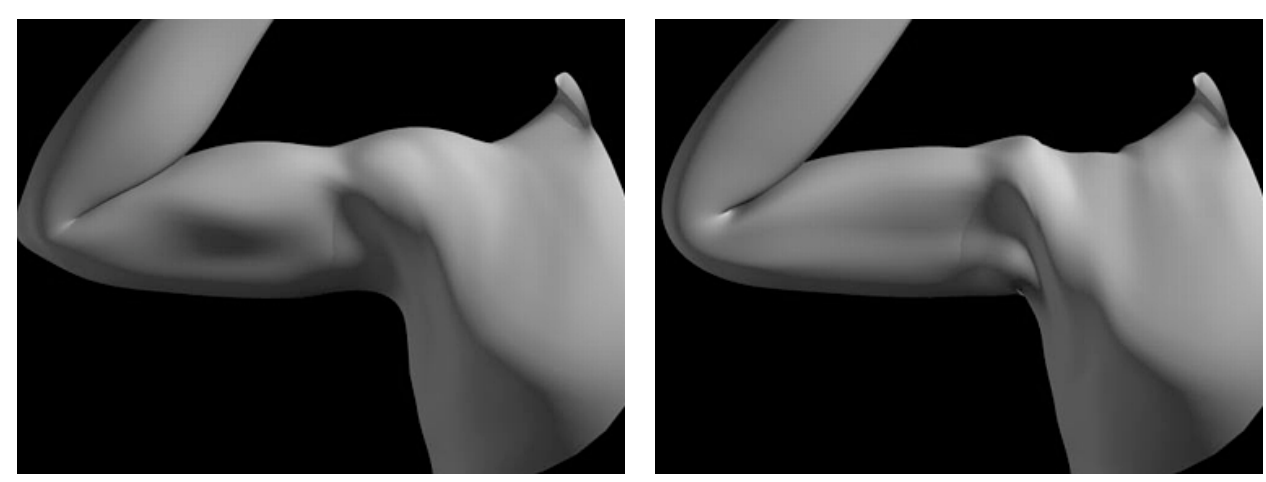

Figure 7.3: Comparison of PSD (at left) and LBS on the extreme pose of an elbow.

examples of the desired shape at those poses. The sculpts are saved and associated with the corresponding pose. The PSD system interpolates these examples as a function of pose.

The pose space is a set of degrees of freedom of the rig that vary between the example shapes, and a particular pose is a set of particular values of these degrees of freedom (not to be confused with the shape that the model has at that pose). Typically, the pose space is the set of joint angles of the skeleton that vary across the given shapes. If the sculpted examples are of the shoulder region, the "pose space" would consist of the joint rotations of the bones that plausibly affect the shoulder. More generally, however, the pose space can include other attributes. For example it might include a degree of freedom describing "exertion", so that muscles can bulge appropriately when the body is carrying weight. [222] termed these degrees of freedom "adjectives."

The interpolation of the examples has the pose space as its domain, and 3D space is the range of the interpolation. For example, a point near the neck may be affected by three bones. A joint between two such bones might be described by two relative angles. The three-bone configuration has two internal joints, so the pose space in this case has four dimensions. The interpolation is then from a 4-dimensional pose space to 3D (geome- 
try) space $\left(\Re^{4} \rightarrow \Re^{3}\right)$.

Since the examples can be created at arbitrary locations, this is a scattered interpolation problem (Figure 7.1). In machine learning terms it can also be considered as an instance of non-parametric regression ("nonparametric" refers to a model whose complexity increases with the available data, as opposed to a parametric model such as a Gaussian that has a fixed number of parameters (e.g. mean, variance) regardless of the amount of data). In the machine learning terminology the collected set of example shapes may be termed "training" shapes.

The example based approach is extremely simple (although it can be occasionally confusing to mentally switch between thinking about 3D space and the higher-dimensional pose space!)

Particular EBS algorithms differ in the way they handle several issues:

Layering. Typically the PSD is sculpted as a correction or offset from either the rigid transformation of the surface or an underlying skinning system such as linear blend skinning (LBS) [139], spherical skinning [257], or dual-quaternion skinning [166, 113]. Additional corrections can be layered as desired, providing an iterative refinement workflow.

Inverting the skinning operation. It is preferable if example-based skinning operates in the local coordinate frame, so that it can focus on learning the required deformation and ignore learning the rigid transform. In the case where PSD is layered on top of a skinning algorithm, this means that the PSD correction should be "pushed through" the skinning operation. That is, instead of deforming vertices as

$$
\hat{\mathbf{p}}=P S D(S K I N(\mathbf{p}))
$$

we want

$$
\hat{\mathbf{p}}=S K I N(P S D(\mathbf{p}))
$$


This requires inverting the effect of the skinning operation [222, 119, 256], in order to find a modified example that produces the same result when skinning is applied, as the original sculpted result produced when applied after skinning. This will be termed blending in the rest pose.

In the case of linear blend skinning this inverse can be calculated. However, when implementing a PSD system inside a larger package such as Maya, the existing skinning and rigging operations may be more complicated algorithms or proprietary "black box" deformers. In these production situations [256] proposed the use of derivative-free optimization to invert an arbitrary existing skinning rig.

The following three issues are distinct but related:

- Per-shape or per-vertex interpolation.

Shape-by-example [222] used cardinal interpolation ${ }^{1]}$ of the examples, with the same weights applied to all vertices of the example meshes (the blending is done in the rest pose space). Other methods have used differing interpolation at each vertex - this is required in the case of weighted pose space deformation.

\section{- Local or global interpolation in the pose space.}

All the degrees of freedom of the model can be concatenated to form a single global pose space. This presents problems however: consider a point on the fore-arm, as in Figure 7.1. If the pose space also contains the dofs of the legs, then moving the legs will affect the relative distance of the current pose from the poses that involve arm motion. In concept this could be addressed by adding examples with the legs in all possible shapes for every desired shape of the arm.

This is an instance of the "curse of dimensionality" (section A.7) the needed amount of training data rises exponentially with increas-

\footnotetext{
${ }^{1}$ In cardinal interpolation the $j$ the data point is added with a weight function $b_{j}$ having the property $b_{j}\left(\mathbf{x}_{i}\right)=\delta_{i j}$, i.e. the function is one at the location $\mathbf{x}_{j}$ of the $j$ th data point and zero at all the other data locations.
} 
ing dimension. The original PSD algorithm avoided the problem by using separate per-vertex interpolation, relying on spline interpolation to avoid any discontinuities. The weighted pose space deformation algorithm introduced a better approach, in which irrelevant dofs are smoothly attenuated (according to the underlying skinning weights) when calculating the distance in pose space. This results in a collection of smoothly coupled local regression problems, thereby addressing the curse of dimensionality without introducing discontinuities.

\section{- Interpolation algorithm.}

Independent of whether the interpolation is per-vertex or per-shape, or local or global in the pose space, different scattered interpolation algorithms can be chosen. Approaches that have been employed include linear interpolation, natural neighbor interpolation, and radial basis interpolation with several types of kernels. These possibilities are reviewed in section 8.1 .

\subsubsection{Shape by Example}

Shape-by-Example is a technique presented by Sloan, Rose, and Cohen at the 2001 I3D conference [222]. Their technique has some broad similarities to PSD. It differs in that the interpolation is formulated as a cardinal interpolation of the examples in the pose space.

In this scheme, for $n$ example shapes there are $n$ separate interpolators, with the $k$ th interpolator using data that is 1 at the $k$ training pose and 0 at all the other poses. These are then simply used as weights in a shape interpolation, albeit one that happens in the rest pose as described above.

The use of cardinal interpolation has several advantages. It uses a single global pose space for all examples, which is easier to think about. It also provides considerable efficiency if separate per-vertex interpolation effects are not required. 

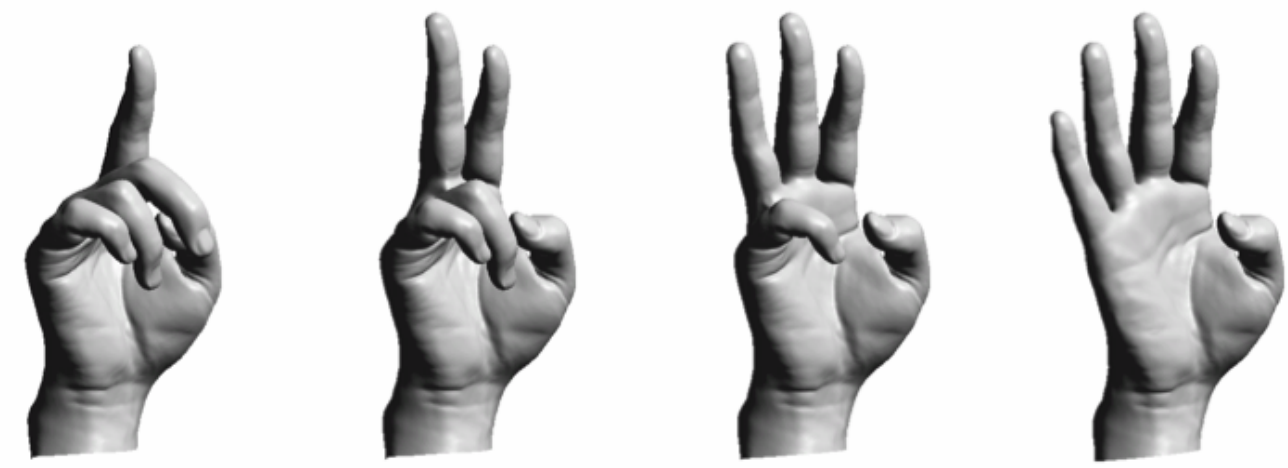

Figure 7.4: Hand poses synthesized using weighted pose space deformation (image from [120] (C)Tsuneya Kurihara).

Another innovation in this work was the concept of pseudo-examples. In this approach, the artist finds an acceptable interpolated shape situated at the wrong location in the abstract pose space. Rather than sculpt new examples, the interpolated shape is simply moved to a new location in the pose space. The result is that the pose space itself is reshaped. This can be desirable if, for example, a particular interpolation begins and ends with the right shape, but interpolates too quickly or slowly.

\subsubsection{Weighted Pose Space Deformation}

Kurihara and Miyata [120] introduced the weighted pose-space deformation (WPSD) algorithm. The WPSD algorithm solves the curse of dimensionality to some extent. It greatly reduces the number of training examples required, particularly for complex regions such as the hand that may have as many as 40 parameters or more. This is accomplished by formulating the interpolation as a set of separate but softly-coupled interpolations, one per vertex. The skinning weights are used to determine a per-vertex dis- 
tance between poses,

$$
d_{j}\left(\mathbf{p}_{a}, \mathbf{p}_{b}\right)=\sqrt{\sum_{k}^{n} w_{j, k}\left(\mathbf{p}_{a, k}-\mathbf{p}_{b, k}\right)^{2}}
$$

where $w_{j, k}$ are the skinning weights for the $k$ th degree of freedom for the $j$ th vertex, and $\mathbf{p}_{a, k}$ denotes the $k$ th component of location $\mathbf{p}_{a}$ in the pose space.

The example shapes in [120] were captured using a medical scan of one of the authors' hands, resulting in very plausible and detailed shapes (Fig. 7.4). The video accompanying the paper shows that the interpolation of these shapes as the fingers move is also very realistic.

Kurihara and Miyata used the cardinal interpolation scheme from [222], in combination with normalized radial basis functions. The RBF matrix is based on the distance between the poses (in pose space) and so has to be formed and inverted only once.

\subsubsection{Context-Aware Skeletal Shape Deformation}

[245] might be summarized as being a combination of deformation transfer [226] and weighted pose space deformation [120]. WPSD is used to blend triangles of the examples in the rest space. These triangles are then transformed by the skeleton's rigid motion using a approach inspired by deformation transfer. Specifically, the various bone transform affecting a particular triangle are blended (with log-quaternion blending of the joint rotations). The final position of each triangle is obtained by solving a Poisson problem involving the deformation gradient (Jacobian) of each triangle [40]. In an initial step the skinning weights are computed automatically using a harmonic cardinal interpolation of regions that are marked by the artist as moving rigidly with particular joints. 


\subsection{EBS Applications}

\subsubsection{Skeleton-Driven Deformation}

An articulated model such as a human will typically have a number of different deformation subspaces, each with one or several deformations; the deformations in different subspaces may overlap spatially e.g. to simulate the influence of different muscles. The deformations needed for an elbow, for example, will be interpolated in the one-dimensional subspace defined by the elbow joint angle. Deformations in a shoulder area will need to consider two or more degrees of freedom. The neck/chest/leg blend area of many quadrupeds is a more complex case - the motion of the skin surface in these regions may depend on the relative configuration of several leg bones as well as the rib cage and possibly the neck region of the spine. The example-based approach handles all these cases simply and uniformly.

Figures 7.2 and 7.3 show PSD and LBS algorithms in action on human elbow and shoulder regions.

\subsubsection{Secondary Animation}

Additional "dimensions" of deformation can be created by adding a new parameter and associating additional poses with the movement of this parameter. For example, a limb can be modeled in a particular pose both in an unloaded state and with muscles sculpted to express carrying a heavy load. The 'heavy' pose can be associated with the 'on' state of an abstract parameter; light and heavy loads can then be controlled by flipping this switch.

\subsubsection{Using PSD to "Reparameterize" an Existing Rig}

PSD can also be used to control parameters of a lower level "rig", rather than directly controlling geometry. It has often been employed this way in practice [127, 117]. 


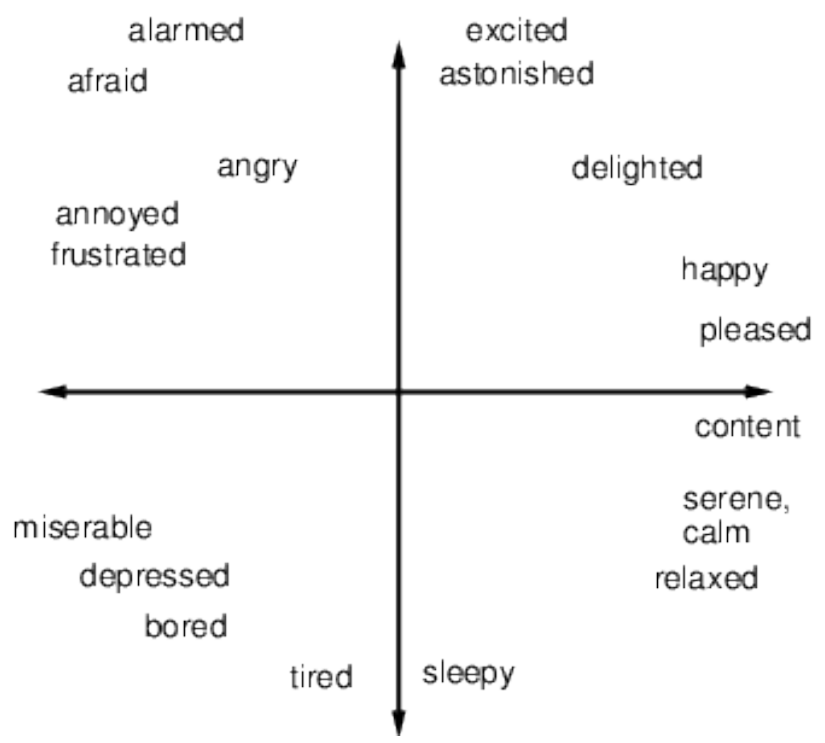

Figure 7.5: Schematic diagram of emotion space obtained by multidimensional scaling from pairwise similarity ratings, simplified from [203].

In this approach, the artist invents a new space for interpolation, with associated parameters that serve as controls. The underlying interpolation maps these visible parameters to the parameters of the underlying system, which are typically lower level or less convenient. The desired control scheme is specified simply by providing examples of the desired mapping.

\subsubsection{Facial Animation}

Disney introduced the use of PSD for facial animation [127, 117] (also [126]).

Decoupling parameters from examples. An advantage of example-based approaches for facial animation is that it decouples the control parameters from the location of the examples. In blendshape animation, each blend- 
shape target defines a control parameter. Abstractly, the examples (targets) are situated at some of the vertices of a hypercube (Figure 3.5). In an example-based approach, the artist defines a set of control parameters as desired, and then can place the example shapes anywhere in the space defined by these parameters (Figure 3.11). The number of example shapes does not need to be the same as the number of parameters.

As a simple example, one could take an emotion space identified in psychological research as the pose space. [203] performed multidimensional scaling (MDS) on surveys of pairwise similarity ratings between various facial expressions involving emotion, and identified two axes that can be interpreted as happy-sad and low-high energy. In this space, for example, "content" is low-energy but happy, whereas "bored" is low-energy but less happy. An emotional face model could be constructed by sculpting any number of facial expressions representing various emotions, and then placing them at the appropriate point in the space (Figure 7.5).

This simple example shows how using a pose space can greatly reduce the number of parameters that the artist needs to control. On the other hand, expressive facial animation is a high-dimensional problem even if the most economical parameterization is used. For this reason, an example-based approach that directly controls the facial geometry should make use of the coupled local models introduced by the WPSD scheme [120].

A hybrid approach. Blendshapes are based on simple linear interpolation, which leaves much to be desired, particularly when several shapes are combined. One solution involves adding a number of correction shapes [174, 212]. Each such shape is a correction to a pair (or triple, quadruple) of primary shapes. While this improves the interpolation, it is an expensive solution due to the number of possible combination shapes. Finding the correct shapes also involves a labor-intensive trial and error process. Typically a bad shape combination may be visible at some arbitrary set of 


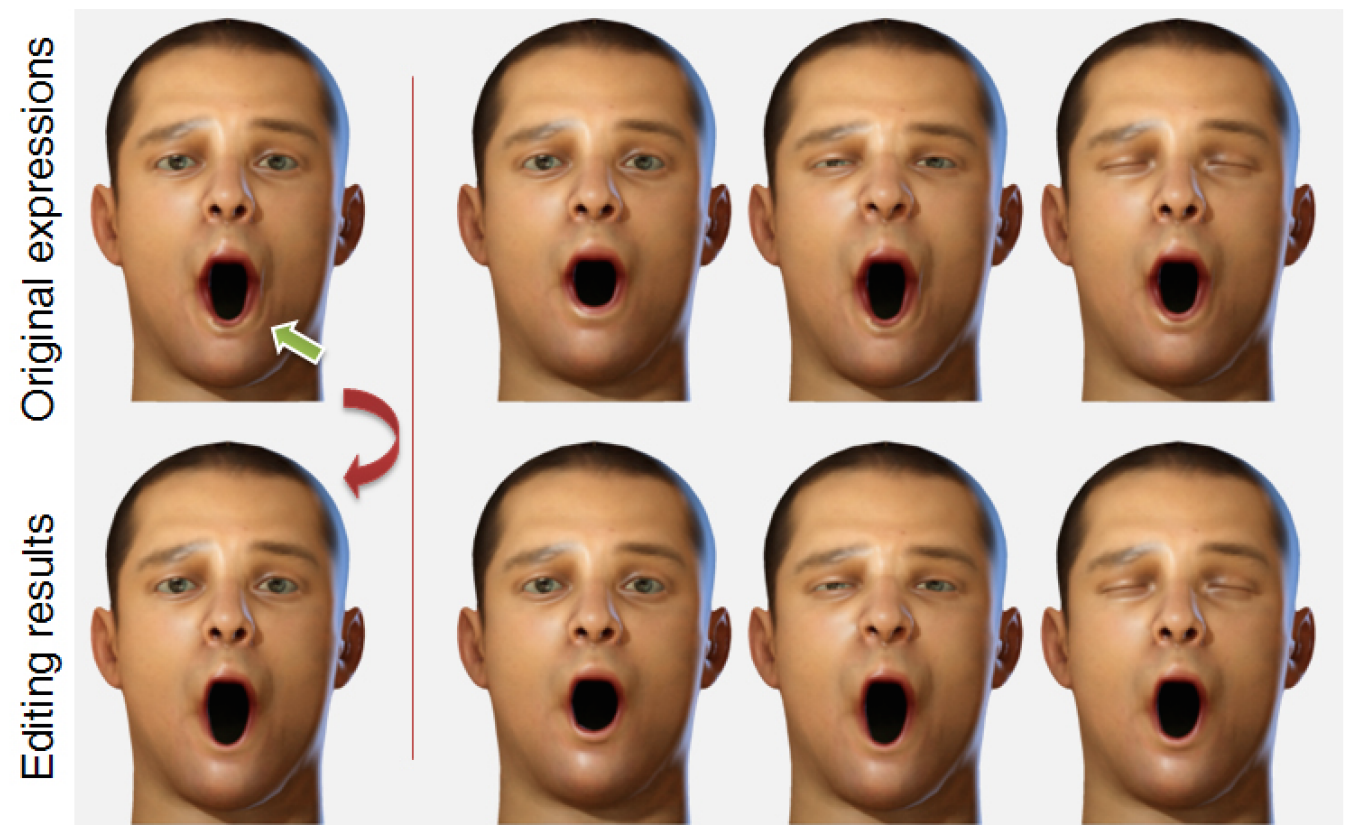

Figure 7.6: Smoothly propagating an expression change to nearby expressions using Weighted Pose Space Editing [216]. 
weights such as $\left(w_{10}=0.3, w_{47}=0.65\right)$. The blendshape correction scheme does not allow a correction to be associated with this location, however, so the artist must find corrections at locations (vertices of the weight hypercube) such as $\left(w_{10}=1, w_{47}=.1\right),\left(w_{10}=1, w_{47}=0\right),\left(w_{10}=0, w_{47}=1\right)$ that together add to produced the desired shape. This requires iterative resculpting of the relevant shapes.

To solve this problem, Seol et al. [216] combine ideas from blendshapes and weighted pose space deformation. Specifically, they use an elementary blendshape model (without corrections) as both a base model and a pose space for interpolation. Then, the weighted PSD idea is used to interpolate additional sculpted shapes. These shapes can be situated at any location in the weight space, and they smoothly appear and fade away as the location is approached (Figure 7.6). Because of the unconstrained location of these targets and the improved interpolation, both the number of shapes and the number of trial-and-error sculpting iterations are reduced.

\subsubsection{Wrinkles and Clothing}

[29] developed a multi-scale facial animation system in which the large scale motion interpolates the motion capture or user-defined controls. WPSD is used to drive medium-scale dynamic wrinkles. The "pose space" in this case is the relative stretch of edges on the skin mesh. Example-based methods have been employed to generate the deformation of clothing. [265] used WPSD to add dynamic wrinkle detail to a low-resolution cloth model.

\subsubsection{Retargeting}

[200] describes a system in which facial movement is tracked from a single camera and transferred to an avatar. The retargeting component of this system first deforms the avatar mesh to produce the basic facial expression. This step introduces geodesic radial basis function (GRBF) inter- 
polation: Rather than accomplishing the deformation using RBF regression evaluated over distances in Euclidean 3-D space as is done in [171], geodesic distances on the surface of the face are used. This has the advantage that the distance has to "go around" the mouth and eyes, so that moving the lower lip (for example) does not pull the upper lip along with it.

The GRBF approach can be considered to be dual to deformation using mesh Laplacian interpolation [28] and has some of the same advantages. The GRBF approach has some computational advantages, however. For example, the initial weight solve involves a linear system in the number of control points rather than in the number of interpolated vertices (see section 8.2.5.

Following this global mesh deformation, [200] uses PSD to apply individualized avatar-specific detail to the facial expressions. The vector of tracked control points forms the pose space in this case.

\subsection{Chapter Summary}

PSD is a prototypical "example based" algorithm in computer graphics. The idea is relatively well suited to the assumptions of the modern movie and games industries, since skilled artists can directly sculpt examples of the desired character shapes - a very intuitive task. The remaining work of producing deformation under movement of the character is recast as a relatively simple interpolation problem, albeit in a higher dimensional "pose space". The PSD algorithm has seen some industry adoption [127, 117, 46], and the general approach has inspired further academic research as described in this chapter.

This chapter has reinterpreted PSD and related algorithms from the machine-learning informed point of view adopted in this thesis. We observed that PSD can be considered as defining a "manifold" of character deformations upon which example shapes are manually situated, as op- 
posed to methods that interpolate in the geometric space defined by the model.

The next chapter continues our discussion of example-based skinning, covering the underlying scattered interpolation algorithms and some algorithmic and numerical issues. 


\section{Chapter 8}

\section{Scattered Interpolation Algorithms and Considerations}

Example-based scanning algorithms such as PSD have typically been posed in terms of an underlying scattered interpolation or approximation algorithm, most often using RBF. This chapter provides an implementer's guide to RBF, including the choice of kernel, and description of variants such as normalized RBF and Laplacian splines implemented as RBF. (The author's SIGGRAPH courses [137, 9] have more detail on scattered interpolation methods other than RBF.)

We also describe practical issues of handling bad data and unintentionally duplicated data. The chapter concludes with a novel intuitive explanation of the origins of the RBF kernel as a Green's function, using only linear algebra.

\subsection{Deformation as Scattered Interpolation}

In abstract, we wish to express the deformation of a surface as a function of either the pose of an underlying skeleton, or equivalently as a function of some other set of parameters such as the $\{$ smile, raise-eyebrow,... $\}$ controls desirable in facial animation. We also wish to directly sculpt the desired 


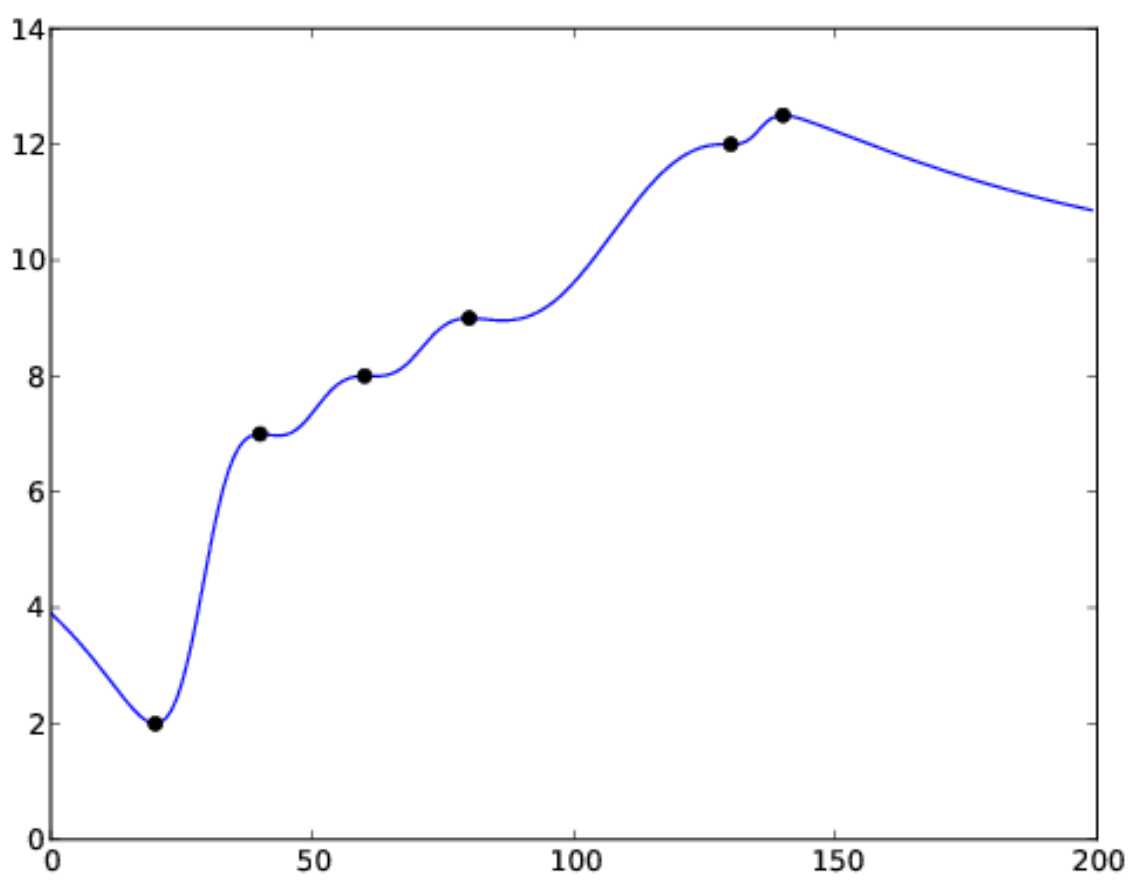

Figure 8.1: Shepard's interpolation with $p=2$. Note the the derivative of the function is zero at the data points, resulting in smooth but uneven interpolation. This same set of points will be tested with the RBF interpolation methods discussed below.

deformation at various points in the pose space, rather than working in a more abstract space such as the coefficients on various transforms as required by the LBS algorithm.

A scattered data interpolation method is required because deformations will be sculpted at arbitrary (rather than regularly spaced) poses. Since this interpolation is central to our application (the results of the interpolation will be directly visible as the animating deformation), we will consider the available scattered interpolation approaches.

In theory an example-based skinning algorithm such as PSD can be 


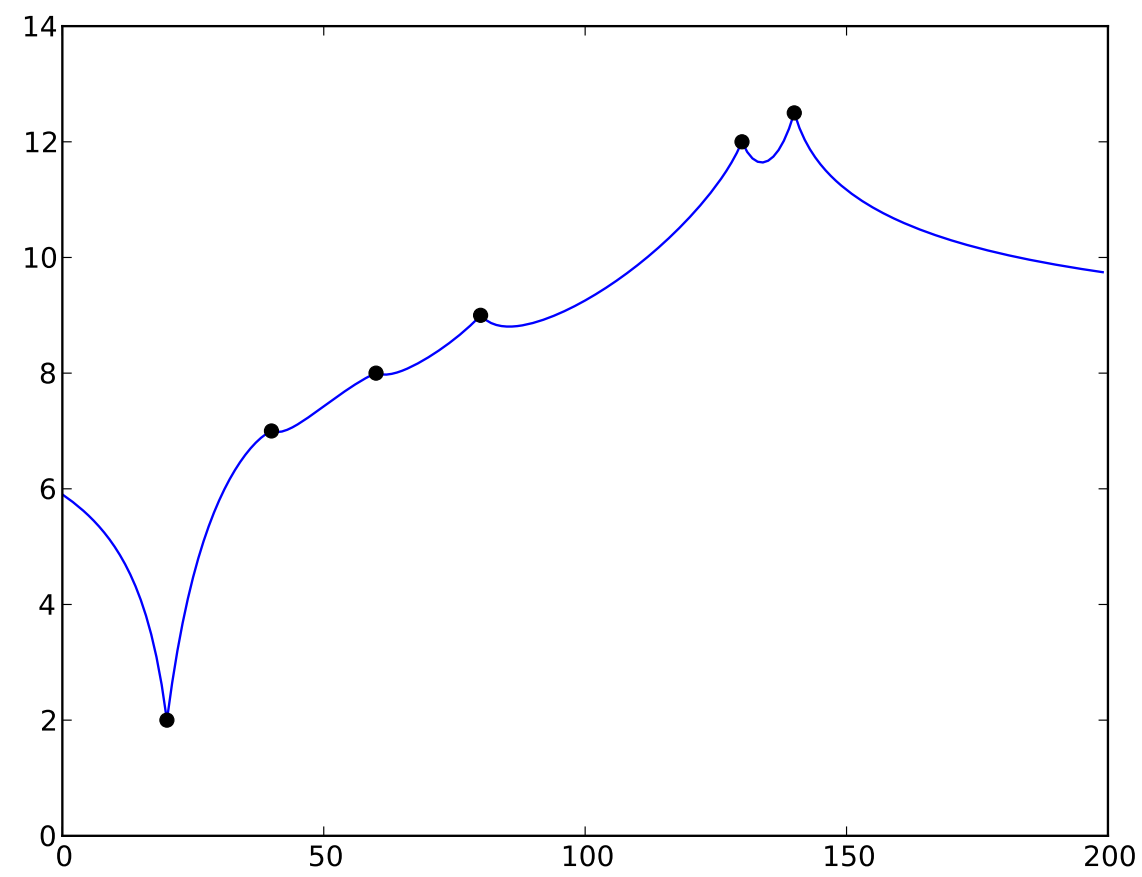

Figure 8.2: Shepard's interpolation with $p=1$.

implemented using any scattered interpolation scheme. For example the author is aware of one implementation based on natural neighbor interpolation [218].

\subsubsection{Linear Interpolation}

Interpolation generally involves a trade-off between smoothness and overshoot: linear interpolation is not smooth, but the function takes on its extreme values exactly at the datapoints; increasing smoothness typically causes the interpolated function to "overshoot" or travel beyond the range of the data. Because overshoot in high-dimensional functions is difficult to visualize and control, [23] introduced a variant of pose space deformation 
that uses simple linear interpolation on the data simplices. ([126] may have also used this idea, though it is difficult to say from the one-page description). This approach sacrifices smoothness but prevents overshoot; this was the recommendation in the evaluation [126].

Implementing linear interpolation requires first "triangulating" the domain of example poses into a set of simplices. A simplex is the $n$-dimensional generalizations of a triangle: a triangle is 3 points in two dimensions, a tetrahedron is 4 points in three dimensions, and a general simplex is $d+1$ points in $d$ dimensions. Each "point" is a location in the pose space, with an associated geometric pose of the creature. Computational geometry libraries such as qdelaunay can be used for this purpose [23]. After the simplices are obtained, interpolation can be performed using the $d$ dimensional generalization of barycentric interpolation. Specifically, the weights on the vertices of a simplex can be found by solving a block linear system

$$
\left[\begin{array}{l}
\mathbf{P} \\
\mathbf{1}
\end{array}\right][\mathbf{w}]=\left[\begin{array}{l}
\mathbf{p} \\
1
\end{array}\right]
$$

Here $\mathbf{P}$ is a $d \times d+1$ matrix whose columns contain the locations of the vertices of the simplex, so the upper equation $\mathbf{P w}=\mathrm{p}$ is expressing a particular location $p$ as a weighted sum of the vertices. 1 is a row vector of ones, so the bottom row $\mathbf{1}^{T} \mathbf{w}=1$ requires that the sum of the weights be one. If all the weights are non-negative the location $\mathrm{p}$ is inside the simplex. Then, the geometry at this location can be obtained as a weighted sum of the geometry associated with each vertex of the simplex, using $\mathrm{w}$ as the weights. If any of the weights are below zero, then the particular point $\mathbf{p}$ is outside of this simplex, and other simplices should be tested.

In addition to lack of smoothness, linear interpolation is impractical in high dimensions because the number of simplices adjacent to a particular vertex grows factorially with the dimension. 


\subsubsection{Splines with Tension}
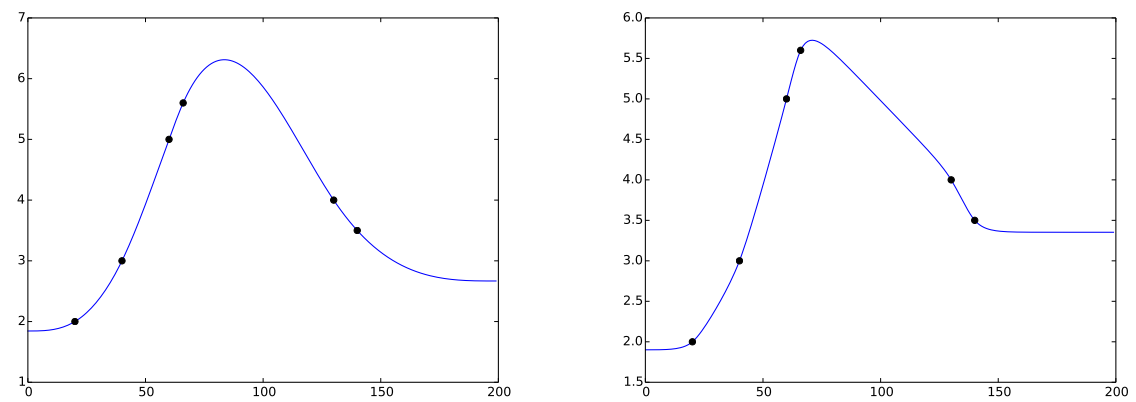

Figure 8.3: Spline with tension. Left, cubic spline interpolation. Right, cubic spline interpolation of the same points, with tension. The boundary conditions are set to have zero slope (Dirichlet).

The tradeoff between smoothness and overshoot is a fundamental issue in interpolation. Using linear interpolation is not the only way to control overshoots. Splines with "tension" result from minimizing an objective that includes a weighted sum of the squared first and higher order derivatives, integrated over the function (Equation (8.2)). The weights provide a "knob" that allows the amount of smoothness to be controlled. Splines with tension can in theory be implemented through radial basis interpolation (section 8.1.4), although the RBF kernel function for this purpose has not been worked out in higher dimensions. Shepard's method (Section 8.1.3 can produce an interpolated function whose derivative is zero at the gradients. This may be considered as an artifact, or an advantage, since it has the effect of reducing overshoot.

\subsubsection{Shepard's Method}

Shepard's method [16, 21] is a frequently employed scattered data interpolation scheme in computer graphics. In this method the interpolated value 
is a weighted sum of the surrounding data points normalized by the sum of the weights,

$$
\hat{d}(\mathbf{p})=\frac{\sum w_{k}(\mathbf{p}) d_{k}}{\sum w_{k}(\mathbf{p})}
$$

with weights set to an inverse power of the distance: $w_{k}(\mathbf{p})=\left\|\mathbf{p}-\mathbf{p}_{k}\right\|^{-p}$. (This is singular at the data points $\mathbf{p}_{k}$ and should computed as $\left(\left\|\mathbf{p}-\mathbf{p}_{k}\right\|+\right.$ $\epsilon)^{-p}$ ). With $p>1$ the interpolated function is once differentiable. Unfortunately this simple scheme has some potentially undesirable properties. Far from the data the weights will be approximately the same, $\hat{d}(\infty)=w_{\infty} \sum d_{k} / w_{\infty} \sum 1=\sum d_{k} / N$, i.e. the interpolated surface converges to the average of the data values. A serious drawback for some applications is that the derivative of the surface is zero at the data points (Figure 4).

This problem can be addressed by using moving least squares schemes [58, 121]. These involve fitting a low-order polynomial over a sliding window of nearby points, using a weighted least squares scheme to reduce the influence of points at the edge of the neighborhood. While moving least squares could be used to implement pose space deformation, the training example poses may have very uneven distribution in the pose space, thus making the notion of "neighborhood" fragile. Radial basis functions (described below) are an alternate choice.

\subsubsection{Radial Basis Functions}

Radial basis functions [194, 195] have become a popular choice for scattered interpolation. The interpolant is a linear combination of nonlinear functions of distance from the data points (Figure 8.4):

$$
\hat{d}(\mathbf{p})=\sum_{k}^{N} w_{k} R\left(\left\|\mathbf{p}-\mathbf{p}_{k}\right\|\right)
$$




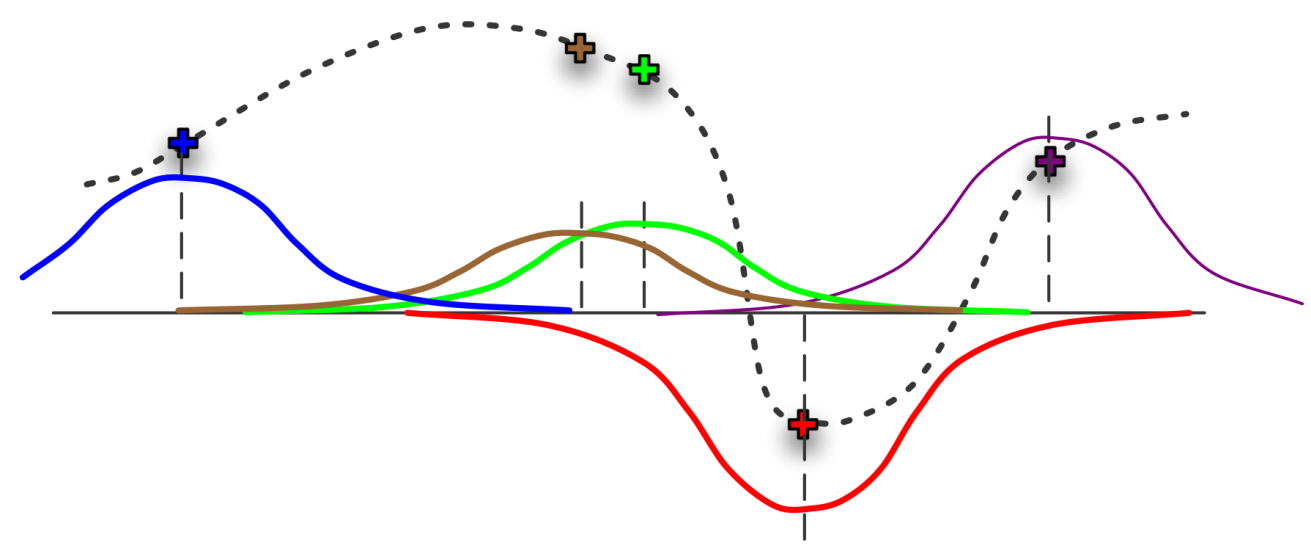

Figure 8.4: Schematic illustration of RBF interpolation in one dimension. A fixed-width kernel is situated at each data point (solid curves). The weighted sum of these interpolates the given data (dashed line). The weights are obtained by solving a linear system.

If $N$ values of $d$ are available then the weights can be easily solved by a linear system of the form

$$
\left[\begin{array}{cccc}
R_{1,1} & R_{1,2} & R_{1,3} & \cdots \\
R_{2,1} & R_{2,2} & \cdots & \\
R_{3,1} & \cdots & & \\
\vdots & & &
\end{array}\right]\left[\begin{array}{c}
w_{1} \\
w_{2} \\
w_{3} \\
\vdots
\end{array}\right]=\left[\begin{array}{c}
d_{1} \\
d_{2} \\
d_{3} \\
\vdots
\end{array}\right]
$$


(compactly, $\mathbf{R w}=\mathbf{d}$ ) where $R_{i, j}$ denotes $R\left(\left\|\mathbf{p}_{i}-\mathbf{p}_{k}\right\|\right)$ and $d_{i}$ are the data points to interpolate, giving the solution!

$$
\mathbf{w}=\mathbf{R}^{-1} \mathbf{d}
$$

The distance ||| can be generalized to Mahalanobis distance (effectively rotating and stretching the basis function) [32].

\section{Normalized RBF interpolation}

Radial basis functions with polynomial terms are most often used for kernels that generate "Laplacian splines" (section 8.2.2). An attractive feature of these splines is that the kernel has no parameters to adjust.

Some commonly used RBF kernels, such as the Gaussian, have a width parameter that must be set. If the width is too small, the reconstruction function decays to zero between the data, whereas a larger width causes the function to overshoot (Figures 8.5 8.7). An overly large width can sometimes cause wild oscillations.

\footnotetext{
${ }^{1}$ The paper [139] described a geometric derivation, in which the available data points are considered as a single point $\mathbf{d}$ in an $N$ dimensional space. The best approximation to $\mathbf{d}$ in the space spanned by $\mathbf{R}$ occurs (in direct analogy with the three-dimensional case) when the weights are such that the error $\mathbf{d}-\mathbf{R w}$ is orthogonal to each column of $\mathbf{R}$ :

$$
\mathbf{R}^{T}(\mathbf{R} \mathbf{w}-\mathbf{d})=\mathbf{0}
$$

giving the normal equation form

$$
\mathbf{R}^{T} \mathbf{R} \mathbf{w}=\mathbf{R}^{T} \mathbf{d}
$$

that can be solved for

$$
\mathbf{w}=\left(\mathbf{R}^{T} \mathbf{R}\right)^{-1} \mathbf{R}^{T} \mathbf{d}
$$

The normal equations form generalizes to the case where there are fewer RBF kernels than data points, as arises with some model simplification schemes. However in the simple case where an RBF kernel is centered at each data point, the normal equations form provides no advantage and on the contrary has a poorer condition number due to the squaring of the $\mathbf{R}$ matrix.
} 


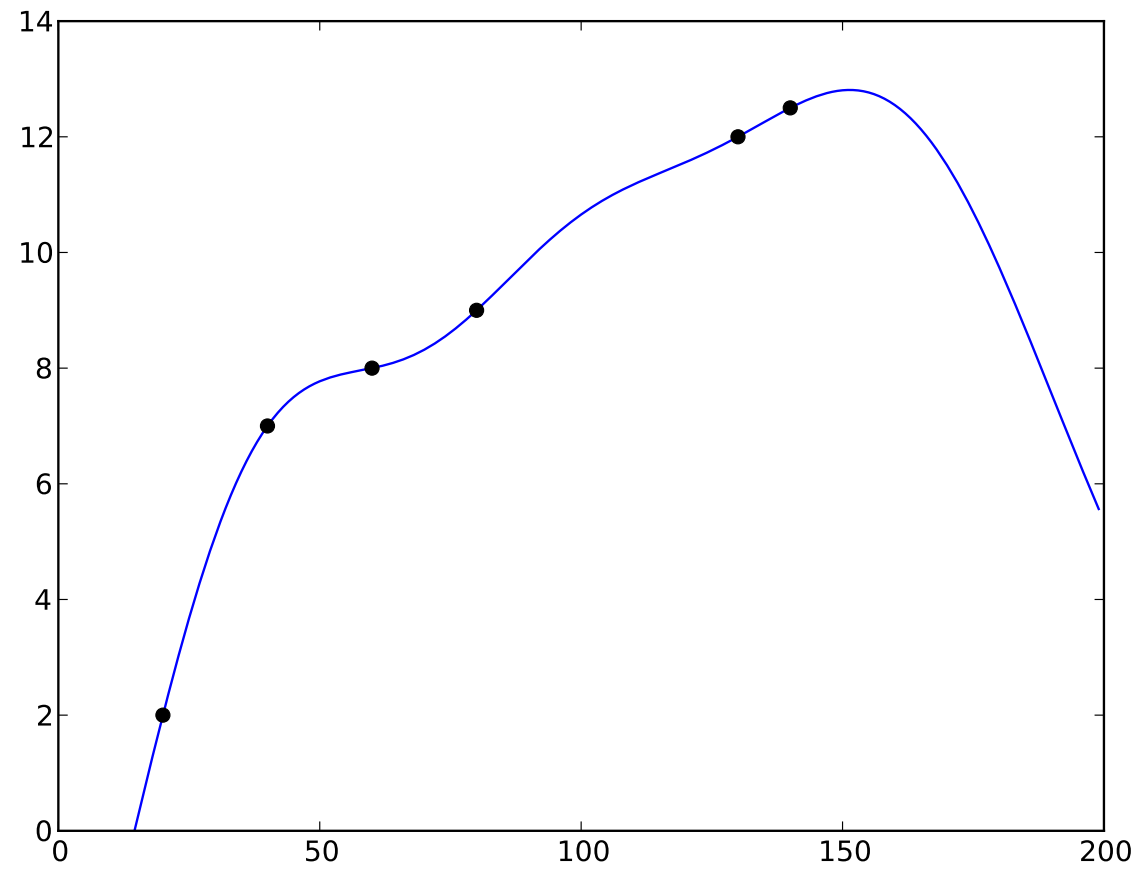

Figure 8.5: One-dimensional radial basis interpolation with a Gaussian kernel. The curve extrapolates to zero.

The width parameter can be set by cross validation, or conceivably by invoking the RBF-Gaussian process equivalence [8] and optimizing the (log) marginal likelihood with respect to the hyperparameters [199, (2.30)]. Unfortunately, if the data has different densities in different areas, no single parameter choice will be suitable everywhere.

The normalized RBF scheme [164] produces interpolation that is less sensitive to the choice of the width parameter. In this scheme, the basis functions are normalized to sum to one,

$$
\hat{d}(\mathbf{p})=\frac{\sum_{k}^{N} w_{k} R\left(\left\|\mathbf{p}-\mathbf{p}_{k}\right\|\right)}{\sum_{k}^{N} R\left(\left\|\mathbf{p}-\mathbf{p}_{k}\right\|\right)}
$$

Even with a poorly chosen kernel width (Figure 8.8) the interpolation does 


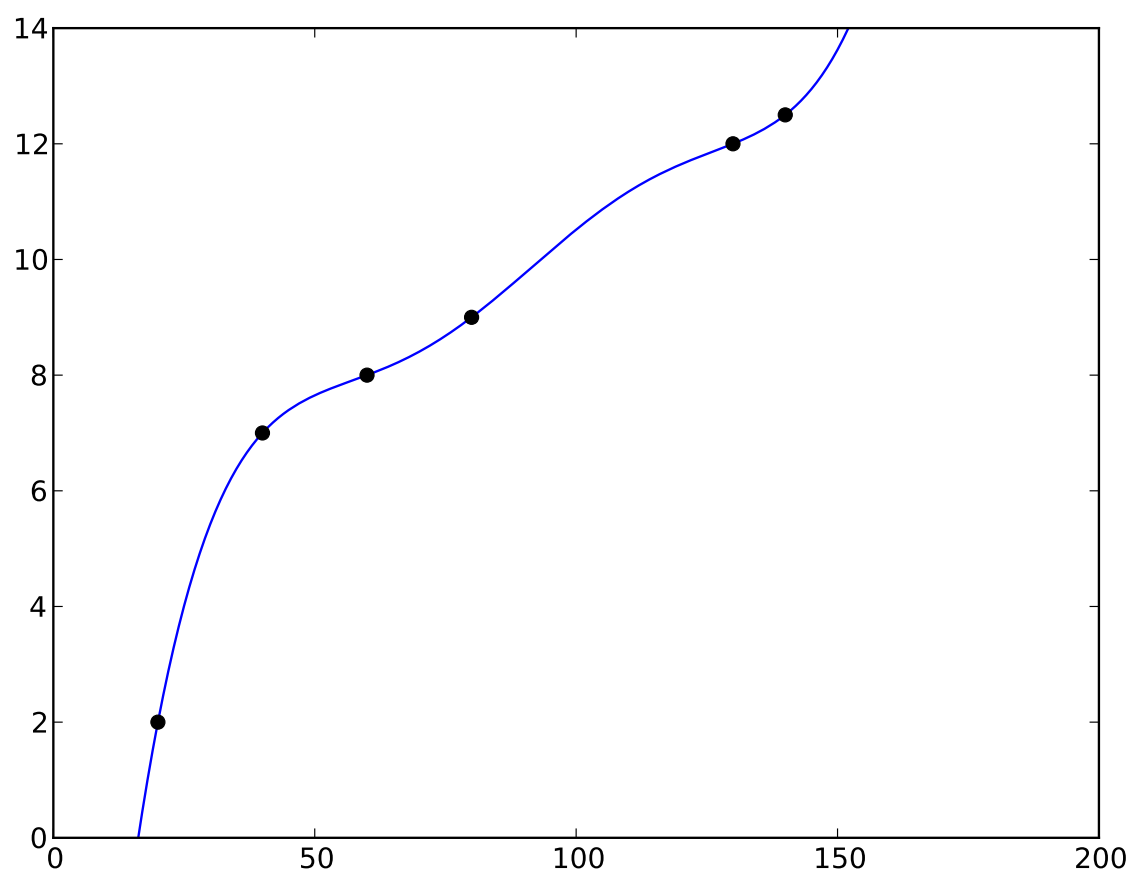

Figure 8.6: Radial basis interpolation with a Gaussian kernel, varying the kernel width relative to Fig. 8.5

not overshoot significantly or drop to zero between the data points.

\section{What is the right RBF kernel?}

Different choices of the RBF kernel function $R$ are possible. Kernels corresponding to $n$-harmonic splines are discussed in Section 8.2. The choice involves the definition of smoothness and the tradeoff between smoothness and overshoot.

The Gaussian kernel involves a width parameter $\sigma$, as do some other kernels. It is possible to choose this width parameter to locally adapt to the data, so that kernels centered at different locations have different widths [32], however the resulting problem is not convex, and a more expensive 


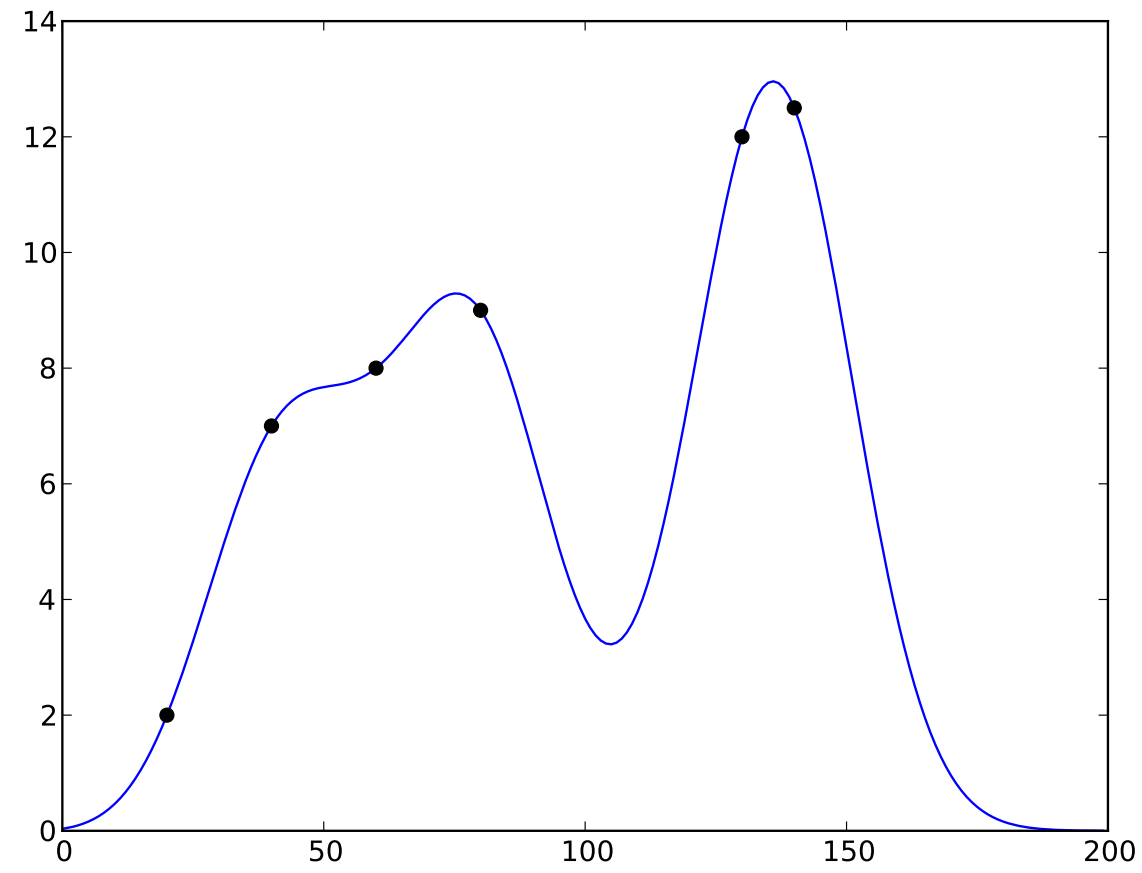

Figure 8.7: Radial basis interpolation with a Gaussian kernel, varying the kernel width relative to Fig. 8.5[8.6. In this figure the kernel width is too narrow to adequately interpolate the data.

nonlinear optimization would be required.

A practical recommendation is to use normalized radial basis functions in combination with cardinal interpolation, as is done in WPSD. Normalized radial basis functions are somewhat less sensitive to the choice of kernel width. Cardinal interpolation has a data-adaptive effect by forcing the interpolated function to zero at the locations of other training examples. 


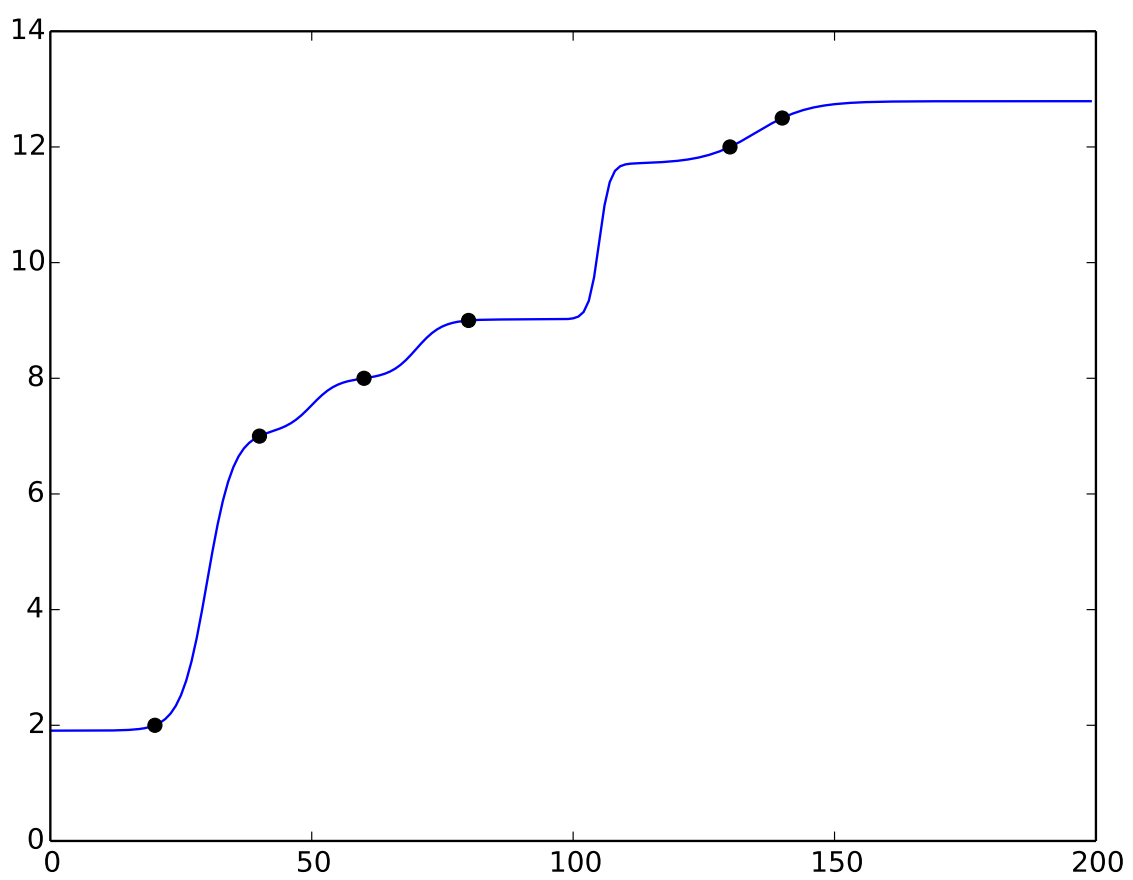

Figure 8.8: Normalized RBF interpolation using a Gaussian kernel. The normalized RBF scheme produces interpolation that is less sensitive to poor choices of the width parameter. 


\subsubsection{Interpolation of 3D Data}

The preceding description maps a $k$-dimensional input space (arbitrary $k$ ) to a one dimensional range, i.e., it is the $k$-dimensional version of a height field. Three-dimensional data can be interpolated by using three separate one-dimensional interpolations, one each for $x, y, z$. In the case of radial basis function interpolation, the $\mathbf{R}$ matrix is common to the three dimensions and can be inverted just once.

\subsection{Numerical Considerations}

A variety of functions can be used as the radial basis kernel $R(r)$. Some of the most common choices in the literature are (c.f. [85, Appendix D]):

\section{Gaussian}

Hardy multiquadric

Inverse multiquadric

Thin plate spline

$$
\begin{aligned}
& R(r)=\exp \left(-(r / c)^{2}\right) \\
& R(r)=\sqrt{r^{2}+c^{2}} \\
& R(r)=1 / \sqrt{r^{2}+c^{2}} \\
& R(r)=r^{2} \log r \quad \text { (in two dimensions) }
\end{aligned}
$$

Laplacian (or Polyharmonic) splines $R(r) \propto \begin{cases}r^{2 s-n} \log r & 2 s-n \text { even } \\ r^{2 s-n} & \text { otherwise }\end{cases}$

In fact there is not yet a general characterization of what functions are suitable as RBF kernels [85]. Positive definite functions are among those that will generate a non-singular $\mathbf{R}$ matrix for any choice of data locations. There are several alternate characterizations of positive definiteness:

- A function is positive definite if

$$
\sum_{i} \sum_{k} w_{i} w_{k} R\left(\left\|\mathbf{x}_{i}-\mathbf{x}_{j}\right\|\right)>0
$$

for any choice of unique data locations $\mathbf{x}_{i}$.

- A positive definite function is a Fourier transform of a non-negative function [138]. (Note that "positive definite" does not mean that the 


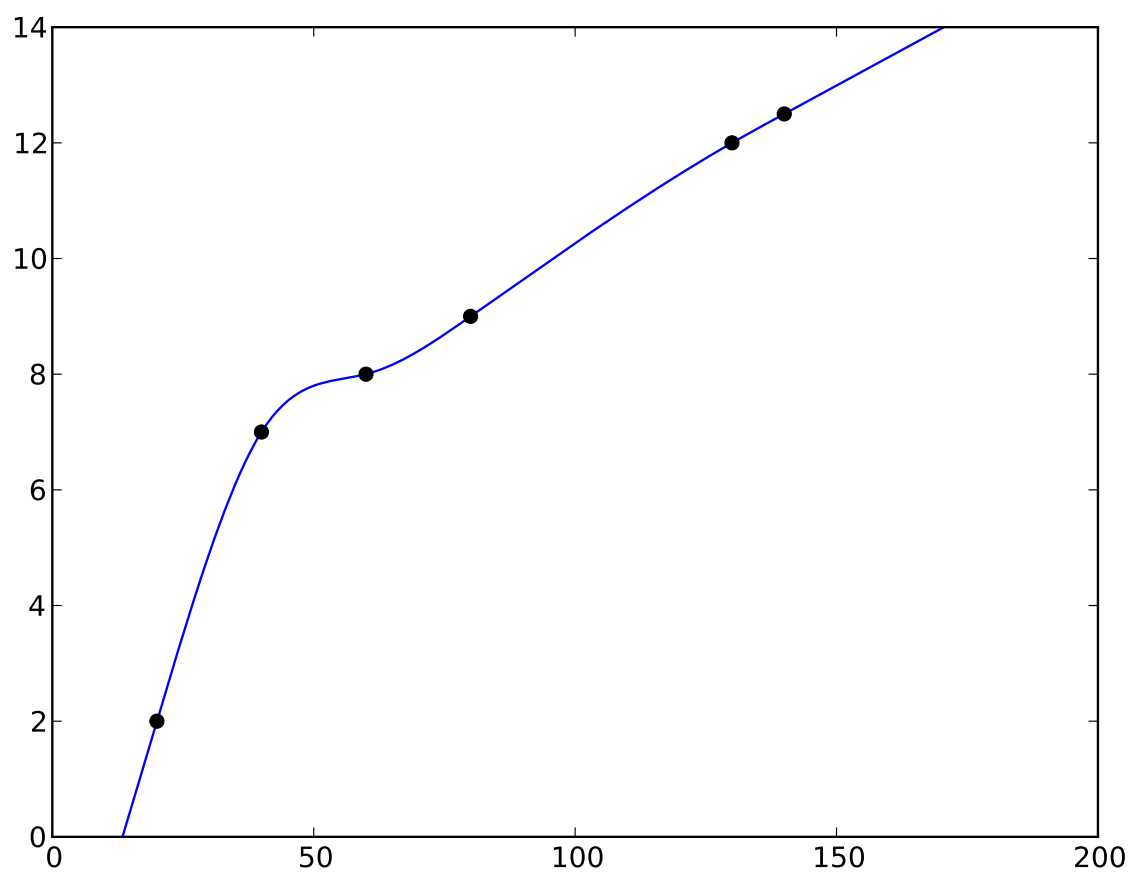

Figure 8.9: The one-dimensional equivalent of thin-plate spline interpolation is the natural cubic spline, with radial kernel $|r|^{3}$ in one dimension. This spline minimizes the integrated square of the second derivative (an approximate curvature) and so extrapolates to infinity away from the data. 


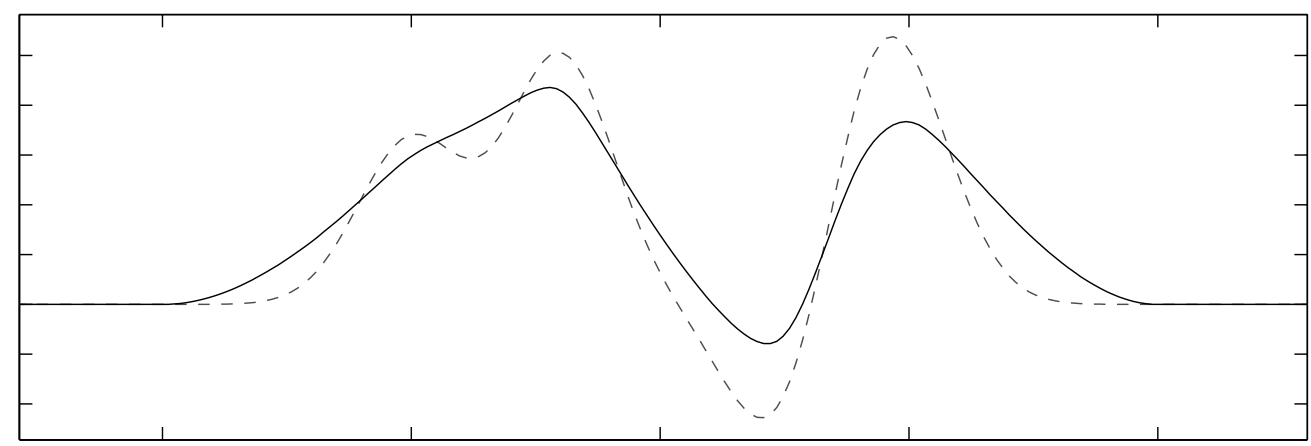

Figure 8.10: Comparison of the 3D biharmonic clamped plate spline (solid line) with Gaussian RBF interpolation (dashed line) [201].

function itself is necessarily positive. The sinc function is a counter example, since it is the transform of a box function.)

- The matrix $\mathbf{R}$ generated from a positive-definite kernel function has all eigenvalues positive.

The third characterization indicates that for a positive definite function, the matrix $\mathbf{R}$ will be invertable for any choice of data points. On the other hand, there are useful kernels (such as the polyharmonic family) that are not positive definite.

Several aspects of radial basis kernels are not intuitive at first exposure. The polyharmonic and thin-plate kernels are zero at the data points and increase in magnitude away from the origin. Also, kernels that are not themselves smooth can combine to give smooth interpolation. An example is the second-order polyharmonic spline in 3 dimensions, with kernel $R(r)=|r|$.

Several of the common RBF kernels have an explicit width parameter (e.g. the Gaussian $\sigma$ ). The use of a broad kernel can cause surprising effects. This is due to the near-singularity of the $\mathbf{R}$ matrix resulting from rows that are similar. The problem (and a solution) is discussed in section 
8.2.1.

When thinking about the appropriate kernel choice for an application, it is also worth keeping in mind that there is more than one concept of smoothness. One criterion is that a function has a number of continuous derivatives. By this criterion the Gaussian function is a very smooth function, having an infinite number of derivatives. A second criterion is that the total curvature should be small. This can be approximated in practice by requiring that the solution should have a small integral of squared second derivatives - the criterion used in the thin-plate and biharmonic splines.

This second criterion may correspond better to our visual sense of smoothness, see Figures 8.9, 8.10, Figure 8.9 shows a natural cubic spline on scattered data points. The curve extrapolates beyond the data, rather than falling back to zero, which may be an advantage or disadvantage depending on the application. Figure 8.10 compares two approaches that both fall to zero: Gaussian RBF interpolation (red) and the 3D biharmonic clamped plate spline (black line). The plot is the density of a linear section through an interpolated volume. Note that the control points are distributed through the volume and cannot meaningfully be visualized on this plot. Although the Gaussian function has an infinite number of derivatives, Gaussian RBF interpolation is visually less smooth than the biharmonic spline. The visual smoothness of the Gaussian can be increased by using a broader kernel, but this also increases the overshoots.

For many applications the most important considerations will be the smoothness of the interpolated function, whether it overshoots, and whether the function decays to zero away from the data. For example, artists tend to pick examples at the extrema of the desired function, so overshoot is not desirable. As another example, in the case where a pose space deformation algorithm is layered on top of an underlying skinning algorithm, it is important that the interpolated function fall back to zero where there is no data. 
Of course it is not possible to simultaneously obtain smoothness and lack of overshoot, nor smoothness and decay to zero away from the data. It is possible, however, to combine these criteria with desired strengths. The approach is to find the Green's function (see section 8.2.6) for an objective such as

$$
\begin{array}{r}
F(f)=\sum\left(f\left(x_{i}\right)-y_{i}\right)^{2}+\alpha \int\left\|\nabla^{2} f\right\|^{2} d x \\
+\beta \int\|\nabla f\|^{2} d x \\
+\gamma \int\|f\|^{2} d x
\end{array}
$$

i.e. a weighted sum of the data fidelity and the integral of the squared second derivative, the squared first derivative (i.e. "spline with tension"), and the squared function itself (causing the function to decay to zero).

The kernel choice leads to numerical considerations. The Gaussian kernel can be approximated with a function that falls exactly to zero resulting in a sparser and numerically better conditioned matrix [248], while the polyharmonic kernels are both dense and ill-conditioned.

\subsubsection{Regularization}

In creating training poses for example-based skinning, the artist may accidentally save several sculpted shapes at the same pose. This results in a singular $\mathbf{R}$ matrix. This situation can be detected and resolved by searching for identical poses in the training data.

A more difficult case arises when there are several (hopefully similar) shapes at placed at nearly but not exactly the same location in the pose space. (The case in which the shapes are not similar is treated in the next subsection). In this case the $\mathbf{R}$ matrix is not singular, but is poorly conditioned. The RBF result will probably pass through the given datapoints, but it may do wild things elsewhere (Figure 8.13). Intuitively speaking, the matrix is dividing by "nearly zero" in some directions, resulting in large overshoots. 


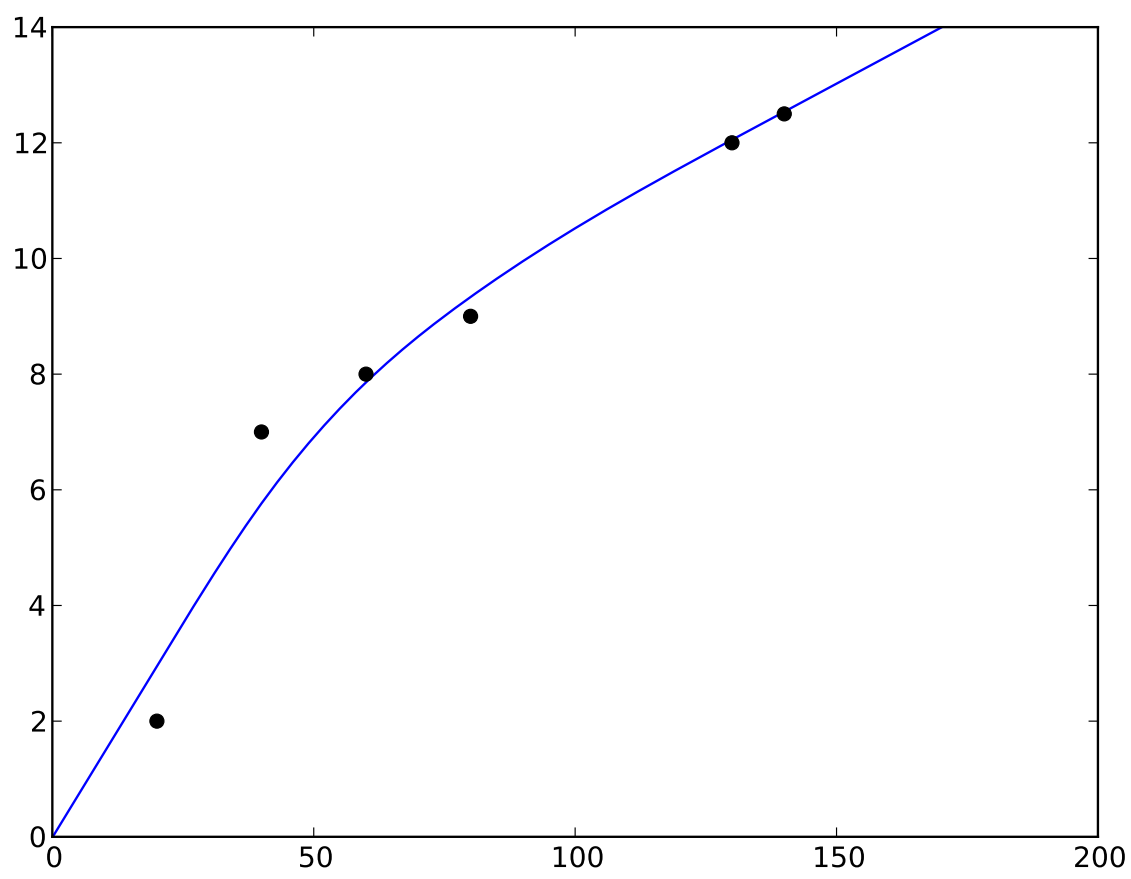

Figure 8.11: Adding regularization to the plot in Fig. 8.9 causes the curve to approximate rather than interpolate the data.

In this case an easy fix is to apply weight-decay regularization. Rather than requiring $\mathrm{Rw}=\mathrm{d}$ exactly, weight-decay regularization solves

$$
\arg \min _{\mathbf{w}}\|\mathbf{R w}-\mathbf{d}\|^{2}+\lambda\|\mathbf{w}\|^{2}
$$

This adds a second term that tries to keep the sum-square of the weight vector small. However, $\lambda$ is chosen as a very small adjustable number such as 0.00001 . In "directions" where the fit is unambiguous, this small number will have little effect. In directions where the result is nearly ambiguous however, the $\lambda\|\mathbf{w}\|^{2}$ will choose a solution where the particular weights are small.

To visualise this, consider a two-dimensional case where one basis vec- 

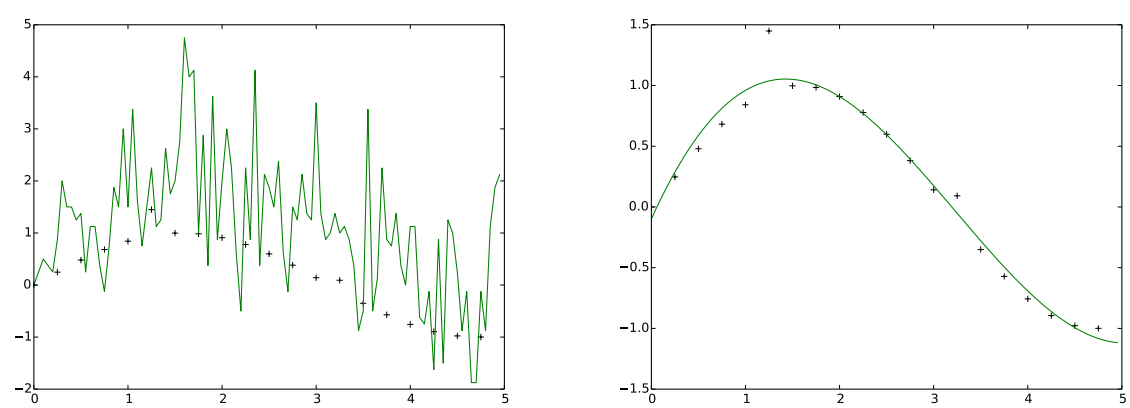

Figure 8.12: One-dimensional values interpolated with a Gaussian kernel that is too broad for the data spacing, resulting in bad conditioning. Left, no regularization. Right, regularization parameter 0.00001

tor is pointing nearly opposite to the other (say, at 179 degrees away). A particular point that is one unit along the first vector can be described almost as accurately as being two units along that vector, less one unit along the (nearly) opposite vector. Considering matrix inversion in terms of the eigen decomposition $\mathbf{R}=\mathbf{U} \boldsymbol{\Lambda} \mathbf{U}^{T}$ of the matrix is also helpful. The linear system is then $\mathbf{U} \mathbf{\Lambda} \mathbf{U}^{T} \mathbf{w}=\mathbf{d}$, and the solution is

$$
\mathbf{w}=\mathbf{U} \boldsymbol{\Lambda}^{-1} \mathbf{U}^{T} \mathbf{d}
$$

Geometrically, this is analogous to rotating $d$ by $\mathbf{U}^{-1}$, stretching by the inverse of the eigenvalues, and rotating back. The problematic directions are those corresponding to small eigenvalues.

Solving (8.3) for $\mathrm{w}$,

$$
\begin{aligned}
& \|\mathbf{R} \mathbf{w}-\mathbf{d}\|^{2}+\lambda\|\mathbf{w}\|^{2} \\
& =\operatorname{tr}(\mathbf{R} \mathbf{w}-d)^{T}(\mathbf{R} \mathbf{w}-\mathbf{d})+\lambda \mathbf{w}^{T} \mathbf{w} \\
& \frac{d}{d \mathbf{w}}=0=2 \mathbf{R}^{T}(\mathbf{R} \mathbf{w}-\mathbf{d})+2 \lambda \mathbf{w} \\
& \mathbf{R}^{T} \mathbf{R} \mathbf{w}+\lambda \mathbf{w}=\mathbf{R}^{T} \mathbf{d}
\end{aligned}
$$



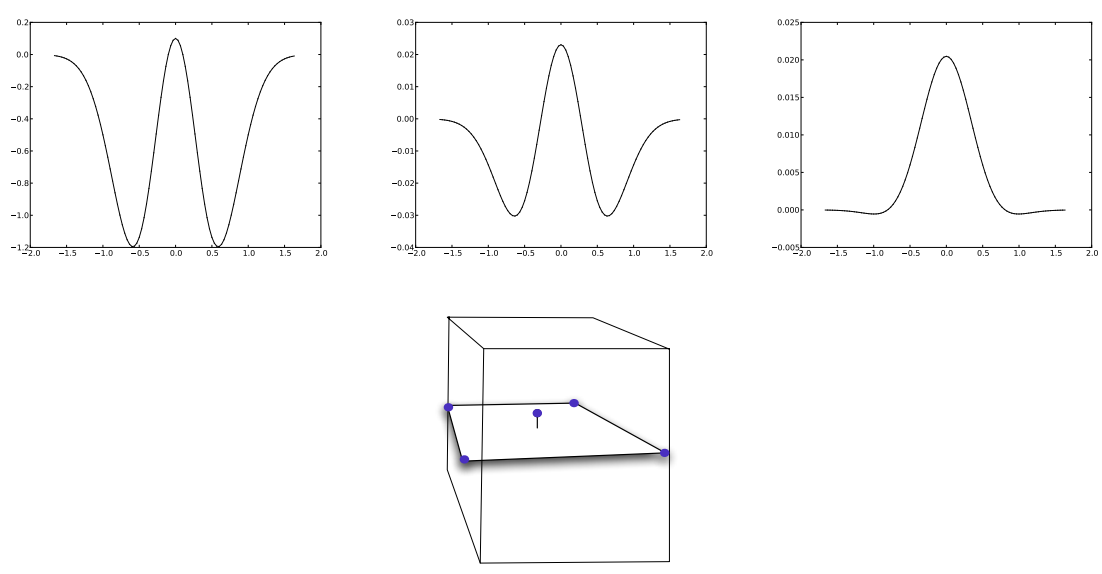

Figure 8.13: Illustration of ill-conditioning and regularization in a 3D example. From left to right, the regularization parameter is $0, .01$, and .1 respectively. Note the vertical scale on the plots is changing. Bottom: The geometry of this 3D interpolation problem. The data are zero at the corners of a square, with a single non-zero value in the center. The plots show a single section through the interoplated function.

giving the solution

$$
\mathbf{w}=\left(\mathbf{R}^{T} \mathbf{R}+\lambda \mathbf{I}\right)^{-1} \mathbf{R}^{T} \mathbf{d}
$$

In other words, weight decay regularization requires just adding a small constant to the diagonal of the (squared) matrix $\mathbf{R}^{T} \mathbf{R}$ before inverting.

Fig. 8.13 shows a section through simple a two-dimensional interpolation problem using a Gaussian RBF kernel. The data are zero at the corners of a square, with a single non-zero value in the center. Noting the vertical scale on the plots, the subfigure on the left show wild oscillation that is not justified by the simple data. In the other subfigures we used weightdecay regularization to achieve a more sensible solution. This also has the effect of causing the reconstructed curve to approximate rather than interpolate the data (Fig. 8.11). When used with an RBF kernel that has an interpretation as a Green's function, weight decay regularization has been called shrinkage since it "shrinks" the interpolated function towards the 
null space of the corresponding differential operator.

Weight-decay regularization is not the only type of regularization. Regularization is an important and well studied topic in all areas involving fitting a model to data. On a deeper level it is related to model complexity and the bias-variance tradeoff in model fitting. The subject comes up in a number of forms in statistical learning texts such as [100].

\subsubsection{Laplacian Spline and Thin-Plate RBF models}

The RBF kernels corresponding to Laplacian splines are augmented with a low order polynomial,

$$
f(x)=\sum_{k}^{n} c_{k} R\left(\left\|x-x_{k}\right\|\right)+\sum d_{j} p_{j}(x)
$$

with $p_{1}(x)=1$ is a constant term. This subsection motivates this alternate formulation.

The RBF and polynomial weights $\mathbf{c}, \mathbf{d}$ can be solved with a block matrix system

$$
\left[\begin{array}{ll}
\mathbf{R} & \mathbf{P} \\
\mathbf{P}^{T} & \mathbf{0}
\end{array}\right]\left[\begin{array}{l}
\mathbf{c} \\
\mathbf{d}
\end{array}\right]=\left[\begin{array}{l}
\mathbf{f} \\
\mathbf{0}
\end{array}\right]
$$

where $\mathbf{P}$ is the polynomial basis evaluated at all the data points. For example in the 2D thin-plate case a 2D linear polynomial is used, so $\mathbf{P}$ is a $3 \times n$ matrix containing in its columns the constant function, the $x$ location of each control point, and the $y$ location of each control point. Note that this matrix is indefinite (has both positive and negative eigenvalues), which rules out the use of Choleski and conjugate gradient solvers.

Intuitively, the need for a polynomial term arises because these polynomials are in the null space of the differential operator. For example, the thin-plate spline minimizes the squared second derivative of the unknown function, integrated over the function. A constant or linear term can be added to the function without affecting this functional. Thus, the thin-plate criterion specifies the interpolation up to a linear polynomial, 
which must also be estimated. As another example, the criterion of minimizing the integrated squared gradient is invariant to a constant offset, so the additional polynomial is simply this constant. ${ }^{2}$ However the augmented system Equation (8.4) can also be used with kernels such as the Gaussian.

The additional polynomial coefficients have to be determined somehow. Since the degrees of freedom in the $n$ points are already used in estimating the RBF coefficients $c_{k}$, an additional relation must be found. This takes the form of requiring that the interpolation should exactly reproduce polynomials: if the data $f(x)$ to be interpolated is exactly a polynomial, the polynomial contribution $c_{n+1} \cdot 1+c_{n+2} \cdot x+c_{n+3} \cdot y$ should be exactly that polynomial, and the weights on the RBF kernel $c_{k}, k \leq n$ should be zero.

Doing so results in the additional condition that the weights $c_{k}$ are in the null space of the polynomial basis. In showing this we use the following notation: $\mathbf{R}$ is the standard RBF system matrix with the RBF kernel evaluated at the distance between pairs of training points, $\mathrm{c}$ are the RBF weights (coefficients), and $\mathbf{f}$ are the desired values to interpolate, so $\mathbf{R c}=\mathbf{f}$ would be the system to solve if the extra polynomial is not used. Also let $\mathrm{P}$ be the polynomial basis, and $\mathbf{d}$ be the weights on the polynomial basis. In the 2D case with a linear polynomial $\mathbf{P}$ is a $n$ by 3 matrix with a column of ones, a column of $x_{k}$, and a column of $y_{k}$, where $\left(x_{k}, y_{k}\right)=\mathbf{p}_{k}$ are the training locations. Then,

\footnotetext{
${ }^{2}$ This is related to the fact that the constant function is in the null space of a Laplacian matrix.
} 
$\mathbf{R c}+\mathbf{P d}=\mathbf{f} \quad$ the interpolation statement $\mathbf{R c}+\mathbf{P d}=\mathbf{P m} \quad$ fit a polynomial $\mathbf{P m}$, for some unknown coeffs. $\mathbf{m}$ $\mathbf{c}^{T} \mathbf{R} \mathbf{c}+\mathbf{c}^{T} \mathbf{P d}=\mathbf{c}^{T} \mathbf{P m} \quad$ premultiply by $\mathbf{c}^{T}$ $\mathbf{c}^{T} \mathbf{R} \mathbf{c}=\mathbf{c}^{T} \mathbf{P m}-\mathbf{c}^{T} \mathbf{P d}$ $\mathbf{c}^{T} \mathbf{P}(\mathbf{m}-\mathbf{d})=\mathbf{c}^{T} \mathbf{R} \mathbf{c}$

Then if we require that $\mathbf{c}^{T} \mathbf{P}=\mathbf{0}$, then the left hand side is zero, and so $\mathbf{c}$ must itself be zero since the right hand side is positive. So now going back to the original $\mathbf{R c}+\mathbf{P d}=\mathbf{P m}$, we know that if $\mathbf{c}^{T} \mathbf{P}=\mathbf{0}$, then $\mathbf{c}=\mathbf{0}$, so $\mathbf{R c}=\mathbf{0}$, so Pd $=$ Pm.

Restating, this means that if the signal is polynomial and we have required $\mathbf{c}^{T} \mathbf{P}=\mathbf{0}$, the coefficients $\mathbf{c}$ are zero, and then $\mathbf{P d}=\mathbf{P m}$, so $\mathbf{d}=\mathbf{m}$, so the polynomial is exactly reproduced.

Another way of viewing these side conditions is that by imposing an additional relation on $\mathbf{c}$, they reduce the total number of degrees of freedom from $n+p$ (for a $p$-order polynomial basis) back to $n$

The additional relation $\mathbf{c}^{T} \mathbf{P}=\mathbf{0}$ is enough to solve for the RBF and polynomial coefficients using Equation 8.4 .

Reproducing a polynomial is also a useful thing in some applications. Thin-plate splines are commonly used for image registration (e.g. [133]), and in that application it would be troubling if an affine transformation could not be produced. Also, by regularizing the upper rows of the system matrix, the image warp is forced to become more affine [77].

\subsubsection{Detecting Bad Data}

Signal models often include a treatment of noise in the data. In this section we argue that the types of errors we find in data produced by artists is of a different character than that usually considered. We explore an approach 
for dealing with this type of errors that is also naturally formulated in a nonparametric model.

The standard assumption in signal modeling is that all data points are corrupted with some noise, for example with a Gaussian distribution of some assumed variance. In computer graphics models such as pose space deformation and blendshapes, we generally assume that the artist sculpts exactly the geometry that they desire. These shapes are to be exactly interpolated - there is no notion of "noise".

On the other hand, artists do make errors. Thus, a better characterization of our problem is that the artist usually produces perfect data, but in rare cases produces mistakes. A concrete example of this is in producing training data for the pose space deformation algorithm. The artist will sculpt exactly the shapes that they want. However it is possible for an artist to save a shape twice, or to save it at the wrong location in the pose space. For example, they might accidentally displace the skeleton before saving the pose, or some other error could occur.

The case where several poses are saved at the same location can be handled with regularization (section 8.2.1). Data that are clearly at the wrong location in the pose space can be treated with robust methods that deal with outliers. To briefly review, least squares is the ideal linear method when the outliers have an independent and identical Gaussian distribution. In this case, the probability of the data is proportional to product of the individual Gaussian errors and taking the log gives least squares. In the case where the errors have a more heavy-tailed distribution, leastsquares gives too much weight to the outliers, resulting in a bad fit. Robust methods down-weight the outliers by using an error measure other than the squared error. Common approaches are iteratively-reweighted least squares, which can approach a 1-norm (sum of absolute values), and least median of squares [191, 104].

The notion of "outlier" depends on the model that is used to fit the data. As a non-parametric model, RBF is capable of exactly fitting the 

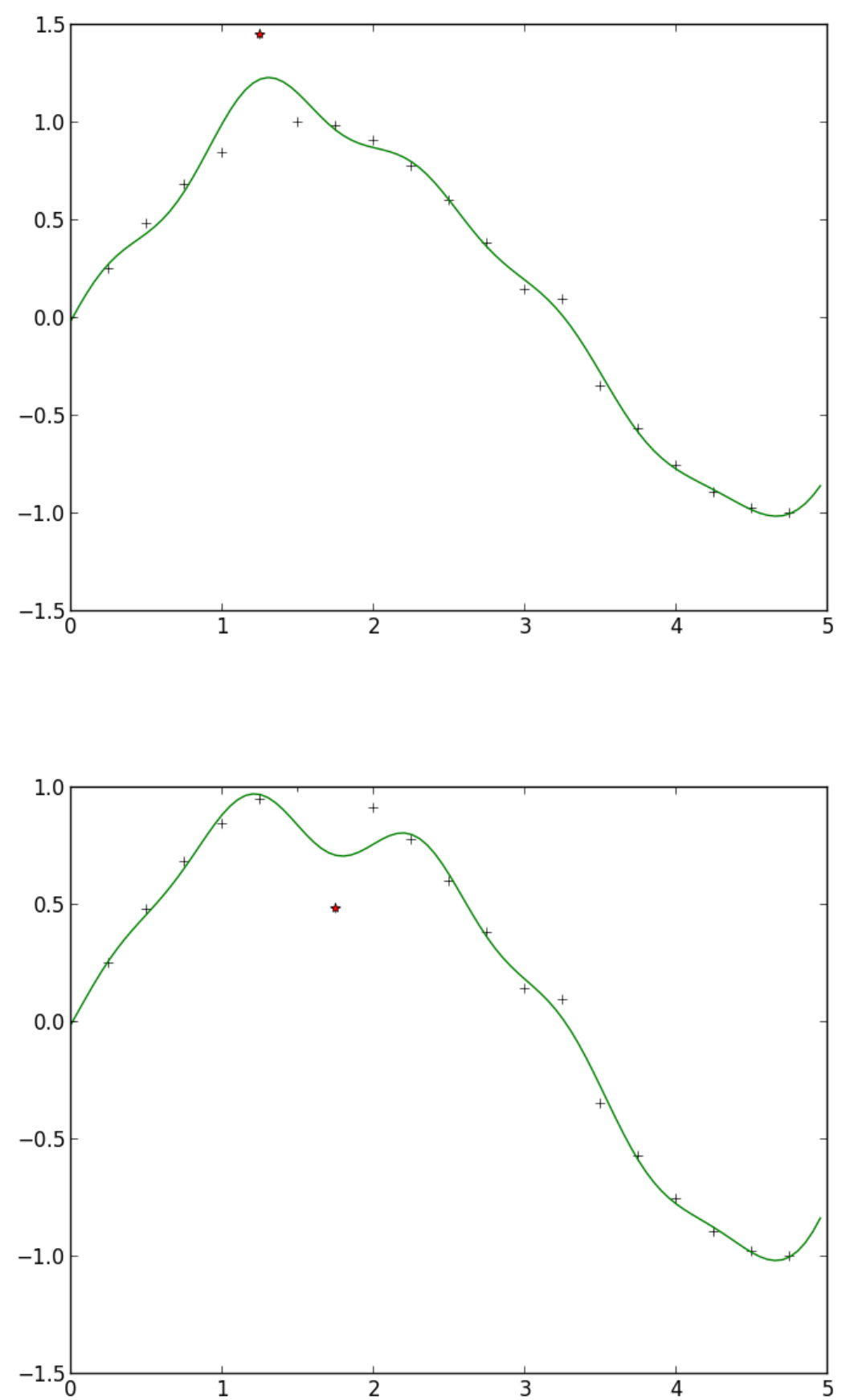

Figure 8.14: Two examples of a function norm-based approach to automatically identifying bad training data. The point marked with a red star is identified as an outlier. 
data, and thus standard methods (that assume fewer parameters than data points) are not applicable. How can outlying points be identified in this case? One possibility is to use the "function norm" $\mathbf{w}^{T} \mathbf{R} \mathbf{w}$ to identify the most inconsistent training point. In the case of a thin-plate spline, this norm corresponds to the thin-plate energy [77].

Specifically, we look for the point that, if removed, causes the largest reduction in this expression. $\mathbf{w}^{T} \mathbf{R w}$ is a sum of terms $w_{i} R_{i j} w_{j}$. A particular point contributes to the sum of these over a particular column (or row). Because of the symmetry of $\mathbf{R}$ we can consider the sum only over the corresponding row (or column). Note that the total contribution from a particular point involves both the corresponding row and the corresponding column, so the diagonal point is counted twice. Thus if summing only a column (or row) the diagonal point should be multiplied by $1 / 2$.

Figure 8.14 shows the results of this approach on two 1-dimensional test cases. While in these cases it finds what is evidently the most outlying points, in cases where the $\mathbf{R}$ matrix is poorly condition the interpolation is "wild" (Figure 8.13) and the chosen point is arguably not correct. Regularizing the inverse fixes this problem.

But this experiment revealed a simpler idea, which is to regularize, then pick the point that has the largest distance between itself and the regularized curve. The most outlying point seemed correct in all of a (small) number of test cases. Finding additional outliers requires removing the first-found point and re-solving with regularization again.

\subsubsection{Whence the RBF Kernel?}

The paper [139] did not describe the origin of the RBF kernel functions, and functions such as $r^{2} \log (r)$ are mysterious.

This section will present an intuitive explanation of the origins of the RBF kernel. The derivation involves only linear algebra, and the simplicity results from using a one dimensional example and assuming that the data 
can be represented on an underlying sampling grid of some resolution. For a slightly more formal derivation (involving variational calculus) see Appendix B.

\subsubsection{Green's functions: motivation}

Although Laplace and thin-plate interpolation is usually done by either sparse linear solves or relaxation/multigrid, it can also be done by radial basis interpolation, and this is faster if there are relatively few points to interpolate.

In these cases the kernel $R$ is the "Green's function of the corresponding squared differential operator". This section will explain what this means, and give an informal derivation for a simplified case. Specifically we're choosing a discrete one-dimensional setting with uniform sampling, so the problem can be expressed in linear algebra terms rather than with calculus.

The goal is find a function $f$ that interpolates some scattered data points $d_{k}$ while simultaneously minimizing the roughness of the curve. The roughness is measured in concept as

$$
\int|D f(x)|^{2} d x
$$

where $D$ is a "differential operator" $\left(D=\frac{d^{2}}{d x^{2}}\right)$ for example.

Whereas a function takes a single number and returns another number, an operator is something that takes a whole function and returns another whole function. The derivative is a prototypical operator, since the derivative of a function is another function. A differential operator is simply an operator that involves some combination of derivatives.

We are working in a discrete setting, so $D$ is a matrix (now denoted $\mathbf{D}$ ) that contains a finite-difference version of the operator. For example, for the second derivative, the (second order centered) finite difference is

$$
\frac{d^{2}}{d x^{2}} \approx \frac{1}{h^{2}}\left(f_{t+1}-2 f_{t}+f_{t-1}\right)
$$


This finite difference has the weight pattern $1,-2,1$, and for our purposes we can ignore the data spacing $h$ by considering it to be 1 , or alternately by folding it into the solved weights.

The finite difference version of the whole operator can be expressed as a matrix as

$$
\mathbf{D}=\frac{1}{h^{2}}\left[\begin{array}{cccccc}
-2 & 1 & & & & \\
1 & -2 & 1 & & & \\
& 1 & -2 & 1 & & \\
& & 1 & -2 & 1 & \cdots \\
& & & \ldots & &
\end{array}\right]
$$

(and again the $\frac{1}{h^{2}}$ can be ignored for some purposes). The discrete equivalent of the integral (8.2.5) is then $\|D f\|^{2}=\mathbf{f}^{T} \mathbf{D}^{T} \mathbf{D f}$.

Then our goal (of interpolating some scattered data while minimizing the roughness of the resulting function) can be expressed as

$$
\begin{aligned}
& \min _{\mathbf{f}}\|\mathbf{D f}\|^{2}+\boldsymbol{\lambda}^{T} \mathbf{S}(\mathbf{f}-\mathbf{d}) . \\
= & \min _{\mathbf{f}} \quad \mathbf{f}^{T} \mathbf{D}^{T} \mathbf{D} \mathbf{f}+\boldsymbol{\lambda}^{T} \mathbf{S}(\mathbf{f}-\mathbf{d}) .
\end{aligned}
$$

$\mathrm{S}$ is a "selection matrix", a wider-than-tall permutation matrix that selects elements of $\mathrm{f}$ that correspond to the known values in $\mathrm{d}$. So for example, if $f$ is a vector of length 100 representing a curve to be computed that passes through 5 known values, $\mathbf{S}$ will be $5 \times 100$ in size, with all zeros in each row except a 1 in the element corresponding to the location of the known value. The known value itself is in d, a vector of length 100 with 5 non-zero elements. $\boldsymbol{\lambda}$ is a Lagrange multiplier that enforces the constraint.

Now take the matrix/vector derivative of eq. (8.5) with respect to $f$,

$$
\frac{d}{d \mathbf{f}}=2 \mathbf{D}^{T} \mathbf{D f}+\mathbf{S}^{T} \boldsymbol{\lambda}
$$

and we can ignore the 2 (and a minus sign in the next step as well) by folding it into $\lambda$. 
If we know $\lambda$, the solution is then

$$
\mathbf{f}=\left(\mathbf{D}^{T} \mathbf{D}\right)^{-1} \mathbf{S}^{T} \boldsymbol{\lambda}
$$

- Although continuously speaking the differential operator is an "instantaneous thing", in discrete terms it is a convolution of the finite difference mask with the signal. Its inverse also has interpretation as a convolution.

- If $\mathbf{D}$ is the discrete version of $\frac{d}{d x}$ then $\mathbf{D}^{T} \mathbf{D}$ is the discrete Laplacian, or the "square" of the original differential operator. Likewise if $\mathbf{D}$ is the discrete Laplacian then $\mathbf{D}^{T} \mathbf{D}$ will be the discrete biharmonic, etc. $\left(\mathbf{D}^{T} \mathbf{D}\right)^{-1}$ is a discrete analogue of the Green's function of the squared differential operator.

In more detail, $\mathbf{D}^{T}$ is the discrete analogue of the adjoint of the derivative, and the latter is $-\frac{d}{d x}$ in the one-dimensional case [32]. The sign flip is evident in the matrix version of the operator: The rows of the original first derivative (forward or backward finite difference) matrix have $-1,1$ near the diagonal (ignoring the $\frac{1}{h}$ ). The transposed matrix thus has rows containing $1,-1$, or the negative of the derivative.

- $\mathbf{S}^{T} \boldsymbol{\lambda}$ is a vector of discrete deltas of various strengths (i.e. the discrete equivalent of Dirac deltas times a scaling function). In the example $\mathbf{S}^{T} \boldsymbol{\lambda}$ has five non-zero values out of 100 .

\subsubsection{Green's functions}

Earlier we said that the kernel is the "Green's function of the corresponding squared differential operator". A Green's function is a term from differential equations. A linear differential equation can be abstractly expressed as

$$
\mathrm{Df}=\mathbf{b}
$$


where $\mathbf{D}$ is a differential operator as before, $\mathbf{f}$ is the function we want to find (the solution), and $\mathbf{b}$ is some "forcing function". In the Green's function approach to solving a differential equation, the solution is assumed to take the form of a convolution of the Green's function with the right hand side of the equation (the forcing function). (We are skipping a detail involving boundary conditions). For linear differential equations the solution can be expressed in this form.

The Green's function $G$ is the "convolutional inverse" of the squared differential operator. It is the function such that the operator applied to it gives the delta function,

$$
D G=\delta
$$

While the theory of Green's functions is normally expressed with calculus, we will continue to rely on linear algebra instead. In fact, the appropriate theory has already been worked out in the previous subsection and merely needs to be interpreted.

Specifically, in Eq. 8.6), $\left(\mathbf{D}^{T} \mathbf{D}\right)^{-1}$ plays the role of the (discrete) Green's function of the squared differential operator. In discrete terms Eq. 8.7 is

$$
\mathbf{D}^{T} \mathbf{D}\left(\mathbf{D}^{T} \mathbf{D}\right)^{-1}=\mathbf{I}
$$

with the identity matrix rather than the $\delta$ function on the right hand side. $\mathbf{S}^{T} \boldsymbol{\lambda}$ is a sparse set of weighted impulses that is "convolved" (via matrix multiplication) with the Green's function. Thus we see that Eq. 8.6 is a discrete analogue to a Green's function solution to the original "roughness penalty" goal.

For simplicity this presentation assumed that $\mathbf{D}^{T} \mathbf{D}$ is invertible. However, if $\mathbf{D}$ is in fact a derivative operator, a pseudoinverse $\left(\mathbf{D}^{T} \mathbf{D}\right)^{+}$is required - the derivative is a prototypical example of an operation with a nullspace (the nullspace is the constant of integration). 


\subsubsection{Example derivation of a RBF kernel}

The preceding section provides an intuitive explanation of the idea of a Green's function, using only linear algebra. However, this approach does not give a closed form expression for the RBF kernel- it appears only as a matrix.

In Appendix B we derive a RBF kernel for a one-dimensional problem using variational calculus. The goal is to provide an example of what is involved, rather than to derive a particular kernel.

\subsection{Chapter Summary}

This chapter concludes Part two of the thesis, exploring nonlinear examplebased algorithms suitable for character body motion. The chapter extends published research by addressing the problem of bad data provided by the artist. A further contribution of the chapter and the associated appendix is a treatment of the origin and derivation of the RBF kernel. Most computer graphics publications simply quote known choices for the kernel, and even texts such as [45] do not describe how to derive a kernel (probably it is assumed to be straightforward for readers). 
178 CHAPTER 8. SCATTERED INTERPOLATION ALGORITHMS 


\section{Part III}

\section{Relationships}

The highest level of Bloom's taxonomy of learning [5] emphasizes the discovery of relationships between previously learned subjects. This final section of the thesis investigates relationships between several algorithms used in the example-based character animation approaches in the previous chapters. 


\section{Chapter 9}

\section{Relationships between various models}

Previous chapters have considered both linear and nonlinear approaches for example-based character animation. This final chapter is more theoretical, and investigates relationships between several techniques that have been discussed. Theories of learning [5] and knowledge often highlight the importance of discovering such relationships.

Several such relationships are also considered elsewhere in the thesis:

- Chapter 3 compares blendshape and PCA representations. These representations can be trivially interconverted, and blendshapes provide interpretability. PCA provides a data-driven regularizer, though we argue in Chapter 6 that it may be of limited use for face models.

- Chapter 8 describes the relationship between spline and Green's function formulations of interpolation, and provides an intuitive explanation of the Green's function idea.

In this chapter we consider these further relationships:

- RBF versus Gaussian Processes. We show that in computational terms these methods are very similar. They differ in their deterministic 
and probabilistic viewpoints, and in the assumption of what data is known in advance versus at runtime.

- RBF regression versus PCA. An Eigen-expansion of the RBF kernel function results in an expression that can be interpreted as a datadriven "superresolution" procedure for PCA.

- PCA versus Splines. For the purpose of representing faces, PCA and splines have complementary advantages and disadvantages. Motivated by this graphics/vision problem, we explore whether PCA and splines can be combined into a unified model.

\subsection{PCA, RBF, and Gaussian Processes}

To help describe the relationships between principal component analysis, radial basis function interpolation, and Gaussian processes, we will first briefly (re)introduce these techniques using a common notation. RBF interpolation is discussed in more detail in chapter 8 and appendix B. PCA is reviewed in section A.6.

Terminology. Vectors and matrices are indicated in bold font. $M(d, n, \mathbb{R})$ denotes the set of real matrices of size $d \times n$. The number of data points is denoted by $n, d$ is the dimensionality of a single data point in the case of PCA data, and $m$ is the number of eigenvectors retained in a PCA model. In order to suggest a relationship, related quantities are sometimes denoted with the same symbol. The usage is explained and is unique within each subsection. We will informally equate a radial basis function $R(\Delta)$ to a covariance. In so doing we consider covariances $C(x, y)=C(\|x-y\|)=$ $C(\Delta)$ corresponding to stationary processes. To simplify the presentation the mean of the process or data is assumed to be zero without loss of generality. We further use the covariance as the kernel in an RKHS. The reader should also distinguish continuous functions and the vectors and matrices generated by evaluating the continuous function at discrete locations. 
Principal Component Analysis. PCA represents data in a linear subspace obtained from the eigenvectors of the covariance matrix, estimated as $\mathbf{C} \approx \frac{1}{n} \mathbf{F F}^{T}$ where $\mathbf{F} \in M(d, n, \mathbb{R})$ is a matrix containing the data points $\mathbf{f}_{k} \in \mathbb{R}^{d}$ in its columns. We refer to a PCA "model" as

$$
\mathbf{f}=\mathbf{U} \mathbf{c}+\mathbf{m}
$$

where $\mathbf{f}$ is a vector representing the signal being modeled, $\mathbf{U} \in M(d, m, \mathbb{R})$ are eigenvectors of the covariance matrix $\mathbf{C}$ corresponding to the $m$ largest eigenvalues $(m \leq d), \mathbf{m} \in \mathbb{R}^{d}$ is the mean of the data points, and $\mathbf{c}$ are PCA "coefficients". In this model the data covariance

$$
\mathbf{C}=\mathbb{E}\left[(\mathbf{f}-\mathbf{m})(\mathbf{f}-\mathbf{m})^{T}\right]=\mathbb{E}\left[\mathbf{U} \mathbf{c c}^{T} \mathbf{U}^{T}\right]
$$

is replaced with the low rank approximation

$$
\mathbf{C} \approx \mathbf{U} \Lambda_{1: m} \mathbf{U}^{T}
$$

where $\boldsymbol{\Lambda}_{1: m} \in M(m, m, \mathbb{R})$ denotes the diagonal matrix formed from the largest eigenvalues.

Strictly speaking PCA is a valid model only in the case where the data is jointly Gaussian. Nevertheless, this approximate model is adequate in some applications. For example, a jointly Gaussian model of face proportions has been widely employed in computer vision [69] and even psychological theory [238]. While PCA is not considered a generative model, it is easy to synthesize new data having the same covariance as the observed data $\mathbf{f}_{k}$ by picking random coefficients $\mathbf{c}$ according to $c_{k}^{2} \sim N\left(0, \lambda_{k}\right)$.

To simplify notation, in the remainder of the chapter $\Lambda_{1: m}$ will be denoted simply as $\Lambda$. Also, the covariance matrix $\mathrm{C}$ should be understood as representing either the full rank covariance matrix or a low-rank approximation thereof. Most of the calculations are unchanged, although $\mathbf{C}^{-1}$ and $\Lambda^{-1}$ should be understood as pseudoinverses in the low-rank interpretation. 
RBF regression. $R B F$ regression at a location $\mathbf{p}$ has the form

$$
\hat{f}(\mathbf{p})=\sum_{k=1}^{n} a_{k} R\left(\left\|\mathbf{p}-\mathbf{p}_{k}\right\|\right)
$$

where $R()$ is a radial function situated at the $n$ training data locations $\mathbf{p}_{k}$. Assembling the data values to be interpolated in a vector $f$, the weights can be obtained from the linear system

$$
\mathbf{R a}=\mathbf{f}
$$

where $\mathbf{R}$ is the $n \times n$ matrix consisting of the radial basis function evaluated at all pairs of data locations, $\mathbf{R}_{r, c}=R\left(\left\|\mathbf{p}_{r}-\mathbf{p}_{c}\right\|\right)$. This describes the case of regression with a positive definite radial kernel $R$. Other RBF kernels are conditionally positive definite and require solving a block matrix system [9]. In matrix-vector notation the regression can be written

$$
\hat{f}(\mathbf{p})=\mathbf{r}^{T} \mathbf{R}^{-1} \mathbf{f}
$$

where $\mathbf{r} \equiv \mathbf{r}_{\mathbf{p}}$ is the vector of the kernel evaluated at the distance between the evaluation location $\mathbf{p}$ and all the training locations $\mathbf{p}_{k}$, i.e.,

$$
\mathbf{r}=\left[R\left(\left\|\mathbf{p}-\mathbf{p}_{1}\right\|\right), R\left(\left\|\mathbf{p}-\mathbf{p}_{2}\right\|\right), R\left(\left\|\mathbf{p}-\mathbf{p}_{3}\right\|\right), \cdots, R\left(\left\|\mathbf{p}-\mathbf{p}_{n}\right\|\right)\right]
$$

Note that the position $\mathbf{p}$ is arbitrary.

Gaussian process regression. A discrete Wiener filter estimates the signal $\hat{f}(\mathbf{p})$ at a location $\mathbf{p}$ as a linear weighted sum of the data $\mathbf{f}$ at previous or surrounding locations,

$$
\hat{f}(\mathbf{p})=\mathbf{w}^{T} \mathbf{f} .
$$

The orthogonality principle [176] states that the error of the optimal estimator is orthogonal in expectation to the data:

$$
\mathbb{E}\left[\left(f(\mathbf{p})-\mathbf{w}^{T} \mathbf{f}\right) \mathbf{f}^{T}\right]=0
$$


or equivalently

$$
\mathbb{E}\left[f(\mathbf{p}) \mathbf{f}^{T}\right]=\mathbf{w} \mathbb{E}\left[\mathrm{ff}^{T}\right] .
$$

$\mathbb{E}\left[\mathrm{ff}^{T}\right]=\mathbf{C}$ is the covariance of a zero-mean stationary process and $\mathbb{E}\left[f(\mathbf{p}) \mathbf{f}^{T}\right]=$ $\mathbf{r}^{T}$ is the cross covariance between the signal at the location $\mathbf{p}$ and at the data points $f\left(\mathbf{p}_{k}\right) \equiv f_{k}$ for $k=1, \cdots, n$. This gives a linear system $\mathbf{C w}=\mathbf{r}$ that can be used to solve for the weight vector w. Substituting in Equation (9.3) gives the estimator

$$
\hat{f}(\mathbf{p})=\mathbf{r}^{T} \mathbf{C}^{-1} \mathbf{f} .
$$

The variance of the estimator is

$$
\begin{aligned}
\mathbb{E}\left[\left(f-\mathbf{w}^{T} \mathbf{f}\right)^{2}\right] & =\sigma^{2}+\mathbb{E}\left[-2 f \mathbf{w}^{T} \mathbf{f}+\mathbf{w}^{T} \mathbf{f} \mathbf{f}^{T} \mathbf{w}\right] \\
& =\sigma^{2}-2 \mathbf{w}^{T} \mathbb{E}[f \mathbf{f}]+\mathbf{w}^{T} \mathbf{C} \mathbf{w}
\end{aligned}
$$

where $\sigma^{2}$ is the variance of the (stationary) process, and $f \equiv f(\mathbf{p})$ is written for brevity. Substituting the cross-covariance $\mathbf{r}$ and the weights $\mathbf{w}=\mathbf{C}^{-1} \mathbf{r}$, we have

$$
\begin{aligned}
& =\sigma^{2}-2 \mathbf{w}^{T} \mathbb{E}[f \mathbf{f}]+\mathbf{r}^{T} \mathbf{C}^{-1} \mathbf{C C}^{-1} \mathbf{r} \\
& =\sigma^{2}-\mathbf{r}^{T} \mathbf{C}^{-1} \mathbf{r} .
\end{aligned}
$$

Equation (9.4) and this expression for the variance have the same form as the mean and variance in Gaussian process (GP) regression [199, 2.19].

\subsection{RBF versus Gaussian Processes}

The RBF and Gaussian Process (GP) regression initially seem quite different. The GP expression for the mean is a (linear) weighted sum of the data, whereas RBF is a weighted sum of a (nonlinear) kernel indexed by the distances between the data.

However, if we equate the kernel with the covariance, these models actually do the same computation, as can be seen from equations (9.2) 
and (9.4). This correspondence requires simultaneous generalization of the RBF kernel to non-radially-symmetric form and a restriction to valid (i.e. positive-(semi)definite) covariances. The relationship is summarized in table 9.1 at the end of the chapter.

\subsubsection{Outlook}

This partial equivalence between RBF and GP regression may allow particular computational techniques to be transferred from one method to the other. On the other hand, despite their computational similarity, the two methods have very different assumptions and usage.

One difference between these approaches is what data is assumed known in advance. Denoting the precomputed part with parentheses, we have:

$$
\text { RBF: } \quad \hat{f}_{0}=\left(\mathbf{f}^{T} \mathbf{R}^{-1}\right) \mathbf{r}
$$

In other words, the RBF typically assumes that the data to be interpolated is known in advance, but the locations at which it will be interpolated are not known (at the time the weights are computed). The GP formulation need not assume this.

Assuming the symmetry of the covariance (equivalently the RBF kernel), in concept one could employ a Gaussian process code for RBF regression simply by flipping the $\mathbf{r}$ and $\mathbf{f}$ arguments provided to the code. In practice this usually will not work, because the $\mathbf{r}$ vector is generally not provided, but rather is computed through the covariance.

In the case of a vector-valued function, RBF interpolation simply stacks a number of scalar RBF systems. These systems share the same $\mathbf{R}$ matrix, but have different weight vectors. However, for vector valued data the expectation $E\left[f_{a} f_{b}\right]$ is itself a matrix so the covariance is a tensor, whereas $\mathbf{R}$ is always a matrix. The RBF kernel is isotropic by definition, not so for the covariance in multi-dimensions. 


\subsection{A Superresolution Extension of PCA}

Principal component analysis (PCA) involves a signal that is sampled at some arbitrary but fixed and finite set of locations. Radial Basis Function (RBF) regression interpolates a-priori known data to arbitrary locations as a weighted sum of a (radial) kernel function centered at the data points. In the previous section we showed that if the RBF kernel is equated to the covariance, RBF and Gaussian Process (GP) models perform a similar computation.

Building on the RBF-GP equivalence, we next show that if the data covariance is known (or can be estimated), an RBF-inspired regression can provide data-driven interpolation of given data. This procedure can alternately be interpreted as a superresolution ${ }^{1}$ extension of eigenvector (principal component) data models, as signal sampling (function evaluation) in a discrete reproducing kernel Hilbert space (RKHS) generated by the data covariance, or as an elementary Gaussian process model in which the observations have a low-rank representation.

\subsubsection{Superresolution}

In the remainder of this section we first describe the superresolution procedure. We show that the procedure has the representer of evaluation and reproducing kernel properties of an RKHS, albeit in discrete rather than continuous form. Finally, we show a computational experiment using the superresolution procedure.

We start from the matrix-vector representation of RBF regression, (9.2). We will equate $R$ to a covariance function $C$, which is a generalization to non-radially symmetric kernels. "Oriented" flavors of RBF regression have previously appeared in the literature [76, [52]. Since the matrix $\mathbf{R}$ is symmetric, it has a low-rank approximation $\mathbf{U} \boldsymbol{\Lambda} \mathbf{U}^{T}$. Substituting this

\footnotetext{
1 "Superresolution" denotes the ability to estimate the signal "in between" the data samples, i.e. in our case at a finer scale than the spacing of the data used in the PCA.
} 
eigenexpansion, the regression takes the form

$$
\hat{f}(\mathbf{p})=\mathbf{r}^{T} \mathbf{C}^{-1} \mathbf{f}=\mathbf{r}^{T} \mathbf{U} \boldsymbol{\Lambda}^{-1} \mathbf{U}^{T} \mathbf{f}
$$

Denoting $\mathbf{c} \equiv \boldsymbol{\Lambda}^{-1} \mathbf{U}^{T} \mathbf{f}$, Equation (9.5) can be interpreted as incorporating the results of a PCA-like representation Uc involving the data covariance eigenvectors $\mathbf{U}$ (we again assume the data mean is zero):

$$
\begin{aligned}
\hat{f}(\mathbf{p}) & =\mathbf{r}^{T} \mathbf{U}\left(\boldsymbol{\Lambda}^{-1} \mathbf{U}^{T} \mathbf{f}\right) \\
& =\mathbf{r}^{T} \mathbf{U} \mathbf{c}
\end{aligned}
$$

The discrete principal component model Equation (9.1) involves data that is sampled at some arbitrary but fixed and finite set of locations. Equation (9.6) extends this by premultiplying with $r$. Recall that $r$ can be interpreted as a vector of cross-covariances between the location $\mathrm{p}$ at which the the regression is evaluated and the locations of the data points $\mathbf{p}_{k}$,

$$
\mathbf{r}=C(\mathbf{p}, \cdot)=\left[C\left(\left\|\mathbf{p}-\mathbf{p}_{1}\right\|\right), C\left(\left\|\mathbf{p}-\mathbf{p}_{2}\right\|\right), C\left(\left\|\mathbf{p}-\mathbf{p}_{3}\right\|\right), \cdots, C\left(\left\|\mathbf{p}-\mathbf{p}_{n}\right\|\right)\right]
$$

If the covariance function $C(\boldsymbol{\Delta})$ is known for all offsets $\Delta$ then the data estimate (9.6) can be evaluated at arbitrary locations, effectively providing a covariance-driven superresolution.

We next sketch an argument that function generated by the superresolution estimate (9.6) in fact has covariance $C$. In this section let $\mathbf{r}_{i}$ denote the cross-covariance (9.7) evaluated at the location $\mathbf{p}_{i}$ (rather than at an arbitrary location). The covariance of the estimated signal is:

$$
\begin{aligned}
\mathbb{E}\left[f\left(\mathbf{p}_{i}\right) f\left(\mathbf{p}_{j}\right)\right] & =\mathbb{E}\left[\mathbf{r}_{i}^{T} \mathbf{C}^{-1} \mathbf{f} \mathbf{f}^{T} \mathbf{C}^{-1} \mathbf{r}_{j}\right] \\
& =\mathbf{r}_{i}^{T} \mathbf{C}^{-1} \mathbf{C} \mathbf{C}^{-1} \mathbf{r}_{j} \\
& =\mathbf{r}_{i}^{T} \mathbf{C}^{-1} \mathbf{r}_{j} \\
& =\mathbf{C}_{i, j}
\end{aligned}
$$

(For the last step note that $\mathbf{r}_{i}$ is the $i$ th row of $\mathbf{C}$, hence multiplying by $\mathbf{C}^{-1}$ gives $\mathbf{e}_{i}$, the indicator vector with 1 in the $i$ th location, otherwise zero). 

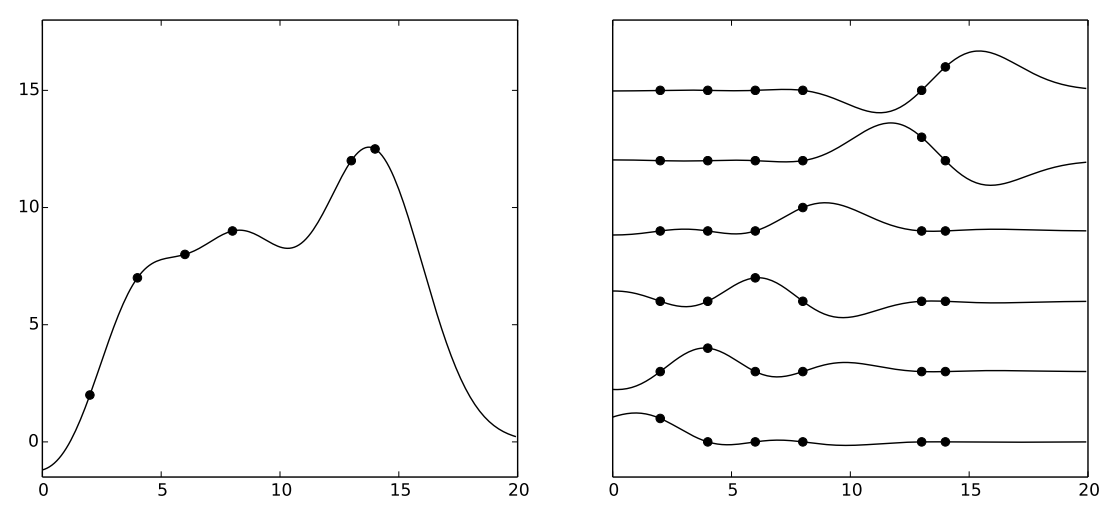

Figure 9.1: Left: Radial basis interpolation of six points using a Gaussian kernel. Right: equivalent kernels for each of the data points, offset vertically for visibility.

\subsubsection{Equivalent kernel}

Note that Equation (9.4) is more commonly interpreted in a different way. In this interpretation

$$
\begin{aligned}
\hat{f}(\mathbf{p}) & =\left(\mathbf{r}^{T} \mathbf{C}^{-1}\right) \mathbf{f} \\
& =\mathbf{b}(x)^{T} \mathbf{f} .
\end{aligned}
$$

Here $\mathbf{b}(x)=\mathbf{C}^{-1} \mathbf{r}$ is the vector of equivalent kernels [96] evaluated at location $x$. The individual kernels $\mathbf{b}_{k}(x)$ are one at the location $x_{k}$ of the $k$ th data point, and zero at the locations of the other data points (Figure 9.1).

\subsubsection{Interpretation as evaluation in an RKHS}

In this section we show that Equation (9.5) resembles function evaluation in an RKHS corresponding to the covariance $\mathbf{C}$. 


\section{Representer of evaluation}

The kernel in an RKHS acts similarly to a delta function, providing function evaluation at a particular location under the inner product:

$$
\langle f, K(\cdot, x)\rangle_{C}=f(x)
$$

In our discrete case we propose that

$$
\mathbf{f}^{T} \mathbf{C}^{-1} \mathbf{r}_{x}=f(j)
$$

with $\mathbf{r}_{x}$ defined as the vector of $K(\cdot, x)$ evaluated at the data points. In the case of where $x$ is one of the data locations $\mathbf{p}_{j}$,

$$
\mathbf{r}_{x}=\mathbf{C e}_{j}
$$

( $\mathbf{e}_{m}$ is the indicator vector). Then, substituting $\mathbf{r}_{x}$, we have

$$
\mathbf{f}^{T} \mathbf{C}^{-1} \mathbf{C e}_{j}=f(j) .
$$

\section{Reproducing property}

Similarly,

$$
\begin{gathered}
\langle K(\cdot, x), K(\cdot, y)\rangle=K(x, y) \\
\approx\left\langle\mathbf{e}_{x}^{T} \mathbf{C}, \mathbf{C} \mathbf{e}_{y}\right\rangle_{C}=\mathbf{e}_{x}^{T} \mathbf{C ~} \mathbf{C}^{-1} \mathbf{C} \mathbf{e}_{y}=\mathbf{e}_{x}^{T} \mathbf{C} \mathbf{e}_{y}=\mathbf{C}_{x, y} .
\end{gathered}
$$

\section{Inner Product}

The analogy between (9.8) and (9.9) requires an inner product of the form

$$
\left\langle\mathbf{v}_{1}, \mathbf{v}_{2}\right\rangle_{C}=\mathbf{v}_{1}^{T} \mathbf{C}^{-1} \mathbf{v}_{2} .
$$

This can be motivated as follows: in the continuous case, the reproducing kernel has the (Mercer) expansion:

$$
\mathbf{C}(x, y)=\sum_{k=1}^{\infty} \lambda_{k} \mathbf{u}_{k}(x) \mathbf{u}_{k}(y)
$$


A signal is also representable in terms of the eigenvectors of the kernel:

$$
f(x)=\sum_{k=1}^{\infty} c_{k} \mathbf{u}_{k}(x) .
$$

The inner product is defined to be

$$
\left\langle\sum_{k=1}^{\infty} c_{k} \mathbf{u}_{k}(x), \sum_{k=1}^{\infty} d_{k} \mathbf{u}_{k}(x)\right\rangle_{C}=\sum_{k=1}^{\infty} \frac{c_{k} d_{k}}{\lambda_{k}}
$$

giving the norm $\sqrt{\sum_{k=1}^{\infty} \frac{c_{k}^{2}}{\lambda_{k}}}$.

In practice in the discrete case the summation is finite. The squared norm can be written in matrix-vector form

$$
\sum_{k=1}^{m} \frac{c_{k}^{2}}{\lambda_{k}}=\mathbf{c}^{T} \boldsymbol{\Lambda}^{-1} \mathbf{c}
$$

and re-expressed in terms of the kernel as

$$
\sum_{k=1}^{m} \frac{c_{k}^{2}}{\lambda_{k}}=\mathbf{f}^{T} \mathbf{U} \boldsymbol{\Lambda}^{-1} \mathbf{U}^{T} \mathbf{f}=\mathbf{f}^{T} \mathbf{C}^{-1} \mathbf{f} .
$$

We will take the kernel as the covariance matrix, and for simplicity consider only the case of positive definite covariances. In this case all the eigenvalues are positive real and the norm is valid.

\subsubsection{Computational Experiment}

The superresolution estimate (9.6) requires supplying or estimating a covariance matrix. In some cases the form of the covariance matrix is known or assumed. For example, the use of the Discrete Cosine Transform (DCT) in image compression is motivated by the choice of $\mathbf{C}_{r, c}=\rho^{|r-c|}, \rho \approx 0.9$ as a generic covariance for images [198]. Estimating a nonparametric covariance matrix from data requires care if the number of data points is not sufficient to reliably estimate the $n(n+1) / 2$ parameters of the covariance 
matrix, and is a subject of continuing research [193]. Although the covariance must be estimated, even an approximate covariance might result in better interpolation than would be obtained using generic spline interpolation.

There are known algorithms for generating multivariate normal random vectors with a specified covariance [94]. One simple algorithm is obtained by considering the covariance of a linearly transformed random vector $\mathbf{x}=\mathbf{T n}$ where $\mathbf{n}$ is an uncorrelated normal random vector (i.e. having an identity covariance matrix):

$$
\mathbf{C}_{x}=\mathbb{E}\left[\mathbf{T n n}^{T} \mathbf{T}^{T}\right]=\mathbf{T T}^{T}
$$

Thus, if the transform $\mathbf{T}$ is a "square root" of a desired covariance matrix $\mathbf{C}=\mathbf{T} \mathbf{T}^{T}$, then $\mathbf{x}$ will have covariance $\mathbf{C}$ (the decomposition is not unique). $\mathbf{T}$ can be obtained using the Cholesky decomposition if the covariance is non singular. This algorithm is limited to generating vectors $\mathbf{x}$ with a fixed number of samples at offsets corresponding to those that generated the covariance matrix, whereas our procedure can generate the signal at arbitrary locations.

In Figures 9.2 9.4 we simply select a covariance for the purpose of illustration, whereas Figure 9.5 uses a covariance estimated from real data.

Figure 9.2 shows an experiment using the covariance $C(\Delta)=\exp \left(-(15 \Delta)^{2}\right)$. The low-resolution points are interpolated exactly.

Figure 9.3 uses the oscillatory kernel $C(\Delta)=\exp \left(-\Delta^{2}\right) \cos (18 \Delta)$ to interpolate randomly sampled data points. This figure illustrates an important difference between this form of superresolution and spline interpolation: while spline interpolation is generic (data agnostic) and minimizes a form of curvature, the superresolution is based on the covariance and can potentially emphasize curvature. In this case the chosen kernel forces wild oscillation.

Figure 9.4 shows hole filling or "inpainting", in which a contiguous range of data are missing and filled in. 
Figure 9.5 shows inpainting of an approximately periodic signal obtained from motion capture of a joint angle (x-rotation of left tibia) from a walking motion. The data for this example was obtained from Subject \#2 in 67].

\subsubsection{Outlook}

Radial basis functions, principal component analysis, and Gaussian processes are widely used in computer graphics and computer vision. Particular applications include reconstruction of scanned data [51], motion capture from single-cameras [206], character animation [216], and many others. As each technique is founded on pairwise relationships expressed through a two-argument kernel or covariance function, it is not surprising that relationships between these techniques can be discovered. We illustrate the value of exploring these relationships through the derivation of a data-driven superresolution procedure.

\subsection{Exploring the relation between PCA and Lapla- cian Splines}

Principal component analysis (PCA) and Laplacian splines (LS) are popular approaches to modeling shapes in medical image analysis, computer vision, computer graphics, and other fields. PCA and LS are frequently employed as prior models to regularize inverse problems. In the context of face fitting, for example, both PCA [208] and LS approaches [65] have been employed. These distinct approaches have complementary advantages and weaknesses. Here we show that PCA and LS can be represented in (nearly) a common form, thereby revealing a relationship between these models.

We denote by "Laplacian splines" the family of interpolation schemes that minimize the integral of a squared derivative operator on the func- 

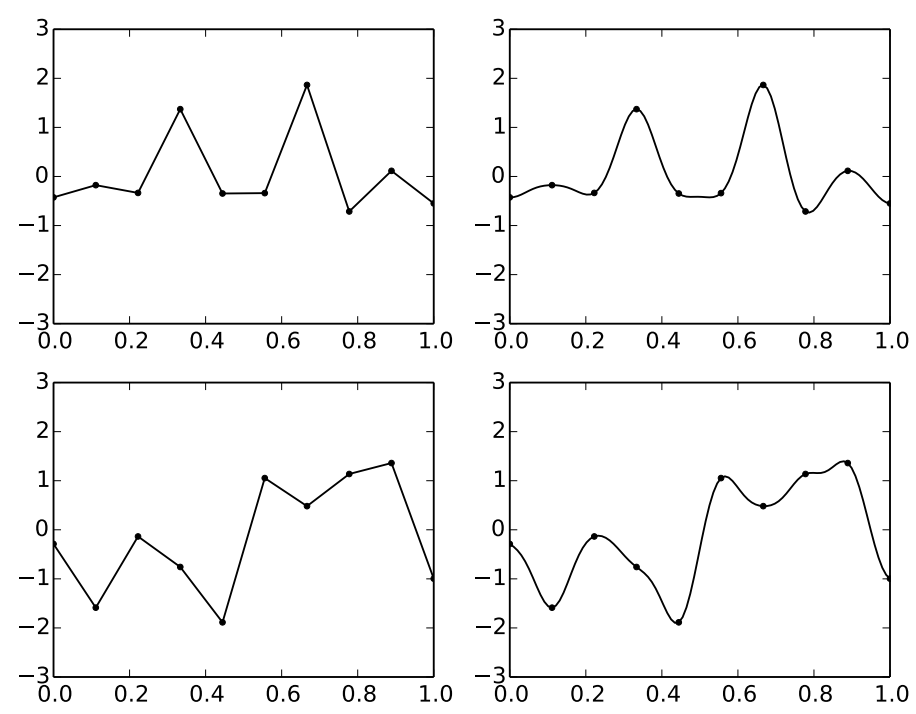

Figure 9.2: Left column: example signals synthesized with covariance $\exp \left(-(15 \Delta)^{2}\right)$. Right column: corresponding superresolution signals. The indicated points are interpolated exactly. 

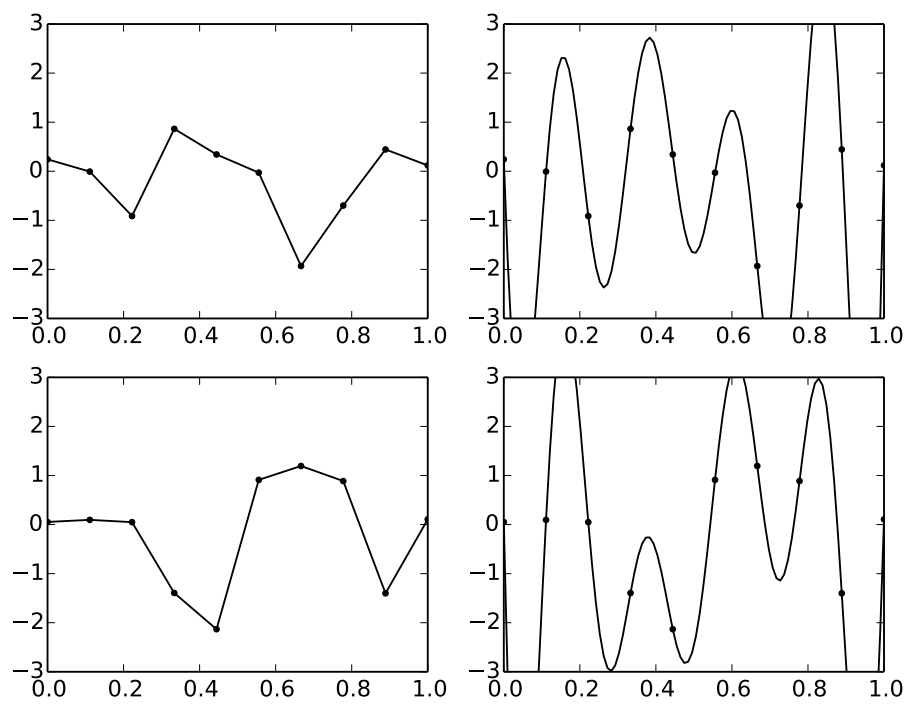

Figure 9.3: Left column: signals synthesized with kernel $\cos (18 \Delta) \exp \left(-\Delta^{2}\right)$. Right column: corresponding superresolution signals. The indicated points are interpolated exactly. This kernel illustrates that the superresolution procedure is different from spline interpolation. In this case it is adding detail in the form of oscillation. 

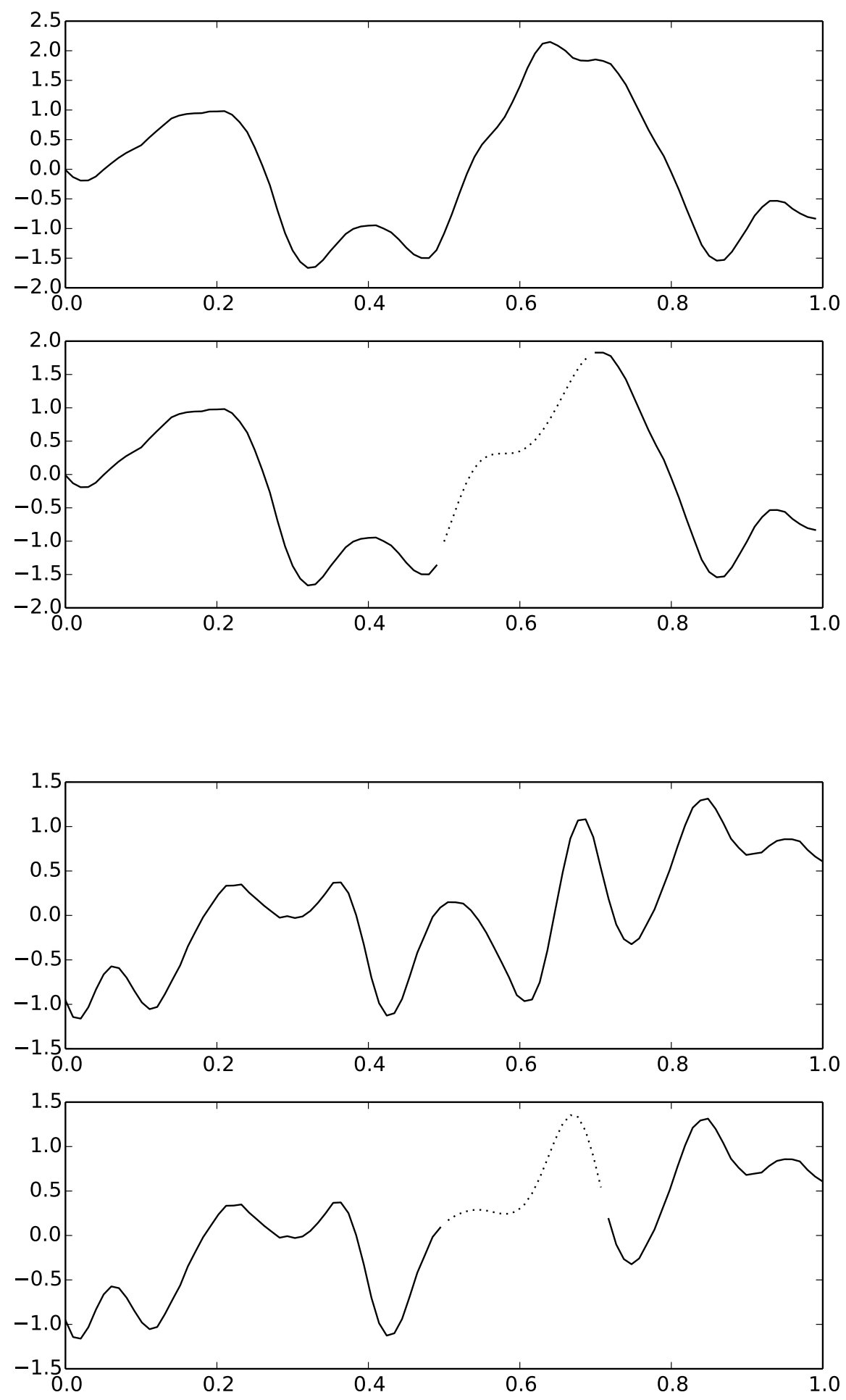

Figure 9.4: Inpainting of signals synthesized from the covariance $\exp \left(-(15 \Delta)^{2}\right)$. The top plot in each subfigure is the original signal; the bottom plot is signal with an omitted section estimated. 


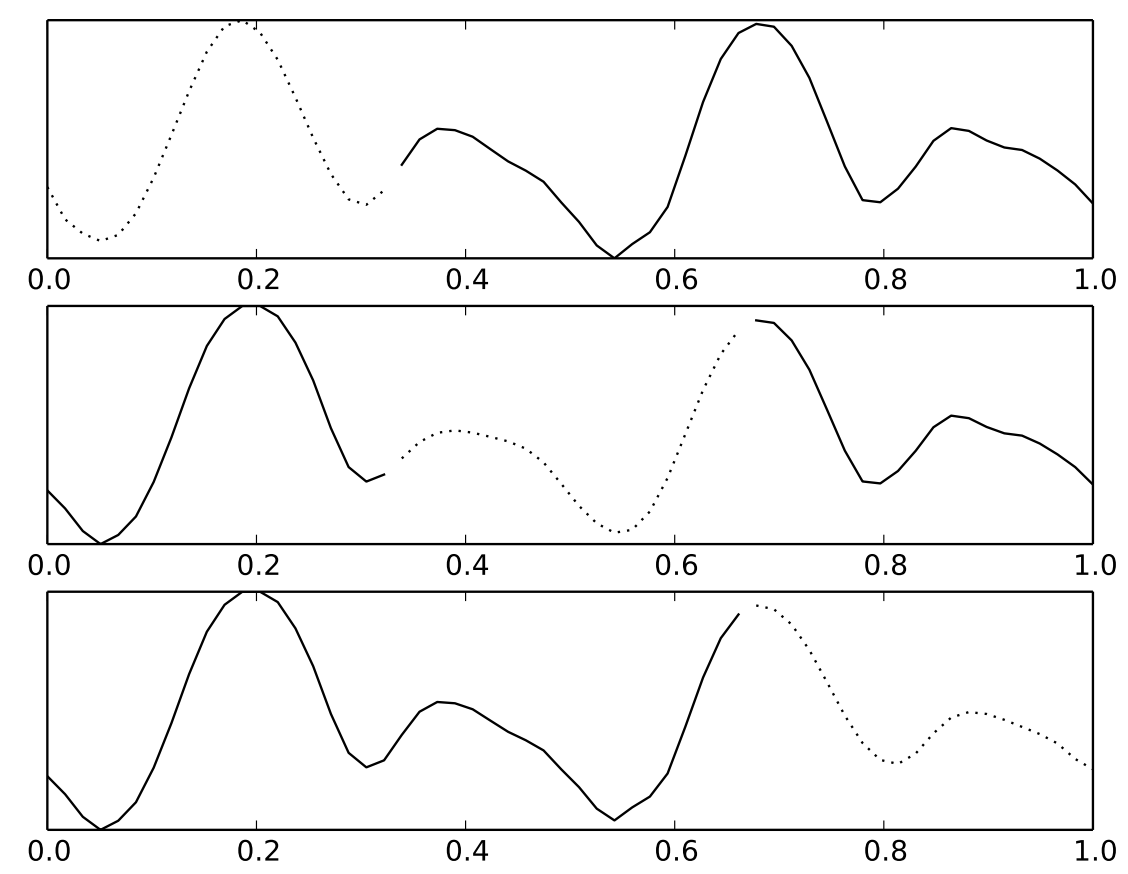

Figure 9.5: Inpainting of a joint angle signal from motion capture of a walking motion [67]. Each subplot shows one third of the signal omitted and estimated. The covariance is estimated from the original signal (Figure 9.6). 


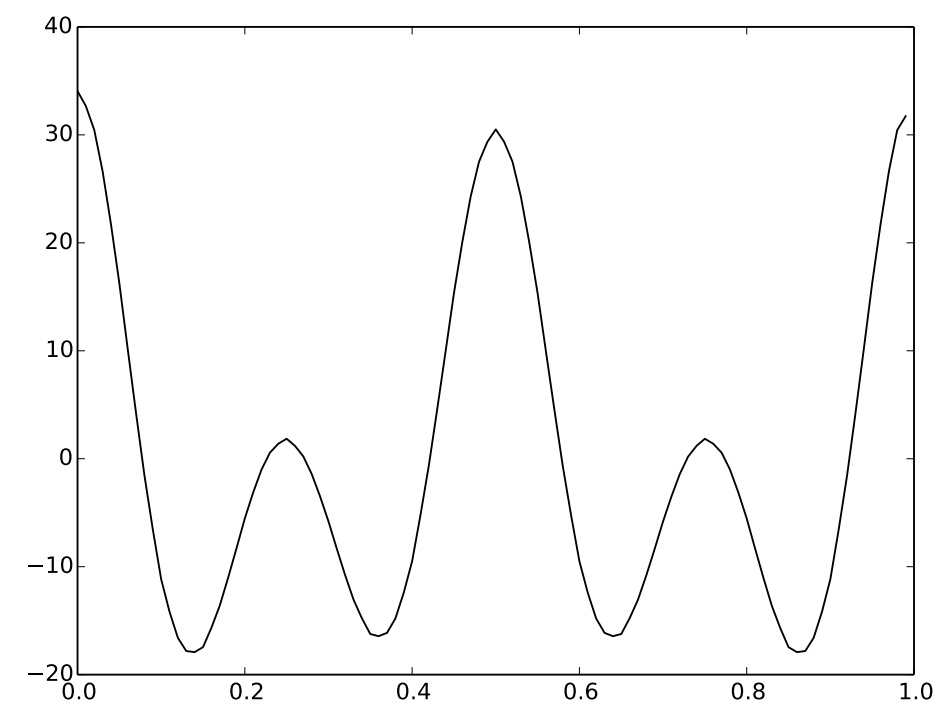

Figure 9.6: Covariance function estimated from the data used in Figure 9.5.

tion, e.g. membrane (Laplace) interpolation, biharmonic interpolation and thin-plate splines. These methods can interpolate arbitrary data, however they do not embody any statistics of the particular data, preferring only that the interpolation be as smooth (in the sense of minimising the integrated squared derivative) as possible. These splines are commonly used for shape registration in medical imaging and computer vision [77, 133, 36]. In computer graphics they have been used for surface deformation, implicit surface modeling, and other purposes [39, 234].

PCA has the advantage that it captures the Gaussian statistics of a class of shapes and thus has some "knowledge" of what is (and is not) a reasonable shape from that class. On the other hand, PCA is usually employed as a low rank model, and thus it cannot exactly represent all possible shapes. This is appropriate in cases where there is measurement noise in the shape data acquisition. However in some applications (for example in movie visual effects and computer graphics) we assume that the data is high- 
quality and has little or no noise. A particular example is facial motion capture for movie applications [189, 190]. The difference in facial position between two mental states (perhaps "calm" and "contempt") may be quite small, $1 \mathrm{~mm}$ or less. Thus the facial motion capture process requires that very accurate data be obtained. A PCA model obtained during an initial motion capture will not exactly span the data captured in subsequent capture sessions, due to differing placement of motion capture markers or other reasons, but accurate tracking of the face position is nevertheless required.

The complementary strengths and limitations of the PCA and LS approaches are illustrated in Fig. 9.7 and summarized in this table:

\begin{tabular}{|c|c|c|}
\hline & low rank PCA & Laplacian spline \\
\hline \hline “knowledge" of the data & yes & no \\
\hline can fit data exactly & no & yes \\
\hline
\end{tabular}

\subsubsection{PCA}

A PCA model is of the form

$$
\mathbf{f}=\mathbf{U} \mathbf{c}+\mathbf{m}
$$

where $\mathbf{U}$ is a matrix whose columns are the eigenvectors of the data covariance matrix, $\mathbf{c}$ are coefficients, and $\mathbf{m}$ is the mean shape (mean data vector). In our discussion we take $\mathbf{f}$ as a discrete, uniformly sampled, and one-dimensional signal for simplicity. PCA is usually applied to multidimensional data by "vectorizing" the data into one-dimensional form by scanning the multiple dimensions in some arbitrary but consistent order, though intrinsically multidimensional variants of PCA have also been proposed [75]. We also take the mean to be zero without loss of generality.

Most commonly, the PCA model discards all eigenvectors corresponding to small eigenvalues, with the assumption that these variances are 
noise, or alternately that a simpler model that captures most but not all of the variation is preferable.

A form that will expose the relationship to LS is

$$
\min _{\mathbf{c}}\|\mathbf{c}\|_{\Lambda}^{2}+\boldsymbol{\lambda}^{T} \mathbf{S}(\mathbf{U} \mathbf{c}-\mathbf{d})
$$

i.e. minimize a norm on the coefficients subject to interpolating some (sparse) data using the PCA model. Here $\mathbf{d}$ is a vector of data points, with zero (or any other value) where no data is available, and $\mathbf{S}$ is selects only the rows of $\mathbf{U} \mathbf{c}-\mathbf{d}$ corresponding to the available data (i.e. $\mathbf{S}_{r, c}=1$ selects the $c$ th row, zeros elsewhere). $\lambda$ is a Lagrange multiplier vector (distinguish from $\Lambda$, the diagonal matrix of eigenvectors $\lambda_{k}$ ).

Because the expectation $\mathbb{E}\left[\mathbf{c c}^{T}\right]=\mathbf{U}^{T} \mathbb{E}\left[\mathrm{ff}^{T}\right] \mathbf{U}=\mathbf{U}^{T} \mathbf{C U}=\boldsymbol{\Lambda}$ with $\mathbf{C}$ the covariance, the expectation squared of an individual coefficient is $c_{k}^{2} \sim$ $\lambda_{k}$. Thus the standard choice for the prior \|\|$_{\Lambda}$ is $\sum c_{k}^{2} / \lambda_{k}$. This prior is commonly used in applications, e.g. [214, 11] and many others. We rewrite the PCA fitting problem as

$$
\min _{\mathbf{c}} \mathbf{c}^{T} \boldsymbol{\Lambda}^{-1} \mathbf{c}+\boldsymbol{\lambda}^{T} \mathbf{S}(\mathbf{U} \mathbf{c}-\mathbf{d})
$$

\subsubsection{Laplacian Splines}

Laplacian splines minimize an energy $\int_{\Omega}\|\mathbf{L} \mathbf{f}\|^{2} d \mathbf{x}$ (where $\mathbf{L}$ is a derivative operator such as the gradient or Laplacian) subject to interpolating specified constraints. Thin plate splines are related, minimizing an energy

$$
\iint\left(f_{x x}^{2}+2 f_{x y}^{2}+f_{y y}^{2}\right) d x d y
$$

that includes additional cross terms.

The constraint of interpolating the available data can be expressed as

$$
\min _{\mathbf{f}}\|\mathbf{L f}\|^{2}+\boldsymbol{\lambda}^{T} \mathbf{S}(\mathbf{f}-\mathbf{d})
$$



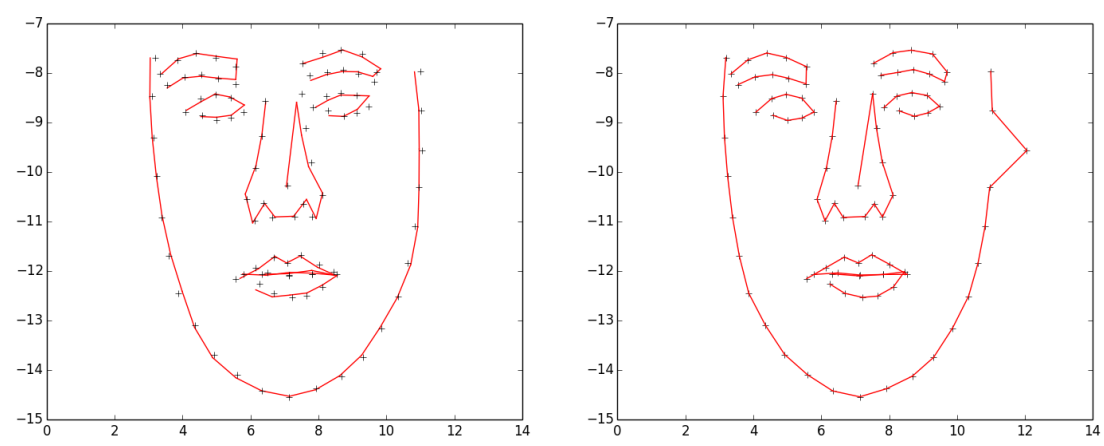

Figure 9.7: Left: A reduced-rank PCA model does not exactly fit the data. In this figure the model points are connected with lines for visualization. Right: a spline (in this case a linear spline) fits the data exactly, but does not exclude unreasonable data such as the displaced point in this figure.

where $\mathbf{L}$ is a discrete approximation to a derivative operator. Again we choose a one-dimensional and uniformly sampled version of the problem to simplify the discussion. In this case the Laplacian has the form

$$
\text { const }\left[\begin{array}{rrr}
2-1 & 0 & 0 \ldots \\
-1 & 2-1 & 0 \ldots \\
0-1 & 2-1 \ldots \\
\ldots &
\end{array}\right]
$$

\subsubsection{Relating PCA and Laplacian Splines}

The desired signal $\mathbf{f}$ can be represented with a PCA or other orthogonal basis as $\mathbf{f}=$ Vc. Substituting this into (9.11) gives

$$
\min _{\mathbf{c}} \mathbf{c}^{T} \mathbf{V}^{T} \mathbf{L}^{2} \mathbf{V} \mathbf{c}+\boldsymbol{\lambda}^{T} \mathbf{S}(\mathbf{V} \mathbf{c}-\mathbf{d})
$$

If $\mathbf{V}$ are the eigenvectors of $\mathbf{L}$, then $\mathbf{V}^{T} \mathbf{L}^{2} \mathbf{V}$ is diagonal and (9.10, (9.13) are in the same form. This might initially suggest that $\mathbf{L}^{2}$ plays the role 
of the covariance matrix in PCA. This interpretation is not entirely satisfactory in that, while the second difference (Laplacian) matrix is positive semidefinite, it reflects a covariance that decays very quickly. However, the eigenvalues of a matrix are also the eigenvalues of its inverse. This suggest that $\mathbf{L}^{2}$ may play the role of the inverse covariance, i.e. the precision matrix.

Can $L^{2}$ be interpreted as a (pseudo) inverse covariance? Several authors have noted a relationship of this form [86, 199] but without elaboration. To motivate this interpretation we note the following:

1. The matrix $\left[C_{r, c}\right]=\rho^{|r-c|}$ is known as the Kac-Murdock-Szego matrix, and for $\rho \neq 1$ has the inverse [78]

$$
\frac{1}{1-\rho^{2}}\left[\begin{array}{ccccc}
1 & -\rho & 0 & 0 & \ldots \\
-\rho & 1+\rho^{2} & -\rho & 0 & \ldots \\
0 & -\rho & 1+\rho^{2} & -\rho & \ldots \\
& & \ldots & &
\end{array}\right]
$$

For $\rho$ near 1 this is an approximate Laplacian. $C_{r, c}=\rho^{|r-c|}$ also appears in the literature on the Discrete Cosine Transform (DCT), as a generic covariance matrix for images [198].

2. It is also known in the DCT literature that cosines are the eigenvectors of both $\left[C_{r, c}\right]=\rho^{|r-c|}[198]$ and of the discrete Laplacian approximation (9.12) [225].

\subsubsection{Outlook}

PCA and spline models have complementary advantages and drawbacks. This observation raises the question of whether it is possible to create a unified model that incorporates the relative advantages of each. Such a unified model would be particularly suitable for computer vision tracking applications in which high accuracy is required. In these applications an exact fit to the data is needed (thus necessitating use of a spline), but 
a reasonable shape prior is also beneficial, for example as a robust lowdimensional parameterization of the search space.

We have shown that the data fitting problem for both PCA and LS can be put in the form $\mathbf{c}^{T} \boldsymbol{\Lambda}^{-1} \mathbf{c}+\boldsymbol{\lambda}^{T} \mathbf{S}(\mathbf{U} \mathbf{c}-\mathbf{d})$ with $\boldsymbol{\Lambda}, \mathbf{U}$ obtained from the eigen-decomposition of the covariance and Laplacian operator respectively. The proposed common formulation of PCA and LS suggests a unified model that "inserts" a LS to account for dimensions discarded by the reduced rank PCA, for example by orthogonalizing the LS eigenvectors with respect to the retained PCA basis. The eigenvalues of the combined basis then provide a prior \|\|$_{\Lambda}=\sum c_{k}^{2} / \lambda_{k}$ on the data. Our proposal resembles Probabilistic PCA [233], which inserts an isotropic covariance in the discarded dimensions. It differs in that the LS has a decaying rather than constant spectrum, and prefers smooth deformation.

\subsection{Chapter Summary}

This chapter explored relationships between several techniques that have become commonly used in computer graphics and vision research.

The relationship between RBF and GP regression has been mentioned in passing in several publications, though it does not appear to have been stated in detail, and not all authors are aware of it. For example, the most recent edition of Press et al., Numerical Recipes [196] has adjacent sections on RBF and Gaussian processes, but does not mention a connection. Wahba [241, p. 16 before section 1.5] states an equivalence between GP and Laplacian splines, which implies the RBF equivalence by transitivity (through the fact that the RBF kernel can be seen as the Green's function of the Laplacian spline). Mackay [152] p. 548 says briefly "Generalized radial basis functions ... are closely related to Gaussian processes". Bishop [31] mentions (p. 309) "Thus, if the kernel function ... depends only on the distance ..., then we obtain an expansion in radial basis functions". The second edition of Marsland [155] (2015) added a discussion of Gaussian 
processes and has the clearest statement of this relationship. The presentation in this chapter is based on the author's 2014 conference presentation [132] and slightly predates [155].

The problem of representing faces motivated our exploration of the relationship between splines and PCA. Working in a one-dimensional and regularly sampled setting for simplicity, we conclude that the Laplacian matrix plays a role similar to the inverse of the covariance matrix. Future research (probably by people more talented than this author) may identify a principled way to "insert" the spline in the space discarded by PCA, thereby opening the door to a unified model providing the best of both approaches. The PPCA technique provides a partial precedent for this direction [233].

The most constructive exploration in this chapter considers the effect of taking an eigen-expansion of the RBF kernel and regrouping. This leads to an interesting procedure that can interpolate the results of a PCA representation to arbitrary resolution and in a data-driven way.

The results were demonstrated with examples of data-driven interpolation of motion capture. It is interesting to note that this result is somewhat parallel to the PCA-spline exploration: in both cases the space discarded by PCA is filled with an alternate model. 


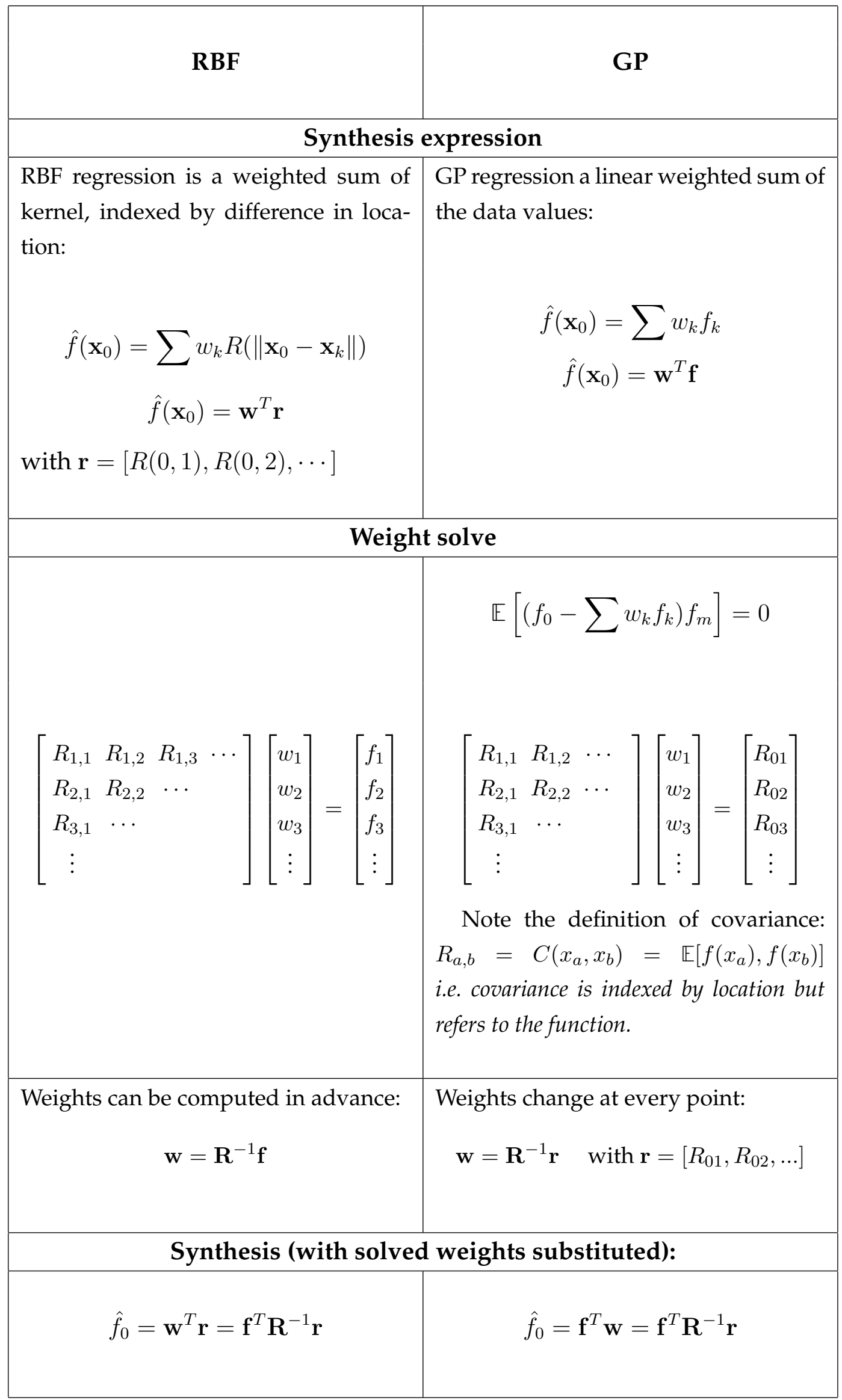

Table 9.1: RBF-GP equivalence. 
206 CHAPTER 9. RELATIONSHIPS BETWEEN VARIOUS MODELS 


\section{Chapter 10}

\section{Conclusions}

Graphics has always borrowed heavily from other fields. The early theory of rendering adopted ideas from projective geometry and signal processing. Quaternions and inverse kinematics came into graphics by way of robotics. Contemporary graphics research applies ideas from increasingly diverse fields.

In general terms, this thesis explores the application of statistical learning ideas to character animation. The outcome has included both new algorithms, and new perspectives on existing methods. I feel the application is obvious, and am certain that the ideas here could have been arrived at sooner by an outsider who is not constrained by a graphics mindset. Nevertheless, this work has had some impact in terms of both academic citations and industry interest. As a single general conclusion, it is safe to say that problems in character animation can sometimes be successfully solved with tools from machine learning, and that such tools are probably more suited to the problem than some other alternatives.

\subsection{Achieved Objectives}

A number of specific research objectives were stated in the introduction. Here we restate these objectives and relate them to the achievements in the 
thesis:

1. To survey statistical learning concepts that are likely to be applicable to the character animation problem. This is done in chapter 2 .

2. To consider facial and body models from a high-dimensional viewpoint. This is done in chapters 6 (for the face case) and 7 (for body deformation).

3. To survey facial animation and body deformation from our examplebased perspective, and show the applicability of associated statistical learning concepts. This is done in chapters 3 and 8 .

4. To illustrate the potential of our new approach by developing several new algorithms for animation. This is done in chapters 4 and 5 .

5. Lastly, to solidify what we have learned by surveying the relationships between several particular techniques. This is done in chapter 9 .

\subsection{Limitations}

From a traditional computer science perspective, the fact that our methods require artistic input could be seen as a limitation. The artists no doubt see things differently. Visual effects and games involve a lot of hand crafted objects, and that is part of their appeal.

A fundamental problem (and one that the author did not appreciate until recently) is the curse of dimensionality and its impact on interpolation and regression. This subject is briefly surveyed in section A.7, and particular instances of the issue are described in Section 3.6.5 and Chapter 6 . As a consequence, we can expect example based methods to work in 10 dimensions, but attempting a 50-dimensional problem will require major compromises. 


\subsection{Future Work}

I believe that we have only scratched the surface of what is possible by applying machine learning to animation problems. For example, I foresee a "directable" data-driven character model, that one can command to walk, run, look older, gain weight, and so on. Strong precedents for such a model already exist, e.g. [147, 118, 12]. This model will appear first in games, where characters must move autonomously.

Perhaps the next frontier will be algorithms for "acting" - enabling characters that can simply be instructed to act drunk, move suspiciously, or act like a cartoon character. There are already precedents in recent research, e.g. [217, 255]. With the recent, dramatic success of deep learning, it is almost possible to imagine a machine that watches every movie and learns to synthesize arbitrary characters.

A core challenge will be to parameterize the resulting capabilities in a human-understandable way. In any case, the general trend will be toward higher-level parameterization and control. Rather than animating individual joint angles, artists will be able to specify what the movement should look like in higher-level terms.

The existing craft of character animation will be increasingly transformed if these developments come into being. On the other hand, others will benefit, since the creation of an animated movie will no longer be something that requires a large budget and team of people. 


\section{Bibliography}

[1] Aggarwal, C. C., Hinneburg, A., and Keim, D. A. On the surprising behavior of distance metrics in high dimensional spaces. In Proceedings of the 8th International Conference on Database Theory (London, UK, UK, 2001), ICDT '01, Springer-Verlag, pp. 420-434.

[2] Alexa, M., Cohen-Or, D., AND Levin, D. As-rigid-as-possible shape interpolation. In Proceedings of the 27th Annual Conference on Computer Graphics and Interactive Techniques (New York, NY, USA, 2000), SIGGRAPH '00, ACM Press / Addison-Wesley Publishing Co., pp. 157-164.

[3] Allen, B., Curless, B., And Popović, Z. The space of human body shapes: Reconstruction and parameterization from range scans. ACM Trans. Graph. 22, 3 (July 2003), 587-594.

[4] Amberg, B., Romdhani, S., And Vetter, T. Optimal step nonrigid icp algorithms for surface registration. In Computer Vision and Pattern Recognition, 2007. CVPR'07. IEEE Conference on (2007), IEEE, pp. 1-8.

[5] Anderson, L., Sosniak, L., AND Bloom, B. Bloom's Taxonomy: A Forty-year Retrospective. National Society for the Study of Education: Yearbook of the National Society for the Study of Education. NSSE, 1994. 
[6] Angelidis, A., Wyvill, G., AND CANI, M. Sweepers: Swept deformation defined by gesture. Graphical Models 68, 1 (2006), 2-14.

[7] Anguelov, D., Srinivasan, P., Koller, D., Thrun, S., RodGERS, J., AND DAVIS, J. Scape: Shape completion and animation of people. ACM Trans. Graph. 24, 3 (July 2005), 408-416.

[8] ANJYO, K., AND LEWIS, J. RBF interpolation and Gaussian process regression through an RKHS formulation. Journal of Mathematics for Industry. 3 (2011), 63-71.

[9] ANJYO, K., AND LEWIS, J. Scattered data interpolation for computer graphics. SIGGRAPH Course, http:/ / portal.acm.org, 2014.

[10] ANJYO, K., AND OCHIAI, H. Mathematical Basics of Motion and Deformation in Computer Graphics. Morgan \& Claypool, 2014.

[11] ANJyo, K., TODO, H., AND LEWIS, J. A practical approach to direct manipulation blendshapes. J. Graphics Tools 16, 3 (2012), 160-176.

[12] ARIKAN, O., AND FORSYTH, D. A. Interactive motion generation from examples. ACM Trans. Graph. 21, 3 (July 2002), 483-490.

[13] Bach, F., Jenatton, R., Mairal, J., And Obozinski, G. Convex optimization with sparsity-inducing norms. In Optimization for Machine Learning, S. Sra, S. Nowozin, and S. Wright, Eds. MIT Press, 2012.

[14] BARAFF, D., AND WitKIN, A. Physically based modeling. ACM SIGGRAPH 2001 Course.

[15] BARAN, I., AND POPOVIĆ, J. Automatic rigging and animation of 3d characters. ACM Trans. Graph. 26, 3 (July 2007).

[16] Barnhill, R., Dube, R., And Little, F. Properties of Shepard's surfaces. Rocky Mountain J. Math. 13 (1983), 365-382. 
[17] Beeler, T., Bickel, B., Beardsley, P., Sumner, B., AND Gross, M. High-quality single-shot capture of facial geometry. ACM Trans. on Graphics (Proc. SIGGRAPH) 29, 3 (2010), 40:1-40:9.

[18] Beeler, T., AND BRAdley, D. Rigid stabilization of facial expressions. ACM Trans. Graph. 33, 4 (July 2014), 44:1-44:9.

[19] Beeler, T., Hahn, F., Bradley, D., Bickel, B., Beardsley, P., Gotsman, C., Sumner, R. W., AND Gross, M. High-quality passive facial performance capture using anchor frames. ACM Trans. Graph. 30 (August 2011), 75:1-75:10.

[20] BeIER, T. personal communication. Hammerhead Productions, 2005.

[21] Beier, T., AND Neely, S. Feature-based image metamorphosis. SIGGRAPH 26, 2 (1992), 35-42.

[22] Bellman, R. Methods of Nonlinear Analysis Vol. 2. Academic Press, 1973.

[23] Bengio, J. C., And Goldenthal, R. Simplicial interpolation for animating The Hulk. In ACM SIGGRAPH 2013 Talks (New York, NY, USA, 2013), SIGGRAPH '13, ACM, pp. 7:1-7:1.

[24] Bergeron, P. 3-d character animation on the symbolics system. SIGGRAPH Course Notes: 3-D Character Animation by Computer (July 1987).

[25] Bermano, A. H., Bradley, D., Beeler, T., Zund, F., NOWrouzezAHraI, D., BARAN, I., SORKINE-HORNung, O., Pfister, H., Sumner, R. W., Bickel, B., AND Gross, M. Facial performance enhancement using dynamic shape space analysis. ACM Trans. Graph. 33, 2 (Apr. 2014), 13:1-13:12. 
[26] Beyer, K. S., GOLDSTEIN, J., RAMAKRISHNAN, R., AND SHAFT, U. When is "nearest neighbor" meaningful? In Proceedings of the 7th International Conference on Database Theory (London, UK, UK, 1999), ICDT '99, Springer-Verlag, pp. 217-235.

[27] Bhat, K. S., Goldenthal, R., YE, Y., MAllet, R., AND KoperWAS, M. High fidelity facial animation capture and retargeting with contours. In Proceedings of the 12th ACM SIGGRAPH/Eurographics Symposium on Computer Animation (New York, NY, USA, 2013), SCA '13, ACM, pp. 7-14.

[28] Bickel, B., Botsch, M., Angst, R., Matusik, W., Otaduy, M., PFISTER, H., AND GROSs, M. Multi-scale capture of facial geometry and motion. ACM Trans. Graph. 26, 3 (2007), 33.

[29] Bickel, B., LANG, M., Botsch, M., Otaduy, M. A., And Gross, M. Pose-space animation and transfer of facial details. In $S C A$ '08: Proceedings of the 2008 ACM SIGGRAPH/Eurographics Symposium on Computer Animation (Aire-la-Ville, Switzerland, Switzerland, 2008), Eurographics Association, pp. 57-66.

[30] BICKEL, P. J., AND LI, B. Local polynomial regression on unknown manifolds. In In Advances in Neural InformationProcessing Systems (NIPS (2006), pp. 2101-2117.

[31] BISHOP, C. Pattern Recognition and Machine Learning. Springer, 2007.

[32] BISHOP, C. M. Neural Networks for Pattern Recognition. Oxford University Press, Inc., New York, NY, USA, 1995.

[33] Blanz, T., AND VetTER, T. A morphable model for the synthesis of 3d faces. In Proceedings of ACM SIGGRAPH (Aug. 1999), ACM SIGGRAPH, pp. 187-194. 
[34] Blanz, V., Basso, C., Poggio, T., and Vetter, T. Reanimating faces in images and video. Computer Graphics Forum(Proceedings of Eurographics 2003) 22, 3 (2003).

[35] Bloomenthal, J. Medial-based vertex deformation. In Proceedings of the 2002 ACM SIGGRAPH/Eurographics Symposium on Computer Animation (New York, NY, USA, 2002), SCA '02, ACM, pp. 147-151.

[36] Bookstein, F. L. Principal warps: Thin-plate splines and the decomposition of deformations. IEEE Trans. Pattern Anal. Mach. Intell. 11 (June 1989), 567-585.

[37] Borshukov, G., Piponi, D., Larsen, O., Lewis, J. P., And TempelaAR-LietZ, C. Universal capture: image-based facial animation for The Matrix Reloaded. In Proceedings of the SIGGRAPH 2003 conference Sketches \& Applications (2003), ACM Press, pp. 1-1.

[38] Borshukov, G., Piponi, D., Larsen, O., Lewis, J. P., AND TEMPELAAR-LiETZ, C. Universal capture: image-based facial animation for "The Matrix Reloaded". In Proceedings of the SIGGRAPH 2003 conference on Sketches \& applications (2003), ACM Press, pp. 1-1.

[39] BotsCh, M., AND SORKINE, O. On linear variational surface deformation methods. IEEE Transactions on Visualization and Computer Graphics 14, 1 (Jan. 2008), 213-230.

[40] Botsch, M., Sumner, R., Pauly, M., and Gross, M. Deformation transfer for detail-preserving surface editing. In Proceedings of Vision, Modeling, and Visualization (VMV) (2006), pp. 357-364.

[41] Bouaziz, S., WANG, Y., AND PAUly, M. Online modeling for realtime facial animation. ACM Trans. Graph. 32, 4 (July 2013), 40:140:10.

[42] Bracewell, R. The Fourier Transform and Its Applications. McGraw Hill, 2000. 
[43] Bradley, D., Heidrich, W., Popa, T., And Sheffer, A. High resolution passive facial performance capture. ACM Trans. Graph. 29, 4 (July 2010), 41:1-41:10.

[44] BROOMHEAD, D., AND LOWE, D. Multivariable functional interpolation and adaptive networks. Complex Systems 2 (1988), 321-355.

[45] Buhmann, M. D. Radial Basis Functions : Theory and Implementations. Cambridge University Press, 2003.

[46] Busch, J. Facing second son. fxguide.com http:/ / www.fxguide.com/featured/facing-second-son.

[47] CAFlisCH, R. Monte carlo and quasi-monte carlo methods, 1998.

[48] CAO, C., HOU, Q., AND ZHOU, K. Displaced dynamic expression regression for real-time facial tracking and animation. ACM Trans. Graph. 33, 4 (July 2014), 43:1-43:10.

[49] CAO, C., WenG, Y., LIN, S., AND ZHOU, K. 3d shape regression for real-time facial animation. ACM Trans. Graph. 32, 4 (July 2013), 41:1-41:10.

[50] CaO, Y., Faloutsos, P., AND Pighin, F. Unsupervised learning for speech motion editing. In Proceedings of the 2003 ACM SIGGRAPH/Eurographics symposium on Computer animation (Aire-laVille, Switzerland, Switzerland, 2003), SCA '03, Eurographics Association, pp. 225-231.

[51] Carr, J. C., Beatson, R. K., Cherrie, J. B., Mitchell, T. J., Fright, W. R., MCCAllum, B. C., AND Evans, T. R. Reconstruction and representation of $3 \mathrm{~d}$ objects with radial basis functions. In SIGGRAPH '01: Proceedings of the 28th annual conference on Computer graphics and interactive techniques (New York, NY, USA, 2001), ACM, pp. 67-76. 
[52] Casciola, G., Montefusco, L., AND Morigi, S. Edge-driven image interpolation using adaptive anisotropic radial basis functions. Journal of Mathematical Imaging and Vision 36, 2 (2010), 125-139.

[53] Cetinaslan, O., Orvalho, V., And Lewis, J. Sketch-based controllers for blendshape facial animation. In Eurographics (2015).

[54] Chai, J., ANd Hodgins, J. K. Performance animation from lowdimensional control signals. In SIGGRAPH '05: ACM SIGGRAPH 2005 Papers (New York, NY, USA, 2005), ACM, pp. 686-696.

[55] Chai, J.-X., XIAO, J., AND Hodgins, J. Vision-based control of $3 \mathrm{~d}$ facial animation. In Proceedings of the 2003 ACM SIGGRAPH/Eurographics symposium on Computer animation (Aire-laVille, Switzerland, Switzerland, 2003), SCA '03, Eurographics Association, pp. 193-206.

[56] Chen, D. T., ANd Zeltzer, D. Pump it up: computer animation of a biomechanically based model of muscle using the finite element method. SIGGRAPH Comput. Graph. 26, 2 (July 1992), 89-98.

[57] Chen, F., AND Suter, D. Multiple order laplacian splines - including splines with tension. Technical report, Monash University, Clayton 3168, Australia, 1996.

[58] ChenG, Z.-Q., WANG, Y.-Z., B. LI, K. X., DANG, G., AND S. Y.-Jin. A survey of methods for moving least squares surfaces. In IEEE/EG Symposium on Volume and Point-Based Graphics (2008).

[59] Cherkassky, V. S., AND Mulier, F. Learning from Data: Concepts, Theory, and Methods, 1st ed. John Wiley \& Sons, Inc., New York, NY, USA, 1998.

[60] CHOE, B., AND Ko, H.-S. Analysis and synthesis of facial expressions with hand-generated muscle actuation basis. In Computer Animation and Social Agents (CASA) (2001). 
[61] Choe, B., LeE, H., AND Ko, H.-S. Performance-driven musclebased facial animation. J. Visualization and Computer Animation 12, 2 (2001), 67-79.

[62] Choe, B., LeE, H., AND Ko, H.-S. Performance-driven musclebased facial animation. In Proceedings of Computer Animation (May 2001), vol. 12, pp. 67-79.

[63] Chuang, E., AND Bregler, C. Performance driven facial animation using blendshape interpolation. CS-TR-2002-02, Department of Computer Science, Stanford University (2002).

[64] Chuang, E., Deshrande, H., ANd Bregler, C. Facial expression space learning. In Proceedings of Pacific Graphics (2002).

[65] Chui, H., AND RANGARAJAN, A. A new point matching algorithm for non-rigid registration. Computer Vision and Image Understanding 89 , 2-3 (2003), $114-141$.

[66] Clutterbuck, S., AND Jacobs, J. A physically based approach to virtual character deformations. ACM SIGGRAPH Talk, session Avatar in Depth, 2010.

[67] CMU Graphics Lab Motion Capture Database. http:/ / mocap.cs.cmu.edu, 2015.

[68] Comaniciu, D., AND MeER, P. Mean shift: A robust approach toward feature space analysis. IEEE Trans. Pattern Anal. Mach. Intell. 24,5 (2002), 603-619.

[69] Cootes, T. F., Edwards, G. J., And Taylor, C. J. Active Appearance Models, vol. 1407 of Lecture Notes in Computer Science. Springer, 1998. 
[70] Dale, K., Sunkavalli, K., Johnson, M. K., Vlasic, D., MaTUSIK, W., AND PFISTER, H. Video face replacement. ACM Trans. Graph. 30, 6 (Dec. 2011), 130:1-130:10.

[71] Decarlo, D., And Metaxas, D. The integration of optical flow and deformable models with applications to human face shape and motion estimation. In Proceedings, Conference on Computer Vision and Pattern Recognition (1996), pp. 231-238.

[72] Deng, Z., Bailenson, J., Lewis, J. P., And Neumann, U. Perceiving visual emotions with speech. In Proc. of the 6th Int'l Conf. on Intelligent Virtual Agents (IVA) 2006 (August 2006), pp. 107-120.

[73] Deng, Z., Chiang, P. Y., Fox, P., And Neumann, U. Animating blendshape faces by cross-mapping motion capture data. In Proc. of ACM SIGGRAPH Symposium on Interactive 3D Graphics and Games (I3D) (Mar. 2006), pp. 43-48.

[74] Deng, Z., AND NoH, J. Computer facial animation: A survey. In Data-Driven 3D Facial Animation (2007), Springer, pp. 1-28.

[75] Ding, C., AND YE, J. Two-dimensional singular value decomposition (2dsvd) for 2d maps and images. In Proc. SIAM Int'l Conf. Data Mining (SDM'05) (April 2005), pp. 32-43.

[76] Dinh, H. Q., TURK, G., AND Slabaugh, G. Reconstructing surfaces using anisotropic basis functions. In Computer Vision, 2001. ICCV 2001. Proceedings. Eighth IEEE International Conference on (2001), vol. 2, IEEE, pp. 606-613.

[77] Donato, G., And Belongie, S. Approximate thin plate spline mappings. In Proceedings of the 7th European Conference on Computer Vision-Part III (London, UK, UK, 2002), ECCV '02, Springer-Verlag, pp. 21-31. 
[78] Dow, M. Explicit inverses of Toeplitz and associated matrices. ANZIAM J. 44 E (2003), E185-E215.

[79] Duchon, J. Math. comp. Interpolation des Fonctions de Deux Variables Suivant le Principe de la Flexion des Plaques Minces 10 (1976), 5-12.

[80] EKMAN, P. Duchenne and facial expression of emotion. In The mechanism of human facial expression, edited and translated by $R$. Andrew Cuthbertson. Cambridge, 1990.

[81] Ekman, P., And Rosenberg, E. What the Face Reveals: Basic and Applied Studies of Spontaneous Expression Using the Facial Action Coding System (FACS). Series in affective science. Oxford University Press, 1997.

[82] ElaD, M. Sparse and Redundant Representations: From Theory to Applications in Signal and Image Processing, 1st ed. Springer Publishing Company, Incorporated, 2010.

[83] ELSON, M. "Displacement" facial animation techniques. SIGGRAPH Course Notes: State of the Art in Facial Animation (1990).

[84] EYETRONICS. Shapesnatcher. http://www.eyetronics.com.

[85] Fasshauer, G. F. Meshfree Approximation Methods with MATLAB. World Scientific Publishing Co., Inc., River Edge, NJ, USA, 2007.

[86] Fieguth, P. Statistical Image Processing and Multidimensional Modeling. Information science and statistics. Springer, New York, 2011.

[87] FlueCKiger, B. Computer-generated characters in Avatar and Benjamin Button. In: H. Segeberg (ed.) Digitalitat und Kino. Translation from German by B. Letzler, 2011.

[88] Fordham, J. Middle earth strikes back. Cinefex, 92 (2003), 71-142. 
[89] FORSEY, D. A surface model for skeleton-based character animation. In Second Eurographics Workshop on Animation and Simulation (1991), pp. 55-73.

[90] FXGUIDE.COM. fxpodcast: Dr. Mark Sagar, 2011.

[91] Fyffe, G., Hawkins, T., Watts, C., MA, W., And Debevec, P. E. Comprehensive facial performance capture. Comput. Graph. Forum 30,2 (2011), 425-434.

[92] GARCKE, J. Sparse grid tutorial. http://www.math.tu-berlin.de/ garcke/paper/sparseGridTutorial.pdf.

[93] Garrido, P., Valgaerts, L., Wu, C., And Theobalt, C. Reconstructing detailed dynamic face geometry from monocular video. ACM Trans. Graphics (Proc. SIGGRAPH Asia) 32, 6 (2013).

[94] Gentle, J. Random Number Generation and Monte Carlo Methods. Statistics and Computing. Springer, 2003.

[95] Gleicher, M., AND WiTKIN, A. Through-the-lens camera control. SIGGRAPH 26, 2 (July 1992), 331-340.

[96] Green, P. J., And Silverman, B. W. Nonparametric Regression and Generalized Linear Models: A Roughness Penalty Approach. Chapman \& Hall, London, 1994.

[97] Grinspun, E., Desbrun, M., AND ET AL. Discrete differential geometry: An applied introduction. SIGGRAPH Course, 2006.

[98] GU, C. Smoothing Spline ANOVA Models, 2nd Ed. Springer, 2013.

[99] Guenter, B., Grimm, C., Wood, D., Malvar, H., And Pighin, F. Making faces. In SIGGRAPH 98 Conference Proceedings (July 1998), ACM SIGGRAPH, pp. 55-66. 
[100] Hastie, T., Tibshirani, R., And Friedman, J. The Elements of Statistical Learning: Data Mining, Inference and Prediction. Springer Verlag, New York, NY, 2009.

[101] Havaldar, P. Monster House. In SIGGRAPH'06: ACM SIGGRAPH 2006 Courses (2006).

[102] Henne, M., AND Hickel, H. The making of "toy story". In Proceedings of the 41st IEEE International Computer Conference (Washington, DC, USA, 1996), COMPCON '96, IEEE Computer Society, pp. 463-.

[103] Hsu, W. M., Hughes, J. F., And Kaufman, H. Direct manipulation of free-form deformations. In Proceedings of the 19th Annual Conference on Computer Graphics and Interactive Techniques (New York, NY, USA, 1992), SIGGRAPH '92, ACM, pp. 177-184.

[104] Huber, P. J. Robust Statistical Procedures, 2nd ed. SIAM, Philadelphia, 1977.

[105] HwAnG, B.-W., AND LEE, S.-W. Reconstruction of partially damaged face images based on a morphable face model. IEEE Transactions on Pattern Analysis and Machine Intelligence 25, 3 (2003), 365-372.

[106] Hyneman, W., ItokazU, H., Williams, L., and Zhao, X. Human face project. In ACM SIGGRAPH 2005 Courses (New York, NY, USA, 2005), SIGGRAPH '05, ACM.

[107] Jacobson, A., Deng, Z., Kavan, L., AND LeWIS, J. Skinning: Real-time shape deformation. SIGGRAPH Course, http:/ /skinning.org, 2014.

[108] JONES, S. Digital Creature Rigging: The Art and Science of CG Creature Setup in 3ds Max. Taylor \& Francis, 2012. 
[109] Joshi, P., Meyer, M., DeRose, T., Green, B., And Sanocki, T. Harmonic coordinates for character articulation. ACM Trans. Graph. 26, 3 (July 2007).

[110] Joshi, P., Tien, W. C., Desbrun, M., And Pighin, F. Learning controls for blend shape based realistic facial animation. Eurographics/SIGGRAPH Symposium on Computer Animation (SCA) (2003).

[111] Kähler, K., Haber, J., Yamauchi, H., AND Seidel, H.-P. Head shop: Generating animated head models with anatomical structure. In Proceedings of the 2002 ACM SIGGRAPH/Eurographics Symposium on Computer Animation (New York, NY, USA, 2002), SCA '02, ACM, pp. 55-63.

[112] KaUfman, D. Vfx crossroads: Causes and effects of an industry crisis. creativecow.net https:/ /library.creativecow.net/kaufman_debra/VFX_Crossroads1/1, 2013.

[113] Kavan, L., Collins, S., ŽÁra, J., AND O'Sullivan, C. Skinning with dual quaternions. In Proceedings of the 2007 Symposium on Interactive 3D Graphics and Games (New York, NY, USA, 2007), I3D '07, ACM, pp. 39-46.

[114] Kim, P. H., SEOL, Y., SONG, J., AND NOH, J. Facial Retargeting by Adding Supplemental Blendshapes. In Pacific Graphics (2011), pp. 89-92.

[115] KLEISER, J. A fast, efficient, accurate way to represent the human face. In SIGGRAPH '89 Course Notes 22: State of the Art in Facial Animation (1989).

[116] KOMATSU, K. Human skin model capable of natural shape variation. The Visual Computer 3, 5 (1988), 265-271. 
[117] Komorowski, D., Melapudi, V., Mortillaro, D., And LeE, G. S. A hybrid approach to facial rigging. In ACM SIGGRAPH ASIA 2010 Sketches (New York, NY, USA, 2010), SA '10, ACM, pp. 42:142:2.

[118] Kovar, L., Gleicher, M., And Pighin, F. Motion graphs. In Proceedings of the 29th Annual Conference on Computer Graphics and Interactive Techniques (New York, NY, USA, 2002), SIGGRAPH '02, ACM, pp. 473-482.

[119] KRY, P. G., JAMES, D. L., AND PAI, D. K. EigenSkin: Real time large deformation character skinning in hardware. In Proceedings of the 2002 ACM SIGGRAPH Symposium on Computer Animation (SCA-02) (New York, Jul. 2002), S. N. Spencer, Ed., ACM Press, pp. 153-160.

[120] Kurihara, T., AND MiyatA, N. Modeling deformable human hands from medical images. In Proceedings of the 2004 ACM SIGGRAPH Symposium on Computer Animation (SCA-04) (2004), pp. 357366.

[121] LANCASTER, P., AND SALKAUSKAS, K. Curve and surface fitting: an introduction. Computational mathematics and applications. Academic Press, 1986.

[122] LAU, M., CHAI, J., XU, Y.-Q., AND SHUM, H.-Y. Face poser: Interactive modeling of $3 \mathrm{~d}$ facial expressions using facial priors. ACM Trans. Graph. 29, 1 (2009), 1-17.

[123] Lay, B., Sparrow, W., Hughes, K., AND O’Dwyer, N. Practice effects on coordination and control, metabolic energy expenditure, and muscle activation. Human movement science 21, 5 (2002), 807-830.

[124] LeE, A., DobKIN, D., Sweldens, W., AND SchrÖDER, P. Multiresolution mesh morphing. In Proceedings of SIGGRAPH 99 (August 1999), pp. 343-350. 
[125] LeE, D., AND SeUnG, H. S. Learning the parts of objects by nonnegative matrix factorization. Nature, 401 (1999), 788-791.

[126] LEE, G. S. Evaluation of the radial basis function space. In ACM SIGGRAPH ASIA 2009 Sketches (New York, NY, USA, 2009), SIGGRAPH ASIA '09, ACM, pp. 42:1-42:1.

[127] LeE, G. S., AND HANNER, F. Practical experiences with pose space deformation. In ACM SIGGRAPH ASIA 2009 Sketches (New York, NY, USA, 2009), SIGGRAPH ASIA '09, ACM, p. 43.

[128] LeVI, Z., AND LEVIN, D. Shape deformation via interior RBF. IEEE Trans. Vis. Comput. Graph. 20, 7 (2014), 1062-1075.

[129] LEWIS, J. Lifting detail from darkness. In SIGGRAPH '01: Proceedings of the SIGGRAPH 2001 conference Sketches \& applications (2001), ACM Press.

[130] LEWIS, J., AND ANJYO, K. Identifying salient points. In SIGGRAPH Asia short papers (2009).

[131] Lewis, J., And Anjyo, K. Direct manipulation blendshapes. Computer Graphics and Applications (special issue: Digital Human Faces) 30, 4 (2010), 42-50.

[132] LEWIS, J., ANJYO, K., AND RHEE, T. Superresolution from principal component models by RKHS sampling. In Mathematical Progress in Expressive Image Synthesis II (2015), Springer.

[133] Lewis, J., Hwang, H.-J., NeumanN, U., And Enciso, R. Smart point landmark distribution for thin-plate splines. In Proc. SPIE Medical Imaging (San Diego, 2004), pp. 1236-1243.

[134] Lewis, J., Mo, Z., , AND NeumanN, U. Ripple-free local bases by design. In Proc. Int. Conf. on Acoustics, Speech and Signal Processing (ICASSP) (2004), pp. 684-688. 
[135] Lewis, J., Mo, Z., ANJYo, K., AND Rhee, T. Probable and improbable faces. In Mathematical Progress in Expressive Image Synthesis I. Springer, 2014.

[136] Lewis, J., Mooser, J., DenG, Z., And Neumann, U. Reducing blendshape interference by selected motion attenuation. In SI3D '05: Proceedings of the 2005 symposium on Interactive 3D graphics and games (New York, NY, USA, 2005), ACM Press, pp. 25-29.

[137] Lewis, J., Pighin, F., AND ANJYO, K. Scattered data interpolation for computer graphics. SIGGRAPH Asia Course, http://portal.acm.org, 2010.

[138] LEWIS, J. P. Generalized stochastic subdivision. ACM Transactions on Graphics 6, 3 (July 1987), 167-190.

[139] LeWis, J. P., Cordner, M., AND FonG, N. Pose space deformation: a unified approach to shape interpolation and skeletondriven deformation. In SIGGRAPH '00: Proceedings of the 27th annual conference on Computer graphics and interactive techniques (New York, NY, USA, 2000), ACM Press/Addison-Wesley Publishing Co., pp. $165-172$.

[140] Li, D., Sueda, S., NeOG, D. R., AND PAI, D. K. Thin skin elastodynamics. ACM Trans. Graph. (Proc. SIGGRAPH) 32, 4 (July 2013), 49:1-49:9.

[141] LI, H., Weise, T., AND PAuly, M. Example-based facial rigging. ACM Transactions on Graphics (Proceedings SIGGRAPH 2010) 29, 3 (July 2010).

[142] LI, H., YU, J., YE, Y., AND BREGLER, C. Realtime facial animation with on-the-fly correctives. ACM Transactions on Graphics 32, 4 (July 2013), 42:1-42:10. 
[143] LI, M., AND VitÁNYI, P. An Introduction to Kolmogorov Complexity and its Applications. Springer Verlag, New York, 1997.

[144] LI, Q., AND DENG, Z. Orthogonal blendshape based editing system for facial motion capture data. IEEE Computer Graphics and Applications (CGEA) 28, 6 (Nov. 2008), 76-82.

[145] LI, Y., AND ITO, W. Shape parameter optimization for Adaboosted active shape model. ICCV 1 (2005), 251-258.

[146] Lipman, Y., Levin, D., AND Cohen-OR, D. Green coordinates. ACM Trans. Graph. 27, 3 (Aug. 2008), 78:1-78:10.

[147] Loper, M., Mahmood, N., AND Black, M. J. Mosh: Motion and shape capture from sparse markers. ACM Trans. Graph. 33, 6 (Nov. 2014), 220:1-220:13.

[148] MA, W.-C., BARBATI, M., AND LeWIS, J. P. A facial composite editor for blendshape characters. In Proceedings of the Digital Production Symposium (New York, NY, USA, 2012), DigiPro '12, ACM, pp. 2126.

[149] MA, W.-C., JONES, A., ChiAnG, J.-Y., HAWKins, T., FrederikSen, S., Peers, P., Vukovic, M., Ouhyoung, M., And Debevec, $P$. Facial performance synthesis using deformation-driven polynomial displacement maps. ACM Trans. Graph. 27, 5 (Dec. 2008), 121:1121:10.

[150] MA, W.-C., WANG, Y.-H., FYFFe, G., Chen, B.-Y., AND DebeveC, P. A blendshape model that incorporates physical interaction. Computer Animation and Virtual Worlds 23, 3-4 (2012), 235-243.

[151] MACKAY, D. J. Hyperparameters: Optimize, or integrate out? In Maximum entropy and Bayesian methods. Springer, 1996, pp. 43-59. 
[152] MACKAY, D. J. C. Information Theory, Inference E Learning Algorithms. Cambridge University Press, New York, NY, USA, 2002.

[153] Magnenat-Thalmann, N., LAperriere, R., And Thalmann, D. Joint-dependent local deformations for hand animation and object grasping. In Proc. Graphics Interface (1988), pp. 26-33.

[154] Magnenat-Thalmann, N., And Thalmann, D. Human body deformations using joint-dependent local operators and finiteelement theory. In Making Them Move, N. I. Badler, B. A. Barsky, and D. Zeltzer, Eds. Morgan Kaufmann Publishers Inc., San Francisco, CA, USA, 1991, pp. 243-262.

[155] Marsland, S. Machine Learning: An Algorithmic Perspective, 2nd ed. Chapman \& Hall/CRC, 2015.

[156] Matthews, I., XiaO, J., AND BAKer, S. On the dimensionality of deformable face models. CMU-RI-TR-06-12, 2006.

[157] MClaughlin, T., Cutler, L., And Coleman, D. Character rigging, deformations, and simulations in film and game production. In ACM SIGGRAPH 2011 Courses (New York, NY, USA, 2011), SIGGRAPH '11, ACM, pp. 5:1-5:18.

[158] Merry, B., Marais, P., AND Gain, J. Animation space: A truly linear framework for character animation. ACM Trans. Graph. 25, 4 (2006), 1400-1423.

[159] MeYer, M., AND ANDERSON, J. Key point subspace acceleration and soft caching. ACM Trans. Graph. 26, 3 (2007), 74.

[160] Meytlis, M., AND Sirovich, L. On the dimensionality of face space. IEEE Trans. Pattern Anal. Mach. Intell. 29, 7 (2007), 1262-1267. 
[161] Miranda, J. C., Alvarez, X., Orvalho, J. A., Gutierrez, D., SOUSA, A. A., AND ORVALHO, V. Sketch express: Facial expressions made easy. In Proceedings of the Eighth Eurographics Symposium on Sketch-Based Interfaces and Modeling (New York, NY, USA, 2011), SBIM '11, ACM, pp. 87-94.

[162] Mo, Z., LEWIS, J., AND NeUMANN, U. Face inpainting with local linear representations. In BMVC (2004), BMVA, pp. 347-356.

[163] MoHR, A., AND GLeICHER, M. Building efficient, accurate character skins from examples. ACM Transactions on Graphics 22, 3 (July 2003), 562-568.

[164] MoOdY, J., AND DARKEN, C. Learning with localized receptive fields. In Proceedings of the 1988 Connectionist Models Summer School, D. S. Touretzky, G. E. Hinton, and T. J. Sejnowski, Eds. San Francisco, CA: Morgan Kaufmann, 1989, pp. 133-143.

[165] MovA LLC. Contour reality capture. http://www.mova.com (2009).

[166] Murtagh, D. Pose-space deformation on top of dual quaternion skinning. M.S. Thesis, U. Dublin, 2008.

[167] Nealen, A., Mller, M., Keiser, R., Boxerman, E., AND CarlSON, M. Physically Based Deformable Models in Computer Graphics. In Eurographics 2005 - State of the Art Reports (2005), Y. Chrysanthou and M. Magnor, Eds., The Eurographics Association.

[168] Nedel, L., AND Thalmann, D. Modeling and deformation of the human body using an anatomically-based approach. www preprint.

[169] Neumann, T., Varanasi, K., Wenger, S., Wacker, M., MaGNOR, M., AND THEOBALT, C. Sparse localized deformation components. ACM Transactions on Graphics (Proceedings SIGGRAPH Asia) 32, 6 (2013). 
[170] NOH, J., AND NEUMANN, U. A survey of facial modeling and animation techniques. In USC Technical Report 99-705 (1999), U. Southern California.

[171] NOH, J., AND NeUmAnN, U. Expression cloning. In SIGGRAPH 2001, Computer Graphics Proceedings (2001), E. Fiume, Ed., ACM Press / ACM SIGGRAPH, pp. 277-288.

[172] Olshausen, B. A., AND Field, D. J. Emergence of simple-cell receptive field properties by learning a sparse code for natural images. Nature 13 June, 381 (1996), 607-609.

[173] Orvalho, V., Bastos, P., Parke, F., Oliveira, B., , ANd AlVAREZ, X. A facial rigging survey: State of the art report. In Eurographics (2012), pp. 183-204.

[174] OsIPA, J. Stop Staring: Facial Modeling and Animation Done Right, 2nd Ed. Sybex, 2007.

[175] Öztireli, A. C., BAran, I., PopA, T., DAlstein, B., SuMNER, R. W., AND GROSS, M. Differential blending for expressive sketch-based posing. In Proceedings of the 2013 ACM SIGGRAPH/Eurographics Symposium on Computer Animation (New York, NY, USA, 2013), SCA '13, ACM.

[176] Papoulis, A. Probability, Random Variables, and Stochastic Processes. McGraw Hill, New York, 1975.

[177] PARKE, F. personal communication. Texas A\&M University.

[178] PARKe, F. Computer generated animation of faces. Proceedings ACM annual conference. (Aug. 1972).

[179] PARKE, F. A parametric model for human faces. PhD thesis, University of Utah, Salt Lake City, Utah, Dec. 1974. UTEC-CSc-75-047. 
[180] PARKE, F. Control parametrization for facial animation. In Computer Animation 91, N. M. Thalmann and D. Thalmann, Eds. SpringerVerlag, Tokyo, 1991, pp. 3-14.

[181] PARKe, F. I. Parameterized models for facial animation. IEEE Computer Graphics and Applications 2, 9 (1992), 61-68.

[182] PARKe, F. I., AND WATERS, K. Computer Facial Animation. AK Peters Ltd, 2008.

[183] PATEL, A., AND SMITH, W. A. P. 3D morphable face models revisited. In Conference on Computer Vision and Pattern Recognition (CVPR) (Los Alamitos, CA, USA, 2009), IEEE Computer Society, pp. 13271334.

[184] Penev, P. S., And Sirovich, L. The global dimensionality of face space. In Proc. 4th Int'l Conf. Automatic Face and. Gesture Recognition (2000), pp. 264-270.

[185] Pérez, P., Gangnet, M., And Blake, A. Poisson image editing. ACM Trans. Graph. 22, 3 (2003), 313-318.

[186] Phillips, P. J., Wechsler, H., Huang, J., And Rauss, P. The feret database and evaluation procedure for face recognition algorithms. Image and Vision Computing J. 16, 5 (1998), 295-306.

[187] PIEPer, S. Physically-based animation of facial tissue for surgical simulation. SIGGRAPH 89 Tutorial Notes: State of the Art in Facial Animation (1989).

[188] Pighin, F., Hecker, J., Lischinski, D., Szeliski, R., AND SALESIN, D. Synthesizing realistic facial expressions from photographs. In SIGGRAPH 98 Conference Proceedings (July 1998), ACM SIGGRAPH, pp. 75-84. 
[189] PIgHIN, F., AND LEWIS, J. Digital face cloning. SIGGRAPH Course, http://portal.acm.org, 2005.

[190] Pighin, F., AND LEWIS, J. Performance-driven facial animation. SIGGRAPH Course, http:/ / portal.acm.org, 2006.

[191] Pighin, F., AND LEWIS, J. Practical least squares for computer graphics. SIGGRAPH Course, http:/ / portal.acm.org, 2007.

[192] Pighin, F., Szeliski, R., AND SAlesin, D. Resynthesizing facial animation through $3 \mathrm{~d}$ model-based tracking. In Proceedings, International Conference on Computer Vision (1999).

[193] Pourahmadi, M. High-Dimensional Covariance Estimation: With High-Dimensional Data. Wiley Series in Probability and Statistics. Wiley, 2013.

[194] POWELL, J. D. The theory of radial basis function approximation. Cambridge University Numerical Analysis Report, 1990.

[195] POWELL, M. J. D. Radial basis functions for multivariable interpolation: A review. In Algorithms for Approximation, J. C. Mason and M. G. Cox, Eds. Clarendon Press, New York, NY, USA, 1987, pp. 143167.

[196] Press, W. H., Teukolsky, S. A., Vetterling, W. T., And FlanNERY, B. P. Numerical Recipes 3rd Edition: The Art of Scientific Computing, 3 ed. Cambridge University Press, New York, NY, USA, 2007.

[197] RAITT, B. The making of Gollum. Presentation at U. Southern California Institute for Creative Technologies's Frontiers of Facial Animation Workshop, August 2004.

[198] RaO, K., AND YIP, P. Discrete Cosine Transform: Algorithms, Advantages, Applications. Academic Press, 1990. 
[199] Rasmussen, C., AND Williams, C. Gaussian Processes for Machine Learning. Adaptive Computation And Machine Learning. MIT Press, 2006.

[200] Rhee, T., Hwang, Y., Kim, J. D., AND Kim, C. Real-time facial animation from live video tracking. In Proceedings of the 2011 ACM SIGGRAPH/Eurographics Symposium on Computer Animation (New York, NY, USA, 2011), SCA '11, ACM, pp. 215-224.

[201] Rhee, T., LeWis, J., Neumann, U., And Nayak, K. Scan-based volume animation driven by locally adaptive articulated registrations. IEEE Trans. Visualization and Computer Graphics (2011).

[202] Roweis, S. T., AND SAUL, L. K. Nonlinear dimensionality reduction by locally linear embedding. SCIENCE 290 (2000), 2323-2326.

[203] Russell, J. A. A circumplex model of affect. J. Personality and Social Psychology 39 (1980), 1161-1178.

[204] SAgar, M., AND GROSSMAN, R. Facial performance capture and expressive translation for King Kong. In SIGGRAPH 2006 Sketches (2006).

[205] SAITO, J. Smooth contact-aware facial blendshapes transfer. In Proceedings of the Symposium on Digital Production (New York, NY, USA, 2013), DigiPro '13, ACM, pp. 7-12.

[206] SalzmanN, M., AND URTASUn, R. Implicitly constrained Gaussian process regression for monocular non-rigid pose estimation. In Advances in Neural Information Processing Systems 23, J. Lafferty, C. Williams, J. Shawe-Taylor, R. Zemel, and A. Culotta, Eds. Curran Associates, Inc., 2010, pp. 2065-2073.

[207] Scheepers, F., Parent, R. E., Carlson, W. E., and May, S. F. Anatomy-based modeling of the human musculature. In Proceed- 
ings of the 24th Annual Conference on Computer Graphics and Interactive Techniques (New York, NY, USA, 1997), SIGGRAPH '97, ACM Press/Addison-Wesley Publishing Co., pp. 163-172.

[208] SCHNEIDER, D., AND EISERT, P. Fast nonrigid mesh registration with a data-driven deformation prior. In Computer Vision Workshops (ICCV Workshops), 2009 IEEE 12th International Conference on (Sept 2009), pp. 304-311.

[209] Scholkopf, B., AND SmolA, A. Learning with Kernels. MIT Press, 2001.

[210] Scotт, D. W. Multivariate Density Estimation. Wiley and Sons, 1992.

[211] SederberG, T. W., AND PARry, S. R. Free-form deformation of solid geometric models. SIGGRAPH Comput. Graph. 20, 4 (Aug. 1986), 151-160.

[212] SeO, J., Irving, G., Lewis, J. P., AND NOH, J. Compression and direct manipulation of complex blendshape models. ACM Trans. Graph. 30, 6 (Dec. 2011), 164:1-164:10.

[213] SEOL, Y., AND LEWIS, J. Tuning facial animation in a mocap pipeline. ACM SIGGRAPH Talk, 2014.

[214] SeOl, Y., LeWIS, J., SeO, J., Choi, B., Anjyo, K., AND NoH, J. Spacetime expression cloning for blendshapes. ACM Trans. Graph. 31, 2 (Apr. 2012), 14:1-14:12.

[215] SEOL, Y., SEO, J., KIM, P. H., LEWIS, J., AND NOH, J. Artist friendly facial animation retargeting. ACM Transactions on Graphics (TOG) 30, 6 (2011), 162.

[216] SeOL, Y., SEO, J., KiM, P. H., LEWIS, J. P., AND NOH, J. Weighted pose space editing for facial animation. The Visual Computer 28, 3 (2012), 319-327. 
[217] Shapiro, A., CaO, Y., AND Faloutsos, P. Style components. In GI '06: Proceedings of Graphics Interface 2006 (Toronto, Ont., Canada, Canada, 2006), Canadian Information Processing Society, pp. 33-39.

[218] SIBSON, R. A brief description of natural neighbor interpolation. In Interpreting Multivariate Data, V. Barnett, Ed. John Wiley, Chichester, 1981, chapter 2, pp. 21-36.

[219] Sifakis, E., Neverov, I., AND FedkiW, R. Automatic determination of facial muscle activations from sparse motion capture marker data. ACM Trans. Graph. 24, 3 (July 2005), 417-425.

[220] Singer, G. The Two Towers: Face to face with Gollum. Animation World Network, March 2003.

[221] Singh, K., AND Fiume, E. Wires: A geometric deformation technique. In Proceedings of the 25th Annual Conference on Computer Graph$i c s$ and Interactive Techniques (New York, NY, USA, 1998), SIGGRAPH '98, ACM, pp. 405-414.

[222] Sloan, P.-P. J., Rose, C. F., And Cohen, M. F. Shape by example. In SI3D '01: Proceedings of the 2001 symposium on Interactive 3D graphics (New York, NY, USA, 2001), ACM Press, pp. 135-143.

[223] Sorkine, O., AND AlexA, M. As-rigid-as-possible surface modeling. In Proceedings of the Fifth Eurographics Symposium on Geometry Processing (Aire-la-Ville, Switzerland, Switzerland, 2007), SGP '07, Eurographics Association, pp. 109-116.

[224] Sparrow, W. Energetics of Human Activity. Human Kinetics, 2000.

[225] Strang, G. The discrete cosine transform. SIAM Rev. 41, 1 (Mar. 1999), 135-147.

[226] Sumner, R. W., AND Popović, J. Deformation transfer for triangle meshes. ACM Trans. Graph. 23, 3 (Aug. 2004), 399-405. 
[227] Sumner, R. W., Zwicker, M., Gotsman, C., And Popović, J. Mesh-based inverse kinematics. ACM Trans. Graph. 24, 3 (July 2005), 488-495.

[228] TAYLOR, P. personal communication. Disney/Dream Quest Mighty Joe Young facial animation.

[229] Tena, J. R., De la Torre, F., and Matthews, I. Interactive region-based linear 3d face models. In ACM SIGGRAPH 2011 papers (New York, NY, USA, 2011), SIGGRAPH '11, ACM, pp. 76:1-76:10.

[230] Tenenbaum, J. B., De Silva, V., And Langford, J. C. A global geometric framework for nonlinear dimensionality reduction. Science 290 (2000), 2319-2323.

[231] Terzopoulos, D., And Waters, K. Physically-based facial modeling, analysis, and animation. Journal of Visualization and Computer Animation 2, 4 (Oct. 1991), 73-80.

[232] Tickoo, S. Autodesk Maya 2010: A Comprehensive Guide. CADCIM Technologies, 2009.

[233] Tipping, M. E., AND Bishop, C. M. Probabilistic principal component analysis. Journal of the Royal Statistical Society, Series B 61 (1999), 611-622.

[234] TURK, G., AND O'BRIEN, J. F. Shape transformation using variational implicit functions. In Proceedings of the 26th Annual Conference on Computer Graphics and Interactive Techniques (New York, NY, USA, 1999), SIGGRAPH '99, ACM Press / Addison-Wesley Publishing Co., pp. 335-342.

[235] TuRner, R., AND Thalmann, D. The elastic surface layer model for animated character construction. In Proc. Computer Graphis International (New York, 1993), Springer Verlag, pp. 399-412. 
[236] Vaillant, R., Barthe, L., Guennebaud, G., Cani, M.-P., Rohmer, D., Wyvill, B., Gourmel, O., AND Paulin, M. Implicit skinning: real-time skin deformation with contact modeling. ACM Trans. Graph. 32, 4 (July 2013), 125:1-125:12.

[237] Vaillant, R., Guennebaud, G., Barthe, L., Wyvill, B., AND CANI, M.-P. Robust iso-surface tracking for interactive character skinning. In SIGGRAPH ASIA 2014 (Nov. 2014), vol. 33 of ACM Trans. Graph., pp. $1-11$.

[238] Valentine, T. Face-space models of face recognition. In Computational, Geometric, and Process Perspectives on Facial Cognition: Contexts and Challenges, M. Wenger and J. Townsend, Eds., Scientific Psychology Series. Taylor \& Francis, 2012.

[239] VernON, C. Introduction to Python scripting for Maya artists. chadvernon.com.

[240] Vlasic, D., Brand, M., Pfister, H., AND Popović, J. Face transfer with multilinear models. In ACM Transactions on Graphics (TOG) (New York, NY, USA, 2005), vol. 24, ACM Press, pp. 426-433.

[241] WAHBA, G. Spline Models for Observational Data. SIAM, 1990.

[242] WAND, M., AND Jones, C. Kernel Smoothing. Chapman and Hall/CRC Monographs on Statistics and Applied Probability Series. Chapman \& Hall, 1995.

[243] WANG, J. Geometric Structure of High-Dimensional Data and Dimensionality Reduction. Springer, 2011.

[244] WANG, X. C., AND PHILlips, C. Multi-weight enveloping: Leastsquares approximation techniques for skin animation. In Proceedings of the 2002 ACM SIGGRAPH/Eurographics Symposium on Computer Animation (New York, NY, USA, 2002), SCA '02, ACM, pp. 129-138. 
[245] Weber, O., Sorkine, O., Lipman, Y., And Gotsman, C. Contextaware skeletal shape deformation. Computer Graphics Forum (Proceedings of Eurographics) 26, 3 (2007).

[246] Weise, T., Bouaziz, S., Li, H., AND Pauly, M. Realtime performance-based facial animation. ACM Transactions on Graphics (Proceedings SIGGRAPH 2011) 30, 4 (July 2011).

[247] Weise, T., LI, H., GoOL, L. V., AND PAuly, M. Face/off: Live facial puppetry. In Proceedings of the 2009 ACM SIGGRAPH/Eurographics Symposium on Computer animation (Proc. SCA'09) (ETH Zurich, August 2009), Eurographics Association.

[248] Wendland, H. Scattered Data Approximation. Cambridge, 2005.

[249] Wilhelms, J., AND VAn Gelder, A. Anatomically based modeling. In Proceedings of the 24th Annual Conference on Computer Graphics and Interactive Techniques (New York, NY, USA, 1997), SIGGRAPH '97, ACM Press/Addison-Wesley Publishing Co., pp. 173-180.

[250] Williams, L. Performance-driven facial animation. In SIGGRAPH 90 Conference Proceedings (Aug. 1990), vol. 24, pp. 235-242.

[251] WiLliamS, L. personal communication. Disney Feature Animation, 2001.

[252] Wilson, C. A., Alexander, O., Tunwattanapong, B., Peers, P., Ghosh, A., Busch, J., Hartholt, A., And Debevec, P. Facial cartography: interactive high-resolution scan correspondence. In ACM SIGGRAPH 2011 Talks (New York, NY, USA, 2011), SIGGRAPH '11, ACM, pp. 8:1-8:1.

[253] WiskotT, L., Fellous, J.-M., KRÜGer, N., AND VON DER MAlSBURG, C. Face recognition by elastic bunch graph matching. In Proc. 7th Intern. Conf. on Computer Analysis of Images and Patterns, CAIP'97, 
Kiel (Heidelberg, 1997), G. Sommer, K. Daniilidis, and J. Pauli, Eds., no. 1296, Springer-Verlag, pp. 456-463.

[254] Xia, J., Chandrasekaran, S., Gu, M., And Li, X. S. Fast algorithms for hierarchically semiseparable matrices. Numerical Linear Algebra with Applications 17, 6 (2010), 953-976.

[255] XIA, S., WANG, C., CHAI, J., AND HODGINS, J. Realtime style transfer for unlabeled heterogeneous human motion. ACM Trans. Graph. 34, 4 (July 2015), 119:1-119:10.

[256] Xian, X., Lewis, J., SoON, S. H., Fong, N., AND Tian, F. A Powell optimization approach for example-based skinning in a production animation environment. In Computer Animation and Social Agents (CASA) (2006).

[257] XIAN, X., SOON, S. H., FONG, N., AND TIAN, F. Spherical skinning from examples. In International Workshop on Advanced Image Technology (IWAIT) (2007).

[258] XIAO, J., CHAI, J., AND KANADE, T. A closed-form solution to nonrigid shape and motion recovery. In The 8th European Conference on Computer Vision (ECCV 2004) (May 2004).

[259] YAMANE, K., AND NAKAMURA, Y. Natural motion animation through constraining and deconstraining at will. IEEE Tran. Visualization and Computer Graphics 9, 3 (2003), 352-360.

[260] ZhanG, L., Snavely, N., Curless, B., and Seitz, S. M. Spacetime faces: high resolution capture for modeling and animation. In SIGGRAPH (New York, NY, USA, 2004), ACM, pp. 548-558.

[261] Zhang, Q., Liu, Z., GuO, B., Terzopoulos, D., AND Shum, H.Y. Geometry-driven photorealistic facial expression synthesis. IEEE 
Transactions on Visualization and Computer Graphics 12, 1 (2006), 4860.

[262] ZHAO, X. personal communication. Disney Feature Animation, 2001.

[263] Zhu, X., Ghahramani, Z., AND LAfFerty, J. D. Semi-supervised learning using gaussian fields and harmonic functions. In Machine Learning, Proceedings of the Twentieth International Conference (ICML 2003), August 21-24, 2003, Washington, DC, USA (2003), pp. 912-919.

[264] ZOU, H., AND HASTIE, T. Regularization and variable selection via the elastic net. Journal of the Royal Statistical Society: Series B (Statistical Methodology) 67, 2 (2005), 301-320.

[265] Zurdo, J. S., Brito, J. P., And Otaduy, M. A. Animating wrinkles by example on non-skinned cloth. IEEE Transactions on Visualization and Computer Graphics 19, 1 (2013), 149-158.

[266] ZWERMAn, S., AND OKUn, J. The VES Handbook of Visual Effects: Industry Standard VFX Practices and Procedures. CRC Press, 2014. 


\section{Part IV}

Appendices 


\section{Appendix A}

\section{Mathematical Concepts}

This chapter gives a short tutorial overview of some concepts from machine learning that may be less familiar in graphics, also indicating places where they appear in the thesis. The descriptions are in terms of mathematical language (in particular, linear algebra) that may be familiar to experts in computer graphics.

\section{A.1 Interpolation, Approximation, and Regres- sion}

Many problems can be formulated as interpolation, approximation, or regression. Intuitively speaking, these appear to be variations of the same underlying problem.

- Interpolation is a core technique in computer graphics. Spline interpolation is particularly familiar, as it is frequently used for both keyframe animation and for modeling 3D surfaces. In these applications the domain of interpolation is time and space respectively. In contrast, this thesis will consider applications where the domain is both more abstract and quite high-dimensional, such as the space of facial expressions or of body poses. 
- Regression is less familiar in computer graphics. In contrast to interpolation, regression often assumes that the data is corrupted by noise, and regression methods may provide an estimation of variance as well as the predicted value.

Some interpolation algorithms such as the radial basis function (RBF) approaches surveyed in chapter 8 have variants that do approximation, in which the constructed curve or function trades exact interpolation for an increase in smoothness. Approximation is justified when the data includes noise, and somewhat blurs the distinction between regression and interpolation.

Interpolation and regression are also closely related to machine learning [44]. For example, nearest-neighbor (1-NN) look-up, the simplest learning technique, can be viewed as piecewise constant interpolation. The RBF approaches in chapter 8 were first popularized in the machine learning community [44, 32]. Classification can sometimes be formulated as interpolation of labels, by relaxing the requirement that the labels are binary or discrete [263].

In computer graphics, interpolation also increasingly refers to deformation approaches that approximately locally preserve properties (such as rigidity) of the transformation that maps an undeformed surface or volume to its deformed state [2, 223]. The actual interpolation at an arbitrary point is then accomplished by the polygons or simplices defined by these vertices.

\section{A.2 Parametric versus Non-Parametric}

In statistical terminology a parametric model is one that can be expressed in closed form with a fixed and typically small number of parameters. The Gaussian density, with its mean and variance parameters, is the prototypical example. A non-parametric model is one in which the number of "parameters" potentially grows with the available data. A kernel density 
estimate [242] is a standard example. In the statistical literature splines are also considered as nonparametric.

Adopting this terminology, many early and classic graphics algorithms can justifiably be termed parametric. As mentioned earlier, Parke's pioneering early work on facial animation involved designing a face model with a small number of algorithmically interpreted parameters such as ones that caused a smile or frown to occur. In fact he called his model "parametric" [179]. As another example, the parameters in the linear blend skinning algorithm are the joint transforms and skinning weights.

RBF regression (used in chapters 7f9) is a nonparametric approach, since providing an estimate at a particular point involves all the data. The linear blendshape approaches covered in chapter 3 might not be considered nonparametric, since the examples are used as a basis. Nevertheless, both blendshapes and our use of RBF involve interpolating examples created by the artist, and in both cases the artist can add additional examples to improve the quality of movement. The "example-based" label is appropriate.

\section{A.3 Smoothness, Prior, and Model Complexity}

The related concepts of smoothness, prior, and model complexity have arisen (sometimes independently) in several fields. Given finite data, possibly corrupted with noise, it is usually necessary to assume something about the "true" signal.

In many fields a generic assumption that "signals are smooth" is made. Smoothing splines in statistics reflect this assumption. The smoothness assumption is well suited to animation, where motion tends to be smooth because of physics and because animals have evolved or learned to move in an energy-efficient way [224, 123, Chapter 4: Movement Economy].

A prior is an assumption of the distribution of the data. This is a stronger and more sophisticated assumption. We discuss and use a Gaussian prior 
in chapters 6, 4 , and 5

These assumptions are related to model complexity and the bias/variance trade-off. The textbook example of the latter is the problem of fitting a polynomial to data. A high-order polynomial has low bias but high variance. It can fit the data exactly. however, if the data is corrupted by noise, the high-order polynomial will fit the noise, and will vary as the noise changes. Conversely, a line fit has high bias but low variance. In this case a preference for similar models has a similar effect to the smoothness assumption. Model complexity also interacts with dimensionality: for a fixed level of smoothness, a model's complexity must grow exponentially with dimensionality [59].

In several regression and fitting methods, regularization provides a method to control the bias-variance trade-off. Here is an example for the simple case of the least squares fit $\min _{\mathbf{w}}\|\mathbf{y}-\mathbf{B w}\|^{2}$ where $\mathbf{y}$ is some data to be approximated, $\mathrm{B}$ is a basis, and $\mathrm{w}$ are weights. Solving for the weights,

$$
\mathbf{w}=\left(\mathbf{B}^{T} \mathbf{B}\right)^{-1} \mathbf{B}^{T} \mathbf{y}
$$

and substituting into the prediction equation $\hat{\mathbf{y}}=\mathrm{Bw}$, gives

$$
\hat{\mathbf{y}}=\mathbf{B w}=\mathbf{B}\left(\mathbf{B}^{T} \mathbf{B}\right)^{-1} \mathbf{B}^{T} \mathbf{y}
$$

Looking at the dimensions of $\mathbf{y} \approx \mathbf{B w}$, assume $\mathbf{y}$ is of length $n, \mathbf{w}$ is of size $m<n$, and $\mathbf{B}$ is of size $n \times m$. In this case $\mathbf{y}$ has $n$ degrees of freedom.

However, the matrix $\mathbf{H}=\mathbf{B}\left(\mathbf{B}^{T} \mathbf{B}\right)^{-1} \mathbf{B}^{T}$ is of size $n \times n$, but is of rank $m$. Thus, since $\hat{\mathbf{y}}=\mathbf{H y}, \hat{\mathbf{y}}$ can only have $m$ degrees of freedom, while the error $\mathbf{y}-\hat{\mathbf{y}}$ has $n-m$ degrees of freedom.

The trace of $\mathbf{H}$ is $m$, the number of degrees of freedom:

$$
\begin{aligned}
& \operatorname{tr} \mathbf{B}\left(\mathbf{B}^{T} \mathbf{B}\right)^{-1} \mathbf{B}^{T} \\
& =\operatorname{tr} \mathbf{B}^{T} \mathbf{B}\left(\mathbf{B}^{T} \mathbf{B}\right)^{-1} \\
& =\operatorname{tr} \mathbf{I}_{m}=m
\end{aligned}
$$


Now consider the fit with weight-decay regularization:

$$
\min _{\mathbf{w}}\|\mathbf{y}-\mathbf{B w}\|^{2}+\lambda \mathbf{w}^{T} \mathbf{w}
$$

Solving for the weights and substituting,

$$
\hat{\mathbf{y}}=\mathbf{B}\left(\mathbf{B}^{T} \mathbf{B}+\lambda \mathbf{I}\right)^{-1} \mathbf{B}^{T} \mathbf{y}
$$

A very large $\lambda$ causes $\mathbf{B}^{T} \mathbf{B}+\lambda \mathbf{I} \approx \lambda \mathbf{I}$. The inverse of this is $\lambda^{-1} \mathbf{I}$, which gives a small trace. Thus $\lambda$ affects the trace, and thereby adjusts the effective degrees of freedom or complexity of the model [100].

Perhaps the deepest viewpoint on these issues is provided by algorithmic (Kolmogorov) complexity [143]. Suppose you flip a coin about one million times, and then discover that the resulting sequence is exactly the binary encoding of one of the works of Shakespeare. You would think that something is very fishy! However, this particular sequence of bits is no more or less likely than any other. From a probability viewpoint, there is no reason to think the particular outcome is suspicious.

The resolution of this paradox is the notion of Kolmogorov universal probability. The universal probability of a bitstring $x$ is

$$
P_{U}(x)=\sum_{p: U(p)=x} 2^{-|p|}
$$

where $U(p)$ denotes the result of running the program $p$. In other words, it is the probability that a randomly generated program will generate the string $x$. This is a formalization of Occam's razor, the belief that one should explain phenomena with simple rather than complex causes (or programs). The probability of generating Shakespeare with a short program (or simple cause) is vastly higher than the probability of doing so by chance.

We apply simple weight-decay regularization in chapter 8 . Section 5.5 applies a regularization that penalizes shapes that are not present in the training data. Section 8.2.3 explores whether a function regularization procedure can help detect outliers in artist-provided training data. The kernel 
in RBF interpolation serves as a form of smoothness assumption or prior. In chapter 9 we show that the kernel serves the same role as the covariance in Gaussian process when estimating the process mean.

\section{A.4 Radial basis functions}

Radial basis functions (RBF) are most often understood to refer to the radial kernel used in support vector machines and other kernel methods. In this thesis the term refers to an interpolation, approximation, or regression method in which the predicted quantity is a weighted sum of radial kernels situated at each data point:

$$
f(\mathbf{p})=\sum w_{k} R\left(\left\|\mathbf{p}-\mathbf{p}_{k}\right\|\right)
$$

This RBF regression became briefly popular in the machine learning community in the early 1990s [32]. It was seen as a learning approach with predictable architecture and learning times, since it requires only solving a linear system in the number of training examples. RBF regression is also attractive in that the same algorithm can be trivially adapted to data of any dimensionality. It also allows interpolation of scattered data [137, 9], whereas the interpolation algorithms appearing in graphics textbooks require that data be situated on a regular grid in parameter space.

The character of the interpolation can be controlled to some extent through the choice of the RBF kernel $R$. Some choices of kernels have interpretation as the inverse (Green's function) of a derivative operator, as discussed in chapter 8 . In these cases the result of RBF interpolation is equivalent to a particular type of spline.

RBF is the foundation for the example-based skinning method considered in chapter 7. In chapter 9 we show that RBF regression and discrete Gaussian processes are computationally related. 


\section{A.5 Laplacian splines}

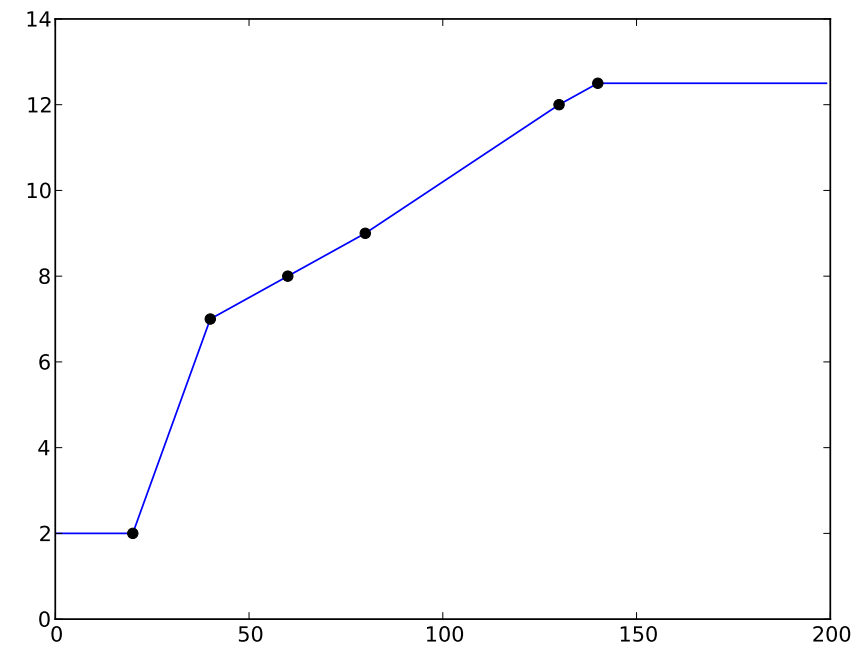

Figure A.1: Laplace interpolation in one dimension is piecewise linear interpolation. Note that this figure was obtained by solving the appropriate linear system (rather than by simply plotting the expected result).

The previous subsection on radial basis methods mentioned choices of kernels for using RBFs to produce thin-plate interpolation. There is an equivalent formulation that does not use RBFs. This formulation minimizes a roughness, expressed as squared derivatives, subject to constraints (boundary conditions). In practice, this results in a single linear system solve for the unknowns via discretization of the Laplacian operator on a mesh. In this mesh-based approach the problem domain is often on a regular grid, so initially this may not seem like scattered interpolation. However, the unknowns can be scattered at arbitrary sites in this grid, so it is effectively a form of scattered interpolation in which the locations are simply quantized to a fine grid. In addition, forms of the Laplacian (Laplace Beltrami) operator have been devised for irregular meshes [97], allowing scattered interpolation on irregular geometry. 
The Laplace equation is obtained by minimizing the integral of the squared first derivative over the domain, with the solution (via the calculus of variations ) that the second derivative is zero:

$$
\min _{f} \int\|\nabla f\|^{2} d \Omega \quad \Rightarrow \quad \nabla^{2} f=0
$$

Similarly, the biharmonic equation is obtained by minimizing the integral of the squared second derivative over the domain, with the solution that the fourth derivative is zero:

$$
\min _{f} \int\left(\left|\frac{\partial^{2} f}{\partial x^{2}}\right|^{2}+2\left|\frac{\partial^{2} f}{\partial x \partial y}\right|^{2}+\left|\frac{\partial^{2} f}{\partial y^{2}}\right|^{2}\right) d x d y \quad \Rightarrow \quad \Delta^{2} f=0
$$

The Laplace equation can be solved with a constrained linear system. Some intuition can be gained by considering the Laplace equation in one dimension with regularly spaced samples. A finite-difference approximation for the second derivative is:

$$
\frac{d^{2} f}{d x^{2}} \approx \frac{1}{h^{2}}(1 \cdot f(x+1)-2 \cdot f(x)+1 \cdot f(x-1))
$$

The weight stencil $(1,-2,1)$ is important. If $f(x)$ is digitized into a vector $f=[f[1], f[2], \cdots, f[m]]$ then the second derivative can be approximated with a matrix expression

$$
\mathbf{L f} \propto\left[\begin{array}{cccccc}
-1 & 1 & & & & \\
1 & -2 & 1 & & & \\
& 1 & -2 & 1 & & \\
& & 1 & -2 & 1 \cdots \\
& & \ldots & &
\end{array}\right]\left[\begin{array}{c}
f[1] \\
f[2] \\
f[3] \\
\vdots
\end{array}\right]
$$

In two dimensions the corresponding finite difference is the familiar Laplacian stencil

$$
\left[\begin{array}{rrr} 
& 1 & \\
1 & -4 & 1 \\
& 1 &
\end{array}\right]
$$


These weights are applied to the pixel sample $f(x, y)$ and its four neighbors $f(x, y-1), f(x-1, y), f(x+1, y), f(x, y+1)$. A two-dimensional array of pixels is in fact regarded as being "vectorized" into a single column vector $f(x, y) \equiv f_{k}$ with $k=y \times$ xres $+x$.

Regardless of dimension the result is a linear system of the form $\mathbf{L f}=0$, with some additional constraints that specify the value of $f\left(x_{k}\right)$ at specific positions. When the constraints are applied this becomes an $\mathbf{L x}=\mathbf{b}$ system rather than a $\mathbf{L x}=\mathbf{0}$ nullspace problem.

A Poisson problem is similar, differing only in that the right hand side of the equation is non-zero. The problem arises by requiring that the gradients of the solution are similar to those of a guide function, where similar is defined as mininimizing the sum (or integrated) squared error. The Laplace equation is just a special case in which the "guide function" is 0 , meaning that the desired gradients should be as small as possible, resulting in a function that is smooth in this sense. In fact, functions derived in this way are sometimes called (Laplacian) splines [57].

In matrix terms, the corresponding thin-plate problem is of the form

$$
\mathbf{L}^{2} \mathbf{f}=\mathbf{b}
$$

where (again) some entries of $f$ are known (i.e. constrained) and are pulled to the right hand side.

In both cases the linear system can be huge, with a matrix size equal to the number of vertices (in the case of manipulating a 3D model) or pixels (in a tone mapping or image inpainting application). On the other hand, the matrix is sparse, so a sparse solver can (and must) be used. The Laplace/Poisson equations are also suitable for solution via multigrid techniques, which have time linear in the number of variables.

Laplacian or Poisson interpolation on regular grids was introduced for image editing in [129, 185], and is now available in commercial tools. [28] used Laplacian and biharmonic interpolation on a mesh to drive a face mask with moving motion capture markers. 
A Poisson problem forms the basis for the spacetime editing approach introduced in chapter 5. RBF kernels can sometimes be considered as a dual approach to Laplacian splines, as described in chapter 8 . Splines and principal component analysis have complementary advantages and disadvantages. Chapter 9 describes this and identifies a relation between the two techniques.

\section{A.6 Manifold Learning and PCA}

From a computer graphics perspective, a manifold can be considered as the generalization of a surface. In the case of a closed two-dimensional surface in a 3D scene, each point on the surface has a small neighborhood that resembles a small portion of the 2D plane. Similarly an $n$-dimensional manifold is an object where each point has a neighborhood that resembles an n-dimensional Euclidean space. The 2D surface exists in a 3D scene, while the $n$-dimensional manifold may exist in an "ambient" space of higher than $n$ dimensions.

In machine learning the manifold assumption asserts that, in many data sets, the data is situated on a "manifold" of lower dimensionality than the (ambient) dimensionality of the data. Manifold learning is the classic machine learning problem of identifying a set of $n$ "latent" variables that parameterize the manifold, starting with only the data. A typical demonstration example involves a set of images of a person's head as they look left to right and up/down. Here the ambient space is the dimensionality of the images (such as $100 \times 100=10000$ ), and the latent space is two dimensions (left-right and up-down). In this case the relationship between the head rotation angles and the image pixels is not linear, and nonlinear manifold learning is required [230, 202].

Principal component analysis (PCA) can be considered as form of manifold learning in which there is a linear relation between the observed data and its lower-dimensional representation. PCA provides a set of orthogo- 


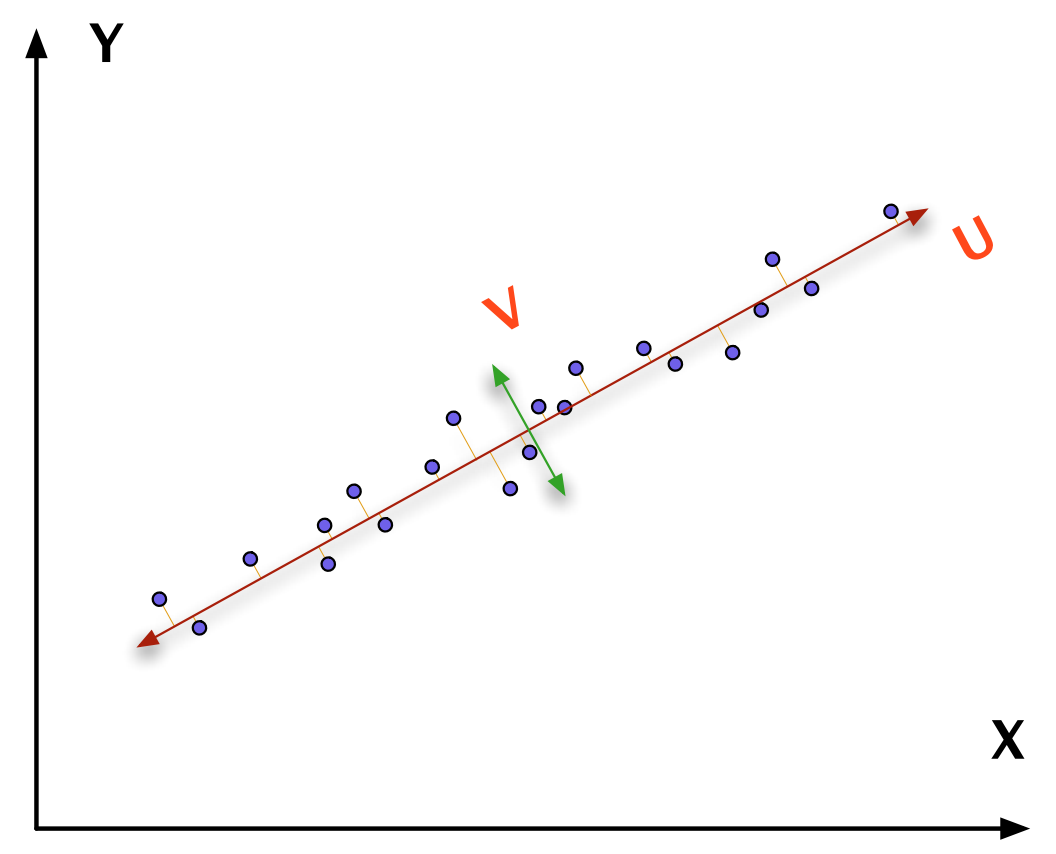

Figure A.2: Motivating illustration for PCA: given a collection of 2D data points that are mostly along a line, PCA finds new axes $U$ and $V$. The axis $U$ is the vector that captures the most variance in the data. The data can be summarized or compressed by retaining only the single number that describes the position of each point along $U$, rather than the two numbers that represent the position of each point in $X$ and $Y$. 
nal axes that describe the data, together with the corresponding variance of the data in the direction of each axis. Figure A.2 shows a standard motivating illustration for PCA. The data in this figure is situated "mostly along a line". The variance along this line $(U)$ is high, whereas the variance along a second axis $(V)$ perpendicular to this line is low. The variation in this second direction can often be ignored. The data can then be compressed by retaining only the single coefficient of each data point with respect to this primary (principal) axis, resulting in a two-to-one data compression.

PCA is widely used. It is also a primary metaphor for thinking about various problems, even when it is not literally employed. In this thesis chapters 3,4 and 9 make use of PCA.

The "axes" in PCA are the eigenvectors of the covariance matrix of the data. The principal axis identified in PCA is the leading eigenvector of the covariance matrix, i.e. the eigenvector corresponding to the largest eigenvalue. This result is obtained from the goal of finding the axis that captures the direction of most variance in the data. To express this mathematically, recall that variance is the expectation of the squared deviation from the mean, $\mathbb{E}\left[(\mathbf{x}-\boldsymbol{\mu})^{2}\right]$. PCA seeks an axis $\mathbf{u}$ such that the variance of the projected data is largest. The projection of a data point $\mathrm{x}$ on a unit-length vector $\mathbf{u}$ is $\left(\mathbf{u}^{T} \mathbf{X}\right) \mathbf{u}$, and the position of the data point along the vector is just $\mathbf{u}^{T} \mathbf{x}$. Combining these, the variance of the projected data is

$$
\mathbb{E}\left[\left(\mathbf{u}^{T}(\mathbf{x}-\boldsymbol{\mu})\right)^{2}\right]
$$

We want to find the vector $\mathbf{u}$ that maximizes this,

$$
\begin{aligned}
& \max _{\mathbf{u}} \mathbb{E}\left[\left(\mathbf{u}^{T}(\mathbf{x}-\boldsymbol{\mu})\right)^{2}\right] \\
& =\max _{\mathbf{u}} \quad \mathbf{u}^{T} \mathbb{E}\left[(\mathbf{x}-\boldsymbol{\mu})(\mathbf{x}-\boldsymbol{\mu})^{T}\right] \mathbf{u} \\
& =\max _{\mathbf{u}} \quad \mathbf{u}^{T} \mathbf{C u}
\end{aligned}
$$


where $\mathbf{C}$ is the covariance of the data, estimated as

$$
\mathbf{C} \approx \frac{1}{N} \sum_{k}\left(\mathbf{x}_{k}-\boldsymbol{\mu}\right)\left(\mathbf{x}_{k}-\boldsymbol{\mu}\right)^{T}
$$

The expression $\mathbf{u}^{T} \mathbf{C u}$ can be made as large as desired by making $\mathbf{u}$ bigger, which tells us nothing. Instead, the expression should be maximized subject to $\mathbf{u}$ being fixed length, such as $\|\mathbf{u}\|=1$. In this case, it is intuitive that $\mathbf{u}$ is an eigenvector of $\mathbf{C}$, since the inner product of $\mathbf{C u}$ with $\mathbf{u}$ will be largest when $\mathbf{C u}$ is parallel to $\mathbf{u}$ (for example if $\mathbf{C u}$ is perpendicular to $\mathbf{u}$, the inner product will be zero).

Because $\mathbf{u}$ is an eigenvector of $\mathbf{C}, \mathbf{C u}=\lambda \mathbf{u}$, so

$$
\mathbf{u}^{T} \mathbf{C u}=\lambda \mathbf{u}^{T} \mathbf{u}=\lambda
$$

because $\mathbf{u}^{T} \mathbf{u}=1$. Recall that the variance of the data along the axis $\mathbf{u}$ is $\mathbb{E}\left[\left(\mathbf{u}^{T}(\mathbf{x}-\boldsymbol{\mu})\right)^{2}\right]=\mathbf{u}^{T} \mathbf{C u}$. Thus $\lambda$ is the variance of the data when projected along direction $\mathbf{u}$.

In addition to the mathematical derivation of PCA, there is an intuition that I find helpful. Write the empirical covariance estimate $\frac{1}{N} \sum_{k}\left(\mathbf{x}_{k}-\right.$ $\boldsymbol{\mu})\left(\mathbf{x}_{k}-\boldsymbol{\mu}\right)^{T}$ as $\frac{1}{N} \mathbf{D D}^{T}$ where $\mathbf{D}$ is a matrix whose columns contain the (vectorized) data points. PCA maximizes the expression

$$
\mathbf{u}^{T} \mathbf{D D}^{T} \mathbf{u}
$$

subject to the length constraint on $\mathbf{u}$. Looking at this, a vector $\mathbf{u}$ will maximize this expression if its inner product with each row of $\mathbf{D}^{T}$ is high. Thus, the first PCA vector is the one which is "most similar to all the data points", in the sense of having the highest inner product.

The remaining axes in PCA are found by the same objective of finding directions that describe maximum variance in the data, but with the constraint that these directions are orthogonal to all previously found directions. The corresponding eigenvalues are variances of the data along these directions. PCA can also be derived by finding a set of axes that successively minimize the squared error between the original data and the data projected on those axes. 
In many graphics and some vision problems the dimensionality of each data "point" is higher than the number of data points, as might be the case with a collection of 100 blendshape models, each with thousands of vertices, or a collection of 200 face images, each having thousands of pixels. In these cases there is a useful trick that makes the computation (cubically) proportional to the number of data items rather than their dimension. Suppose we have 200 face images, each of resolution $1000 \times 1000$. Finding the "eigenfaces" would require getting the eigenvectors of a million ${ }^{2}$ matrix, which impractical.

This trick instead allows working with a small $200 \times 200$ matrix. Stack the data as columns of a million $\times 200$ matrix D. First find eigenvectors of $\mathbf{D}^{T} \mathbf{D}$ :

$$
\mathbf{D}^{T} \mathbf{D} \mathbf{u}=\lambda \mathbf{u}
$$

Premultiply by $\mathbf{D}$ and regroup:

$$
\left(\mathbf{D D}^{T}\right)(\mathbf{D u})=\lambda(\mathbf{D u})
$$

In other words, if $\mathbf{u}$ is an eigenvector of $\mathbf{D}^{T} \mathbf{D}$, then $\mathbf{D u}$ is an eigenvector of $\mathrm{DD}^{T}$.

\section{A.7 The curse of dimensionality}

The curse of dimensionality [210][22, p.95] is a collection of phenomena that, taken together, indicate that our geometric intuitions derived from three-dimensional space can be wrong when applied in high-dimensional spaces. In this thesis we will see that character animation can benefit from being formulated as a high-dimensional problem, so the curse of dimensionality is relevant. Specifically, body animation can be viewed as existing in a space whose dimensionality is the number of joint angles or other parameters describing the body pose (chapter 7), while facial animation can be viewed as a point moving in a space of perhaps 100 dimensions (chapter 3). 


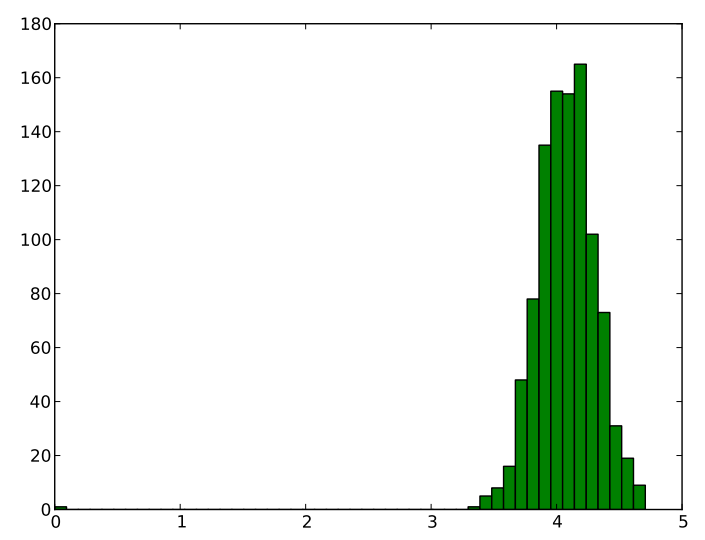

Figure A.3: Histogram of the distances of 1000 100-dimensional pseudorandom variables from the first-generated variable. The pseudorandom variables are uniformly distributed in $0 . .1$ in each dimension. In high dimensions "everything is far away."
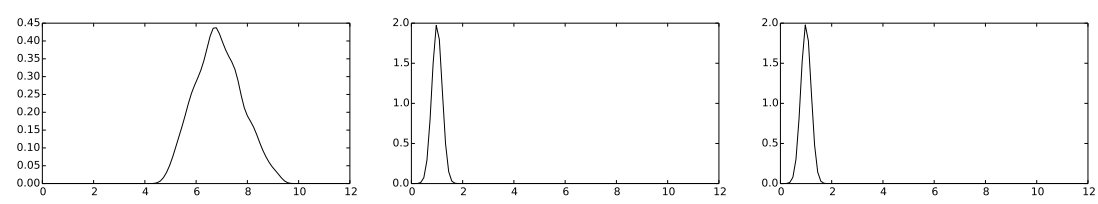

Figure A.4: High-dimensional effect on pairwise distances. Left, kernel density plot of the pairwise Euclidean distances between $30 N(0,1)$ points in 25 dimensions. Middle, histogram of a scaled exponential RBF kernel applied to these distances, $\exp \left(-\left\|\mathbf{p}_{i}-\mathbf{p}_{j}\right\| / 10000\right)$. Right, a scaled Gaussian RBF kernel applied to these distances, $\exp \left(-\left\|\mathbf{p}_{i}-\mathbf{p}_{j}\right\|^{2} / 10000\right)$. In high dimensions, feature maps and kernels discriminate poorly. 


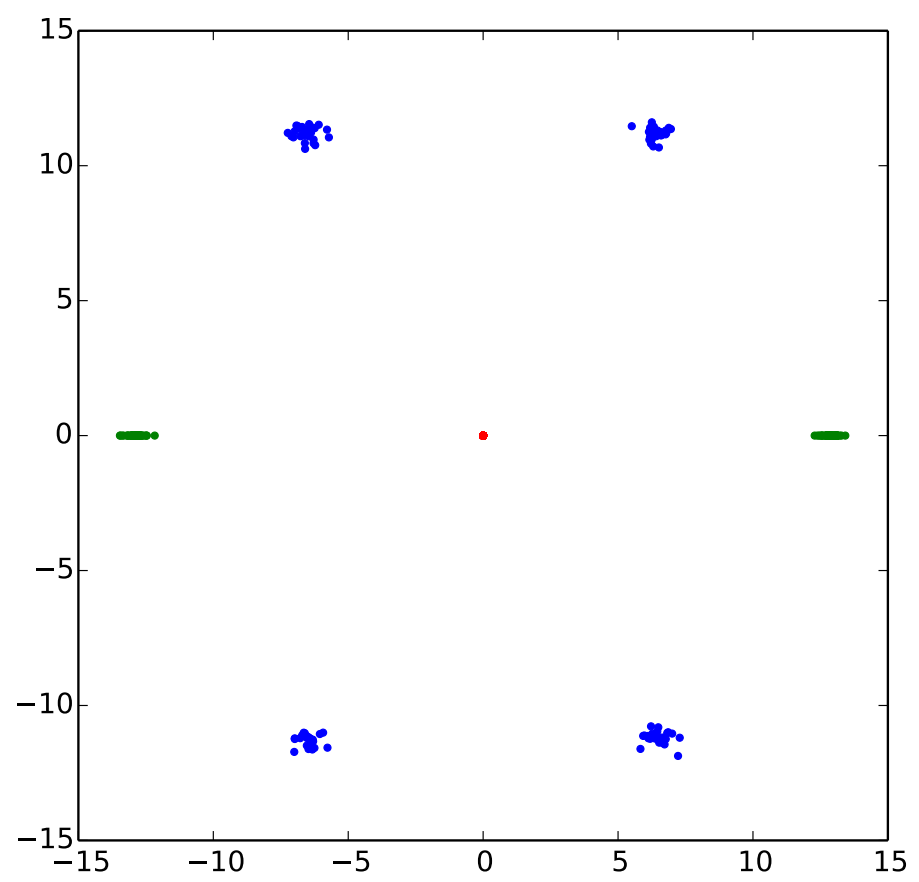

Figure A.5: Points in high dimensions are situated near a randomly rotated lattice. This figure plots 100 triples of randomly chosen points in a 1000 dimensional space. The plane defined by each triple is transformed to align with the $2 \mathrm{D}$ plot with the following procedure: 1) the triple is moved so that the first point is at the origin, 2) the direction from the first point to the second is aligned to the $x=(1,0,0,0,0, \cdots)$ direction using a Householder transformation, 3) lastly, the vector from the first point to the third point is rotated about the $x$ direction to like in the $x, y$ plane. 
The "Curse of dimensionality" takes several forms, including [100, 59]:

1. In $d$ dimensions interpolation and learning methods may need $n^{d}$ times the amount of data.

2. As the number of dimensions increase, every point is approximately equally far away (Figure A.3).

3. In high dimensions, directions to data points are nearly orthogonal (Figure 6.3).

Phenomenon \#1 can be seen intuitively by extrapolating from a set of points on the unit interval in $1 \mathrm{D}$ and in the unit square in 2D. Let the points be uniformly distributed on the $[0,1]$ interval in 1D. Picking any sub-interval of length $1 / 2$, we expect that roughly half of the points will be contained in this interval. Now consider the similar situation on a unit square. The proportion of points contained in a sub-interval of size $1 / 2 \times$ $1 / 2$ will be approximately $1 / 4$ of the total. Similarly, in $n$ dimensions a box of side $1 / 2$ will cover $1 / 2^{n}$ of the points.

This has impact on kernel density computations: in high dimensions, the width of the kernel necessary to cover a specified fraction of points grows to an unusable fraction of the total interval.

This also has an effect on K-nearest-neighbor computations [26, 1]. The percentage of points covered by a kernel of a particular radius is related to the expected distance to the your neighbors. In high dimensions, this expected distance continues to increase even if the total radius is confined to a $n$-cube or ball. Thus the distance between nearest and furthest points, divided by the total distance (to the furthest point) goes to zero. Spatial data structures that put points or distances into bins become increasingly ineffective as the dimension increases.

Another effect is that the distribution of distances between randomly chosen points becomes concentrated in high dimensions. This can be seen as an effect of the the law of large numbers: the distance between two 
points is the square-root of the the sum of squares of the marginal distances. This is the sum of a number of independent random variables, which is concentrated about their expected value. The concentration of distances may affect the kernel-based computations used in the second half of this thesis. Common kernel choices may further compress and homogenize the distribution of distances (Figure A.4).

Perhaps the most remarkable demonstration that our intuitions do not generalize to high dimensions is this: by combining the statements that high-dimensional points are approximately at fixed distances and fixed relative directions, it would seem that these points must lie near a lattice! In fact this is true up to a random rigid transform (Figure A.5.

Another effect of the curse of dimensionality on nonparametric regression can be understood in terms of the bias/variance trade-off. A strategy for reducing bias is to consider local models. However the curse of dimensionality forces all models towards having either global support, or high variance.

The curse of dimensionality arises in chapter 6 when considering the probability of a facial model, and chapter 7 when we consider the highdimensional "pose space" describing the deformation of a human hand.

In regression the curse of dimensionality has led to the use of additive models that have the structure

$$
f\left(x_{1}, \cdots, x_{n}\right) \approx f_{1}\left(x_{1}\right)+\cdots+f_{n}\left(x_{n}\right)
$$

i.e. a sum of "marginal" regressors on each independent variable that ignores cross variable interactions [100]. More generally, as an intuitive statement, there are exponentially (in the dimension) more ways that a function can "wiggle" in high dimensions, and strong limitations on the admissible class of functions are needed if exponential quantities of data are to be avoided.

A promising recent direction for improving high-dimensional regression starts with the manifold assumption (section A.6), i.e. the observation 
that even if the data is high dimensional, it often is situated on a lowerdimensional manifold [243]). If that manifold can be identified (or possibly, even if not [30]) the regression can have the intrinsic manifold dimensionality rather than the larger ambient dimensionality. In chapter 7 we argue that the success of the weighted PSD algorithm introduced by Kurihara and Miyata [120] can be understood in these terms.

\section{A.8 Reproducing Kernel Hilbert Space}

The Reproducing Kernel Hilbert Space (RKHS) idea is an appropriate framework for discussing regression, and is briefly used in several chapters. The RKHS definition requires a number of concepts from functional analysis that are not familiar in graphics. Furthermore, some of this machinery is either not relevant or trivial in a finite computational setting. Nevertheless, some useful aspects of the RKHS idea do apply equally when dealing with discrete, finite data.

Useful aspects of RKHSs are intuitively summarized here:

- A RKHS is a space of well behaved or "smooth" functions that (informally) are generated with a "convolution" of a kernel function $k$ with scattered data,

$$
f(x)=\sum_{1}^{n} \alpha_{i} k\left(x, x_{i}\right)
$$

The smoothness is intuitively evident from the "convolution" in equation (A.2), but can also be understood by a required decay of the spectrum of the kernel, see the discussion of Equation (A.5) below.

- A RKHS provides an alternate formulation of sampling from a function, in which the value of the function is evaluated as a type of inner product with the same kernel that generates the space,

$$
\langle f(x), k(x, y)\rangle_{k}=f(y)
$$


Informally, we would like that sampling of function at a location $x$ should provide information about the function in the neighborhood of $x$. This is not true in the "sifting" formulation of sampling [42] that is familiar in signal processing however. Furthermore, sifting involves the Dirac delta function, which is itself an unusual object that lies outside the space of functions that it is used to sample!

- Putting the previous two properties together,

$$
\langle k(x, y), k(x, z)\rangle_{k}=k(y, z)
$$

i.e. the kernel "reproduces".

- The inner product in Equation A.3 is specific to the RKHS rather than being the usual inner product. It has the form

$$
\langle f, g\rangle_{k}=\sum \sum \alpha_{i} \beta_{j} k\left(x_{i}, x_{j}\right)
$$

Since a norm must be non-negative, it can be seen that the kernel function must be at least positive semidefinite. A second expression for the inner product is

$$
\left\langle\sum_{i}^{\infty} c_{i} u_{i}(x), \quad \sum_{i}^{\infty} d_{i} u_{i}(x)\right\rangle_{k}=\sum \frac{c_{i} d_{i}}{\lambda_{i}}
$$

where $c_{i}, d_{i}$ are coefficients of the expansion of each function in terms of the eigenvectors $u_{i}$ of the kernel, i.e. $f(x)=\sum_{1}^{\infty} c_{i} u_{i}(x)$. Since this corresponds to a squared norm,

$$
\|f\|_{k}^{2}=\sum_{i}^{\infty} \frac{c_{i}^{2}}{\lambda_{i}}
$$

we can see that the coefficients must go to zero sufficiently quickly in order that the norm be finite.

- The representer theorem says that when a problem of fitting a function to data is formulated in an RKHS, in many cases the solution can 
be represented as weighted sum of the kernel centered at each data point, i.e. Equation (A.2). More specifically, for a fitting problem

$$
\min _{f} c\left(x_{i}, y_{i}, f\left(x_{i}\right)\right)+\lambda R\left(\|f\|_{k}\right)
$$

where $c()$ is an arbitrary function measuring the fit of the function $f\left(x_{i}\right)$ to the data $y_{i}$ and $R$ is an arbitrary monotonic function of the RKHS norm of the function $f$, the solution can always be written in the form (A.2) [209].

- RKHSs are connected to the kernel trick in machine learning, in which a linear algorithm can be converted into an algorithm with nonlinear power by nonlinearly mapping the data into a feature space before applying the linear algorithm (sometimes in a dual form). The kernel can also be viewed as mapping a point $x$ to a function, and the kernel specifies the similarity between functions since it specifies an inner product.

RKHS formulations are used in chapters 4 and 9 of this thesis, and in the author's publications [132, 214, 11]. 


\section{Appendix B}

\section{Example derivation of a RBF kernel}

Computer graphics and vision literature present RBF kernels without derivation, and the author had wondered where expressions such as $r^{2} \log r$ "come from".

There are several answers to this. Many radial functions can be used as RBF kernels to exactly interpolate data (conditions are given in [85]), however this gives no guidance on choosing a particular kernel. In fact some commonly used RBF kernels are the Green's functions of differential operators that are used in formulating spline-like interpolation. This interpretation is used in a number of places in this thesis.

In this appendix we derive a RBF kernel for a one-dimensional problem using variational calculus. The goal is to provide an example of what is involved.

The general strategy is to use calculus of variations to setup a minimum for the energy, then use integration by parts to remove the derivatives from the variation test function $\epsilon h(x)$, express as $\int \epsilon h(x)(\cdots) d x$ and note that the $\cdots$ part must be zero. This converts the energy integral into a differential equation. Then we use a Green's function method: take the Fourier transform, solve for the function using simple algebra, take the 
inverse Fourier transform.

The following "energy" defines a thin plate with "tension":

$$
F(f)=\sum\left(f\left(x_{i}\right)-y_{i}\right)^{2}+\alpha \int\|\nabla f\|^{2} d x+\gamma \int\left\|\nabla^{2} f\right\|^{2} d x
$$

The summation covers the available data points, and the integration is over the domain of the spline.

An analogous one-dimensional problem is

$$
F(f)=\sum\left(f\left(x_{i}\right)-y_{i}\right)^{2}+\alpha \int\left\|\frac{d f}{d x}\right\|^{2} d x+\gamma \int\left\|\frac{d^{2} f}{d x^{2}}\right\|^{2} d x
$$

Renotate this as

$$
F(f)=\sum\left(f\left(x_{i}\right)-y_{i}\right)^{2}+\alpha \int\left\|f_{x}\right\|^{2} d x+\gamma \int\left\|f_{x x}\right\|^{2} d x
$$

The minimum of $F(f)$ can be found with variational calculus. In this approach, each occurrence of $f_{x}$ in $F(f)$ is replaced with the variation $f_{x}+$ $\epsilon h_{x}$. At a minimum, the variation

$$
\frac{F(f+\epsilon h)-F(f)}{\epsilon}
$$

will be zero. To reduce notation we will temporarily call $f\left(x_{i}\right), h\left(x_{i}\right), y_{i}$ as $f, h, y$.

For the terms in the data-fit sum $\sum\left(f\left(x_{i}\right)-y_{i}\right)^{2}$,

$$
\begin{aligned}
& (f+\epsilon h-y)^{2} \\
& =f^{2}+e^{2} h^{2}+y^{2}+2 f \epsilon h-2 f y-2 \epsilon h y \\
& =f^{2}-2 f y+y^{2}+2 f \epsilon h-2 \epsilon h y+\epsilon^{2} h^{2} \\
& =(f-y)^{2}+2 \epsilon h(f-y)+\epsilon^{2} h^{2}
\end{aligned}
$$


The first derivative term $\alpha \int\left\|f_{x}\right\|^{2}$ expands to

$$
\begin{aligned}
& \alpha \int\left\|(f+\epsilon h)_{x}\right\|^{2} d x \\
& =\alpha \int(f+\epsilon h)_{x} \quad(f+\epsilon h)_{x} d x \\
& =\alpha \int\left(f_{x}+\epsilon h_{x}\right)\left(f_{x}+\epsilon h_{x}\right) d x \\
& =\alpha \int\left\|f_{x}\right\|^{2}+2 \epsilon f_{x} h_{x}+\epsilon^{2}\left\|h_{x}\right\|^{2} d x
\end{aligned}
$$

The second derivative term $\gamma \int\left\|f_{x x}\right\|^{2} d x$ similarly expands to

$$
\begin{aligned}
& \gamma \int\left\|(f+\epsilon h)_{x x}\right\|^{2} d x \\
& =\gamma \int(f+\epsilon h)_{x x} \quad(f+\epsilon h)_{x x} d x \\
& =\gamma \int\left(f_{x x}+\epsilon h_{x x}\right)\left(f_{x x}+\epsilon h_{x x}\right) d x \\
& =\gamma \int\left\|f_{x x}\right\|^{2}+2 \epsilon f_{x x} h_{x x}+\epsilon^{2}\left\|h_{x x}\right\|^{2} d x
\end{aligned}
$$

Now take the limit of

$$
\frac{F(f+\epsilon h)-F(f)}{\epsilon}
$$

as $\epsilon \rightarrow 0$. In dividing by $\epsilon$, the single $\epsilon$ factors drop, whereas the $\epsilon^{2}$ factors become $\epsilon$. Then taking $\epsilon \rightarrow 0$ the $\epsilon$ becomes zero, effectively removing the $\epsilon^{2}$ terms. In the following we will simply remove these terms as they 
appear.

$$
\begin{aligned}
& F(f+\epsilon h)-F(f) \backslash\left\{\epsilon^{2} \text { terms }\right\}= \\
& \sum\left(f\left(x_{i}\right)-y_{i}\right)^{2}+2 \epsilon h\left(x_{i}\right)\left(f\left(x_{i}\right)-y_{i}\right) \\
& \quad+\alpha \int\left\|f_{x}\right\|^{2}+2 \epsilon f_{x} h_{x} d x \\
& \quad+\gamma \int\left\|f_{x x}\right\|^{2}+2 \epsilon f_{x x} h_{x x} d x \\
& -\quad \sum\left(f\left(x_{i}\right)-y_{i}\right)^{2}-\alpha \int\left\|f_{x}\right\|^{2} d x-\gamma \int\left\|f_{x x}\right\|^{2} d x \\
& =2 \epsilon \sum h\left(x_{i}\right)\left(f\left(x_{i}\right)-y_{i}\right)+2 \epsilon \alpha \int f_{x} h_{x} d x+2 \epsilon \gamma \int f_{x x} h_{x x} d x
\end{aligned}
$$

Pull the sum under an integral by expressing it with a Dirac:

$=2 \int \epsilon \sum h(x)\left(f\left(x_{i}\right)-y_{i}\right) \delta\left(x-x_{i}\right) d x+2 \epsilon \alpha \int f_{x} h_{x} d x+2 \epsilon \gamma \int f_{x x} h_{x x} d x$

Now do two integration by parts (IBP) on the second derivative term, thereby removing the derivative from $h$ and increasing it on $f$. Likewise do one IBP on the first derivative term. Recall the integration by parts pattern:

$$
\int A B_{x} d x=\left.A B\right|_{b} ^{e}-\int B A_{x} d x
$$

where $A_{x}$ is the derivative of some function $A(x)$, and $b, e$ denote the beginning and end of the domain of integration.

The result of applying IBP to the first derivative term is:

$$
2 \epsilon \alpha \int f_{x} h_{x} d x=-2 \epsilon \alpha \int h(x) f_{x x}(x) d x
$$

The $A B$ term in the IBP is zero because the test function $h(x)$ is zero at the boundaries. 
Second derivative IBP

$$
\begin{gathered}
2 \int \epsilon f_{x x} h_{x x} d x \\
\text { first application of IBP: }=-2 \epsilon \gamma \int f_{x x x} h_{x} d x \\
\text { second application of IBP: }=2 \epsilon \gamma \int f_{x x x x} h d x
\end{gathered}
$$

Substituting these IBP expressions, the new $F(f+\epsilon h)-F(f)$ is

$$
\begin{aligned}
& 2 \epsilon \int \sum h(x)\left(f\left(x_{i}\right)-y_{i}\right) \delta\left(x-x_{i}\right) d x-2 \epsilon \alpha \int h(x) f_{x x}(x) d x+2 \epsilon \gamma \int f_{x x x x} h d x \\
& =2 \int \epsilon h(x)\left(\sum\left(f\left(x_{i}\right)-y_{i}\right) \delta\left(x-x_{i}\right) d x-\alpha f_{x x}(x)+\gamma f_{x x x x}\right) d x
\end{aligned}
$$

and finally taking $\frac{F(f+\epsilon h)-F(f)}{\epsilon}$ eliminates the $\epsilon$. This expression is zero at the minimum because it is the variation. Then, because $h(x)$ can be arbitrary, we know that the quantity in parenthesis is zero everywhere:

$$
\gamma f_{x x x x}(x)-\alpha f_{x x}(x)+\sum\left(f\left(x_{i}\right)-y_{i}\right) \delta\left(x-x_{i}\right)=0
$$

(Note that the minus sign came from the IBP).

This differential equation can be solved with the Green's function approach. For a Green's function setup,

$$
\gamma g_{x x x x}(x)-\alpha g_{x x}(x)=\delta(x)
$$

and the function is expressed as a weighted convolution of the Green's function with the data:

$$
f(x)=\sum_{k} w_{k} g\left(x-x_{k}\right)
$$


Substitute this definition into Equation (B.1) (repeated here):

$$
\begin{aligned}
& \gamma f_{x x x x}(x)-\alpha f_{x x}(x)+\sum_{i}\left(f\left(x_{i}\right)-y_{i}\right) \delta\left(x-x_{i}\right)=0 \\
= & \gamma \frac{d^{4}}{d x^{4}}\left(\sum_{k} w_{k} g\left(x-x_{k}\right)\right)-\alpha \frac{d^{2}}{d x^{2}}\left(\sum_{k} w_{k} g\left(x-x_{k}\right)\right)+\sum_{i}\left(f\left(x_{i}\right)-y_{i}\right) \delta\left(x-x_{i}\right)=0 \\
= & \gamma \sum w_{k} \frac{d^{4}}{d x^{4}} g\left(x-x_{k}\right)-\alpha \sum w_{k} \frac{d^{2}}{d x^{2}} g\left(x-x_{k}\right)+\sum_{i}\left(f\left(x_{i}\right)-y_{i}\right) \delta\left(x-x_{i}\right)=0
\end{aligned}
$$

(Changing notation)

$$
\begin{aligned}
& =\gamma \sum w_{k} g_{x x x x}\left(x-x_{k}\right)-\alpha \sum w_{k} g_{x x}\left(x-x_{k}\right)+\sum_{i}\left(f\left(x_{i}\right)-y_{i}\right) \delta\left(x-x_{i}\right)=0 \\
& =\sum w_{k} \gamma g_{x x x x}\left(x-x_{k}\right)-\sum w_{k} \alpha g_{x x}\left(x-x_{k}\right)+\sum_{i}\left(f\left(x_{i}\right)-y_{i}\right) \delta\left(x-x_{i}\right)=0 \\
& =\sum w_{k}\left(\gamma g_{x x x x}\left(x-x_{k}\right)-\alpha g_{x x}\left(x-x_{k}\right)\right)+\sum_{i}\left(f\left(x_{i}\right)-y_{i}\right) \delta\left(x-x_{i}\right)=0 \\
& =\sum w_{k} \delta\left(x-x_{k}\right)+\sum_{i}\left(f\left(x_{i}\right)-y_{i}\right) \delta\left(x-x_{i}\right)=0
\end{aligned}
$$

Where the last step comes from applying Equation (B.2).

Now substitute the expression Equation (B.3) for $f(x)$ in terms of the Green's function on the right hand side, giving

$$
\sum w_{i} \delta\left(x-x_{i}\right)+\sum_{i}\left(\left(\sum_{k} w_{k} g\left(x_{i}-x_{k}\right)\right)-y_{i}\right) \delta\left(x-x_{i}\right)=0
$$

Note the switch from $k$ to $i$ in the leftmost term. This is ok - the $x_{k}$ on the left indexes the same data points as $x_{i}$ on the right.

The $\delta\left(x-x_{i}\right)$ are independent, that is,

$$
\sum a_{k} \delta\left(x-x_{k}\right)=0 \quad \Rightarrow \quad a_{k}=0
$$

so this means

$$
w_{i}+\left(\left(\sum_{k} w_{k} g\left(x_{i}-x_{k}\right)\right)-y_{i}\right)=0 \quad \forall i
$$


or

$$
\sum_{k} w_{k} g\left(x_{i}-x_{k}\right)+w_{i}-y_{i}=0 \quad \forall i
$$

which resembles is a linear system for the approximation problem... but the function $g$ is still unknown! What happened to $\alpha, \gamma$ ? They are "baked" into the Greens function.

To solve for the Greens function $g$ : Go back to the Greens function relation (B.2) and take the Fourier transform,

$$
\begin{gathered}
\gamma j^{4} \omega^{4} G(\omega)-\alpha j^{2} \omega^{2} G(\omega)=1 \\
G(\omega)=\frac{1}{\gamma j^{4} \omega^{4}-\alpha j^{2} \omega^{2}}
\end{gathered}
$$

Since $j^{2}=-1$ and $j^{4}=1$,

$$
G(\omega)=\frac{1}{\gamma \omega^{4}+\alpha \omega^{2}}
$$

To simplify, we will take $\gamma=1$. Then this is equal to

$$
\frac{1}{\omega^{2}} \frac{1}{\alpha+\omega^{2}}
$$

The $\frac{1}{\alpha+\omega^{2}}$ is the transform of the Laplace kernel, so the desired kernel is (any) second antiderivative of the Laplace kernel.

Note that since the original roughness penalty does not "see" constants, a polynomial correction of order 0 (i.e. a constant) is necessary. 